\title{
Immune System Based Control and Intelligent Agent Design for Power System Applications
}

\author{
Rabie Belkacemi \\ West Virginia University
}

Follow this and additional works at: https://researchrepository.wvu.edu/etd

\section{Recommended Citation}

Belkacemi, Rabie, "Immune System Based Control and Intelligent Agent Design for Power System Applications" (2011). Graduate Theses, Dissertations, and Problem Reports. 4693.

https://researchrepository.wvu.edu/etd/4693

This Dissertation is protected by copyright and/or related rights. It has been brought to you by the The Research Repository @ WVU with permission from the rights-holder(s). You are free to use this Dissertation in any way that is permitted by the copyright and related rights legislation that applies to your use. For other uses you must obtain permission from the rights-holder(s) directly, unless additional rights are indicated by a Creative Commons license in the record and/ or on the work itself. This Dissertation has been accepted for inclusion in WVU Graduate Theses, Dissertations, and Problem Reports collection by an authorized administrator of The Research Repository @ WVU.

For more information, please contact researchrepository@mail.wvu.edu. 


\title{
Immune System Based Control and Intelligent Agent Design for Power System Applications
}

\author{
by \\ Rabie Belkacemi \\ Dissertation submitted to the \\ College of Engineering and Mineral Resources \\ at West Virginia University \\ in partial fulfillment of the requirements \\ for the degree of \\ Doctor of Philosophy \\ in \\ Electrical Engineering
}

Prof. Muhammad A. Choudhry, Ph.D.

Prof. Hong-Jian Lai, Ph.D.

Prof. Afzel Noore, Ph.D.

Prof. Daryl Reynolds, Ph.D.

Prof. Ali Feliachi, Ph.D., Chair

Lane Department of Computer Science and Electrical Engineering

\author{
Morgantown, West Virginia
}

2011

Keywords: Control Design, NERC, AGC, Immune System, Multi-Agent System, Fault Detection, Power System Reconfiguration, Hardware, Power Simulator Hardware, DGs, JADE, Communication Hardware.

Copyright 2011 Rabie Belkacemi 


\title{
ABSTRACT
}

\section{Immune System Based Control and Intelligent-Agent Design for Power System Applications}

by

\author{
Rabie Belkacemi
}

The National Academy of Engineering has selected the US Electric Power Grid as the supreme engineering achievement of the 20th century. Yet, this same grid is struggling to keep up with the increasing demand for electricity, its quality and cost. A growing recognition of the need to modernize the grid to meet future challenges has found articulation in the vision of a Smart Grid in using new control strategies that are intelligent, distributed, and adaptive. The objective of this work is to develop smart control systems inspired from the biological Human Immune System to better manage the power grid at the both generation and distribution levels. The work is divided into three main sections. In the first section, we addressed the problem of Automatic Generation Control design. The Clonal Selection theory is successfully applied as an optimization technique to obtain decentralized control gains that minimize a performance index based on Area Control Errors. Then the Immune Network theory is used to design adaptive controllers in order to diminish the excess maneuvering of the units and help the control areas comply with the North American Electric Reliability Corporation's standards set to insure good quality of service and equitable mutual assistance by the interconnected energy balancing areas. The second section of this work addresses the design and deployment of Multi Agent Systems on both terrestrial and shipboard power systems self-healing using a novel approach based on the Immune Multi-Agent System (IMAS). The Immune System is viewed as a highly organized and distributed Multi-Cell System that strives to heal the body by working together and communicating to get rid of the pathogens. In this work both simulation and hardware design and deployment of the MAS are addressed. The third section of this work consists in developing a small scale smart circuit by modifying and upgrading the existing Analog Power Simulator to demonstrate the effectiveness of the developed technologies. We showed how to develop smart Agents hardware along with a wireless communication platform and the electronic switches. After putting together the different designed pieces, the resulting Multi Agent System is integrated into the Power Simulator Hardware. The multi Agent System developed is tested for fault isolation, reconfiguration, and restoration problems by simulating a permanent three phase fault on one of the feeder lines. The experimental results show that the Multi Agent System hardware developed performed effectively and in a timely manner which confirms that this technology is very promising and a very good candidate for Smart Grid control applications. 


\section{ACKNOWLEDGMENT}

I would like to thank my advisor, Dr. Ali Feliachi, for his training, guidance and encouragement throughout my studies and research work at West Virginia University. I would also like to express my gratitude to Dr. Muhammad Choudhry for his direction and help during my research work. I am also thankful to Dr. Afzel Noore, Dr. Daryl Reynolds and Dr. H.J. Lai for their guidance and feedback. I also express my thanks to all the faculty members and students of APERC for making my time enjoyable. I would like to thank the CSEE Department and the University for allowing me to use the facilities and equipment. Lastly, I am very thankful to my parents and family and especially my wonderful wife for her love, encouragement and support. 


\section{CONTENTS}

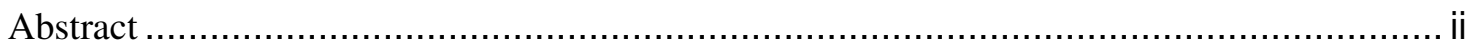

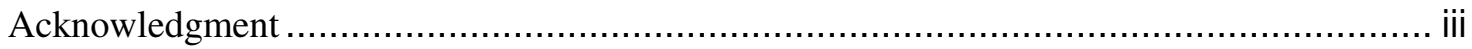

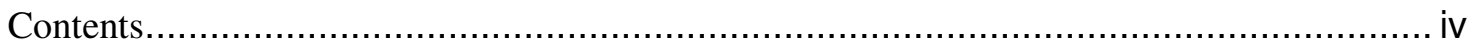

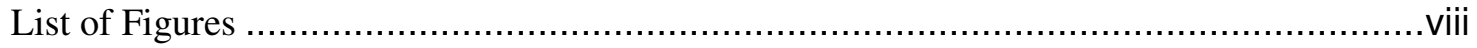

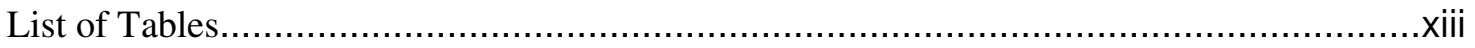

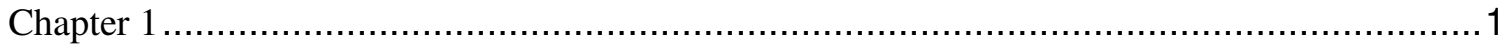

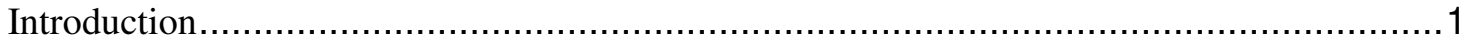

1.1 Decentralized load frequency control design problem ........................................

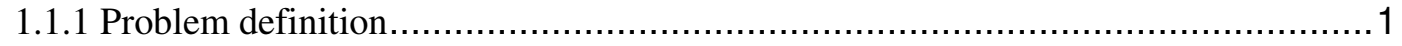

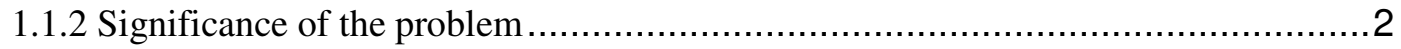

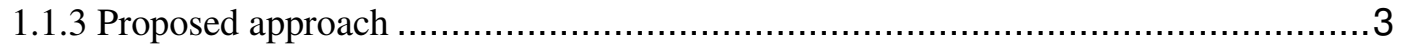

1.2 Electric distribution system self-healing problem ……................................... 4

1.2.1 Problem definition ..............................................................................

1.2.2 Significance of the problem .....................................................................

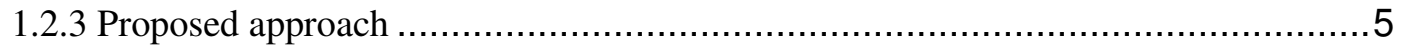

$1.3 \quad$ Dissertation Outline

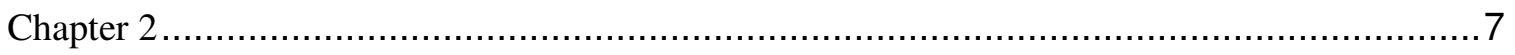

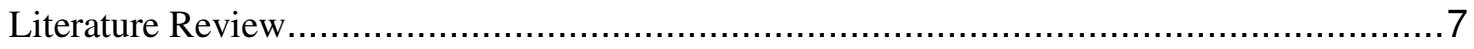

2.1 Power System Load Frequency Control Design ……..........................................

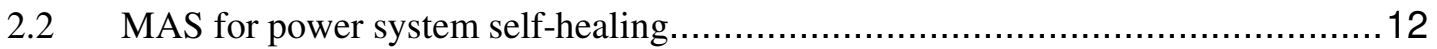

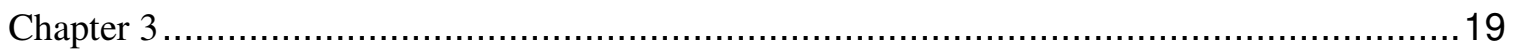


A Decentralized Immune Based Control Design of an Automatic Generation Control in Compliance with the NERC's Standards ........................................................ 19

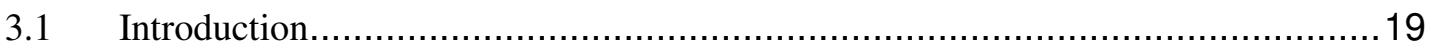

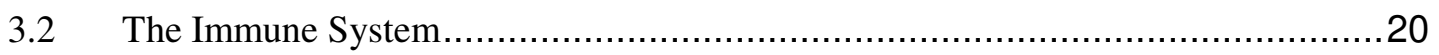

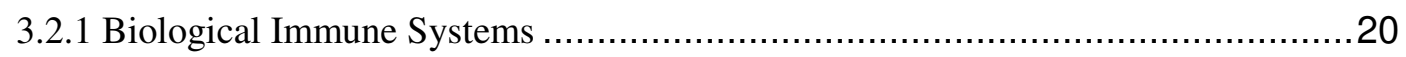

3.2.2 The Antibody factory: the B Cells ..................................................... 23

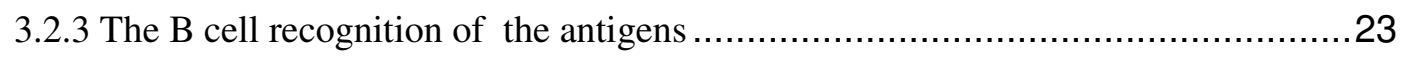

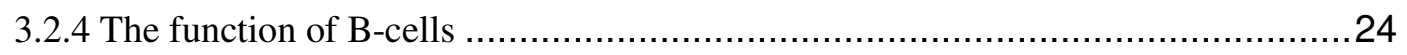

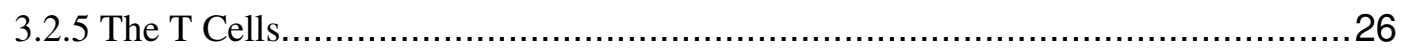

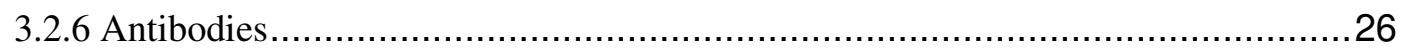

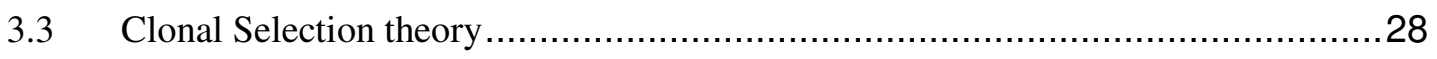

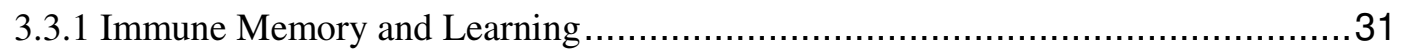

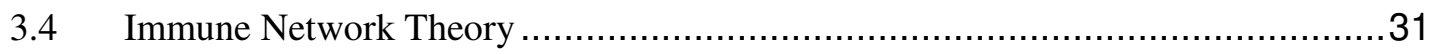

3.5 Computational Aspects of the Immune System .......................................... 32

3.6 Power System - Human Body Analogy ............................................... 33

3.7 Automatic Generation control (AGC) problem ….................................... 35

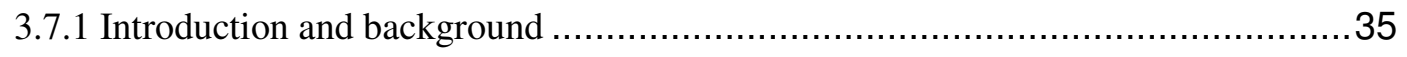

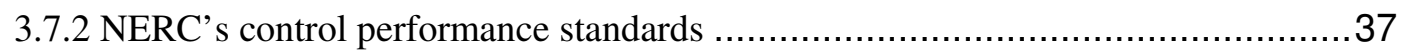

3.8 Immune based optimization using clonal selection theory .............................. 38

3.9 Immune based NERC standards oriented controller design ............................40

3.9.1 Designing the Immune System Network ............................................ 41

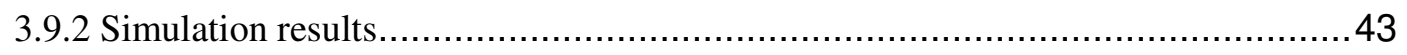

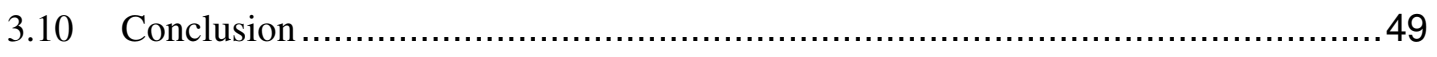

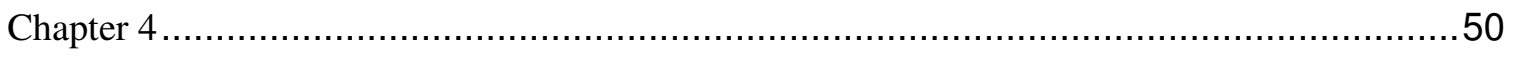

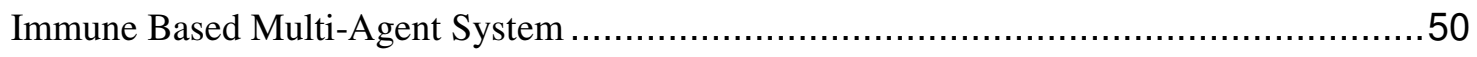




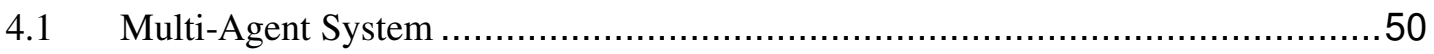

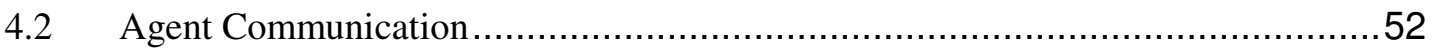

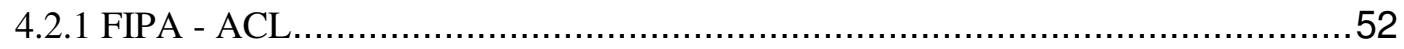

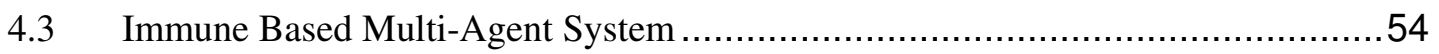

4.4 Implementation on SCE's Circuit of the future Model .................................57

4.5 Hardware Prototype: A shipboard power system ....................................... 67

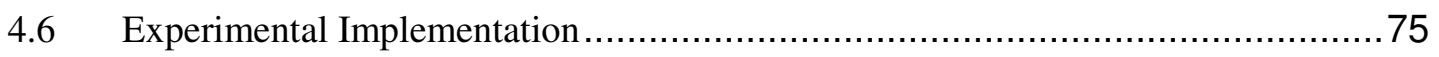

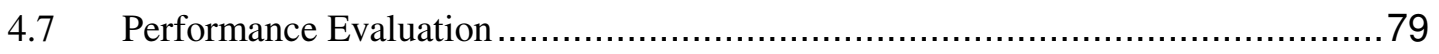

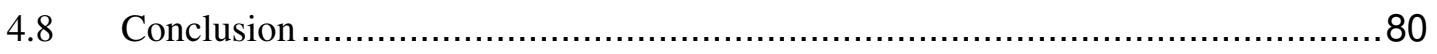

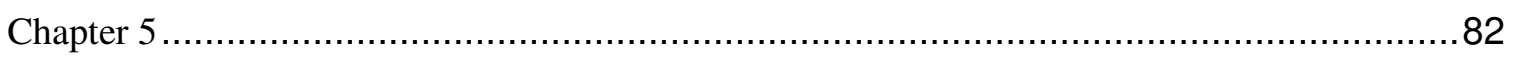

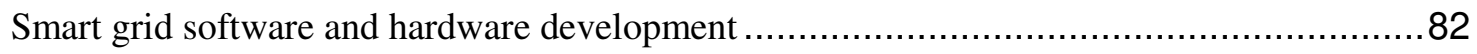

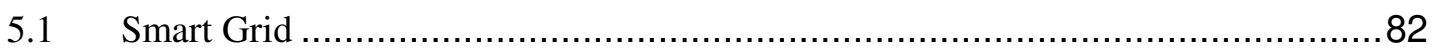

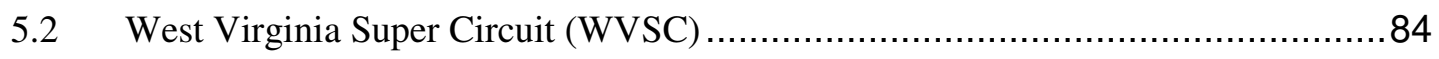

5.2.1 Fault Location Isolation and Restoration .......................................... 84

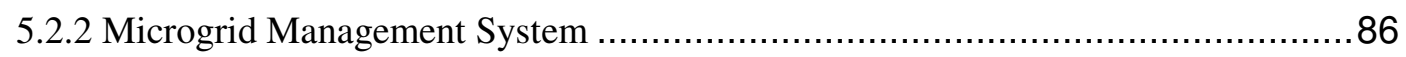

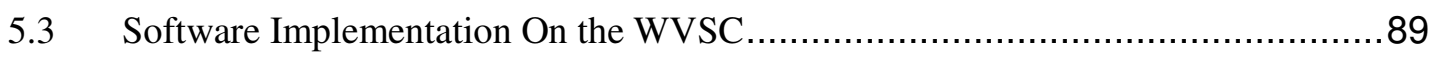

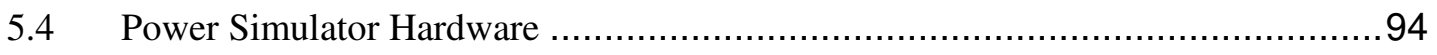

5.5 Hardware Agent Design and integration.................................................. 97

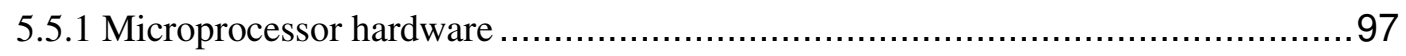

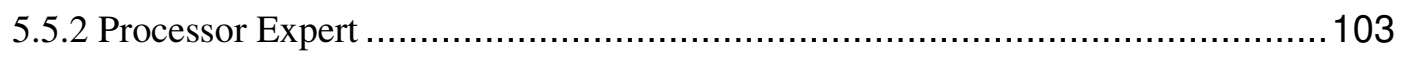

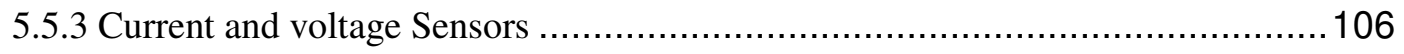

5.5.4 Hardware Communication platform ............................................... 110

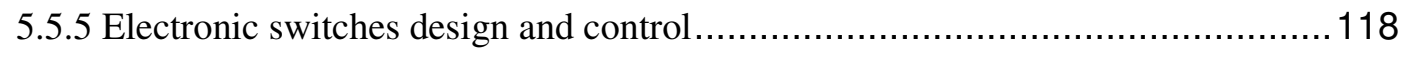

5.5.6 Integration and Testing on the Power Simulator .................................... 121

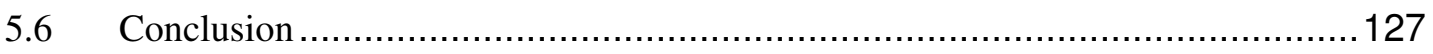




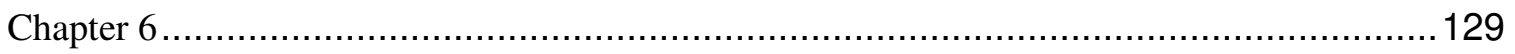

Conclusion and Future work ...................................................................... 129

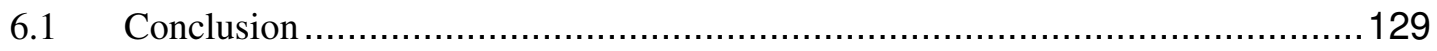

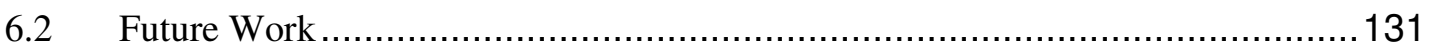

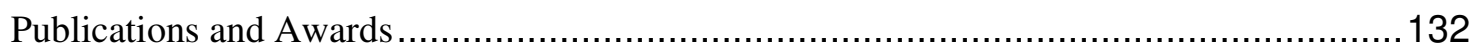

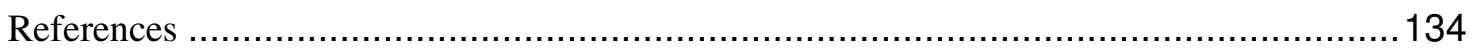




\section{LIST OF FIGURES}

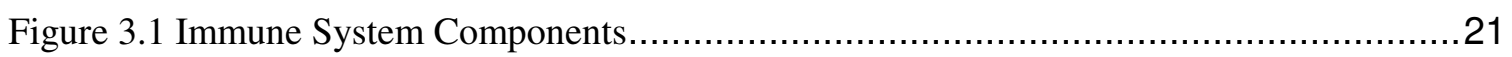

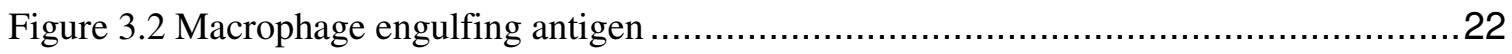

Figure 3.3 Stimulated B-cell acting on the power system ....................................... 25

Figure 3.4 Example of gene combination in power systems........................................27

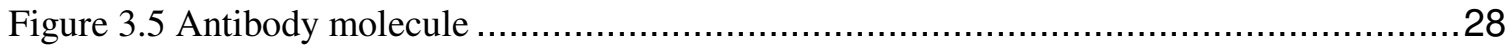

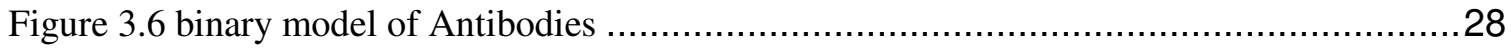

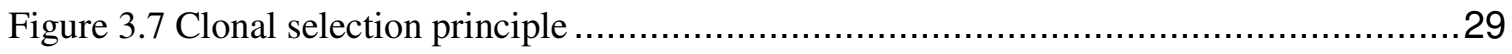

Figure 3.8 Mutation process of the antibodies....................................................... 30

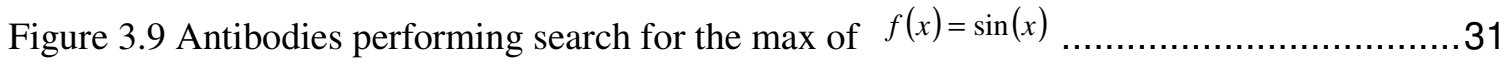

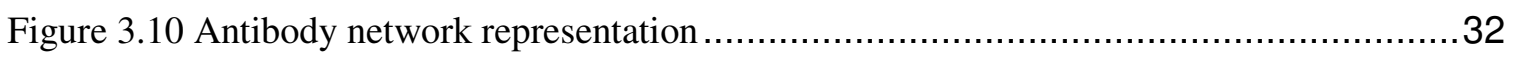

Figure 3.11 Human body-power systems analogy ............................................... 34

Figure 3.12 Two area system model with AGC loop ….......................................... 36

Figure 3.13 Distribution of the antibodies after the $1^{\text {st }}$ generation ..................................... 39

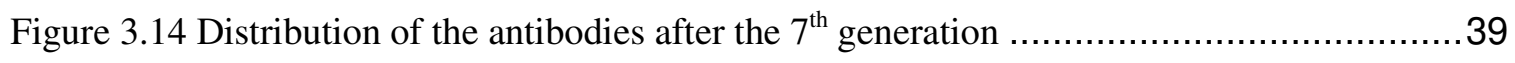

Figure 3.15 Distribution of the antibodies after the $11^{\text {th }}$ generation ................................40

Figure 3.16 The proposed decentralized control................................................ 40

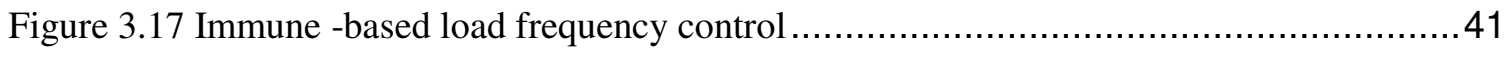

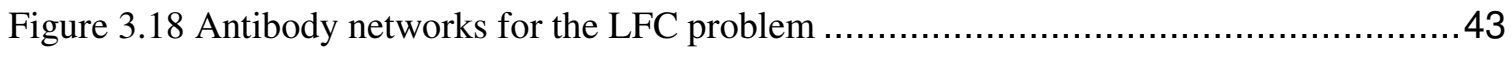

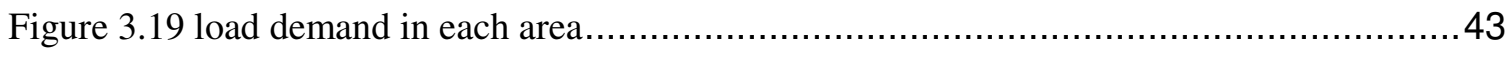

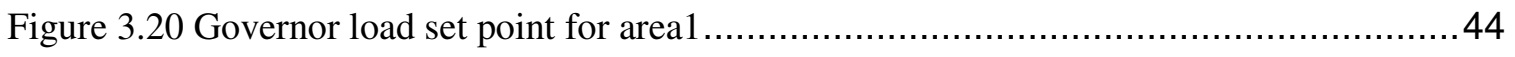

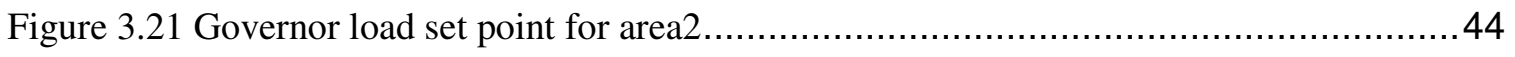

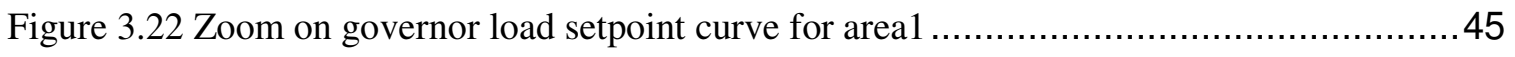




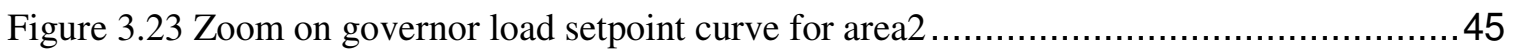

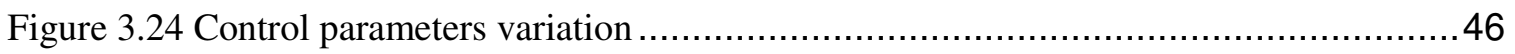

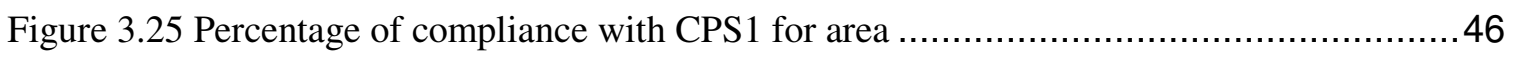

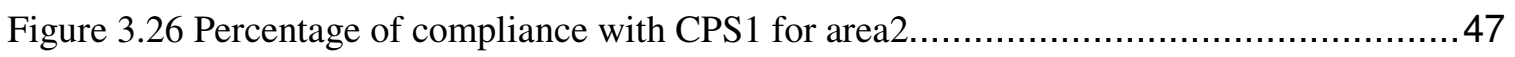

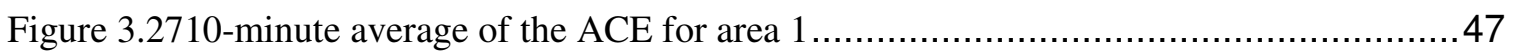

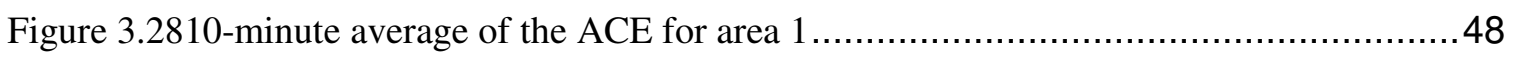

Figure 4.1 Multi-agent System in the sense of Power System ..................................... 51

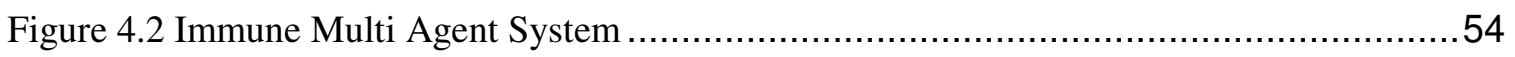

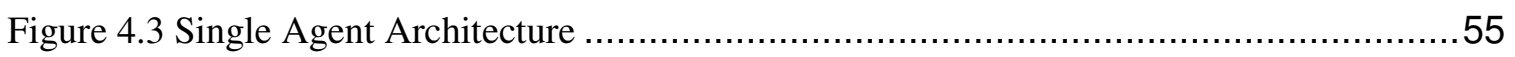

Figure 4.4 Southern California Edison Distribution System.......................................58

Figure 4.5 Agent communication following a fault in the system ..................................59

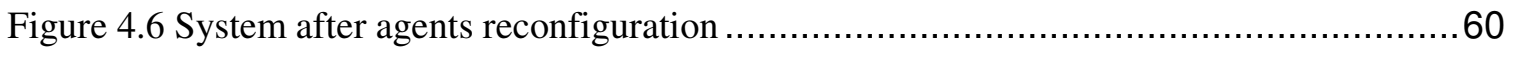

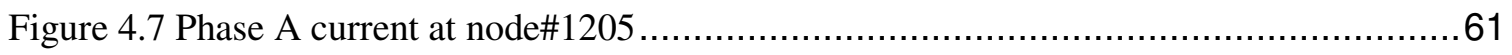

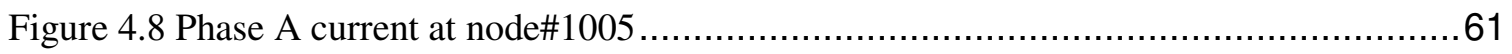

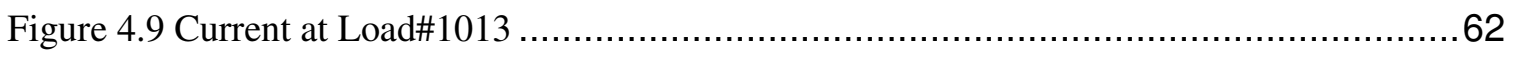

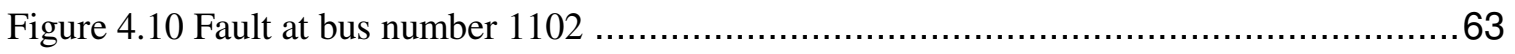

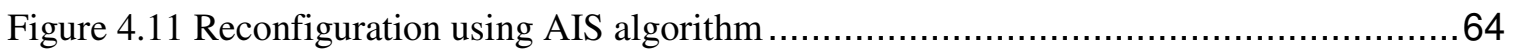

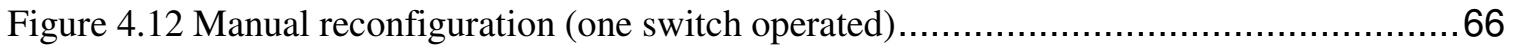

Figure 4.13 Manual reconfiguration (two switches operated) ................................... 67

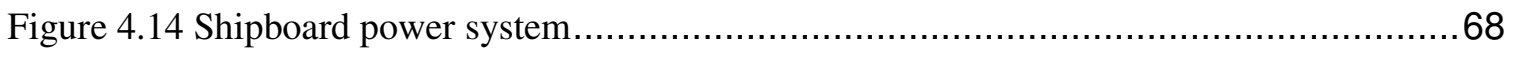

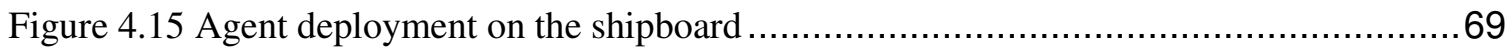

Figure 4.16 Fault occurrence at the feeder level ................................................... 70

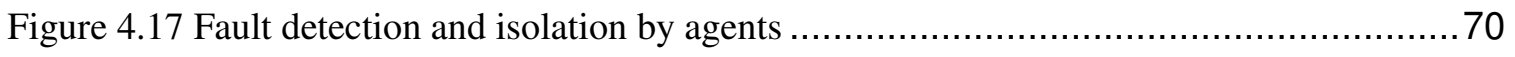

Figure 4.18 Reconfiguration and loads restoration ................................................ 71 
Figure 4.19 Current measurements at the fault location in Feeder\#1, at load\#3 and at the healthy

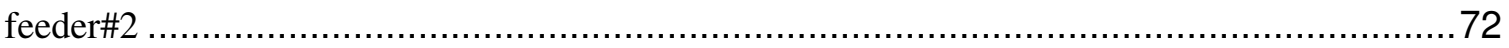

Figure 4.20 Messages exchanged between agents ................................................ 72

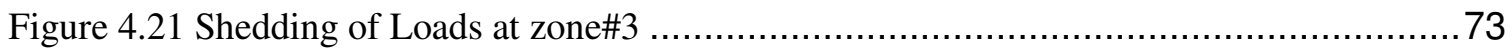

Figure 4.22 Shedding of Loads at zone\#2 …..................................................... 73

Figure 4.23 Power restoration to the loads of zone\#2 .............................................. 74

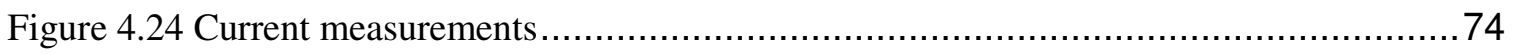

Figure 4.25 Message exchanged between the agents.............................................. 75

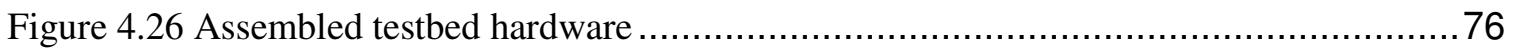

Figure 4.27 Assembled propulsion modules ...................................................... 77

Figure 4.28 Measured current from the testbed .................................................. 78

Figure 4.29 Measured current from the testbed ................................................... 79

Figure 4.30 Voltage profile comparison (fault in Zone3 of CoF) ................................. 79

Figure 4.31 Voltage profile comparison (fault in Zone1 of CoF) ................................... 80

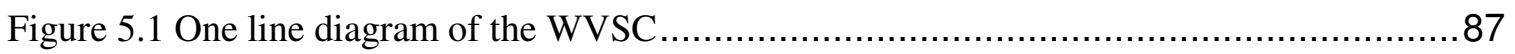

Figure 5.2 Google earth picture showing the WVSC layout....................................... 88

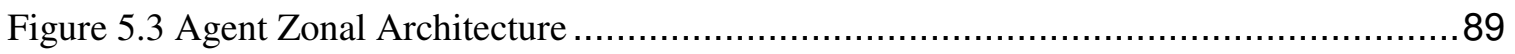

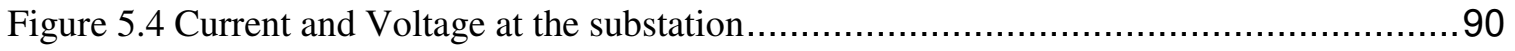

Figure 5.5 Current and Voltage in the faulted zone\#6 .............................................. 91

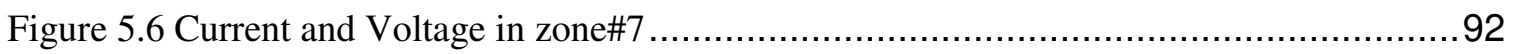

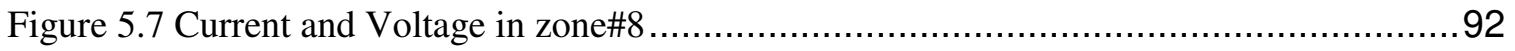

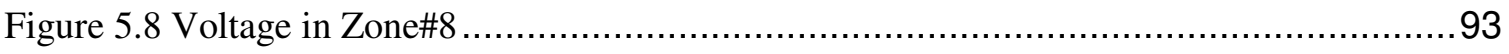

Figure 5.9 Inter Agent Communication.......................................................... 93

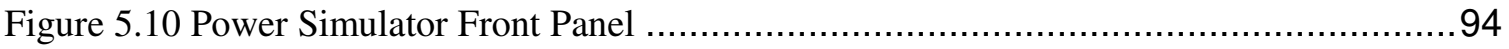

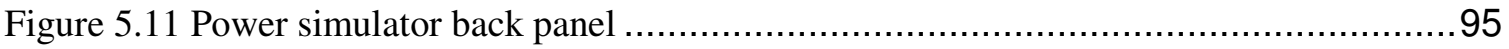


Figure 5.12 One line diagram of Power Simulator................................................ 96

Figure 5.13 Diagram of the CSM-12C32 (Microcontroller module) .................................97

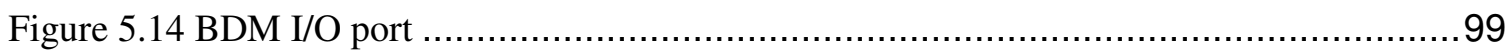

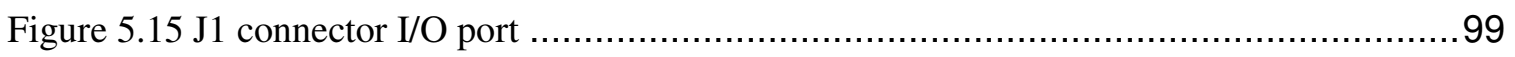

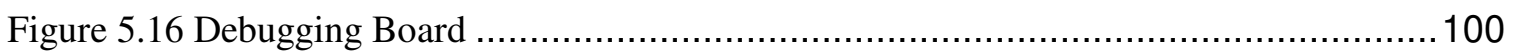

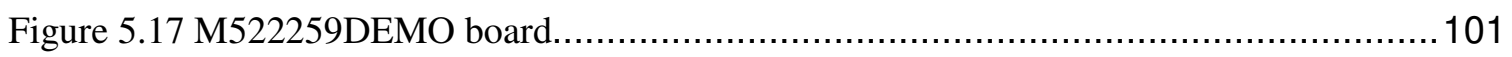

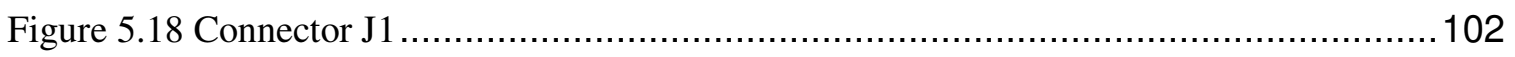

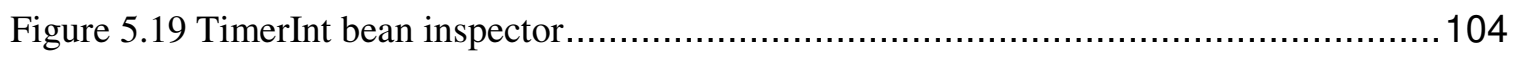

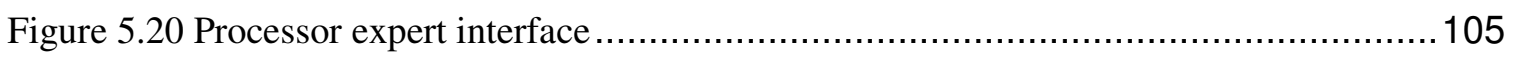

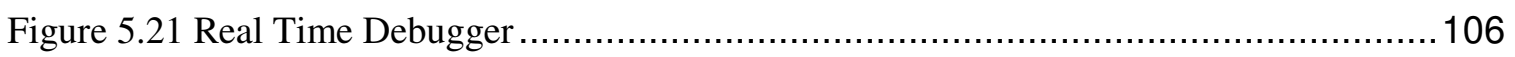

Figure 5.22 Sensor Hardware mounted on an agent module ....................................... 108

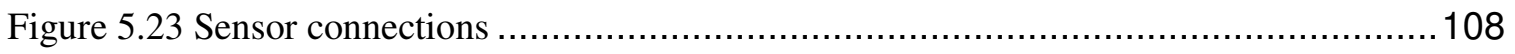

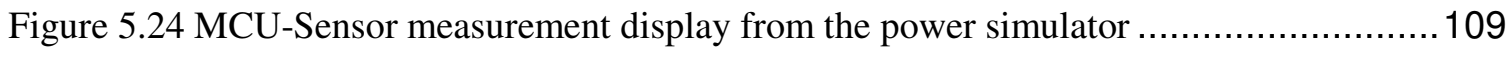

Figure 5.25 Power Simulator Amper Meter .................................................... 110

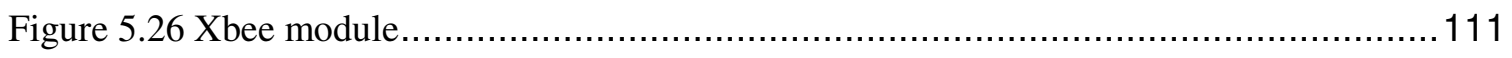

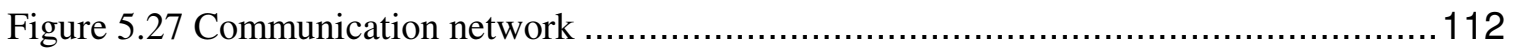

Figure 5.28 Communication Network of MAS for Power systems ...............................113

Figure 5.29 MaxStream's X-CTU Modem Configuration Software ................................114

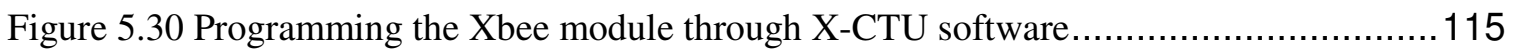

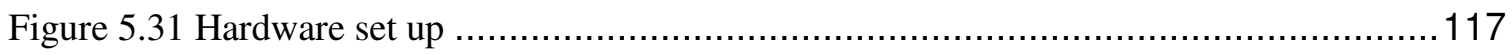

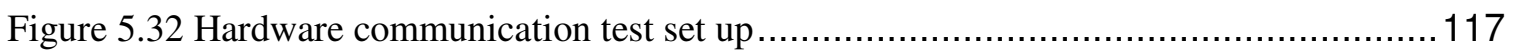

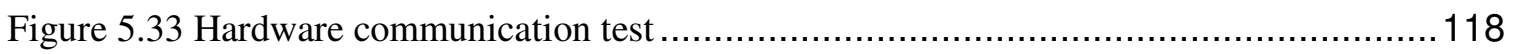

Figure 5.34(a) BTA208X-1000B (b) PC4SD11NTZ ............................................... 119

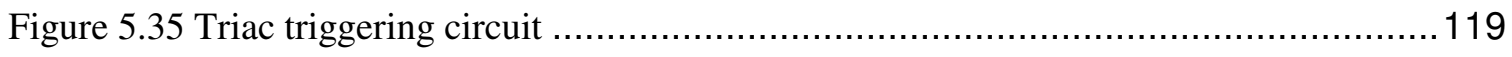

Figure 5.36 Hardware sowing the assembled phototriacs-triacs ................................... 120 
Figure 5.37 Phototriac PC4SD11NTZ characteristic............................................. 120

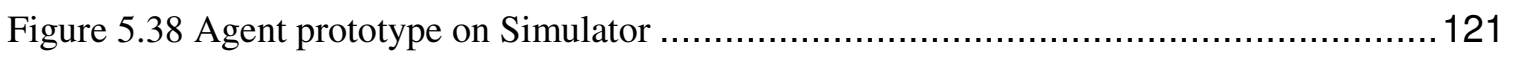

Figure 5.39 Agent prototype on Simulator ....................................................... 122

Figure 5.40 Agent prototype on Simulator ....................................................... 122

Figure 5.41 Simplified one line diagram of the simulator ....................................... 123

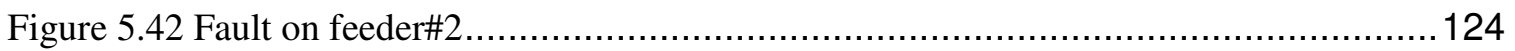

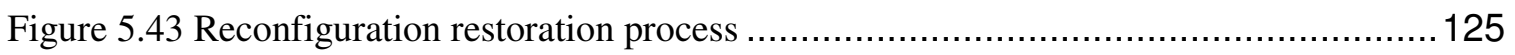

Figure 5.44 Experimental Data of the current on the $1^{\text {st }}$ Feeder .................................... 125

Figure 5.45 Experimental Data of the Current at the load level ...................................126

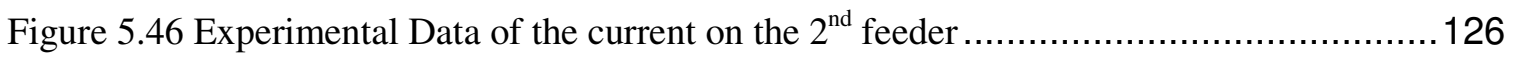

Figure 5.47 Phase current at the substation versus current at the DG ............................. 127

Figure 5.48 Experimental Data of the current waveform at the DG ............................... 127 


\section{LIST OF TABLES}

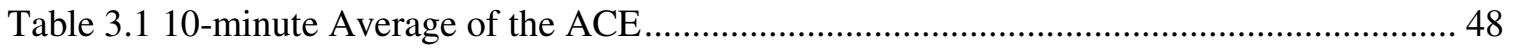

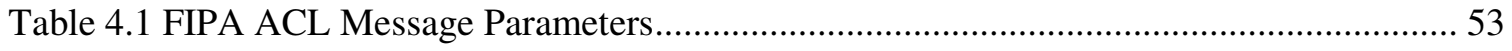

Table 4.2 Power System Immune System MAS analogy ......................................................... 57

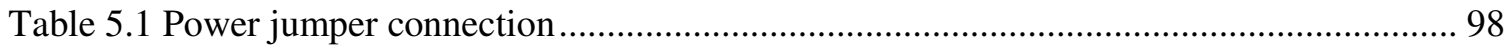

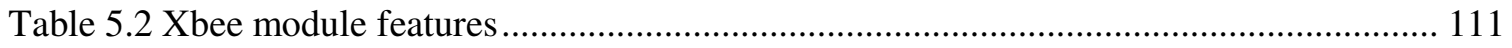

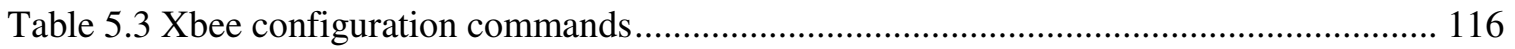




\section{Chapter 1}

\section{INTRODUCTION}

This chapter gives an introduction to the work presented in this dissertation. It is divided into two sections. The first one introduces the work done to solve the load frequency control problem (LFC). Here a novel approach based on the immune system network algorithm is presented. The second part introduces the work done on Multi-Agent Systems design using a new paradigm based on the Immune Multi-Cell System at both the hardware and software level for power distribution system self-healing.

1.1 Decentralized load frequency control design problem

\subsubsection{Problem definition}

For many years, load frequency control problems have been extensively studied by many researchers. The objective is to design an effective feedback controller, which helps a control area maintain the interconnection frequency and power interchanges at their schedules. The turbine and speed governor, which are the prime-mover of the synchronous generator, are controlled by load frequency control to balance generated power and demand; thus the frequency deviation and the net interchange error from the scheduled values remain close to zero. In recent years, the term 
area control error (ACE), which is the combination of area frequency bias $(B)$ times frequency deviation and net power interchange error, is used to measure the power imbalance.

Different types of LFC yield distinct control performances. They depend on the objective and property of control designs. In this work, the goal of load frequency control is to provide robust operation of power system interconnection against possible contingencies. The LFC performance will be assessed by the control performance standards CPS. Furthermore, a desirable LFC strategy should help reduce the unit maneuvering, as well as wear and tear during operation. An ideal load frequency control system which offers all the above advantages is developed in this work.

\subsubsection{Significance of the problem}

For many years, electric utilities have successfully balanced the energy with a simple decentralized control structure. Yet, this structure does not address optimality or economics. In recent years, deregulation has deeply shaped the structure of the electric power systems. As a result, vertically integrated utilities have become a number of independent specialized companies trying to maximize their own benefits. Generation, transmission, and distribution companies make sales and purchases of energy and ancillary services in a free and competitive market under the supervision of the of independent system operator (ISO). Regulation service [1] or load frequency control (LFC), which is an automatic generation control AGC, is one of ancillary services and is very essential for interconnected power system areas. The AGC keeps the system frequency at $60 \mathrm{~Hz}$, and also maintains the net interchange of each control area at the scheduled value. The balance between power generation and demand is achieved through the control of the mechanical power on the shaft of turbo generator by an automatic control of the steam valves or water gates of speed governor, in order to adjust the amount of steam or water flowing into the turbine.

In real world power systems, a proportional-integral (PI) controller is widely employed for the load frequency control purpose. Normally, its control parameters are tuned about once in a month based on trial and error techniques, which may not be efficient for all operating conditions. For this reason a number of contributions from research focused on efficient controller design for load frequency control applications.

In February 1997, the North American Electric Reliability Council (NERC) released new control performance standards [2] CPS1 and CPS2 to assess the effectiveness of the automatic generation control (AGC) of each control area. A control area is defined as [3]: "A control area is an 
electrical system which is bound by interconnect (tie-line) metering and telemetry. A control area continuously adjusts its generation and interchange schedules (via AGC) to match its system load and assists with the frequency regulation of the interconnected power system."

The new standards were adopted to replace the old control performance criteria (CPC), which had been used in the past. The CPC were found to be ineffective standards because when they were satisfied by the control areas, the interconnected system operation was often still poor [4]. Moreover the old standards lacked a technical basis, while the new standards have mathematical support and are based on statistical theory. As a consequence, when they are complied with, the new standards yield a good interconnected system operation. However, regarding CPS data from NERC, some control areas have very good compliance with CPS1, while violating CPS2. These areas are eventually penalized. In order to solve this problem, new control strategies have to be developed.

After deregulation, instead of a single utility having responsibility for load frequency control or regulation service within its territory, many competing companies cooperate and jointly share that responsibility according to contract agreements established within the marketplace. While performing load frequency control, the mechanical equipment of generating units within the speed governor system is constantly moved up and down following the AGC signal obtained from the system operator. Such unit maneuvering causes wear and tear, and hastens the time frame of the maintenance schedule. Consequently, reducing incurred wear and tear is a strategy for cutting costs, a significant objective of those providing the regulation service.

\subsubsection{Proposed approach}

Several centralized and decentralized controllers have been developed. However, most of them are either state feedback or full-order dynamic controllers, which are too complex for industry practices. Therefore, an effective control design that uses the same PI control scheme, and provides robustness and decentralized structure is needed in order to develop a practical load frequency controller. In this work, a novel approach in designing the controller using an artificial immune system theory is presented.

The electric power systems with its complex and large-scale interconnected systems spread over vast geographic areas operating in real-time 24/7 is compared to a human body which never rests, always feeds the cells, heals damaged areas, and protects itself from harmful substances. The power system with its protection scheme and distributed control structure is analogous to a living human body. Anomalies in the system, caused by faults, change in loading conditions, limit 
violations, etc., are similar to pathogens (viruses and bacteria) in the human body that the system strives to eliminate. The controller is designed in a way to manipulate the conventional integraltype load frequency controller to achieve two objectives: (i) minimize equipments' wear and tear and (ii) comply with NERC's CPS1 and CPS2. The proposed controller is totally decentralized using just the measurements of the frequency and the Tie-line power exchange of each area.

\subsection{Electric distribution system self-healing problem}

\subsubsection{Problem definition}

The Electric distribution system is facing a great challenge by responding to the rapidly changing customer needs for electricity in the modern world. The increased use of electricity has increased the number of outages, voltage and frequency violations, and other power quality disturbances.

Power outages alone cost the US 100 billion dollars of economic losses every year. Currently, the electric network is controlled from a centralized control centers. When an electric power supply interruption is caused by a fault, engineers and operators at the command center start working to promptly restore the power system to an optimal target configuration. This process might take for hours in case of distribution systems and days in case of transmission systems. The question that must be raised here is: what would happen if the control center is also affected by the fault? In addition, the restoration process could be a slow and error prone process due to factors such as: a) overwhelming amount of data, b) miscommunications on the part of operators, c) lack of coordination between electric utilities sharing the power grid, or d) lack of accurate measurements. The critical issue is that a slow response during a contingency could generate a cascading effect affecting more portions of the system, and even a total system collapse.

For this reason, the US DoE (Department of energy) identifies dynamic self-healing of the grid as one of the most important characteristic of the modern grid. The challenge is to come up with a self-healing strategy that is distributed, dynamic, intelligent and fault tolerant.

\subsubsection{Significance of the problem}

The Northeast Blackout in 2003 is a very good example of failure of the power grid. This widespread power outage left around 50 million people in Canada and the United States without power and caused about 6 billion USD of financial losses. Hence, the necessity to improve the reliability of the power system due to the increased power demand and congestion issues and to 
mitigate cascading power failures. By reducing power failures and maintaining the voltage profile to the end users, the power quality is enhanced. This can be achieved by finding solutions that are more distributed, automated, and more sophisticated with less human intervention. Furthermore, No issue is getting more attention within the energy industry and among policymakers these days than the Smart Power Grid development. It is one of the top priorities of the United State Department of Energy (USDOE). The USDOE alone is investing billions of dollars into encouraging Smart Power Grids deployment and demonstration through Smart Grid investment grants and Smart Grid demonstration programs. The USDOE has approved around 100 projects in Smart Grid investment grants. The reduction of the number and period of outages is one of the primary goals of the governments. Hence, there is big motivation in solving the problem in the most economical way.

\subsubsection{Proposed approach}

By observing biological systems such as the Human Immune System, we realize that the idea of self-healing following an intrusion of the body by bacteria or virus is an integral part of the immune response. The immune system behaves like a Multi Agent System in which different types of cells constitute different types of agents. The immune system is viewed as a highly distributed, organized and intelligent system equipped with very sophisticated communication systems and learning capabilities, characteristics that are sought in Multi-Agent Systems. The Immune System offers a complete model for designing a robust Multi-Agent System. In this work, an Immune Based Multi Agent System is developed to overcome the drawbacks in the literature in performing self-healing such as voltage violations and fault tolerance. Furthermore, the Multi Agent system is deployed not only on software but also on hardware prototypes to test and validate the results. To fully test the system, a Smart Grid hardware prototype is being developed by upgrading the Power Simulator. Agent hardware modules are being built and deployed on the Power Simulator along with a wireless communication platform and electronic switches as part of the West Virginia Super Circuit Project.

\subsection{Dissertation Outline}

The outline of the remaining chapters is given in this section.

In Chapter2, we are giving a literature overview of the most significant research work in the area of automatic generation control design and multi-agents system application in power distribution systems. 
The human immune system and its components are described in Chapter3 along with its application to the automatic generation control design. The controller is implemented on the two area system and the results are compared to the conventional PI controller.

Chapter4 gives the description of the multi agent system derived by mimicking the Human Immune System behavior. In this chapter the immune system is regarded as a multi-cell system in which the cells cooperate and communicate to achieve the objective of self-healing the body.

In Chapter5, we first give an overview of the concept and the current West Virginia Super Circuit Project, and then we address the design of Agents, sensors, and communication hardware to be integrated with the Power Simulator.

Finally, the conclusion of this study and the future work is detailed in Chapter 6. 


\section{Chapter 2}

\section{LITERATURE REVIEW}

This chapter presents a comprehensive overview of the previous research work carried out on load frequency control design and multi-agents and its applications in the area of power distribution system fault detection restoration and reconfiguration.

\subsection{Power System Load Frequency Control Design}

This Section summarizes the ideas from existing work and publications related to load frequency control designs and strategies in power systems. Then, the contributions of this part of the dissertation, which have been designed mainly to improve upon previous work, are presented. A large power system interconnection consists of a number of control areas interconnected through tie lines. Their fundamental role is to balance power generation and constantly changing demand, in order to maintain the interconnection frequency and net interchange at their scheduled values. The control area operator performs load frequency control (LFC) through the automatic generation control (AGC) system. In industry, a PI controller is used to automatically compensate the difference between generation and load. The controller uses the area control error (ACE), which can be obtained by measurements of interconnection frequency and net interchange, as input. The PI controller is usually tuned once awhile online by trial and error, which obviously may not be efficient in a wide range of operation. 
In the work presented in [7], Genetic Algorithms (GAs) are used to tune and search of the integral controller parameter by minimizing a given performance indices. This study investigates two performance indices. The first one is the integral of square of error (ISE) which tends to penalize all errors with respect to given weighting factors. The other performance index is the integral of time multiplied absolute value of the error (ITAE) [8]. It includes the time factor in order to penalize the settling time of the controlled system GAs used. However, such an optimization needs to be performed through a number of simulations of the entire system, which require physical parameters for all control areas, and consequently might not be practical. In addition, the performance indices used include the frequency deviations of both areas, which is not preferable from a decentralized control point of view.

In [9] the authors formulate an AGC simulation framework accounting for LFC contracts for a twoarea power system. This paper also proposes an optimization based on a trajectory sensitivity approach to obtaining the optimal parameter for the integral load frequency controller. In this work, the two areas are assumed identical, and the performance index used for optimization by a gradient Newton algorithm.

In the above mentioned works, the optimization methods used require lengthy simulations and need physical parameters for entire system to find the optimal control parameters of LFC controllers. In practical implementations, parameters of one control area are unlikely to be available to the other areas.

Using a centralized controller that relies on global information would not be practical. Electric power systems are complex networks spreading over vast geographic areas and comprising a number of subsystems or control areas interconnected to one another by tie lines.

Thus the decentralized control scheme that results in a controller, which needs only local information in controlling its own subsystem, is very desirable for the control design of power systems.

In the work performed in [10], [11] and [12] the authors apply the robust $H_{\infty}$ control theory to obtain robustness for the closed-loop system against uncertainties such as demand changes and area interconnections. The approach is too complex to implement in real world power system. A systematic based decentralized load frequency control design is presented in [13]. For each subsystem, a local observer is developed to reconstruct global states. Those estimated states are used as the inputs of the proportional-integral (PI) controller, which formulates the decentralized control scheme. However, the PI controller used is much more complex than the traditional one, which requires only the ACE as the 
input. Also it results in the need for a state observer, which actually increases the order of the control system.

In [14], a decentralized controller based on singular perturbation methods is proposed. In this method a decoupling is obtained by assuming that power systems are relatively weakly coupled systems [15]. With the approximately decoupled subsystem, the optimal decentralized controller is designed, using the linear quadratic regulator (LQR) technique. In reality, the interconnections among power systems can be from weak to very strong depending on the distance between them. Consequently, the proposed decentralized controller may not perform well in medium or strongly coupled power systems.

The authors in [16] apply an inclusion principle with the emphasis on state observer and optimal controller in their design. In their work, the state space model of a two-area power system is formed. It is subsequently cast into a new expanded system that has a larger size, and contains all necessary information about the original system. Afterwards, the corresponding subsystems of the original system are extracted from the new system. Using a linear quadratic Gaussian (LQG) technique, decentralized controllers are finally designed for the individual resulting subsystems. According to the proposed control scheme, the order of the observer based controller increases with the size of the subsystem depending on modeling details and the number of generating units. Moreover, the complexity of using an overlapping decentralization technique would increase exponentially with the number of subsystems.

A robust decentralized controller for the LFC is presented in [17] and [18]. The authors use the Riccati equation approach. In this work the proposed controller requires the measurements of all local states, and some of them are not measurable. Therefore, a state observer is needed, which increases the complexity of the control system when the number of states becomes large.

Intelligent control such as neural networks and fuzzy logic has also been applied to this problem. In [19], a fuzzy logic LFC controller is designed to replace the conventional one. The fuzzy rules are designed with expertise to regulate the ACE to zero. The frequency deviation and its rate are used as the inputs of the fuzzy logic system, whose output is the control signal of a control area.

In the study done in [20], the uncertainties of system parameters are taken into consideration, including power system time constant, frequency bias constant, and synchronizing power coefficient. Artificial Neural Networks (ANN) are used to adapt control gains to variations of parameters such as power system time constant and synchronizing power coefficient in order to maintain good dynamic performance. The training sets are generated by solving the state-space LQR problems for different values of the parameters. By doing this the number of training set becomes very large in order to 
increase the performance of the controller which makes the network training very slow. Some authors [21] try to solve this problem by replacing the Neural Network block in [20] by a fuzzy system as a gain scheduler sine it needs a fewer training sets. In these papers, all optimal feedback gains are designed for the whole system rather than for individual control areas, which do not address the decentralized control scheme.

Most of the research work discussed is mainly employed to increase the dynamic performance of the LFC control operation. During the last few years the objective of the LFC operation has changed to accommodate system deregulation and NERC's new control performance standards. In the past, the LFC was provided by vertically integrated utilities (VIUs) for their own territories. After deregulation, it has become possible for different generation companies (Genco) and independent power producers (IPP) to participate in providing this service instead of the VIUs, which have become transmission companies (Transco). The main idea is that LFC becomes a commodity which can be sold and purchased in the market. Gencos and IPPs may choose either to sell it by submitting their bids to the independent system operator (ISO) or not to participate if the profit opportunity is rare. In such an environment, all market participants tend to maximize their benefits rather than enhancing power system reliability. The ISO, which is the middle man, needs to supervise and approve the actions of those market players to assure the reliability of the power system operation [22].

Especially for regulation service, the North American Electric Reliability Council (NERC) employed the control performance criteria (CPC), which include A1 and A2, for three decades to specify whether an area's generation is sufficiently controlled to make the interconnection frequency and net interchange meet their schedules. Criterion A1 requires that the ACE should cross zero at least once every ten minutes. Criterion A2 requires that ten minute averages of ACE should be less than an area specific parameter $(L d)$. However, using these criteria, it was found that when they were satisfied, interconnected system operation was often poor.

In February 1997, new control performance standards CPS1 and CPS2 are developed from mathematical relations between the ACE and the frequency error. These standards are adopted to replace the old criteria in order to eliminate the CPC's drawback [23]. CPS1 is a limit on the 12-month average of the product of the ACE and frequency error from its schedule. CPS2 is similar to A2, but its specific limit is determined based on a statistical method and is much larger than A2's. From observations, CPS1 and CPS2, which have technical background support and are more flexible than the old criteria, allow less unit maneuvering, resulting in a saving of fuel costs 
An attempt in enhancing the economic operations of the AGC as required by NERC is presented in [24]. In this work a logic based control is developed to regulate interconnection frequency and lower unnecessary generation maneuvering, resulting in a reduction of unit wear and tear.

This technique aims at keeping the ACE within a near-zero target boundary, but unconditionally forcing a small ACE to return to zero does not optimize operating objectives, and may not be economically optimal. In addition the authors fail to address the issue of compatibility with NERC's new control performance standards.

The work done in [25] addresses the NERC's new standards as logic based AGC. The logic system is composed of CPS1 and CPS2 control modules, which need to be coordinated orderly. The compliance factor of each CPS1 and CPS2 is defined and calculated every 4 seconds.

The concept of the proposed logic is that as long as those compliance factors are less than their corresponding thresholds, the AGC signal to regulating units is set to zero, which means no control actions are induced. Otherwise, the AGC signal is set to the average of the raw ACE.

Determination of the threshold values is very important, since they directly indicate the control tightness. In this paper, those thresholds are selected by searching for the values that result in the best compliance with CPS1 and CPS2. However, those values are constant and specifically chosen for the test system and would not be applicable to other power systems.

\subsubsection{Contribution of the work to the LFC Problem}

From literature review, we can see that a big number of studies involving the LFC have been carried out in the last three decades. However, existing robust load frequency controllers are relatively complicated and impractical for implementation. The power industry is very conservative and would stay away from any complicated controller. Some studies employ centralized control, which rely on global measurements or high-order observers. This approach cannot simply be implemented because of deregulation. Thus, an effective robust decentralized LFC controller which is attractive from implementation point of view and uses the same trusted PI structure needs to be developed. Furthermore, the replacement of the old control performance criteria (CPC) with the new control performance standards (CPS1 and CPS2) by NERC significantly influences control areas, requiring the development of new LFC strategies. In a market based environment, such strategies should result in not only compliance with CPS, but also in saving operational and maintenance costs for power producers. The main objective of this work is to fulfill the above mentioned requirements by:

- Developing a novel control strategy based on the Immune Network Theory. The non-willingness of industrial policy makers to change the type of controller is forcing us to keep the same PI type control 
structure. In this work, while keeping the PI type controller a robust decentralized load frequency control designs which result in effective performance and simple controller structure is designed.

- Developing LFC strategies in compliance with NERC's new control performance standards CPS1 and CPS2. According to the historical data, some control areas often violated the standards, and they were penalized by NERC.

- Develop operational and control strategies that help generating units or generation companies in cutting down their operational and maintenance costs. Those costs partially result from inefficient control actions, which cause excess unit maneuvering and unnecessary reversals of unit equipment.

\subsection{MAS for power system self-healing}

Multi agent systems have been applied to several areas in power systems, such as reconfiguration and restoration, fault detection, protection coordination, voltage stability control, reactive power control, electricity market pricing etc. These theoretical based researches are being applied, at present, to terrestrial power systems to test the reliability of MAS.

There are many reasons for automating the power grid. The main reasons for automation are to improve the reliability of the system and to ensure the continuity of the service by reducing the human intervention. Manually performing complex circuits restoration switching or reconfiguration without overloading the lines might take a long time. The solution is to use an intelligent technique which allows us to perform these tasks quickly and accurately.

The reconfiguration and restoration problem is a combinatorial problem due to many combinations of switching operations that increase with the growing complexity of the system. Several techniques, such as mathematical programming [26], heuristics [27], have been proposed in the literature to solve this type of problem. In [26], the restoration is targeted as an integration of Mathematical Programming with Expert System. The objective function is solved efficiently by decomposition-coordination strategy. They solve the restoration problem by using a two-step process. Firstly, the target configuration is determined and then sequence of control operations of the switches is determined based on the target configuration. In [27], a heuristic approach based on the immune system algorithm is used. This technique aims at performing the reconfiguration process while optimizing for voltage profile, power losses, and number of switches operated. The approach is implemented on meshed power network.

Most of these approaches are centralized and depend on a powerful computing and communication infrastructure to handle the huge amount of data. In this era of deregulation, it is difficult to solve this problem by using conventional and centralized techniques since it requires a coordination of different parties such generation companies, consumers and independent system 
operators. Furthermore, the incorporation of Distributed Energy Resources (DERs) in the grid has added more complication to the system. The main reason for using DER is that it serves as a local generation when there is a power supply shortage. However, adding and interconnecting DERs to the power network give rise to system stability problems and new protection coordination schemes must be developed. In addition, centralized approaches are subject to a single point of failure which decreases the reliability of the overall system.

The Multi-Agent System (MAS) technology is a branch of distributed intelligence that deals with concentrating the intelligence at the device or component level. By making the components of power systems intelligent, we shift the conventional centralized approaches to distributed and localized approaches thus reducing the human intervention. For example, by making a DG intelligent, which means that it has a knowledge capability, it can perform a self-control by deciding whether to connect to the grid or not based on communication with other intelligent entities connected to the generation side or transmission side and have some key information about the state of the system like voltage profile or stability of the system.

Many authors have conducted research in the area of reconfiguration and restoration of power system. The author in [28], [29] and [30] proposed an autonomous distributed multi-agent model for power system restoration. Starting with [28], he introduced Bus Agents (BAGS) in the system to find the optimal target network configuration. In [29], he introduced a single Facilitator Agent (FAG) to act as a manager in the decision making process in addition to the BAGs. Then, in [30] he developed a system that not only solves the problem of minimizing the de-energized loads, but also ensures that each agent solves its own bus's problem autonomously. These are achieved by two kinds of agents in the system, namely, Bus Agents (BAGs), relevant to the substation bus concerned, and Junction Agents (JAGs), corresponding to the junction of a multi-terminal transmission line. The purpose of the BAG is to restore the load directly from its own bus. The JAGs operate to control the cooperation and competition between BAGs connected through a junction. Both the BAGs and the JAGs have their own strategies for restoration. Message communication between agents is achieved by general-purpose Agent Communication Language (ACL) which is mounted on Extensible Markup Language (XML). This method also assumes that the configuration is radial. In this work, the use of a single facilitator agent centralizes the systems, thus failure of this central point brings down the entire system. In [31], the author proposed a new decentralized multi-agent approach for the bulk power system restoration to enhance the speed of autonomous decision making process. The system is constructed with two- 
level hierarchical architecture. In the upper level, several Local-area Management Agents (LMAs) and Remote-area Management Agents (RMAs) are implemented while several Load Agents (LAGs) and Generator Agents (GAGs) are located at the lower level. The LMAs have two objectives: to restore within their local areas and to interact with other LMAs in order to achieve the overall objective. A RMA has only one objective, which is to assist the restoration of the loads within the remote area. This has to be achieved by series of negotiations between the RMA and the neighboring LMAs. LAGs and GAGs correspond to load management system and generator management system which store their respective loads' and generators' local information. In addition to their local communication, they also interact with the upper level agents.

Apart from these, the same authors also proposed several agent methodologies to address the issues in Bulk Power system restoration. In [32], the authors have introduced a single Independent System Operator Agent, several Local Area Management Agents (LMAs), Local Facilitator Agents, Generator Facilitator Agents, Remote Load Facilitator Agents, Load Agents and Generator Agents to address the entire bulk power system.

Contribution towards bulk power restorations have also been made in [33]. The authors proposed a hierarchical architecture for MAS bulk power restoration. There are two major agents, Management Agent (MGAG) and Practical Components Agents (PCAG) in a Power System. PCAGs constitute of several Generator Agents, Substation Agents and Load Agents. MGAG is generated for a particular system to be in charge of the subsystems and administer the negotiations between the other agents to check for constraint violations and to facilitate path search for restoration.

Some authors have presented the restoration of power system by distributed reconfiguration [34]. In their multi-agent model, they have introduced three types of agents, named Switch Agents (SAs), Substation Breaker Agents (SBAs) and Tie Breaker Agents (TBAs). The objective in this work is to detect and isolate the fault and supply as much load as possible, while assuming that the components are not overloaded and radial configuration is maintained. Fault detection is based on monitoring the fault currents of neighboring agents. The power system model was developed using Virtual Test Bed (VTB) to simulate the P-Q model of the three phase power system and the agent model was developed in Matlab. 
In a later work [35], the same authors presented a better model for the restoration problem. This model addresses the load priority, limitations on generating source capacity and cable transfer capacity. However, this paper also assumes that the configuration is radial. The agent model was developed in JADE with Switch Agents (SAs), Load Agents (LAs) and Generator Agents (GAs) and the power flow is calculated by forward and backward propagation. These agents communicate only with their neighboring agents. The interface between JADE and VTB had been developed by Florida State University for this system.

The work done in [36] presents another approach for reconfiguration of electric power distribution system. In this work, the power system simulation is done using Matlab-Simulink and JADE was used to develop the agent model. The major concentration of this paper is given to the integration of these two software models. The importance of the middleware is to let the MAS sense when a fault occurs in the power system and to make necessary control actions. The communication middleware allows the JADE MAS to send and receive data to and from the Simulink power system model by using Transmission Control Protocol (TCP) sockets. This model consists of a Server Agent, Zone Agent and a Communication Agent. The same authors have modeled the MAS as an Intelligent Power Router (IPR), where the intelligence of IPR is provided by the software agents [37]. These power routers are deployed in the power distribution network for reconfiguration and re-routing the power flow the same way as routers do in the information distribution network. Based on their model requirements, FIPA Contract Net Interaction Protocol is used. In the Contract Net Interaction protocol, an initiator agent corresponds with the responders in order to perform its tasks. The responders may send a proposal or refusal according to their system conditions at that time. The negotiation continues with the responders who send proposals. After receiving all the proposals, the initiator selects the best proposal according to its criteria and informs the relevant responder with the acceptance message. Those responses that are not selected will be informed with a rejection message by the initiator. Then the respective responder will perform its intended task in order to achieve the overall objective of the system.

The work discussed above for multi-agent reconfiguration of power system assumes that the system topology is radial at all times. In [38], the authors attempted to address the mesh power network. In this method, there are several rounds of negotiations along the ring configuration addressing the source generation capacity and the load demands. The series of accumulation of 
messages during multiple negotiation rounds causes Redundant Information Accumulation (RIA), leading to an unstable information flow. The authors propose a reconfiguration method to avoid RIA where the algorithm generates a tree structure to break the existing rings. The simulation was done using JADE as the agent platform and Real Time Digital Simulators (RTDS) as the power system modeling tool. The agents developed in JADE have been implemented in iPAQs, which are pocket Personal Computers, and communicate with RTDS through a Field Programmable Gate Array (FPGA) interface.

In [39], the author attempted to implement a MAS on the Southern California Circuit of the Future. The system they used could be operated in loop for optimal reconfiguration. This approach is based on an ad hoc way to reconfigure the system where each and every possible configuration is applied, and then the best switching configuration is chosen. The switching sequences are performed in MATPOWER software then is fed to the agents. The reconfiguration problem is a combinatorial problem; therefore, using this approach would result in huge computations. Furthermore, the configuration target is found by running load flows of each switching sequence separately on different software, then the solution is sent to the MAS to be applied which makes the whole idea of using MAS absurd in this case. The authors in [40] used maximum flow algorithm to decide if a reconfiguration target can be applied. Since the maximum flow formulation does not take into account the voltage profile, the authors use DEW software to check for the voltage profile after a reconfiguration target is chosen. This approach is not realistic since a single agent doesn't have a global information or voltage profile of the entire system.

\subsubsection{Shortcomings in the literature}

In most of the work on performed on power distribution system reconfiguration and restoration using MAS, the decision or the solution is found by relying on communication and negotiation of the agents to trace back potential sources of power in an ad hoc way. The agents look for a path to restore the power without any prior knowledge on the effect of their actions on the voltage profile or on the grid since they just perform power management. While it might work for relatively small radial networks where no voltage violation or drop is expected, it is clear that for relatively large distribution systems, finding a path without checking for voltage violations and losses might not yield an acceptable solution especially in pick load. Agents should have a priori knowledge of the voltage profile resulting from the reconfiguration they propose. In other words, the voltage profile plays an important role in choosing a target configuration for power restoration. 
Furthermore, if the system is not radial it becomes very complex to find a target configuration relying on communication alone, since the power does not flow in only one direction as in a radial system. In addition, most of the research involves somehow a centralized part that is vital to the operation of the MAS such as Facilitator Agent or Global Agent. Most of the works that addressed the issue of voltage violation assume that the agents have access to global information about the voltage profile to check is their choice violates the voltage limits. Obviously this approach is not practical since the agents have limited information about the system.

In conclusion, we can summarize the major shortcomings in the literature:

- Centralized Simulation of the MAS using computer based platforms (Jade). This approach tends to overlook the problems associate with the meshed two way communication system which is in its self a major source of challenging problems.

- Most of the work is performed on radial systems while a high penetration of DGs are expected in Smart and modern grids.

- In simulation, test systems are assumed balanced in order to use single phase analysis tools while distribution systems are inherently unbalanced. This assumption makes it impossible to analyze the response of agents to different types of faults.

- Most of the work on power management is initiated without taking into account the voltage profile, which gives rise to problems in long feeder lines like the WVSC ones.

- Use of centralized portion that are vital to the operation such as global agent and facilitator agent.

- Lack of experimental or laboratory testing. Hence, the difficulty in comparing different approaches and a real assessments of the techniques.

\subsubsection{Contribution of this work in MAS design}

- This work proposes a new way of designing multi agent systems based on natural human Immune System MAS for power systems reconfiguration, restoration and load shedding in which the millions of cells form the agents that strive to heal the system.

- The technique takes advantage of a heuristic approach in looking at the optimality of the configuration target in terms of voltage and losses by using the Clonal Selection Algorithm as the brain of the agent, and the distributed nature of the MAS in adding the real time action and the smart communication between the components. 
Chapter 2: Literature Review

- This algorithm can be applied to both meshed and radial power systems with or without DGS.

- Real time interaction of the Multi Agent System on a three phase dynamic model of the power system.

- The work goes one step ahead in filling up the gap from previous research in deploying the MAS designed on hardware prototypes for experimental validation of the technology. 


\title{
Chapter 3
}

\section{A DECENTRALIZED IMMUNE BASED}

\author{
CONTROL DESIGN OF AN AUTOMATIC
}

\section{GENERATION CONTROL IN COMPLIANCE WITH THE NERC's STANDARDS}

\subsection{Introduction}

Biology has been an inspiration to numerous computational artificial intelligence, such as genetic algorithms, neural networks, and the one addressed here, the immune based system. This human immune system is a complex system of cells, molecules and organs with the primary function of protecting the body from attacks by eliciting an immune response. The attack in this case is by pathogens or infectious agents, such as bacteria and viruses. The immune system mimics a control strategy in an electric power system setting. 
Electric power systems are complex, large-scale interconnected systems spread over vast geographic areas. They operate around the clock and electricity is just-in-time commodity. In the framework of the approach presented in this work, the power system, with its protection scheme and distributed control structure, is analogous to a living human body. Anomalies or disturbances in the system, and deviations from normal operating conditions, are then seen as pathogens or damages that the system strives to eliminate. Using this concept of immune cells-pathogens the power system control design is addressed in general, and in particular the automatic generation control problem is used to illustrate this approach and demonstrate its feasibility. In this chapter, first a brief description of the biological immune system is presented and explained in a war-like setting, and simple examples in power system terminology and optimization are given. Second, the automatic generation control design problem is cast as an immune based optimization problem. A two-area power system is used to demonstrate the effectiveness of this novel approach.

\subsection{The Immune System}

In this section a brief overview of the biological immune system is presented to help understand the rest of the chapter. The emphasis is on the key components of the biological immune system that exhibit learning and intelligence.

\subsubsection{Biological Immune Systems}

The defense system of the human body against external harmful attacks can be thought of as an organized army that protects the body from external enemies. This analogy is used to explain the biological immune system. In case of an attack by external enemy cells such as bacteria and viruses, it becomes a multi-faceted war where the main role of the elements fighting in the front line is to prevent the enemy cells from affecting the body. The immune system is usually seen as a great protector from microscopic organisms that can cause death and destruction to the body. In Figure 3.1, the important organs of the human body that contribute to, and form, the immune system are shown. 


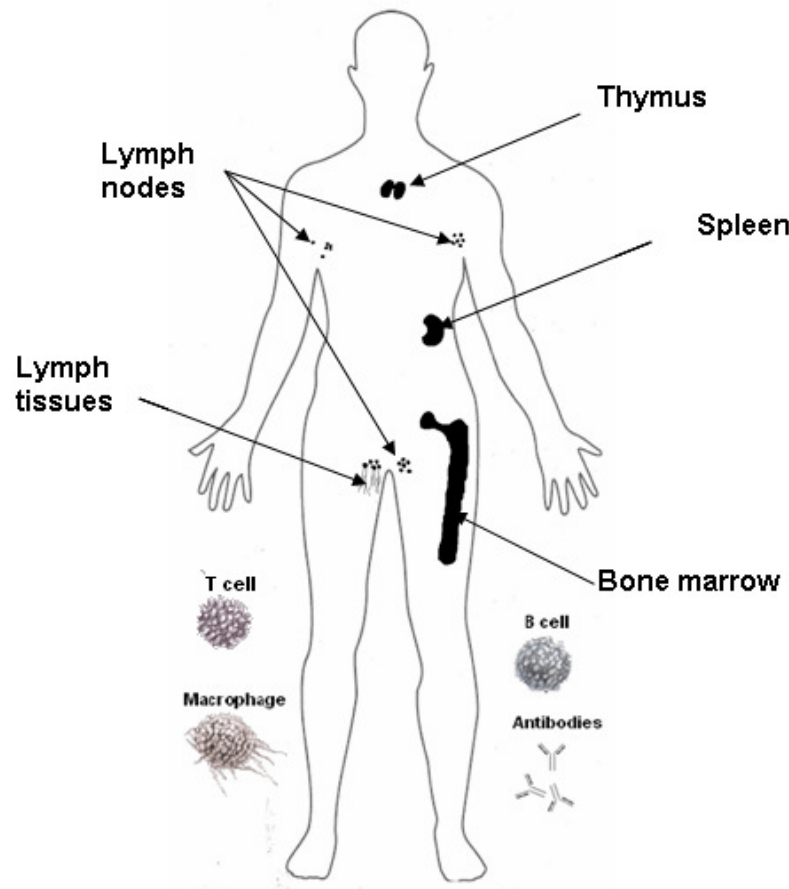

Figure 3.1 Immune System Components

When we think of the immune system, the ideas of adaptation, learning and cognition seem to be natural, but where does the learning or the intelligence come from? If we think about it a little bit more, we realize that we all had experiences with this idea of the immune system learning and improving over time. For example, scientists usually inject a preparation of inactive cancer cells, or proteins that are unique to cancer cells, into a person who has cancer and try to train the person's immune system to recognize the living cancer cells and attack them. So, how is it that by exposing the immune system to a small disease it will later be able to protect us completely from exposure to that disease? How is the immune system capable of rapidly defeating previously seen, and even unseen but similar, bacteria or viruses unless the Immune System has a memory and learns, making it an adaptive control system.

The functions of the immune system are divided into two main activities (1) recognition and (2) response [41]. First, the immune system has to distinguish between the pathogens that invade the body (nonself) from its own components (self). Second, once the pathogens have been recognized, the immune system mounts an effector cell response to destroy the pathogens.

The human body is therefore like a besieged castle surrounded by countless enemies and microorganisms. Nevertheless, it is protected by a very organized and intelligent system. The first barriers to these foreign enemies are the physical layer such as skin and the chemical barriers 
such as the ones secreted in the nasal mucous. If these front line barriers are breached the pathogens have to deal with the innate or natural adaptive immune system.

The innate immune system does not discriminate between pathogens. It is mediated by agents roaming scavengers like macrophages. The macrophages roam around the body to detect and eliminate pathogens. The detection of pathogens is made possible because the chemicals they produce are not produced in large quantities within the host organisms [42]. Thus, as soon as a macrophage detects a foreign organism, it engulfs the foreign organism and consumes it in a process known as phagocytosis as illustrated in Figure 3.2 After phagocytizing the antigen, the macrophage conveys alert signals, or information about the antigen, to other agents to start the attack. Signals from macrophages activate the other agents such as cytokines. These agents act as further alert signals that influence the behavior of the immune system components against pathogens.

An example of this process in power systems could be the identification of the type of disturbance or fault, which is needed to stimulate a specific strategy for solving the problem.

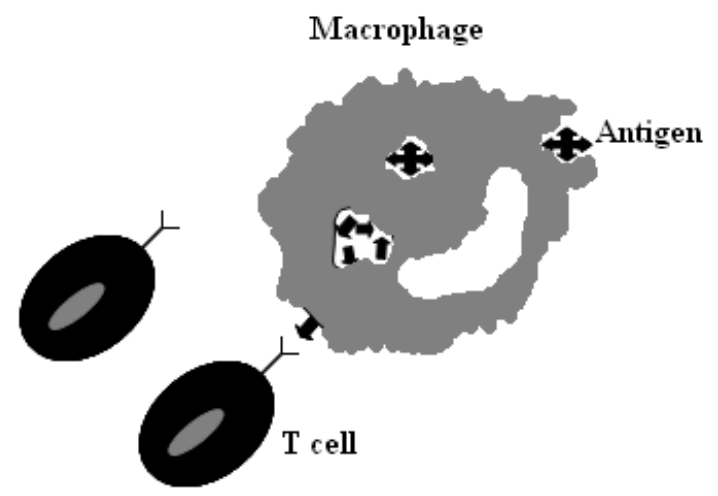

Figure 3.2 Macrophage engulfing antigen

The non-specific mechanism of recognizing pathogens by the innate immune system implies that the innate system is not able to detect the vast number of pathogens that invade the body. For this reason, the human body is equipped with a more sophisticated system which is the adaptive immune system that can handle pathogens not detected by the innate immune system.

The adaptive immune system is distinct from the other layers of the immune system due to its own distinguished features, not previously encountered, that include defense against specific foreign organisms, ability to learn and remember information about foreign organisms. The main agents of the adaptive immune system are the B-cells and T-cells. B-cells and T-cells are 
lymphocytes or white blood cells that are produced by stem cells in the bone marrow. Here, stem cells rapidly pass through a number of biological stages and take on a completely new structure by turning into lymphocytes. (A stem cell is an unspecialized cell that gives rise to a specific specialized cell, such as a blood cell.)

\subsubsection{The Antibody factory: the B Cells}

Some of the lymphocytes produced in the bone marrow depart when they mature and become fully functional, and are transported to the lymphatic tissues through the blood. These lymphocytes are called the B cells. B cells are the weapon factories of the body and they produce the proteins, called antibodies, which are meant to attack the enemy.

The cells undergo a highly complex and laborious process to become B cells. These cells must first pass a severe test in order to become the warriors who will protect human health. In their initial phase, the B cells rearrange the gene fragments that will form an antibody molecule. These genes are actively transcribed as soon as the rearrangement is complete. Only comprised of a compact nucleus and very little cytoplasm initially, the B cells undergo unbelievable changes when they meet an antigen. They divide repeatedly and build up thousands of assembly points in their cytoplasm for the manufacture of antibodies, as well as an extensive channeling system for the packaging and exporting the antibodies. One B cell can pump out more than 10 million antibody molecules in an hour.

Some B cells become "memory cells". These cells do not immediately participate in the body's defense, but keep molecular records of past invaders in order to accelerate a potential war in the future. Their memory is very strong. When the body meets the same enemy again, this time it is rapidly geared to the appropriate weaponry production. Thus, defense becomes faster and more efficient.

\subsubsection{The B cell recognition of the antigens}

In a complete state of preparedness for war, the B cells then learn to discriminate the enemies from the body cells before defending the body. They do not need to expend much effort to do 
this, because these cells and the antibodies they manufacture are able to recognize the enemy directly from their shapes without any assistance. A receptor on their surface meets the antigen for which it was programmed, and binds to several small parts on it. Thus, the antigen is identified as a foreigner. In this way, B cells can easily recognize antigens, such as bacteria.

\subsubsection{The function of B-cells}

B cells are like guards who are always on the lookout for microbes. When they encounter an invader, they rapidly divide and start to produce antibodies. These antibodies bind to microbes like B cell receptors. The enemy cells that are marked by the antibodies as foreigners are driven out of the body at the end of the relentless struggle of phagocytes and T cells. By the time the B cells inactivate the enemy with the millions of antibodies they have produced, they have also marked it for killer cells. Here, there is another important point, which is as important as destroying and marking foreign cells. It is about how so many antibodies can be produced by a limited number of genes. The B cells use the genes in the human body to manufacture antibodies. However, the number of genes in the human body is less than the number of antibodies produced. This situation causes no problem for the cells. Despite all these limitations, they succeed in producing nearly 2 million antibody types an hour. B cells interact in various combinations with available genes to make the above-mentioned production.

\section{Example of B-cells for Power Systems:}

Let us design an example of B cell for power systems. The first thing to do is to define where the actions can be applied to the system. For example, we can act on relays and breakers to change the configuration of the system, we can also act on the controllers at the generation level to increase or decrease the power generation to track the demand, etc.

We mentioned earlier that B cells are the weapon factories of the body and they produce the proteins, called antibodies, which are meant to attack the enemy. Consider the simple power system shown in Figure 3.3. The system is composed of one main generator and an emergency generator, four transmission lines and two loads. In this case we can design our B cell by simply creating a protein (antibody) from the collection of all the inputs, where actions can be applied, to the power system in question. We can define for example a protein by combining the following actions:

- Connect Generator 1 or disconnect Generator 1 
- Connect Generator 2 or disconnect Generator2

- Increase generation or decrease generation

- Shed or decrease load 1 or connect or increase load 1

- Shed or decrease load 2 or connect or decrease load 2

- Energize line 1, 2, 3 or 4

- Disconnect line $1,2,3$ or 4

- etc.

Initially the two $20 \mathrm{MW}$ loads are fully supplied by the main generator through the transmission lines numbered 3 and 4 . These lines have a $20 \mathrm{MW}$ capacity and therefore they are fully loaded. Suddenly, a fault occurs that causes the loss of line number 4. At this time all the $40 \mathrm{MW}$ generated by the main unit is flowing over line number 3 and still both loads are supplied. However line number 3 is now overloaded and cannot operate under this condition for a long time. Recognizing this anomaly, the immune-like system will react to resolve this problem. A B cell can then be stimulated as shown in the figure below.
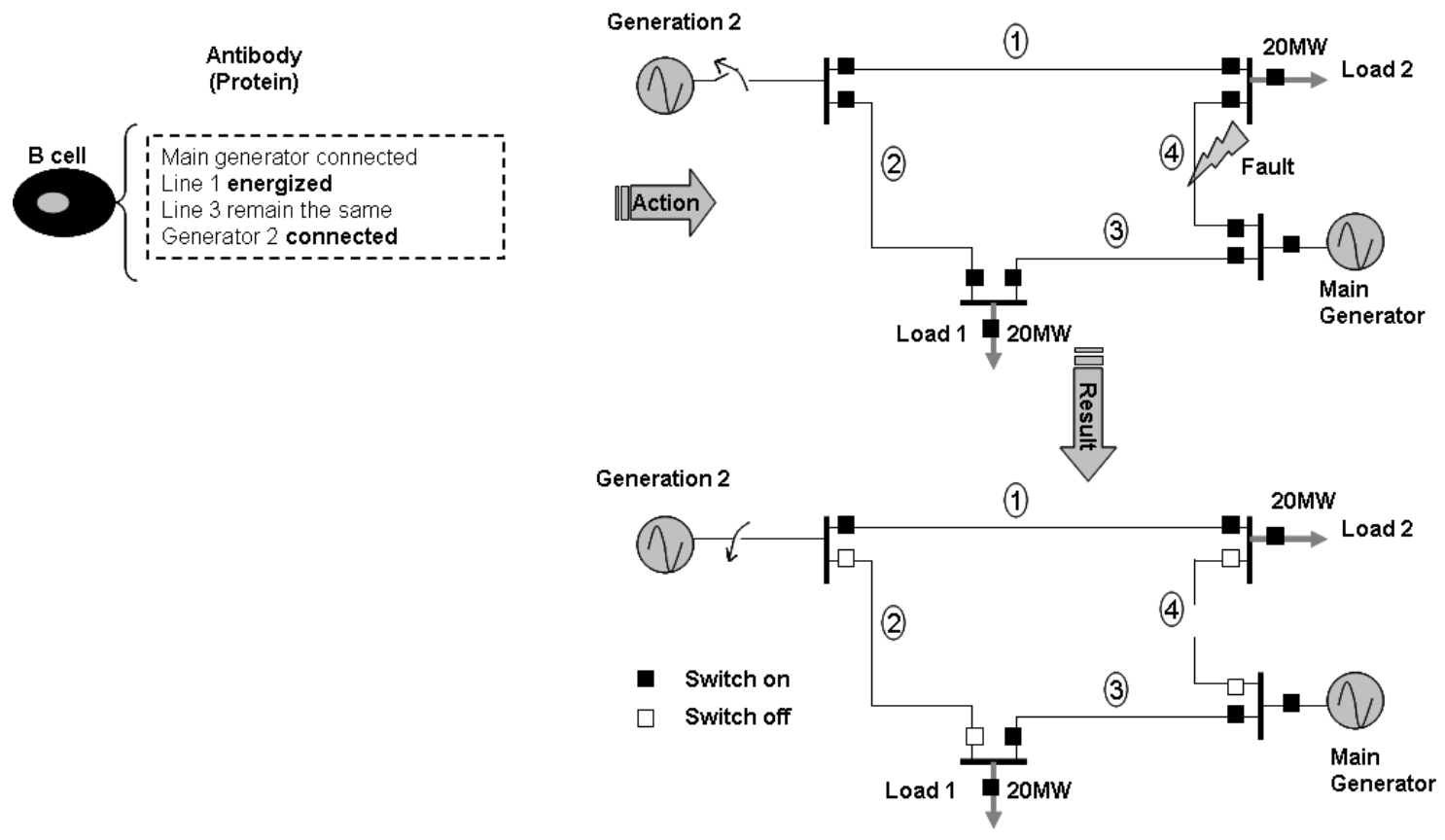

Figure 3.3 Stimulated B-cell acting on the power system

The solution in this case, which can be seen by arranging the genes of the protein, is to obtain 20 MW from generator number 2, which is now connected and to disconnect line number 2. 


\subsubsection{The T Cells}

Some lymphocytes migrate to the thymus after they are manufactured in the bone marrow. The lymphocytes, which multiply and mature here, are called $\mathrm{T}$ cells. These cells mature to form two different types: killer and helper $\mathrm{T}$ cells. After a three-week education, $\mathrm{T}$ cells migrate to the spleen, lymph nodes, and intestinal tissues to wait for the time of their mission.

In comparison to $\mathrm{B}$ cells, $\mathrm{T}$ cells must go through a much more complicated course to be ready to begin their mission. Just like B cells, they, too, are simple cells in the beginning. These simple cells go through a series of difficult tests to become a $\mathrm{T}$ cell.

In the first test, it is checked whether the cell can recognize the enemy or not. The cells recognize the enemy with the assistance of "MHC" (Major Histocompatibility Complex) located on the surface of the enemy, which is a molecule that subjects the antigen to a series of chemical processes and presents it to the T cells. Eventually, only those cells that are able to identify the enemy can survive. The others are not tolerated and they are immediately destroyed.

The recognition of enemy cells alone does not ensure the survival of $\mathrm{T}$ cells. These cells must also have a very good knowledge of the harmless substances and the regular tissues of the human body so to as prevent unnecessary conflict, which will eventually harm the body.

\subsubsection{Antibodies}

Antibodies are protein-structured weapons, which are manufactured to fight against the foreign cells entering the human body. These weapons are produced by the B cells. Antibodies destroy invaders. They have two main functions: The first is to bind to the invader cell, which is the antigen. The second is to decompose the biological structure of the antigen and destroy it.

Swimming in the blood and non-cellular fluid, antibodies bind to disease-causing bacteria and viruses. They mark the foreign molecules to which they bind, so that the body's fighter cells can distinguish them. This way, they also inactivate them. An antibody fits the enemy (antigen) perfectly, just like a key and a lock assembling in a three dimensional structure.

The human body can produce a compatible antibody for almost every enemy it encounters. Antibodies are not of one type only. According to the structure of every enemy, a specific antibody powerful enough to deal with it is produced. This is because an antibody produced for 
one disease may not be effective for another. This is like manufacturing a compatible key for each of millions of locks straight away. What is important is that the manufacturing agent does this without examining the lock or using any mold. It knows the formula by heart.

Manufacturing a specific antibody for each enemy is rather an unusual process, which deserves a closer attention. This process can be realized only if the B cells know very well their enemies and their structures. There are, however, millions of enemies (antigens) in nature.

The cell combines the hundred thousand genes it contains in different combinations to form new antibodies. It receives the information from some genes and combines it with the information in other genes and makes the required production according to this combined information. $1,920,000$ different antibodies are formed as a result of 5,200 different combinations. An unlimited number of combinations can be made with the use of one hundred thousand genes. The cell, however, uses, with great intelligence, only 5,200 basic combinations and produces 1,920,000 specific antibodies.

\section{Example 2: Gene Combination in Power Systems}

An example of gene combination is given in the Figure 3.4 bellow.

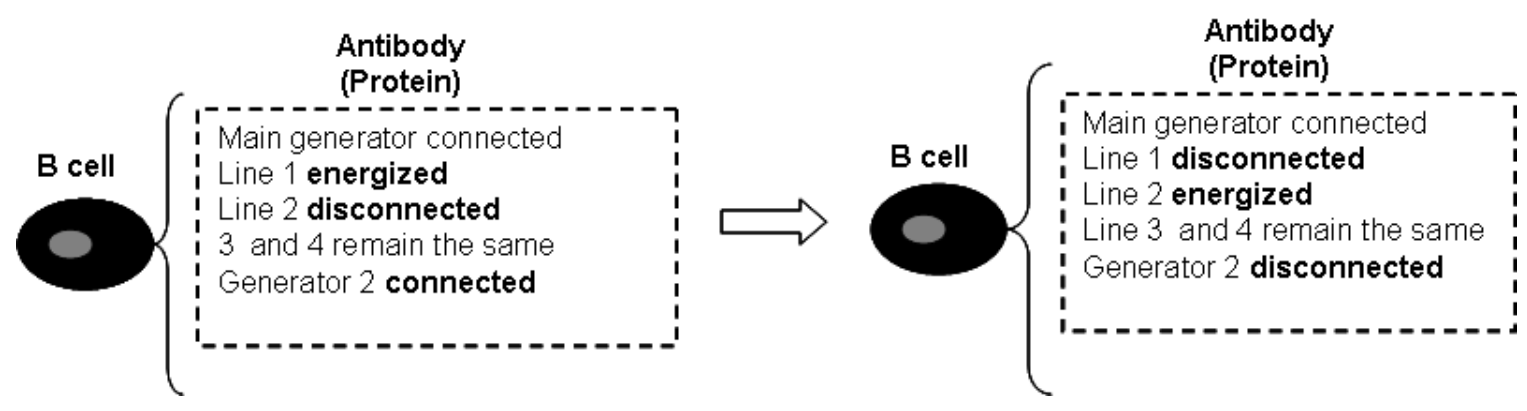

Figure 3.4 Example of gene combination in power systems

An individual B cell makes copies of its own specific antibody and attaches them to the outer surface of its cell membrane. The antibodies extend outward like highly tuned aerials waiting for contact with the specific bit of protein that they can recognize. An antibody is made of two light and two heavy chains of amino acids held together in a Y shape as illustrated in Figure 3.5. The constant regions of chains are the same in many different types of antibodies. But the variable regions - the tips of the arms - each have a uniquely shaped cavity that fits exactly onto the shape of the antibody's "chosen" protein fragment.

In Figure 3.5 we show an example of a binary model of the antibodies which can be used for engineering purposes. Each variable $(x i)$ encoded by a pre-defined number of bits is separated into light chain and heavy chain mimicking the structure of antibody in biological immune 
systems as depicted in the Figure 3.6. The length of each chain is determined by the user-defined light-heavy chain-length ratio.

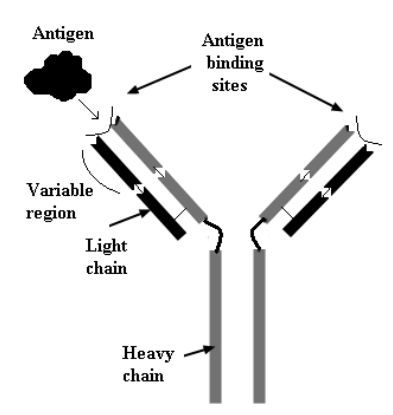

Figure 3.5 Antibody molecule

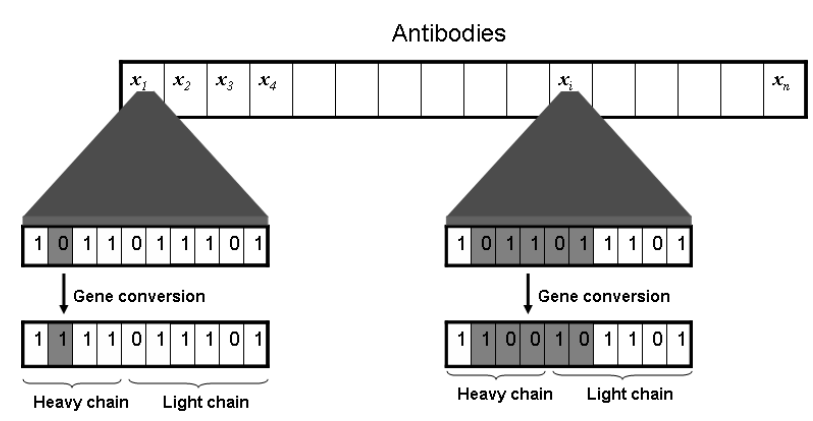

Figure 3.6 binary model of Antibodies

\subsection{Clonal Selection theory}

Several different proposals have been made on how a given B-cell interacts with an antigen and how this interaction affects the other components of the immune system. Clonal-Selection theory is one such idea [43]. It proposes that as an antigen enters the immune system certain B-cells are selected based on their reaction to this antigen to undergo rapid cloning and expansion. As an antigen is presented to a B-cell, the B-cell's antibodies react in some degree to the antigen. This reaction is often termed the affinity of that B-cell (or antibody) for the given antigen. Those Bcells with a sufficient affinity are allowed to produce offspring in relation to their degree of affinity as illustrated in Figure 3.7. This allows for the rapid expansion of cells that can successfully attack the invading antigen. Some B-cells transform into plasma cells which produce a concentration of free-antibodies that works with T-killer cells to neutralize the antigenic threat. Once the threat subsides, certain B-cells which were highly stimulated by the antigen are selected 
to become long lived memory cells which allow for a more rapid secondary response if a similar antigen is encountered in the future

As previously mentioned, B-cells are selected, based on their affinity to an antigen, to produce a number of clones. The purpose of this process is first these clones are produced in order to attack or neutralize the invading antigen. Those cells that have a higher degree of affinity are allowed to produce more clones since these cells will be able to more appropriately respond to the invading antigen. The second purpose of clonal production is to develop immune cells that are more adept at recognizing and reacting to the antigen. This is done through a process of affinity maturation through mutation. Each offspring of a B-cell can potentially be a mutated variety of its progenitor. B-cell offspring undergo mutation based on inverse proportion to their affinity values. That is, those cells which have a higher affinity value mutate less than those cells with a lower affinity value. Through this process, the affinity of subsequent generations of B-cells will be greater in reaction to the antigen, and more diversity will also have been added to the system through the wider exploration afforded by the high mutation rates of the cells with lower affinity measures.

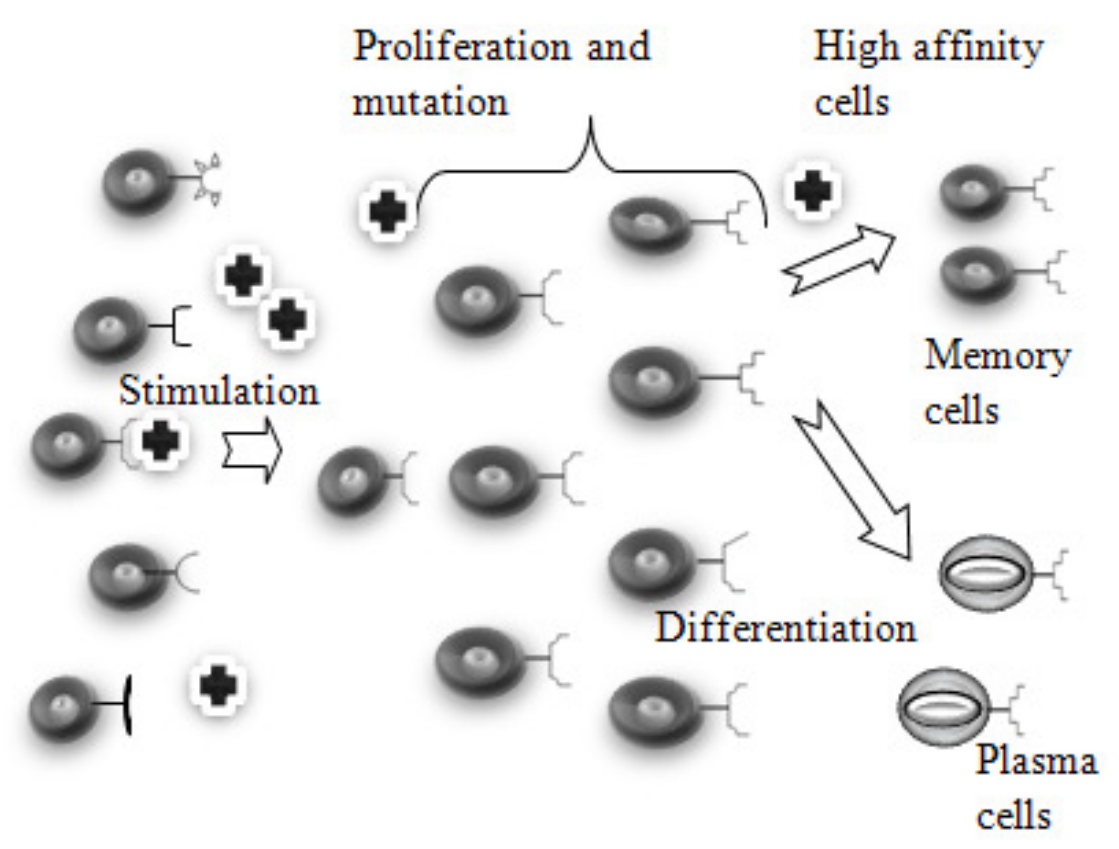

Figure 3.7 Clonal selection principle

\section{Example 3: Optimization with Clonal Selection}


Here is an example of how to implement the Clonal Selection for an optimization purpose. Let's try to maximize the sinusoidal function $f(x)=\sin (x)$ where $x \in\left[\begin{array}{ll}0 & \pi\end{array}\right]$.

We can choose to code the interval of search in binary representation of 6 bits. Each element of the interval can be considered as an antibody.

The antigen that stimulates the B-cell in this case can be the function $f(x)$ we want to maximize. Let us choose randomly 2 antibodies $\mathrm{Ab} 1$ and $\mathrm{Ab} 2$ representing $x=0.5$ and $x=1.2$ as illustrated in Figure 3.8a. As mentioned before, those cells that have a higher degree of affinity are allowed to produce more clones. Since $\sin (1.2)>\sin (0.5)$ implies that Ab1 will have more clone than Ab2 as shown in Figure 3.8b. Now we have our clones, we can perform a mutation as seen in Figure 3.8c. Notice here that the mutation on the antibody Ab1 takes place in Light chain which results in a local search of the maximum. On the other hand, the mutation on Ab2 is taking place in the Heavy chain which results in a global search this allows us to explore the search domain as in Figure 3.9.

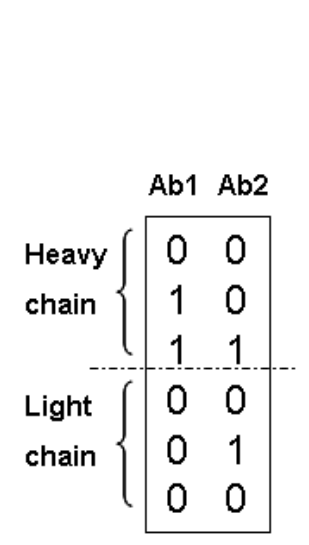

(a)

\begin{tabular}{|c|c|}
\hline$\overbrace{\mathrm{c1} \mathrm{c2} \mathrm{c3}}^{\begin{array}{l}\text { Clones } \\
\text { of Ab1 }\end{array}}$ & $\begin{array}{l}\text { Clones } \\
\text { of } \mathrm{Ab} 2\end{array}$ \\
\hline $\begin{array}{lll}0 & 0 & 0\end{array}$ & 00 \\
\hline $\begin{array}{lll}11 & 1\end{array}$ & 00 \\
\hline $\begin{array}{lll}1 & 1 & 1\end{array}$ & 11 \\
\hline $\begin{array}{lll}0 & 0 & 0\end{array}$ & 00 \\
\hline 000 & 11 \\
\hline 000 & 00 \\
\hline
\end{tabular}

(b)

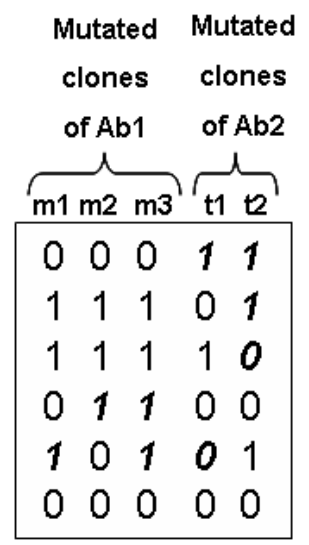

(c)

Figure 3.8 Mutation process of the antibodies 


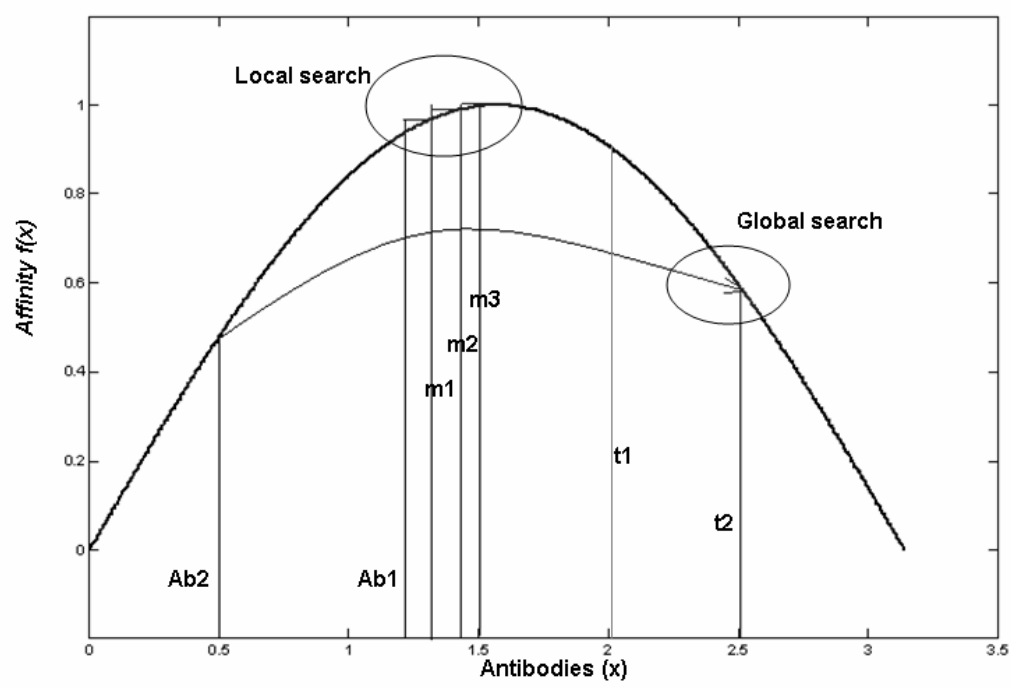

Figure 3.9 Antibodies performing search for the max of $f(x)=\sin (x)$

\subsubsection{Immune Memory and Learning}

In the previous sections we just explained how the cells react to incoming antigen, but we didn't really how, when presented with the same (or similar) antigen, the immune system responds much more efficiently and rapidly. The question is how, then, is memory maintained?. One the accepted notion is that, after the initial exposure of the immune system to a given antigen, a longlived memory cell is maintained in the system. Upon subsequent exposure, this memory cell is allowed to produce clones much more rapidly than typical B-cells in reaction to the antigen or structurally similar antigens. So, immune memory is maintained through a collection of memory [44].

\subsection{Immune Network Theory}

The Immune network theory was first introduced by Jerne in 1974 [45]. It models the immune response in terms of a network of antibodies that stimulate and suppress each other [46]. Following, are some of the terms used in this theory. Epitope is the antigenic determinant that a B-cell looks for, while paratope is the receptor on the B-cell that locks on to a specific epitope. Also, all B-cells contain structures similar to antigenic epitope, displayed by various regions of antibody molecules. They are called idiotypes. An idiotope is a single idiotypic epitope. The immune system is considered to be an enormous and complex network of paratopes that recognize sets of epitopes, and of idiotopes that are recognized by sets of paratopes. Thus each 
element in the network can recognize and can be recognized. Such a network is illustrated in Figure 3.10.

When an antigen invades an immune system, those antibodies, whose paratopes can recognize the antigenic epitope, bind to the antigen. These antibodies also have idiotopes, which are recognized by other antibodies. Thus those antibodies that recognize these idiotopes, bind to the antibody and suppress it. The response of the network depends on how well an antigen is recognized and on the stimulation and suppression mechanisms of the network.

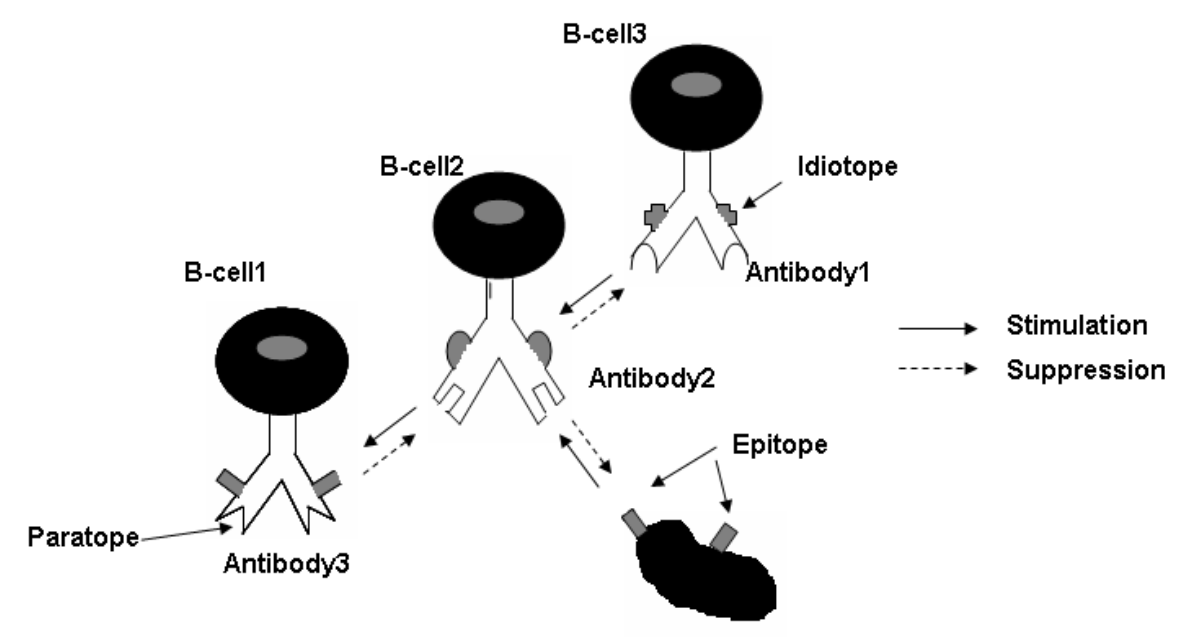

Figure 3.10 Antibody network representation

Antibodies that recognize either the antigen or other antibodies and lock on to them, effectively suppress those antigens and antibodies. On the other hand, the antigens and antibodies that have idiotopic structures complimentary to paratopes of antibodies are said to stimulate those antibodies. The extent of idiotopic locking determines the mutual affinities antigen-antibody affinity and antibody-antibody mutual affinity.

\subsection{Computational Aspects of the Immune System}

Immune system has an inherent distributed nature and parallel processing capabilities. It also possesses powerful information processing capabilities. Following are some of the most important computational aspects of the immune system [47].

1. Pattern Recognition - Immune system can recognize and classify different patterns in epitopes and idiotopes to generate selective responses. Self - non-self determination is achieved by this pattern recognition capability.

2. Feature extraction - Antigen Presenting Cells (APCs) digest the antigen and extract significant features from the antigen molecules to be presented to the B and T-cells. 
3. Diversity - a diverse set of B-cells is required for the antigen recognition process, which is done in parallel at various sites throughout the immune system.

4. Immune Memory - immune system possesses a content-addressable memory, which contains genetic information about antigens experienced, in the form of matured memory cells.

5. Reinforcement Learning - B-cells that can recognize antigens proliferate and multiply while those that cannot identify the antigen are destroyed and undergo genetic editing. This is similar to reinforcing desirable actions in reinforcement learning.

Some of the other computational aspects are noise immunity, distributed detection, self-regulation (through T-cells), threshold mechanism and probabilistic detection, adaptability, specificity.

\subsection{Power System - Human Body Analogy}

Electric power systems are complex, large-scale interconnected systems spread over vast geographic areas that operate in real-time $24 / 7$ just like the human body which never rests, always feeds the cells, heals damaged areas, and protects itself from harmful substances. The power system with its protection scheme and distributed control structure is analogous to a living human body. Anomalies in the system, caused by faults, changing loading conditions, and structural changes such as loss of generating units or power lines, etc., are similar to pathogens (viruses and bacteria) in the human body that the system strives to eliminate. The human body uses immune system component which are B-cells, T-cells, etc. Similar aspects are developed for power systems that are proper design strategies or combination of actions by control and protection devices. The Figure 3.11 bellow shows the analogy between human body-pathogens and power systems-anomalies. 


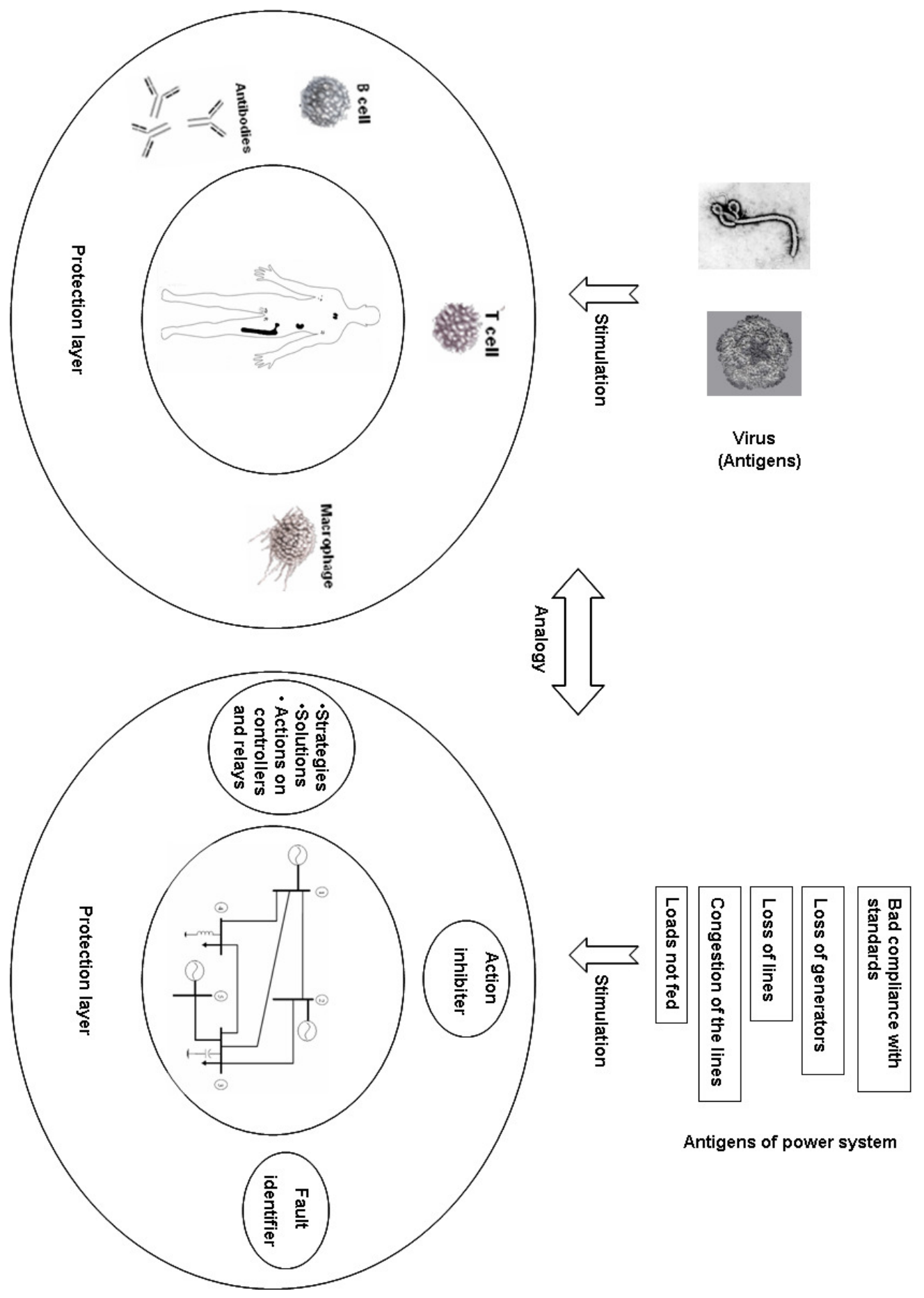

Figure 3.11 Human body-power systems analogy 


\subsection{Automatic Generation control (AGC) problem}

The purpose of the previous section was to set the theme for the development of learning algorithms. We have seen that the immune system does have mechanisms that facilitate learning in some fashion. Through exposure to antigens, the immune system is capable of remembering the exposures, generalizing beyond one specific antigen, and reacting in a more appropriate manner when presented with the same or similar antigens. It automatically improves with experience. In the following, we turn our attention to the application of all this biological behaviors in power systems and specifically to the energy balancing problem in power systems, known also as the Automatic Generation Control (AGC) or Load Frequency Control (LFC).

\subsubsection{Introduction and background}

For many years, electric utilities have used successfully balanced the energy with a simple decentralized control structure. But this structure does not address optimality or economics. Hence, the objective of this work is to utilize the standard control structure but to add the effects of re-structuring and aim at minimizing wear and tear of the equipment while complying with the criteria imposed by the North American Electric Reliability Council (NERC). Restructuring of the electricity industry has forced vertically integrated utilities that own generation, transmission, and distribution systems to split into independent and more specialized companies. New participants have emerged to compete in the generation business and to provide ancillary services such as regulation and load following. To benefit fully from this environment, market participants have to minimize their operating and maintenance costs associated with generating units' maneuvering. Energy balancing or regulation is provided by the load frequency control mechanism (LFC), which is an automatic control of the generators' set points.

The power system consists of several interconnected balancing areas. Each one is responsible for tracking its native load and provides contracted power with its neighbors. This balance is monitored with a single signal called the Area Control Error (ACE) which accounts for errors in the interconnection frequency $(\Delta F)$ as well as errors in the interchange power over tie lines with neighboring areas, i.e. the tie-line power error $(\triangle P$ tie $)$. The ACE is given by:

$$
A C E_{i}=\Delta P_{\text {tie }}-10 B_{i} \Delta F
$$


where $(\Delta F)$ is the interconnection frequency error, i.e. deviation from the nominal frequency, and $\left(\Delta \mathrm{P}_{\text {tie }}\right)$ is the tie-line power error, i.e. deviation from the contracted power interchange. $B_{i}$ represents the control area's frequency bias expressed in $\mathrm{MW} / 0.1 \mathrm{~Hz}$, it represents the sensitivity of the loads with respect to frequency changes. A block diagram of a two-area interconnected system is shown in Figure 3.12.

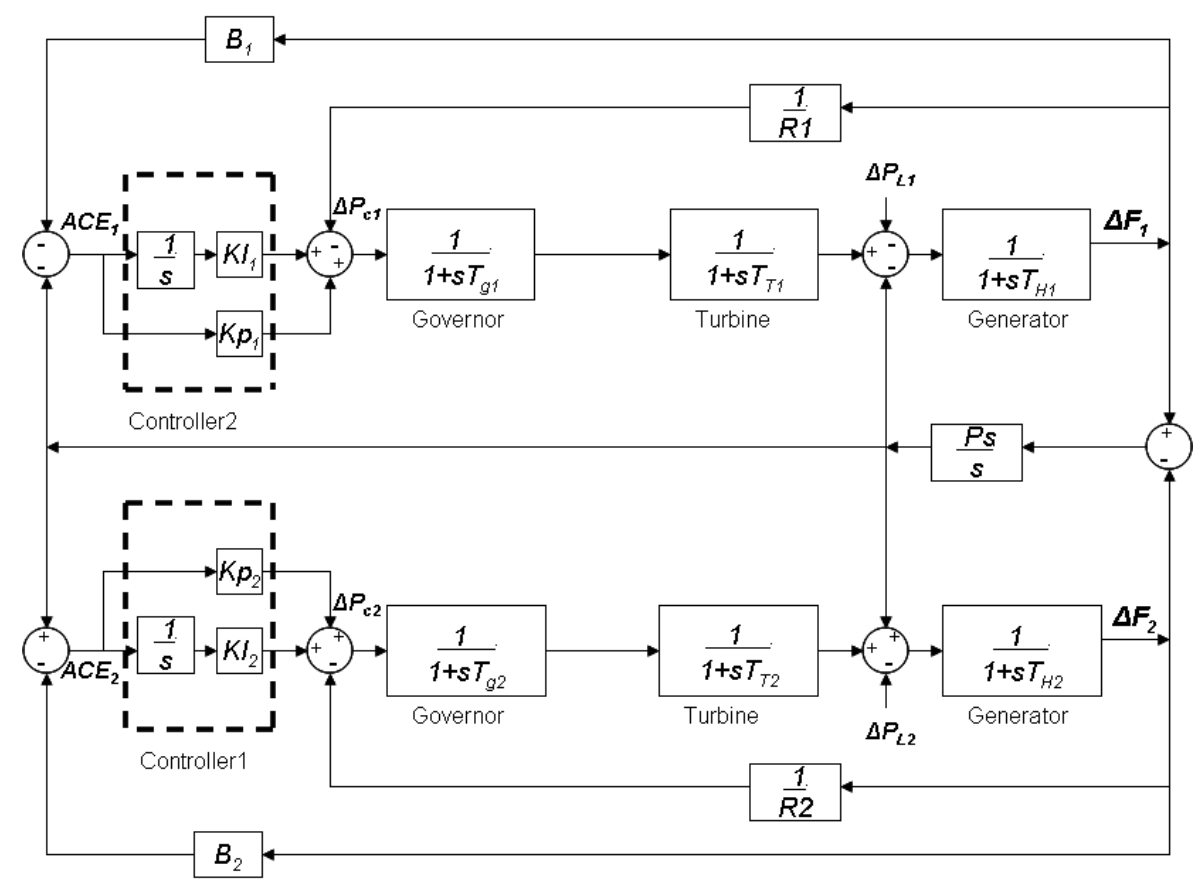

Figure 3.12 Two area system model with AGC loop

Conventional LFC uses a feedback signal that is proportional either to the integral of ACE (Integral Controller) or to the ACE itself and its integral (PI controller). These feedback signals are used to maneuver the turbine governor setpoints of the generators so that the generated power follows the load fluctuations. However, continuously tracking load fluctuations definitely causes wear and tear on the equipment, shortening their lifetime, which can be very costly. Here a novel load frequency controller is presented. It is manipulated by an Immune based controller which is designed to reduce wear and tear of the equipment, and to assure its control performance is in compliance with NERC's control performance standards, CPS1 and CPS2. The LFC control structure is selected to be consistent with general practices and would be feasible for implementation. This control structure is a decentralized, integral-type controller whose parameter is automatically tuned using the proposed controller. The control parameter is reduced to diminish high frequency movement of the speed governor's equipment when the control area 
has high compliance with NERC's standards. When the compliance is low, the control parameter is raised to the normal value. Nonlinear simulation results, comparing the proposed methods to conventional designs, illustrate the effectiveness of the proposed scheme.

\subsubsection{NERC's control performance standards}

For equitable operation of the interconnected system, control areas have to comply with NERC's control performance standards CPS1 and CPS2 which were adopted in February 1997. Each control area is required to monitor its control performance and report its compliance with CPS1 and CPS2 to NERC at the end of each month [48].

\section{CPS1}

CPS1 assesses the impact of ACE on frequency over a 12-month window or horizon. It is defined as follows: over a sliding 12-month period, the average of the "clock-minute averages" of a control area's ACE divided by "ten times its area frequency bias" times the corresponding "clockminute averages of the interconnection frequency error" shall be less than the square of a given constant, $\varepsilon 1$, representing a target frequency bound. This is expressed by:

$$
A V G_{12-m o n t h}\left[\left(\frac{A C E_{i}}{-10 B_{i}}\right)_{1} * \Delta F_{1}\right] \leq \varepsilon_{1}^{2}
$$

Where

$\Delta F:$ interconnection frequency error,

$B_{i}$ : frequency bias of the ith control area,

$\varepsilon_{1}:$ targeted frequency bound for CPS1,

( ) 1 clock-1-minute average.

To calculate CPS1, a compliance factor (CF) and a 1-minute average compliance factor (CF1) are introduced:

$$
C F=A V G_{12-m o n t h}\left[C F_{1}\right]
$$

Where:

$$
C F_{1}=\left[\left(\frac{A C E}{-10 B}\right)_{1} *\left(\frac{\Delta F}{\varepsilon_{1}^{2}}\right)_{1}\right]
$$

CPS1 is then obtained from the following equation: 
Chapter 4 A Decentralized Immune Based Control Design of an Automatic Generation Control

$$
C P S 1=(2-C F) * 100 \%
$$

To comply with NERC, CPS1 should not be less than $100 \%$.

\section{CPS2}

The second performance standard, CPS2, limits the magnitude of short term ACE values. It requires the 10-minute averages of a control area's ACE be less than a constant $\left(L_{10}\right)$ given in eq.5

$$
A V G_{10-\min u t e s}\left(A C E_{i}\right) \leq L_{10}
$$

where:

$$
L_{10}=1.65 \varepsilon_{10} \sqrt{\left(-10 B_{i}\right)\left(-10 B_{s}\right)}
$$

Where $B_{s}$ is the summation of the frequency bias settings of all control areas in the considered interconnection, and $\mathcal{E}_{10}$ is the targeted frequency bound for CPS2. To comply with this standard, each control area must have its compliance no less than 90\%. A compliance percentage is calculated from the following equation:

$$
\text { CPS } 2=\left[1-\frac{\text { Violations }_{\text {month }}}{\text { Total periods }- \text { Unavailible periods }_{\text {prof }}}\right]
$$

Where Violations $_{\text {month }}$ is a count of the number of periods that the clock-10-minute averages of ACE are greater than $L_{10}$ in one month.

\subsection{Immune based optimization using clonal selection theory}

In this section, the AGC controller in this section is a feedback signal proportional to the ACE and its integral, i.e. a PI (Proportional Integral) control signal. The clonal selection theory is used to find the optimal proportional and integral gains for each control areas. The controllers are decentralized, as each area uses only the local measurements. The objective is to regulate the Area Control Error (ACE) which represents a combination of frequency and tie-line power deviations. The variation of the ACE indicates that an imbalance in energy is taking place and must be regulated. Thus the performance index in each area is chosen to be of the following form [49]:

$J=\frac{1}{2} \int_{0}^{\infty}\left(q A C E^{2}(\tau)+r \Delta P_{c}^{2}(\tau)\right) d \tau$ 
Chapter 4 A Decentralized Immune Based Control Design of an Automatic Generation Control

Where $q$ and $r$ are weighing coefficients.

The objective of the Immune System algorithm in this case is to perform a heuristic search for the best combination of gains that recognize the antigenic form of the performance index.

The simulation is carried out using a binary representation of 14 bits to model the antibodies. The size of the initial population is 70 antibodies. The algorithm converges after the tenth generation. For simplicity the results presented here are for the gain $\mathrm{kp} 1$ of area1. Figure 3.13 presents the deployment of the antibodies after the first generation for a heuristic search of the optimal proportional gains. We notice here that the antibodies are distributed in a large interval and trying to perform a global search for the minimum of the performance index. The antibodies with low affinity, high value of $\mathrm{J}$, are considered as weak antibodies, top half of the antibodies presented on the figure, and should be highly mutated. However, the antibodies with low value of performance index, bottom half of the antibodies presented on the figure, are chosen to become memory cell. The trial method consists on substituting all the possible values of the proportional and integral gains in the system in order to validate the results.

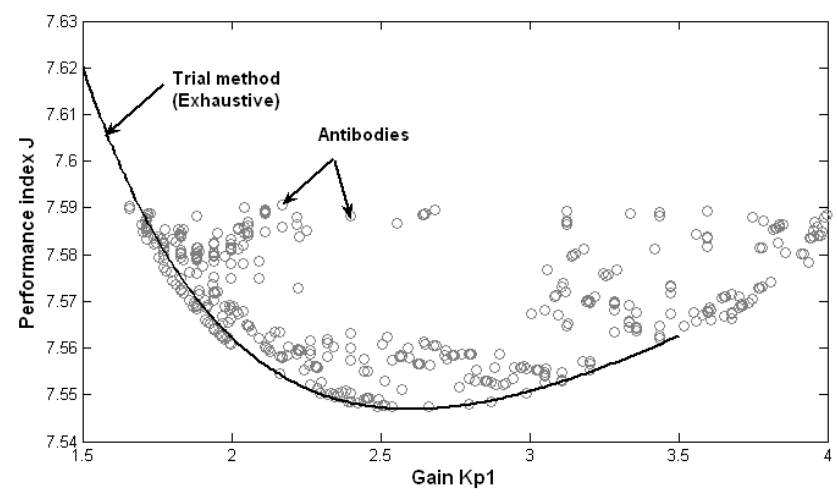

Figure 3.13 Distribution of the antibodies after the $1^{\text {st }}$ generation

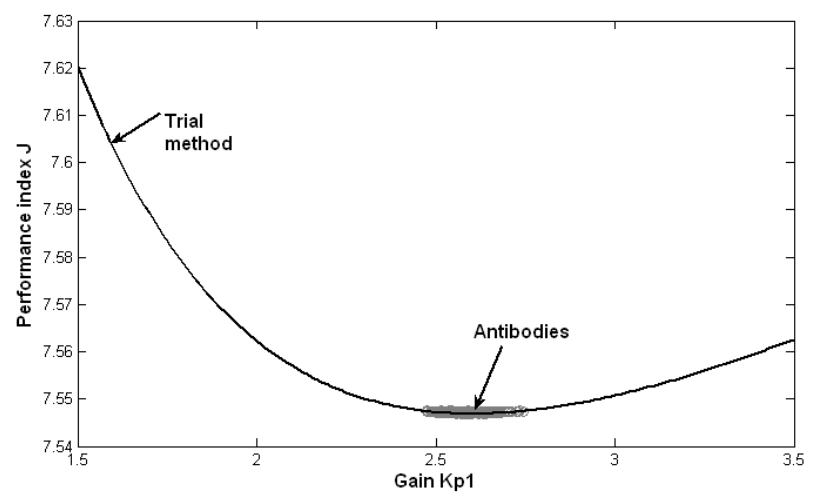

Figure 3.14 Distribution of the antibodies after the $7^{\text {th }}$ generation 


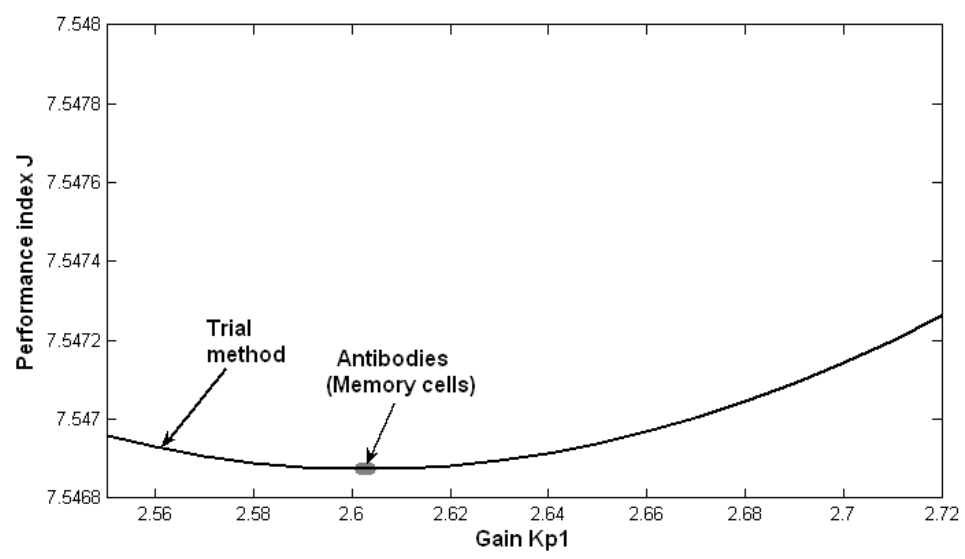

Figure 3.15 Distribution of the antibodies after the $11^{\text {th }}$ generation

In Figure 3.14 the state of the antibodies after the $7^{\text {th }}$ generation are shown, we notice that the algorithm found the global minimum and started to perform an exhaustive local search around the point $(\mathrm{kp} 1=2.6)$. After the $11^{\text {th }}$ generation, Figure 3.15, all the antibodies converge to the minimum of the performance index represented here on the y-axis.

\subsection{Immune based NERC standards oriented controller design}

In this section, an immune based controller using the immune network theory is designed to manipulate the conventional integral-type load frequency controller to achieve two objectives: (i) minimize equipments' wear and tear and (ii) comply with NERC's CPS1 and CPS2. The proposed controller is totally decentralized using just the measurements of the frequency and the Tie-line power exchange of each area, see Figure 3.16.

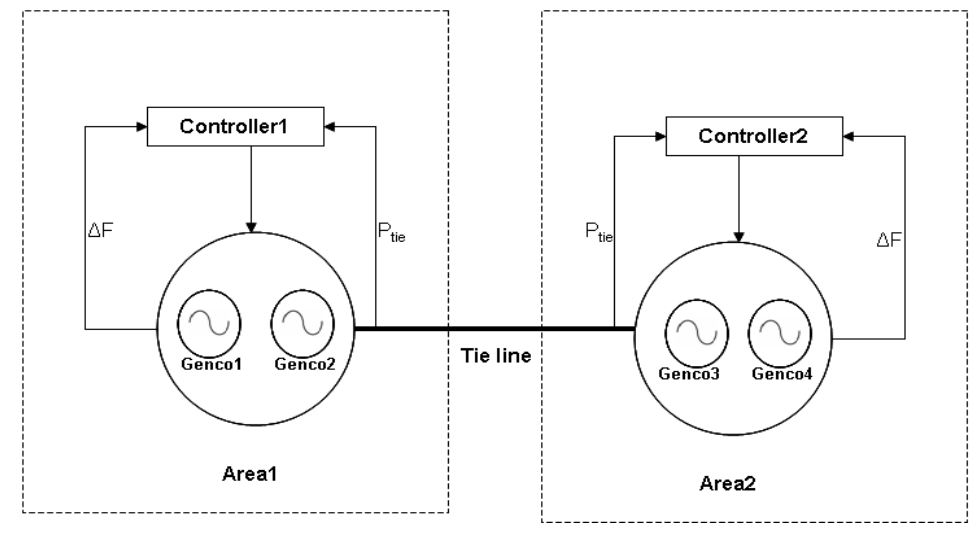

Figure 3.16 The proposed decentralized control 
The control structure for each area is of the form:

$$
u=\Delta P c=K_{I} \int A C E d t
$$

where $(\triangle P c)$ is the governor setpoint or raise/lower signal, $K_{I}$ is the integral-control parameter. The proposed control will lower the control parameter when the control area has high compliance. On the other hand, that control parameter will be increased when the compliance with CPS1 of the control area is low. this algorithm will significantly reduce wear and tear of unit equipment since movements of the governor setpoint or raise/lower signal $(\Delta \mathrm{Pc})$ generated from the integral controller are less frequent when the control area has high compliance or that values of 1-minute average compliance factor (CF1).

The first input to the controller is CF1, which is defined as the product of the area control error ACE and frequency deviation $\Delta F$. Thus poor compliance with CPS1 is indicated, when this value is high. On the other hand, a control area is in good compliance with CPS1, when this value is low. The second input is the 10 minute window average of the ACE. It is used to measure the compliance with CPS2, Figure 3.17.

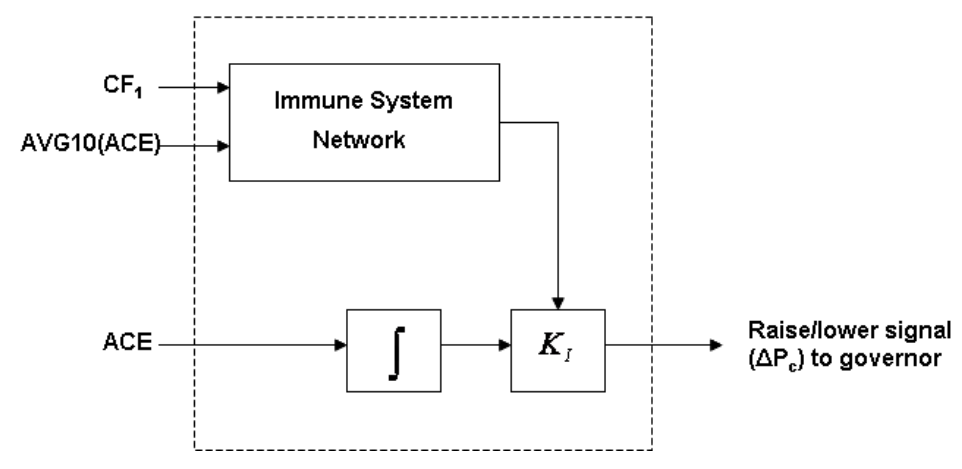

Figure 3.17 Immune -based load frequency control

\subsubsection{Designing the Immune System Network}

As mentioned earlier, the Immune network theory models the immune response in terms of a network of antibodies that stimulate and suppress each other. Antibodies that recognize either the antigen or other antibodies and lock on them, effectively suppress those antigens and antibodies. The first step of designing our controller is to define the antibodies and the antigens for LFC problem. In this study the antigens is simply represented by the 1-minute average of the ACE and the excess maneuvering of the unit since the goal of the controller is reduce the maneuvering and 
comply with the NERC standards. Next, we explain how we describe the antibody. As mentioned previously, the identity of each antibody is generally determined by the structure or molecular shape of its paratope and idiotope. We assign a pair of precondition and behavior to the paratope and the ID-number of the stimulating antibody and the degree of stimuli to the idiotope, respectively. The structure of the precondition is same as the antigen described above. We prepare the following behaviors:

- Increase the parameters

- Decrease the parameters

- Keep the parameters constant

For the antibody selection, an index that quantitatively represents suitableness under the current situation is necessary. We assign one variable called concentration of antibody to each antibody. This variable represents the actual level of stimulation and suppression for that antibody in the network. Antibody with maximum concentration is selected, in a "winner-takes-all" fashion. For the $i^{\text {th }}$ antibody, stimulus and concentration are calculated by the following equations (Ishiguro, 1995).

$$
\begin{aligned}
& \frac{d S_{i}(t)}{d t}=\left(\alpha \sum_{j=1}^{N} m_{i j} s_{j}(t)-\alpha \sum_{k=1}^{N} m_{i k} s_{k}(t)+\beta g_{i}\right) s_{i}(t) \\
& s_{i}(t+1)=\frac{1}{1+\exp \left(0.5-S_{i}(t)\right)}
\end{aligned}
$$

where, $i, j=0,1, \ldots, N-1$, and $\alpha, \beta$ are constants,

$\mathrm{N}=$ number of antibody types,

$\mathrm{S}_{\mathrm{i}}(\mathrm{t})=$ Stimulus of antibody $\mathrm{i}$,

$\mathrm{s}_{\mathrm{i}}(\mathrm{t})=$ Concentration of antibody $\mathrm{i}$,

$\mathrm{s}_{\mathrm{j}}(\mathrm{t})=$ Concentration of antibody $\mathrm{j}$ for other antibodies,

$\mathrm{m}_{\mathrm{ij}}=$ Mutual stimulus coefficient between antibody $\mathrm{i}$ and $\mathrm{j}$,

$\mathrm{g}_{\mathrm{i}}=$ affinity of antibody $\mathrm{i}$ towards antigen.

In Figure 3.18, we show the antibody network for the NERC oriented control design. 


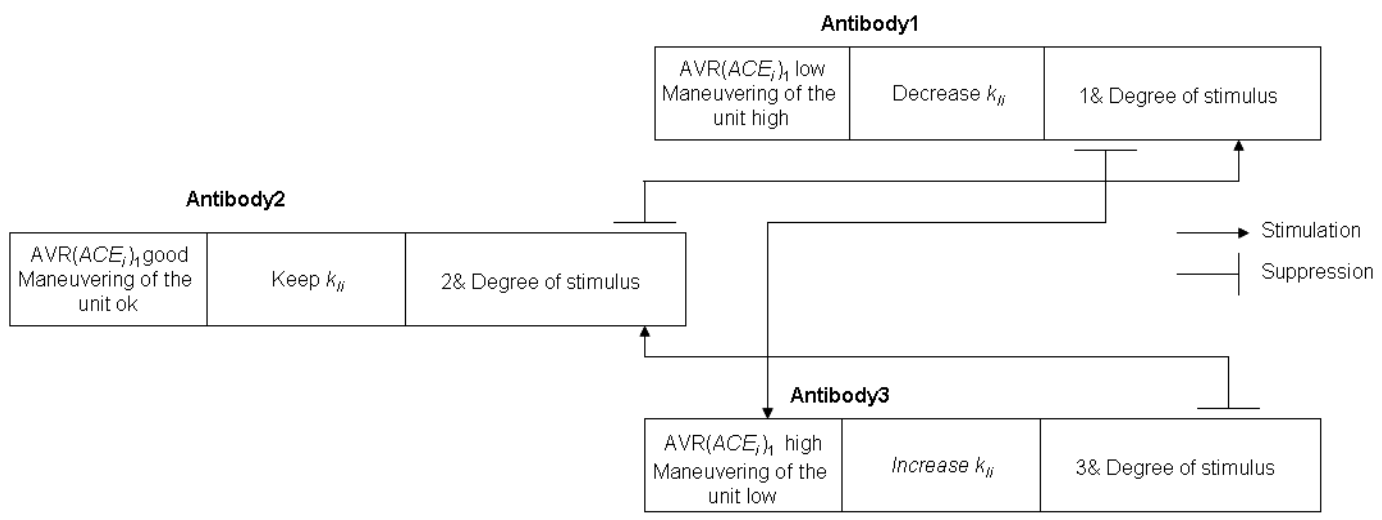

Figure 3.18 Antibody networks for the LFC problem

\subsubsection{Simulation results}

To demonstrate the performance of the proposed strategy a two area power system as shown in Figure 12 is chosen as a test system. It includes 2 generation units in each area. The proposed load frequency control of each area is used to satisfy power demand during a load pick-up hour as shown in Figure 3.19.

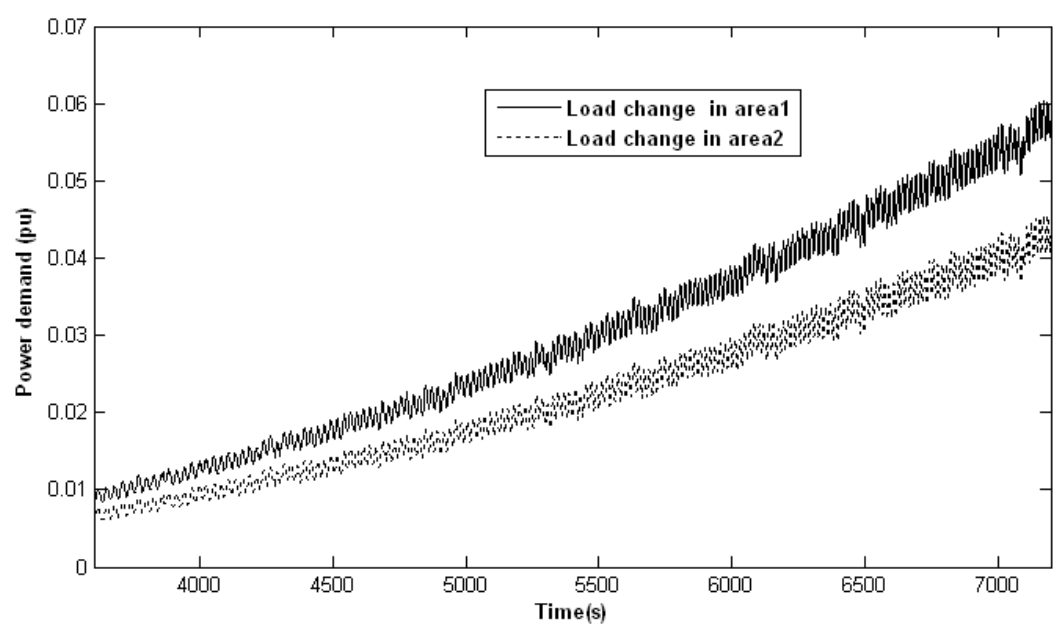

Figure 3.19 load demand in each area

As follows the performance of the immune based control is assessed through nonlinear simulation. Its performance is compared to conventional control designs: a conventional design with existing control parameters, which are currently used by control areas, identified as Case1, and another conventional design using much lower control parameters identified as Case 3 . The immune based control is identified as Case2. the reason for choosing these three cases for 
assessing the proposed load frequency controller are to show that 1) IS based controller can reduce unnecessary wear and tear by lowering the values of the original control parameters and also 2) these new adjustable control parameters designed are capable of helping the control areas comply with CPS2. Case3 shows how using control parameters that are two low causes the control areas to violate CPS2, although wear and tear is reduced.

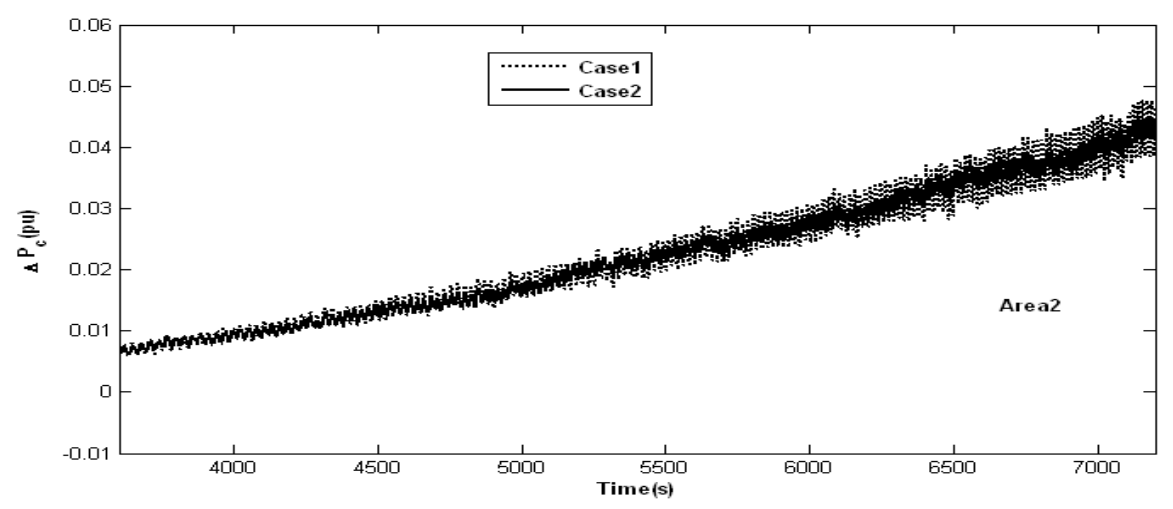

Figure 3.20 Governor load set point for area1

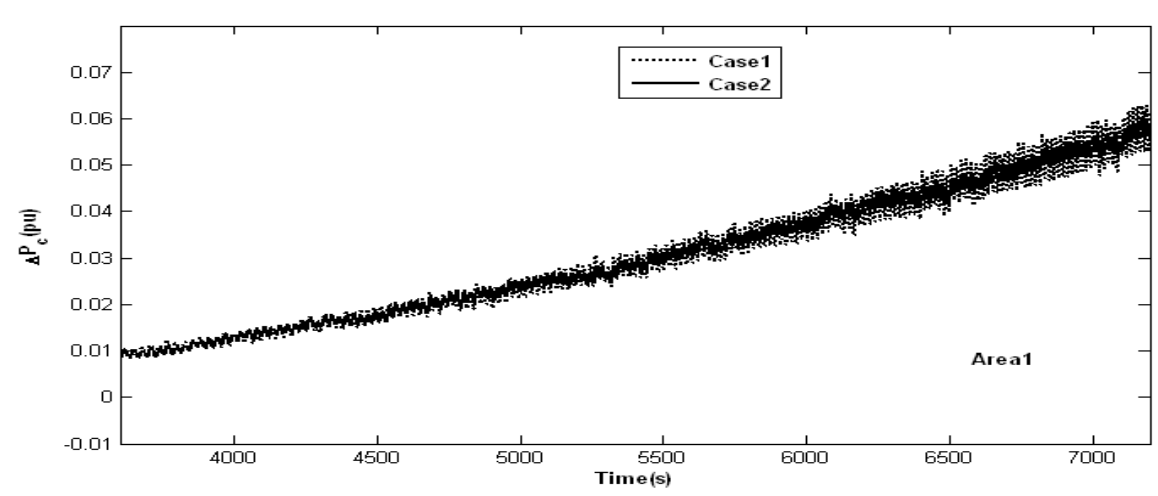

Figure 3.21 Governor load set point for area2

Figure 3.20 and Figure 3.21 show the plots of changes in the load set point or rise /lower signals $\Delta \mathrm{P}_{\mathrm{c}}$ of each generating unit for Case 1 and Case 2. Case 2 and 3 are similar since in both cases the parameters are kept low. We notice that the signals from Case 2 have fewer high frequency components than those of Case1.The superior performance of the Immune based control is better 
seen in Figure 3.22 and Figure 3.23. The latter reduces unit maneuvering and high frequency components.

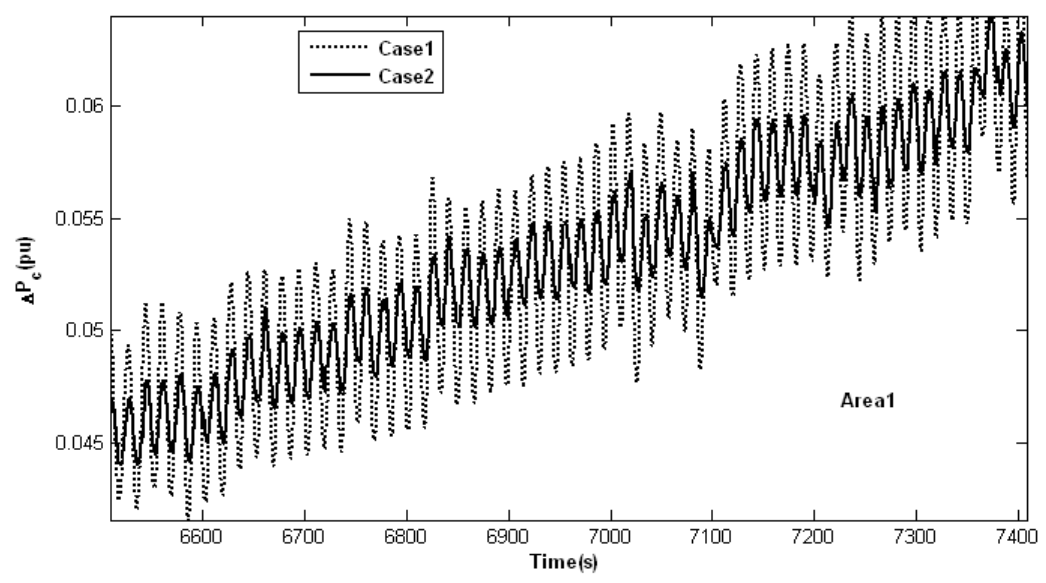

Figure 3.22 Zoom on governor load setpoint curve for area1

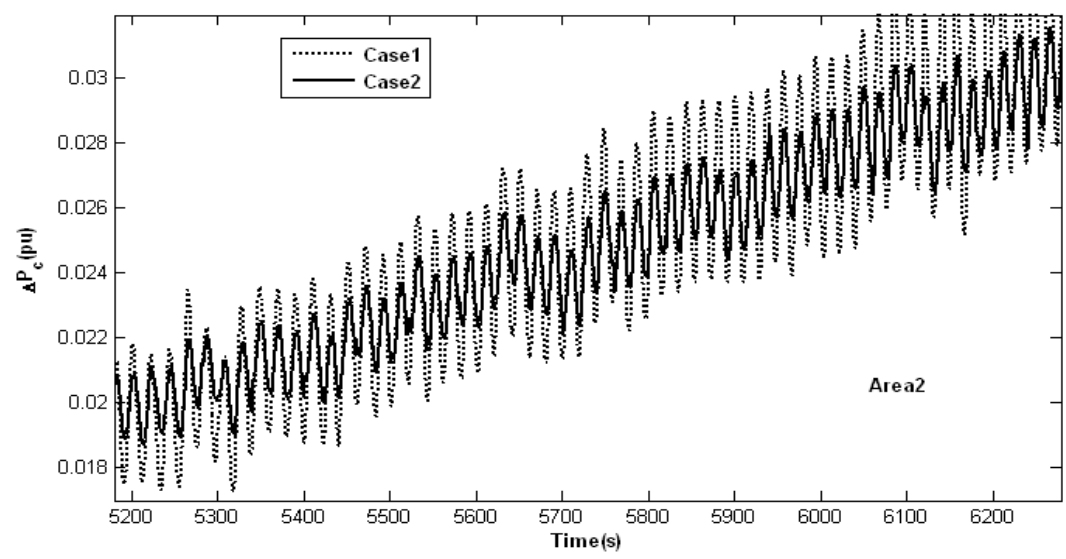

Figure 3.23 Zoom on governor load setpoint curve for area2

The control parameters are plotted in Figure 3.24. The control parameters are automatically tuned to reduce wear and tear, and help the control area compliant with NERC standards, whereas those for the other cases are fixed, and not as versatile as the Immune based one. In the case study, all areas are highly compliant with CPS1 but the proposed controller automatically lowers the level 
of this compliance to gain benefits the reduction of wear and tear. The percentage of compliance with CPS1 for each control is plotted in Figure 3.25 and Figure 3.26.

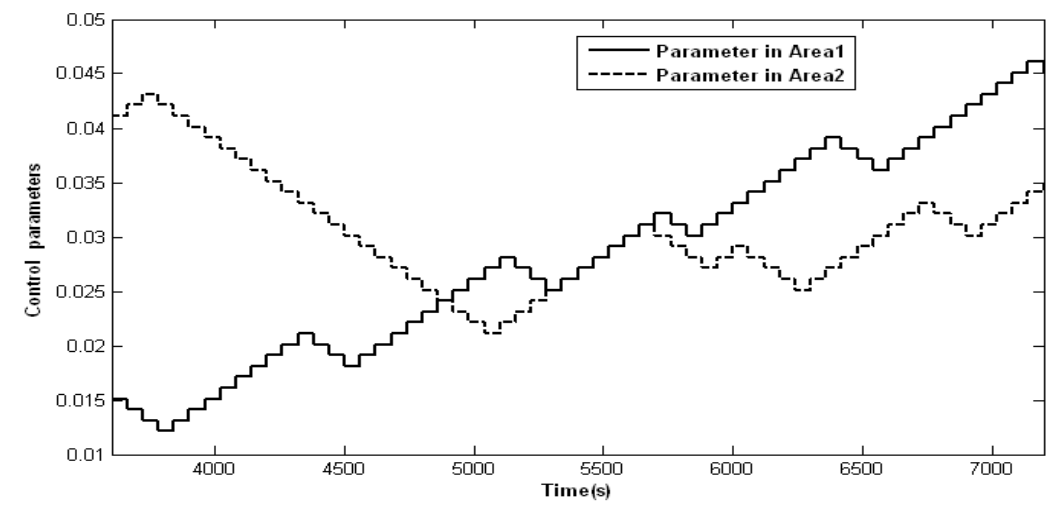

Figure 3.24 Control parameters variation

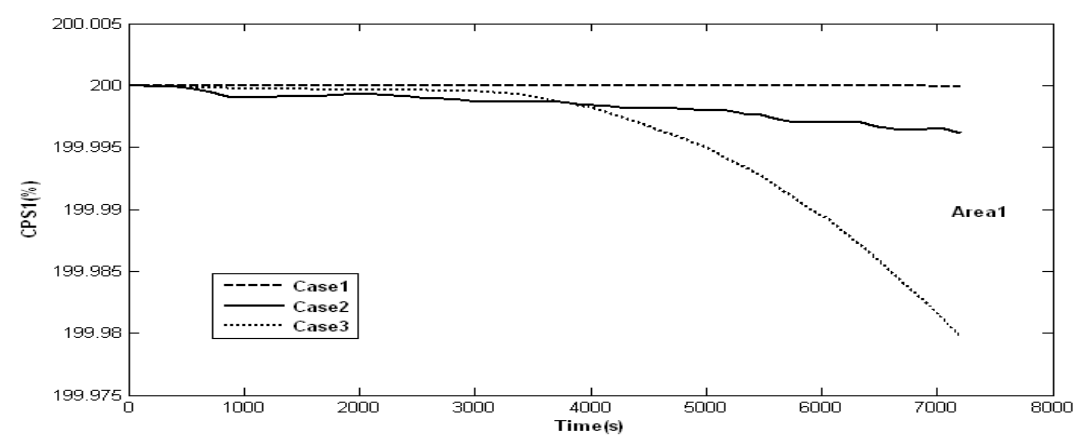

Figure 3.25 Percentage of compliance with CPS1 for area 


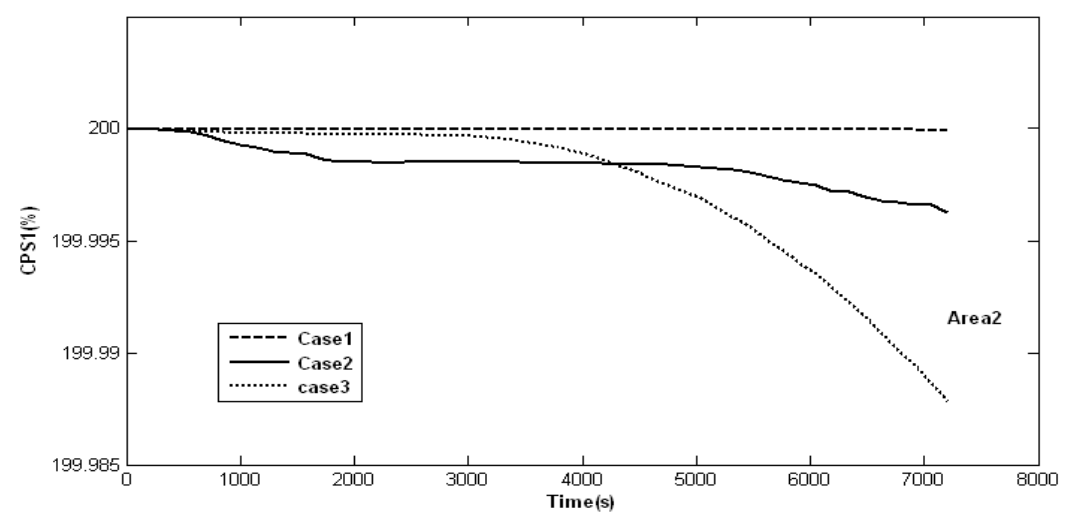

Figure 3.26 Percentage of compliance with CPS1 for area2

In Figure 3.27 and Figure 3.28, the 10-minute averages of the ace of control areas are computed and compared with the constants $\mathrm{L}_{10}$. The control parameters from Cases 1 and 2 successfully help the areas comply with CPS2, but those for Case3(low control parameters) could not keep the 10minute averages of the ACE in both areas within the limit during the period 7:40-8:00 PM for Area1 and 7:20-8:00 PM for Area2 Table3.1.

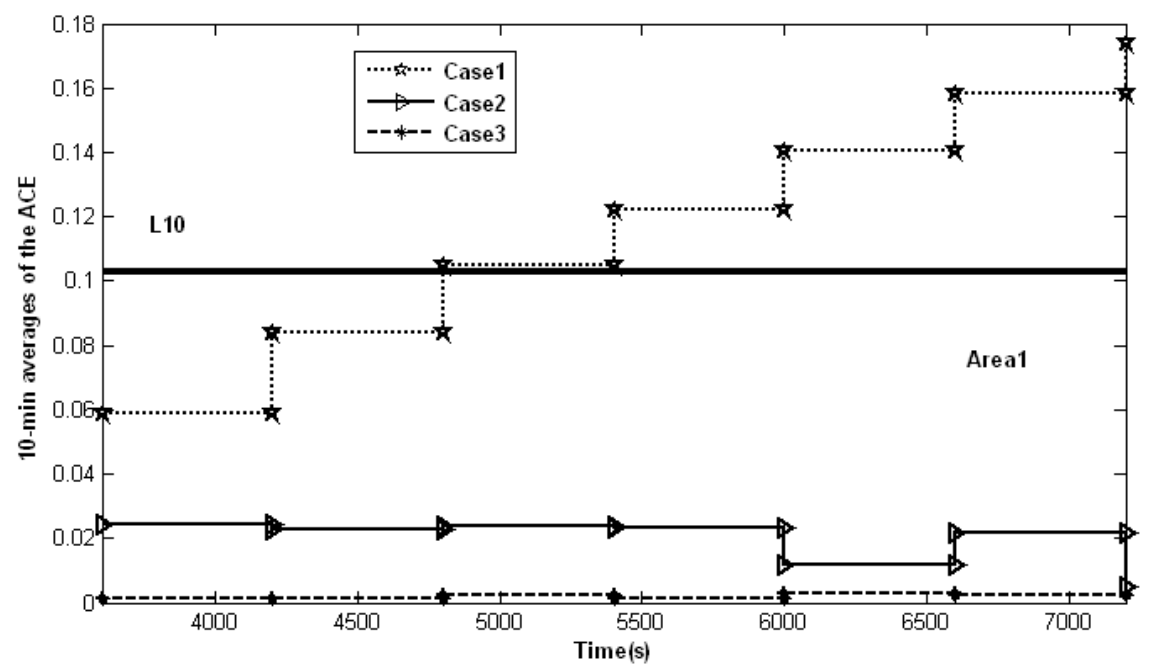

Figure 3.2710-minute average of the ACE for area 1 
Chapter 4 A Decentralized Immune Based Control Design of an Automatic Generation Control

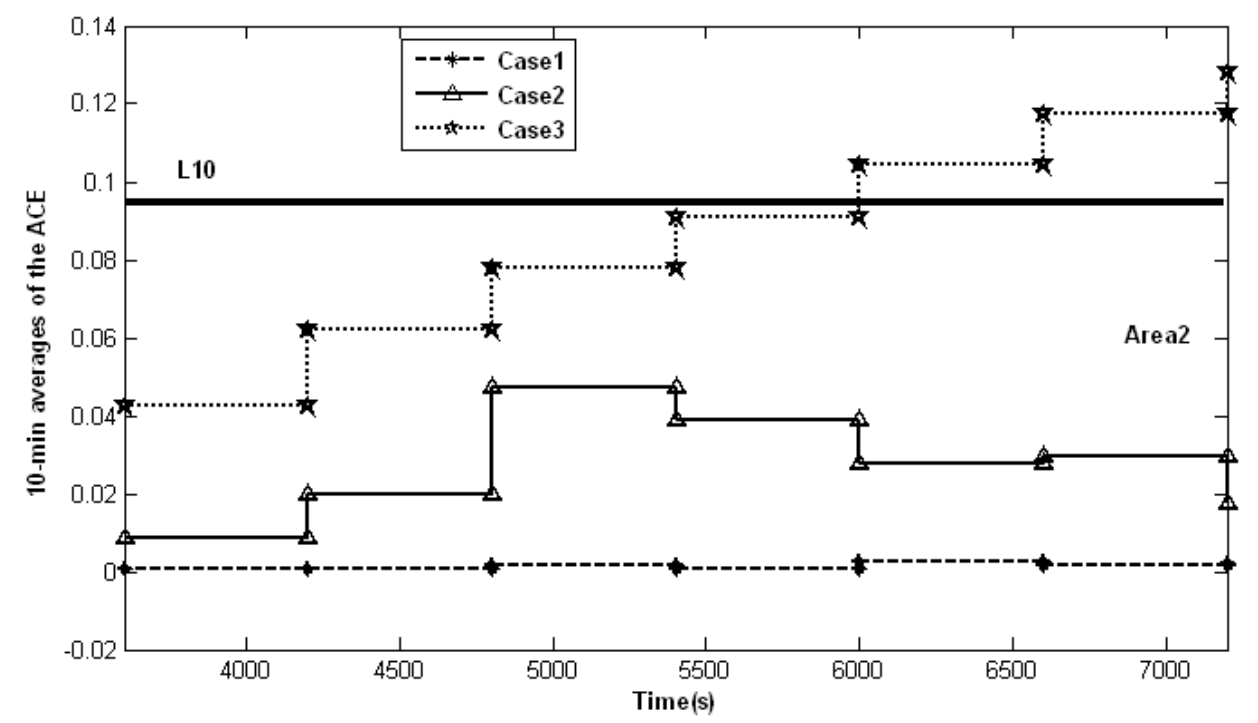

Figure 3.2810-minute average of the ACE for area 1

Table 3.1 10-minute Average of the ACE

\begin{tabular}{lcccccc}
\hline & \multicolumn{3}{c}{ Area1 } & \multicolumn{3}{c}{ Area2 } \\
Time & Case1 & Case2 & Case3 & Case1 & Case2 Case3 \\
\hline 7:00-7:10 & 0.0012 & 0.0245 & 0.0586 & 0.0009 & 0.0087 & 0.0426 \\
$7: 10-7: 20$ & 0.0012 & 0.0227 & 0.0839 & 0.0009 & 0.0199 & 0.0619 \\
$7: 20-7: 30$ & 0.0022 & 0.0236 & 0.1048 & 0.0018 & 0.0472 & 0.0778 \\
$7: 30-7: 40$ & 0.0014 & 0.0235 & 0.1223 & 0.0009 & 0.0389 & 0.0907 \\
$7: 40-7: 50$ & 0.0031 & 0.0115 & 0.1405 & 0.0024 & 0.0279 & 0.1045 \\
$7: 50-8: 00$ & 0.0022 & 0.0216 & 0.1583 & 0.0015 & 0.0297 & 0.1173 \\
& & & & & & \\
\hline
\end{tabular}

Finally, simulation results show that excess maneuvering, which normally exists for the conventional LFC, is diminished by the proposed controllers. In addition, the control parameters of the IS-based are effective in helping the control areas comply with CPS. 


\subsection{Conclusion}

The biological immune system is a complex and effective control mechanism that continuously monitors and protects the human body very effectively. It learns, adapts and attacks and destroys viruses and bacteria that it had or did not have encountered in the past. This amazing system, if mimicked correctly could have an impact on how to protect complex engineering systems. This is the main objective of the work presented here, that deals with intelligent control of electric power systems.

The human immune system is a complex system of cells, molecules and organs with the primary function of protecting the body from attacks by eliciting an immune response. The attack in this case is by pathogens or infectious agents, such as bacteria and viruses. The immune system mimics a control strategy in an electric power system setting which operate around the clock to deliver electricity to its customers. In the framework of the approach presented in this work, the power system, with its protection scheme and distributed control structure, is analogous to a living human body. Anomalies or disturbances in the system, and deviations from normal operating conditions, are then seen as pathogens or damages that the system strives to eliminate. Using this concept of immune cells-pathogens the power system control design is addressed in general, and in particular the automatic generation control problem is used to illustrate this approach and demonstrate its feasibility. In this chapter, first a brief description of the biological immune system is presented and explained in a war-like setting, and simple examples in power system terminology and optimization are given. Second, the automatic generation control design problem is cast as an immune based optimization problem. A two-area power system is used to demonstrate the effectiveness of this novel approach.

First, the Clonal Selection theory is successfully applied as an optimization technique to obtain decentralized control gains that minimize a performance index based on Area Control Errors. Second, the Immune Network theory is used to design adaptive controllers in order to diminish the excess maneuvering of the units and help the control areas comply with NERC's standards set to insure good quality of service and equitable mutual assistance by the interconnected energy balancing areas. The results show the effectiveness of the immune based controllers over conventional controllers in the case of two area system.

In summary, intelligent techniques can improve the performance of power systems operation and make it a flexible and autonomous system that could reduce vulnerability of the power grid. 


\section{Chapter 4}

\section{IMMUNE BASED MULTI-AGENT SYSTEM}

This chapter presents a new algorithm for fault detection, reconfiguration, and restoration of the power distribution systems based on the Immune Multi-Agent System (IMAS). The theory about the design is presented first followed by the application on the Southern California Edison Circuit of the Future and then on the Shipboard power system hardware test bed.

\subsection{Multi-Agent System}

In this section, a brief definition of Multi-Agent System (MAS) is given as well as a reason for applying it to power systems. Weiss [50] defines an agent as "an autonomous computational entity such as software program that can be viewed as perceiving its environment through sensors and acting upon this environment through its effectors". So, a multi-agent system is composed of several of this kind of agents interacting with each other to achieve a global goal which is beyond their individual capabilities. Hence, this type of technology can allow us to distribute and localize the control of power systems. By incorporating intelligence at the device level the reliability of the system should improve dramatically since there is no single point of failure as compared to the centralized control.

MAS can be depicted as shown in Figure 4.1 below. 


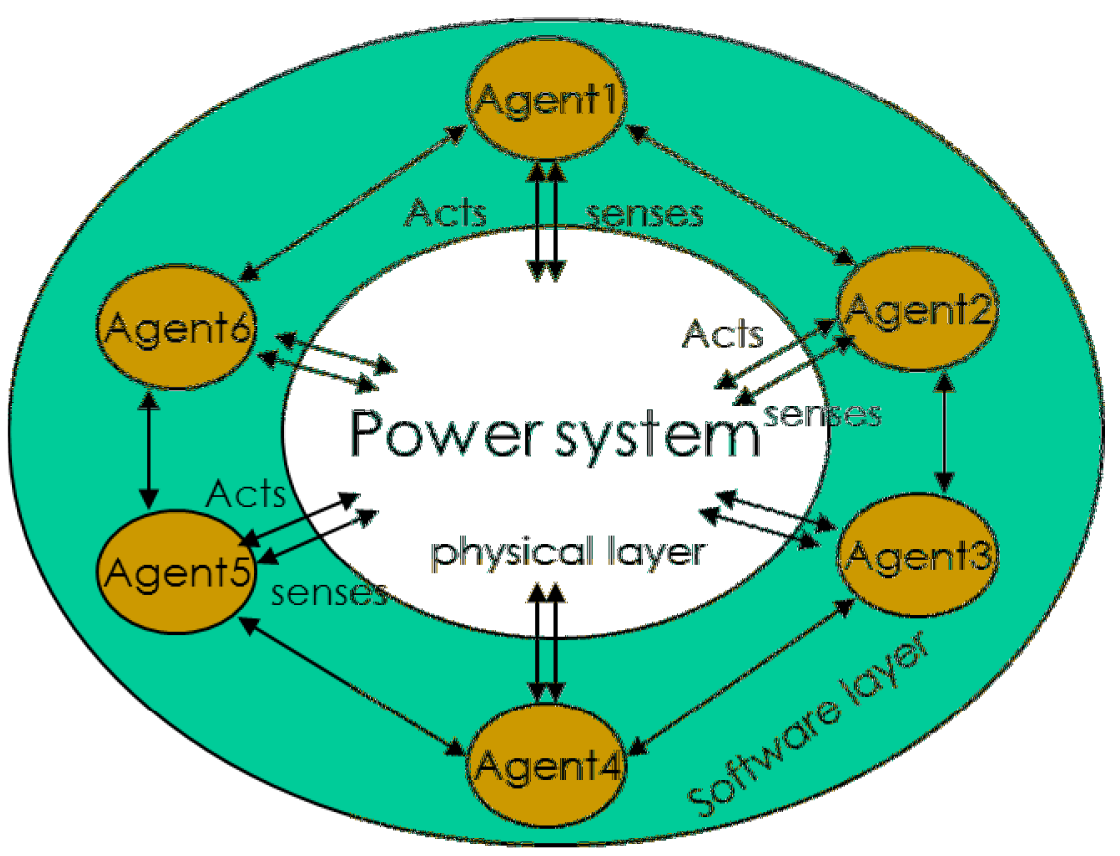

Figure 4.1 Multi-agent System in the sense of Power System

In addition, an agent has to be autonomous, which means that an agent can operate without direct human intervention. It can decide on its own, the required actions to be taken in order to achieve its objectives. Therefore, for MAS to be entirely autonomous, it has to have the ability of being an intelligent system. MAS without intelligence is no different to another software application, where the decision making process is designed by the programmer and if any unexpected situation other than a planned event occur, the system would crash. Hence, intelligent agents have to encompass the following characteristics [51].

- Reactivity: Intelligent agents should have the ability to perceive the environment in which they live and respond in a timely manner to any changes or disturbances that take place in the environment in order to achieve their own goals

- Proactiveness: Intelligent agents should have the ability to take initiatives on their own in order to meet their goal directed behaviors 
- Social ability : Intelligent agents should have the ability to interact with other agents in the system by coordinating, communicating and negotiating by using a standardized agent communication language

\subsection{Agent Communication}

It is important to have a common Language for Agent Communication, without which, the coordination and negotiation will hardly be successful.

The communication protocol must be universally shared by all agents. It should be concise and have only a limited number of primitive communication acts. The most popular Languages followed for Agent Communication are,

- Agent Communication Language (ACL) by the Foundation for Intelligent Physical Agents (FIPA)

- Knowledge Query and Manipulation Language (KQML)

For the agents to understand each other, they should not only speak the same language but also have to follow the same ontology. Two agents, while communicating with each other, need to agree on the same terminology. For example, in a power system, when an agent is requested to by another to perform Current Measurement "IM", it has to know what IM means.

\subsubsection{FIPA - ACL}

In our work we are using the FIPA protocol. FIPA is an organization to develop software standards for heterogeneous and interacting agents and agent based systems.

A FIPA ACL message will contain one or more message parameters, according to the requirements of the agent application. There is one essential parameter that has to be specified in all the agent communication, which is the 'performative' parameter. In addition to this parameter, other important ones for effective communication are the 'sender', 'receiver' and the 'content' parameters. FIPA ACL also allows the usage of user-defined parameters other than the ones which are specified by FIPA, showed in Table4.1 below. However, a prefatory string "X-“ must be used for the names of the user-defined message parameters which do not come under standard FIPA parameters. 
Table 4.1 FIPA ACL Message Parameters

\begin{tabular}{|l|l|}
\hline \multicolumn{1}{|c|}{ Parameter } & \multicolumn{1}{c|}{ Category of Parameters } \\
\hline performative & Type of communicative acts \\
\hline sender & Participant in communication \\
\hline receiver & Participant in communication \\
\hline reply-to & Participant in communication \\
\hline content & Content of message \\
\hline language & Description of Content \\
\hline encoding & Description of Content \\
\hline ontology & Description of Content \\
\hline protocol & Control of conversation \\
\hline conversation-id & Control of conversation \\
\hline reply-with & Control of conversation \\
\hline in-reply-to & Control of conversation \\
\hline reply-by & Control of conversation \\
\hline
\end{tabular}




\subsection{Immune Based Multi-Agent System}

In this section, a MAS as defined above is designed using an Immune System approach. By observing natural systems such as Ant colonies, bee colonies or Human Immune Cells System, we realize that many of them act or form a natural Multi Agent System. The Immune System is a highly organized, distributed and intelligent Multi Agent System which is composed of millions of cells acting as an independent agent. These agents work together and communicate to mount global strategies against any bacteria or virus that invade the human body. In the following we shall give an overview on how the Immune System behaves as an intelligent MAS in the case of a body invasion by a bacterial substance, Figure 4.2 .

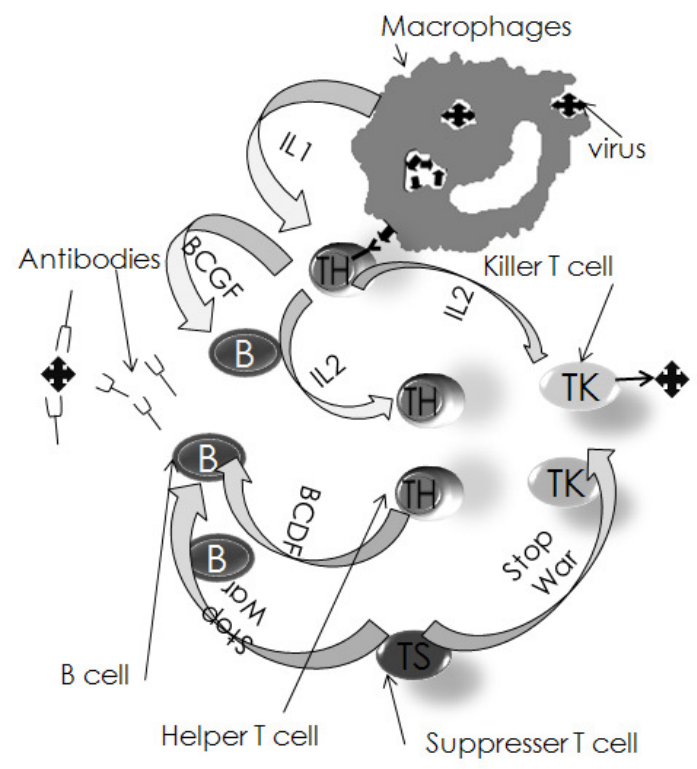

Figure 4.2 Immune Multi Agent System

First, the Macrophage (eater cell) takes in and ingests the invader organism (Bacteria) and binds to a helper $\mathrm{T}$ cell for antigen presentation. The secretion that activates the helper $\mathrm{T}$ cell (interleukin, IL-1) also stimulates the brain to increase the body temperature. This causes the common cold, which in turn increases the activity of immunity cells.

Once activated, the Helper T cell produces interleukin 2 (IL2) which causes the other helper T cell, B-cells and killer-T cells to develop and divide (BCGF-B Cell Growth Factor). 
When the number of B cells increases, helper T cells produce another substance, which orders B cells to stop multiplying and start producing antibodies (BCDF-B Cell Development Factor). With the same signal, helper $\mathrm{T}$ cells also activate killer $\mathrm{T}$ cells.

After the bacterial invasion is neutralized Suppressor T cell to the other cells to stop the attack. Memory $\mathrm{T}$ and $\mathrm{B}$ cells remain in the blood and the lymphatic system in order to become immediately activated in case a bacterial substance of the same type invades the body again.

Now we have seen how the immune system operates as MAS with its entire characteristics, we shall try to design a MAS that operates on a power systems based on the behaviors explained above.

In the case of the power system, the bacteria or virus is analogous to faults on the grid such as short circuit faults, overloading of the feeders, voltage violations, power generation shortage, etc. The operation performed by the Macrophages of ingesting the bacteria and presenting it to the others cells is incorporated as an Agent behavior MacrBehavior in the body of each Agent to be designed by which an agent accesses information about abnormalities or violations in the current or voltage through the sensors or through the inter agent communication, Figure 4.3. One of the requirements set by Allegheny Power (AP) in their Super Circuit is that no action can be taken by the agents after a fault in the system unless a lock out signal from the recloser is received. In this case the Macrophage behavior consists in the fault location by the agents using the prefault data. Once the fault is located, the faulted area is isolated first then the information is shared with the agents that can be involved in the reconfiguration restoration process.

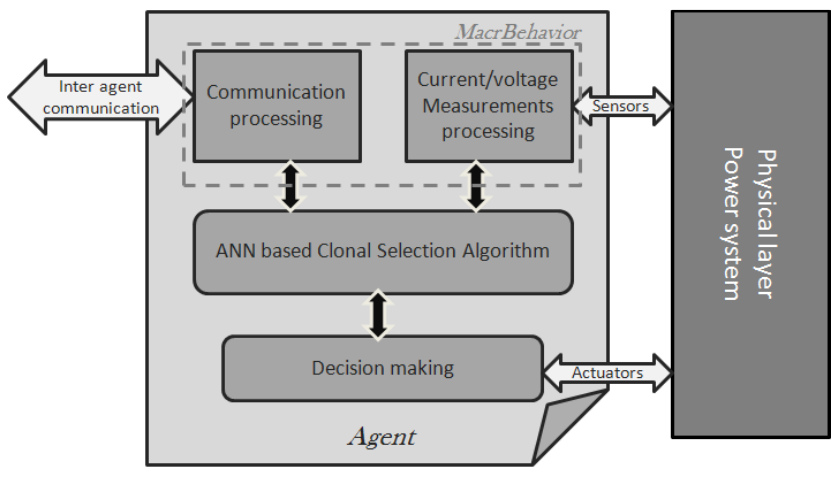

Figure 4.3 Single Agent Architecture

The second block in the agent body is designed by mimicking the thymus organ in the Human Immune System. This organ is responsible for training and teaching new born cells about the 
body and how to deal with foreign substances. In designing our agent we shall use the Clonal Selection Theory in order to perform the training process.

This theory proposes that as antigen (bacteria) enters the body certain cells are selected based on their reaction to this antigen to undergo rapid cloning and expansion. Those cells with a sufficient affinity are allowed to produce offspring in relation to their degree of stimulus. Some cells transform into plasma cells and others are selected to become long lived memory cells which allow for a more rapid secondary response to the same or similar antigen. The training process is then incorporated as a Neural Network Block in the agent body for it to be used as a brain to help make decisions.

In Table 4.2, we try to map and extract analogies between cells and behaviors in the Immune System compared to agents and processes for power system control. 
Table 4.2 Power System Immune System MAS analogy

Biological System
Thymus training
The thymus is an organ located in the upper
anterior portion of the chest cavity which is
responsible for training and teaching the newborn
cells and has important information and
characteristics of the body and its cells.

\section{B-cells}

These cells are responsible for producing antibodies in case of an invasion of the body by harmful substances. The cells or the antibodies perform Clonal Selection to recognize the substances and destroy them.

\section{Killer T-cells}

These cells are usually activated by a signal from the Helper T-cells. They are equipped with a special molecule that enables them to recognize the foreign substances and the infected body cells without performing any mutation or Clonal Selection.

\section{Training Process}

This behavior is mimicked by using the Clonal Selection Algorithm to train certain types of agents before they are deployed on the power system. This training will constitute the brain of the agent that helps it in taking decisions.

Bus/Zone Agents
These agents are responsible for locating and
isolating faults that occur between the buses
then use the Clonal Selection Block to identify a
solution for the problem. The potential solution
is applied by means of communication and
negotiation with the different agents involved in
the process of applying the particular solution
such as SwitchAgents or HelperAgents.

\section{SwitchAgents}

These agents are located at switches and they are responsible for altering the configuration of the power network. They are activated by the HelperAgents in case of a request or a need to change the network topology.

\section{LoadAgents}

These types of agents are responsible for controlling the loads.

\section{SourceAgents}

These type of agents are located at the sources whether substations or distributed generators. They are responsible for controlling the power generation.

HelperAgents
These agents play a role of coordinator or
mediator by helping other types of agents
reaching their objectives. Some of the functions
of these types of agents are:
- Solving communication conflict
between the agents
- Creating communication bridges for
distant agents
- Checking limit violations
Distributed processing and analysis of
data
Help in reducing the complexity of the
other types of agents

4.4 Implementation on SCE's Circuit of the future Model 
The technique presented above is implemented on a model of a Southern California Edison (SCE) distribution feeder known as the Circuit of the Future. The project is designed to test new hardware and automation technologies for circuit construction. The system consists of 66 buses, one substation, 2 distributed generators and 14 residential loads with some capacitor banks scattered throughout the grid as shown in Figure 4.4. The system is divided into 3 zones connected via controllable switches. In case of faults, these switches are operated to change or reconfigure the system in order to feed the loads and restore the power in the isolated areas.

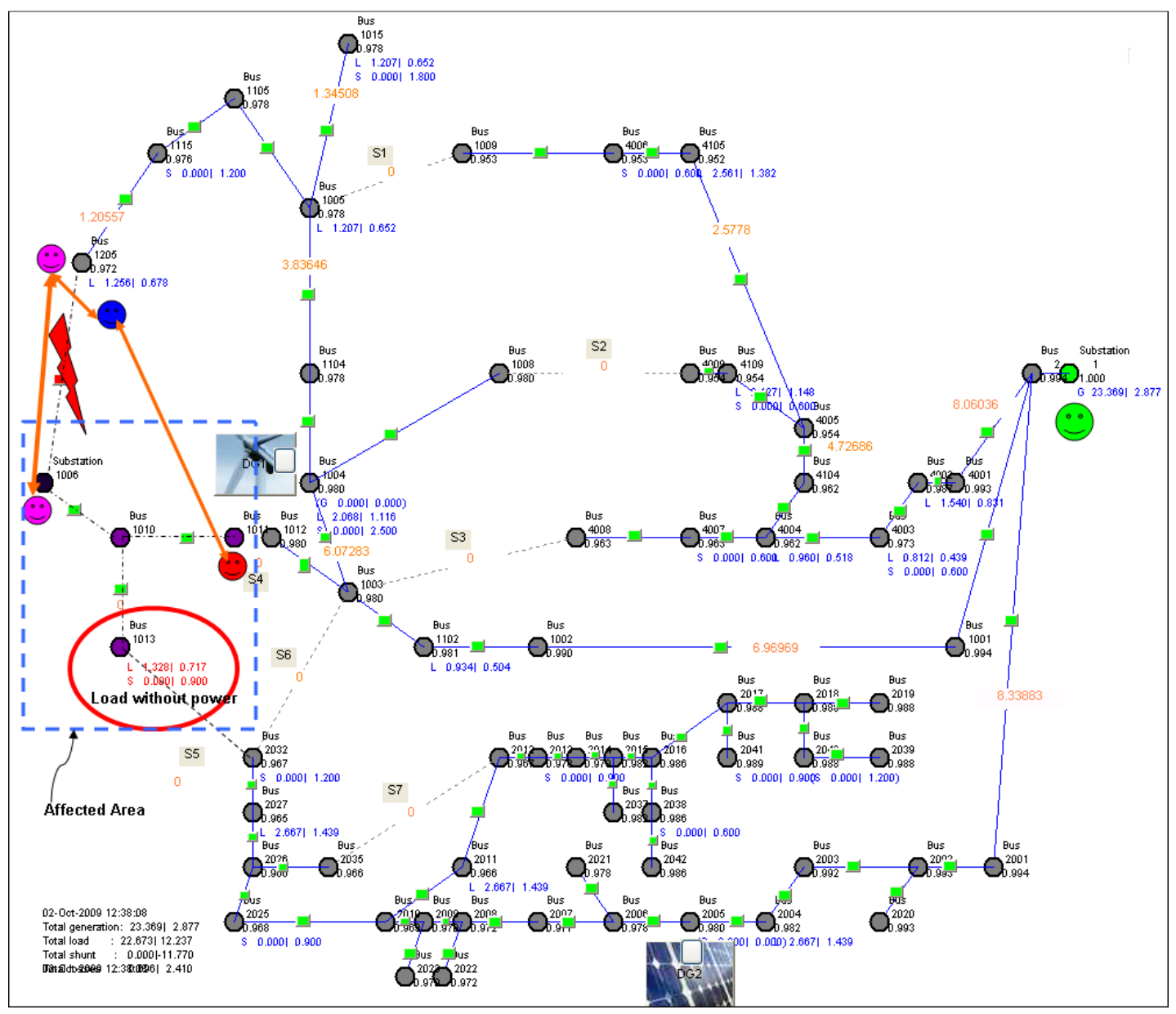

Figure 4.4 Southern California Edison Distribution System

In the following, a line fault is simulated between node number 1205 and 1005 as shown in Figure 4.4. As soon as the fault occurs, AgentNodes seating on node 1205 and 1005 start communicating the current measured and decide to isolate the line. We can see from the figure 
the region which has been affected by the fault and the load at node number 1013 has lost power. Then, these agents report the location of the fault to the BusAgent localized in that area of the circuit and a reconfiguration strategy is applied by collaboration with the SwitchAgent\#4 to restore the power to the load at node number 1013. Figure 4.5 shows the agents communication in the (JADE) Java Agent DEvelopment platform.

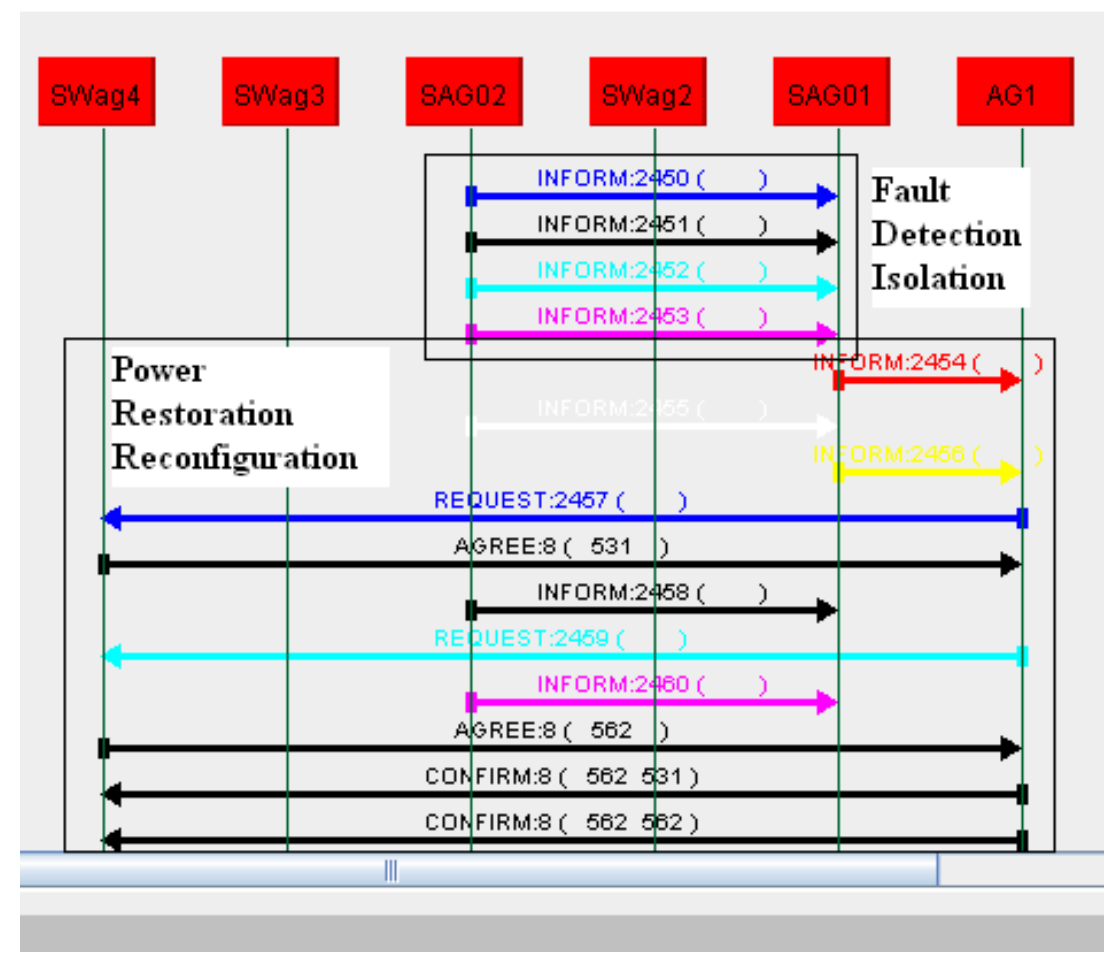

Figure 4.5 Agent communication following a fault in the system

In the Figure 4.6 below, we show the final solution and the new target reconfiguration. 


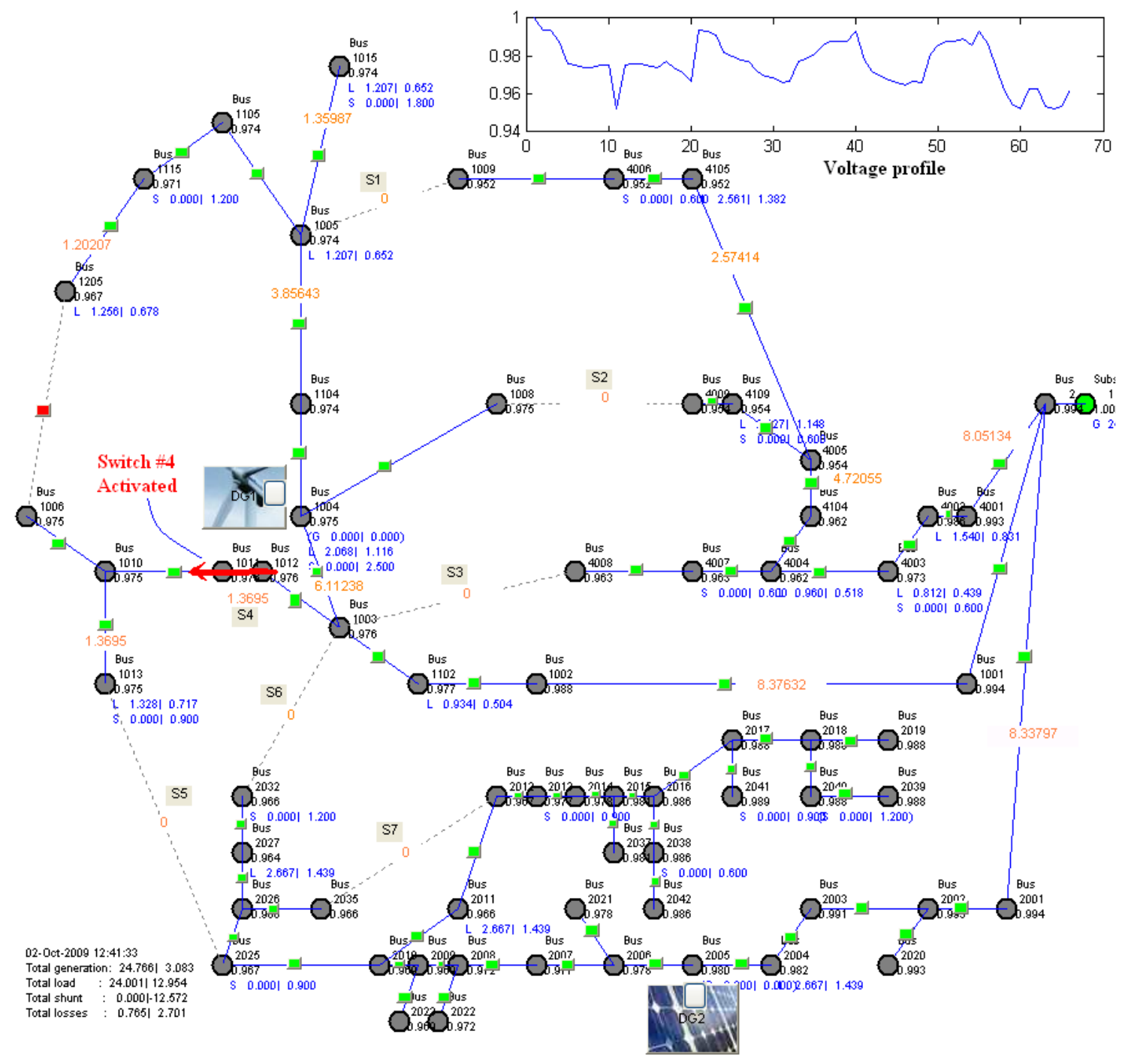

Figure 4.6 System after agents reconfiguration

The phase A current at the fault location is shown in Figure 4.7. We notice the sudden increase in the current due to the fault. Figure 4.8 shows the phase current seen at node \#1005. We notice that the fault is not seen at this location since the power is flowing form node 1205 to node 1005 . The phase current of the load at node \#1013 is shown in Figure 4.9. We notice that load lost power when the agent isolated the line, then fed after the agents performed the reconfiguration. 


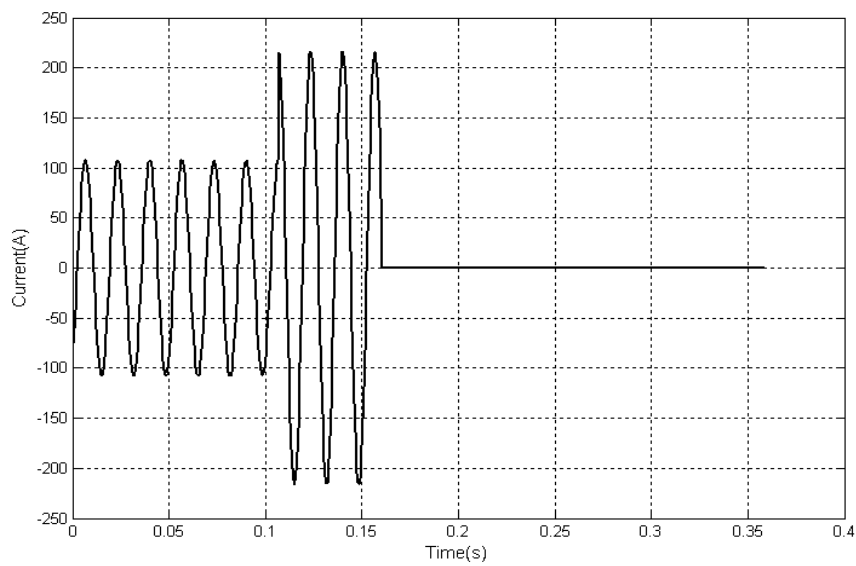

Figure 4.7 Phase A current at node\#1205

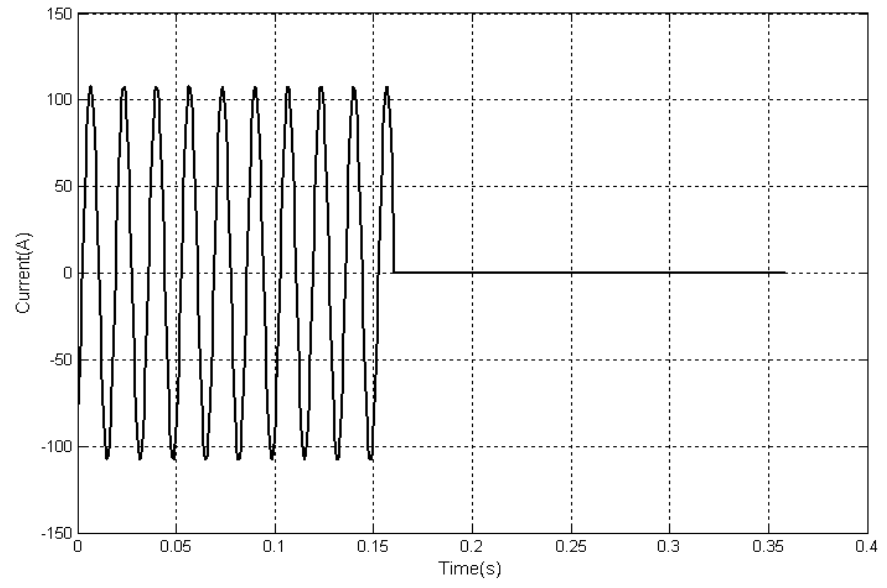

Figure 4.8 Phase A current at node\#1005 


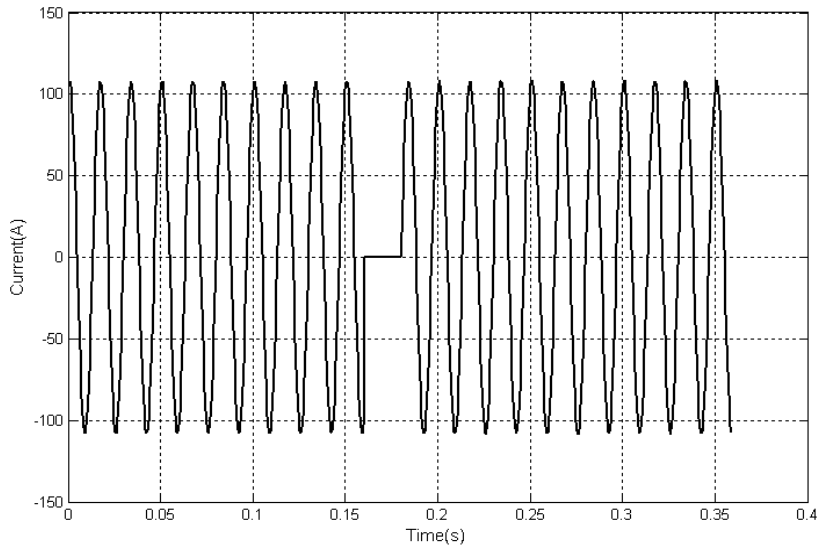

Figure 4.9 Current at Load\#1013 
In the following a line fault is simulated between bus number 1102 and 1002, Figure 4.10. We can see from the figure the region which has been affected by the fault. The objective of the algorithm is to reroute the power or find the best reconfiguration to feed the faulted area. The algorithm takes into account in searching for the suitable switching the following constraints:

- Maximum loads

- Voltage constraints

- Minimum losses

- Capacity of the lines

- Minimum switching

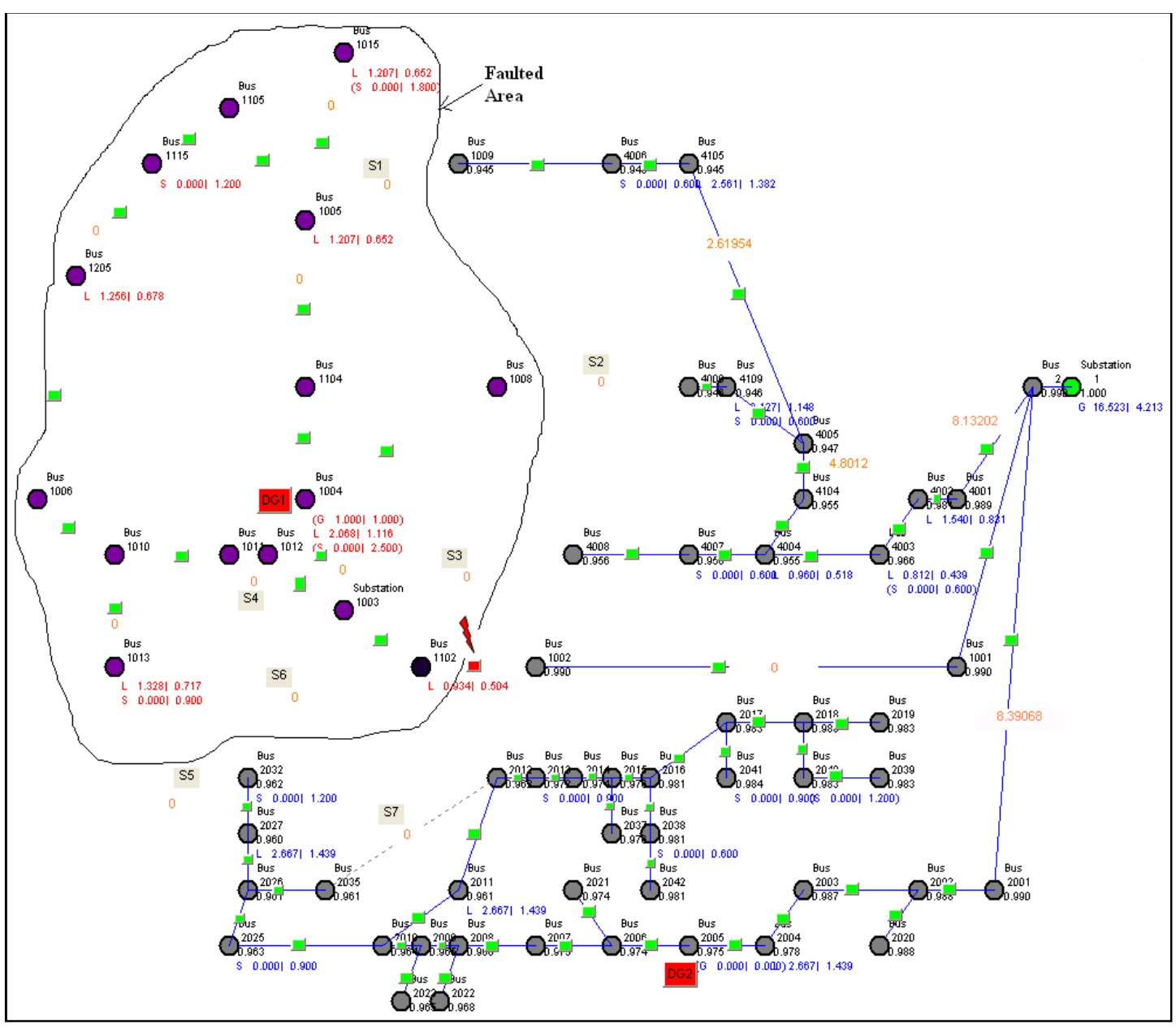

Figure 4.10 Fault at bus number 1102 


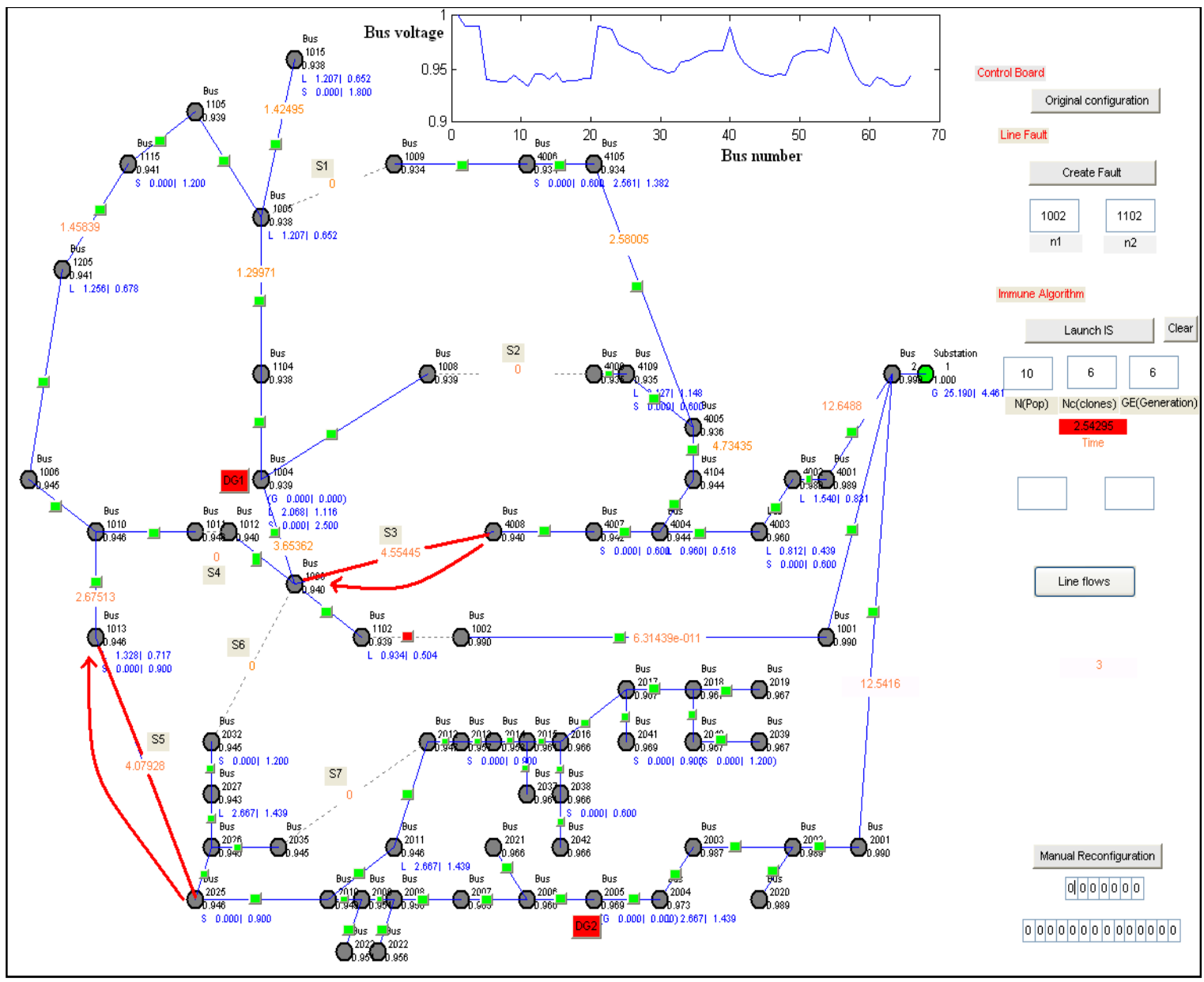

Figure 4.11 Reconfiguration using AIS algorithm

All these constraint helps the algorithm to compute the affinity and direct the algorithm towards the best reconfiguration solution.

In the biological immune system the destruction of the antigen once it faces the cell is done by part.

The cell does partial damages or destruction to the antigen till its final annihilation. The same thing is happening in the case of reconfiguration using the AIS, a partial healing is applied first to the system to restore some part of grid for example or at least to bring the system to a better state by reducing the congestion of the lines by performing low priority load shedding. As the fault occurs the AIS is triggered, and start rerouting the power to the out aged area. Figure 4.11 shows the path found by the AIS algorithm. We notice in this case that the algorithm has chosen to get the power from two different zones by switching on the switch number 5 and 3 . The figure shows also the voltage profile after performing the reconfiguration. 
By analyzing different combination of switching we found out that the choice or the strategy taken by the algorithm by dividing the power through two feeders is to relief the feeders since the amount of the power needed to supply the faulted area is about $8 \mathrm{Mw}$ which if brought form one feeder will result in huge losses and voltage drop under the limit. In this scenario the algorithm came up with a strategy in less than 3 seconds and by using the learning part of the algorithm the same strategy will be trigged instantly if the same fault occurs in the future. Figure 4.12, we show a manual reconfiguration of the system which simulates the human intervention in the real system. We can see from the figure that the operator has chosen to switch on Switch number 1 for example which is the simplest choice without any prior knowledge of the system. We can see that by choosing this combination the whole power is traveling through one area which causes a losses of about $1.68 \mathrm{Mw}$ and bad voltage profile with a minimum voltage of $0.866 \mathrm{pu}$. We realize that this action affects the whole grid and the entire system can collapse. In addition, even if the operator chooses to divide the power between the two feeders by using a combination other than the one applied by the AIS algorithm in this case switch number 6 and any other switch the voltage is still low Figure 4.13. 


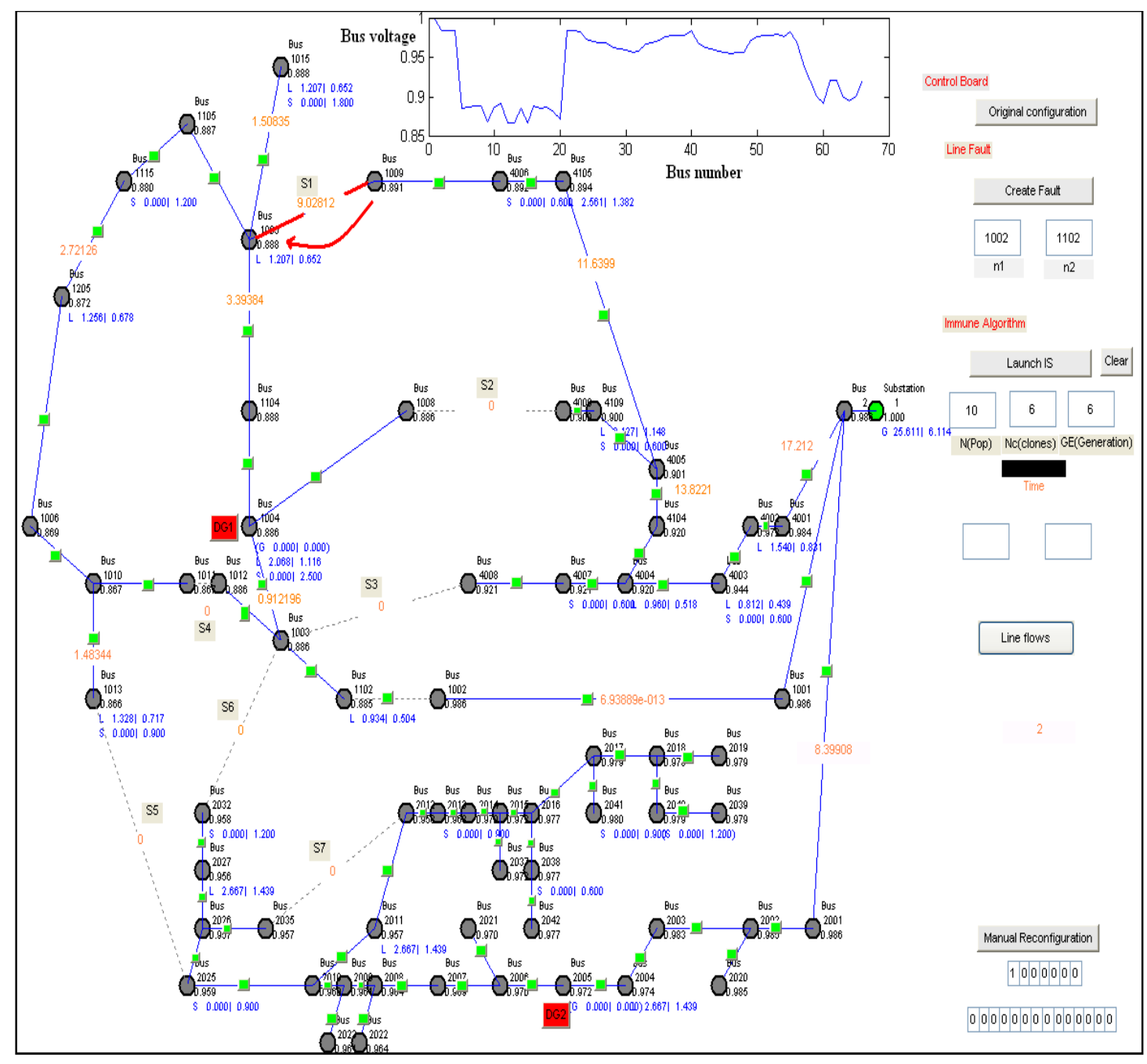

Figure 4.12 Manual reconfiguration (one switch operated) 


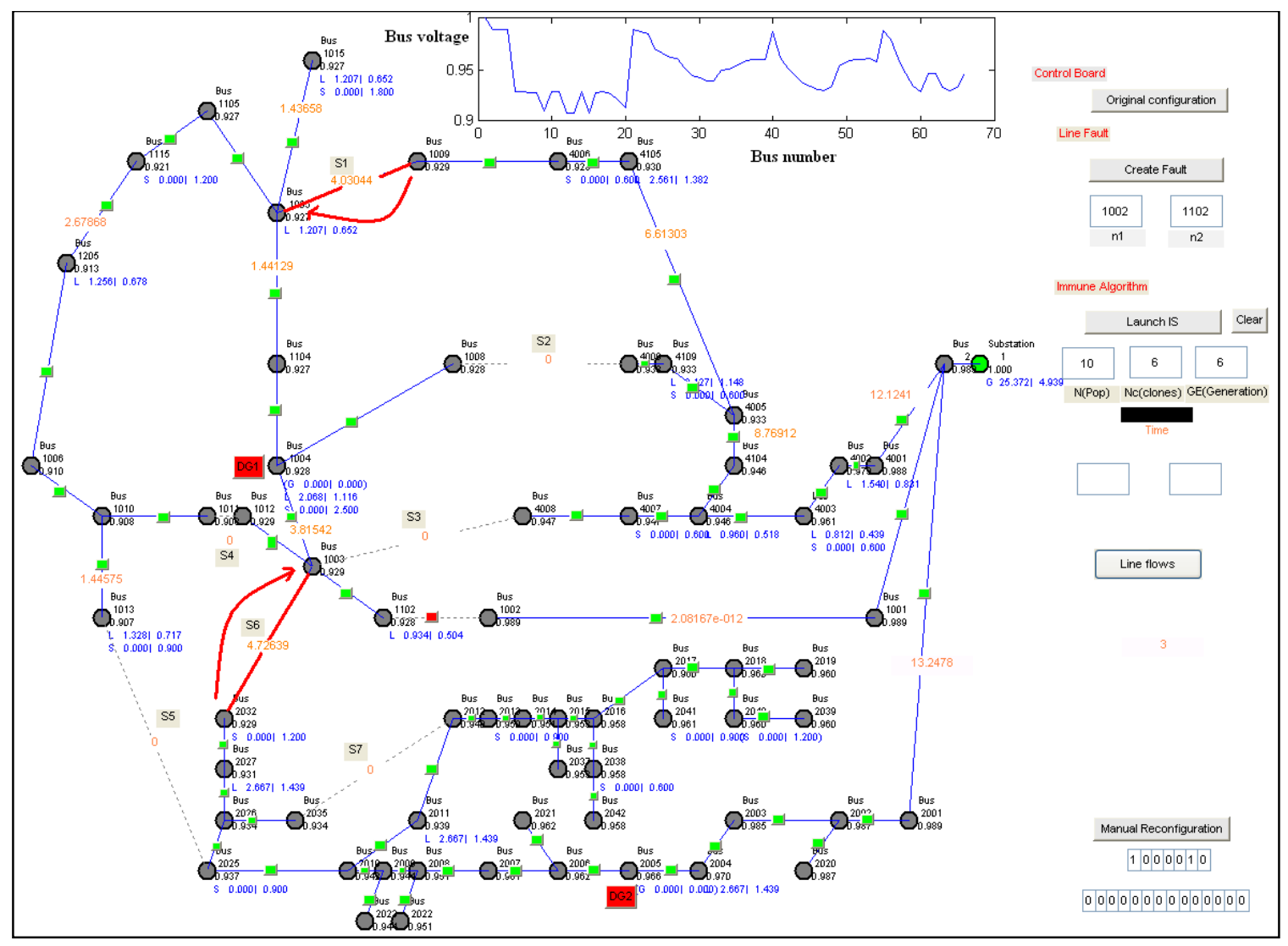

Figure 4.13 Manual reconfiguration (two switches operated)

\subsection{Hardware Prototype: A shipboard power system}

The hardware prototype that is used to demonstrate the MAS is a model of a power system of an all-electric naval shipboard, which consists of two AC generators, two AC propulsion loads, AC to DC converters, and DC distribution system [52]. The loads are divided into three zones. Zonal distribution reduces the amount of cabling and watertight penetrations required and simplifies damage control and maintenance. Each zone generally contains one or more load centers that supply power to electric loads in that zone [53]. The overall DC distribution system is divided into two DC buses namely port bus and starboard bus. These buses can be supplied from any of the main generator units through a Power Supply (PS). Breakers are used to isolate zones from each other. Each zone may contain an alternate power source and the load centers are capable of supplying the normal or alternate power to the loads. Each zone has two converter units, each connected to one bus. These units supply power to zonal loads. A schematic of a typical shipboard power system model is shown in Figure 4.14. 


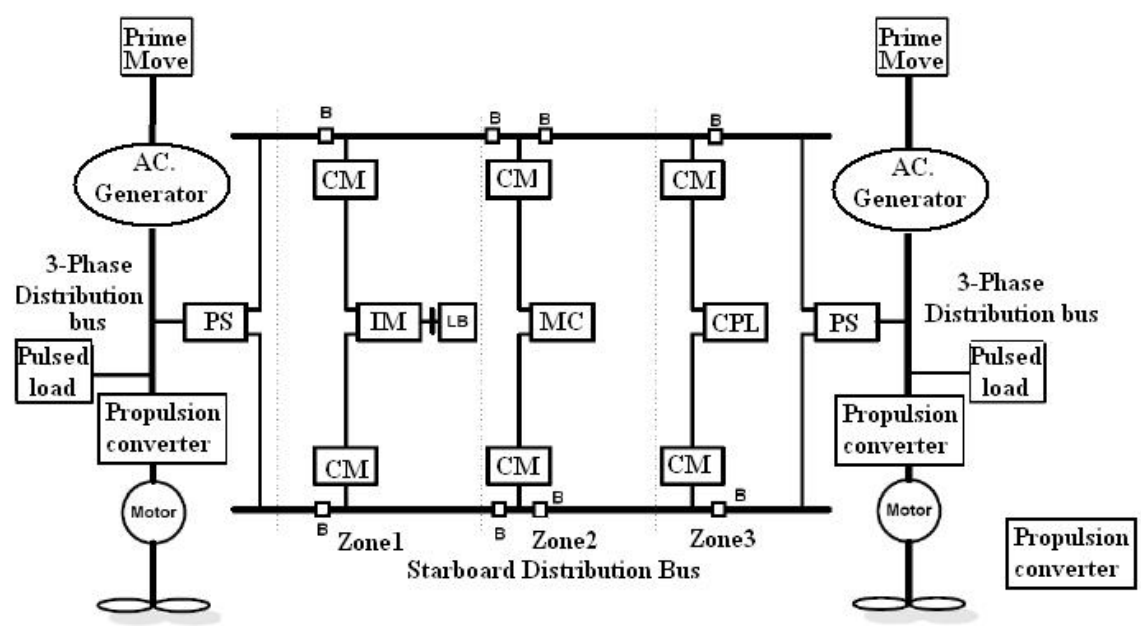

Figure 4.14 Shipboard power system

Where PS: Power Supply, CM: Converter Module, IM:Inverter Module, LB: Load Bank, MC: Machine Controller, CPL: Constant Power Load, B:Breaker. All the loads in the system are classified according to their priorities into three groups, vital, semivital, and nonvital. The Vitla loads are Nonsheddable loads that affect the survivability of the ship or life. Power to these loads is not intentionally interrupted as part of a load-shedding scheme. The Semivital Loads are important to the ship but can be shut down or switched to the alternate plant in order to prevent total loss of ship's electrical power. The Nonvital loads are Readily shedable loads that can be immediately disconnected without adversely affecting ship operations, survivability, or life.

In Figure 4.15 we show the deployment of the different agents throughout the shipboard power system 


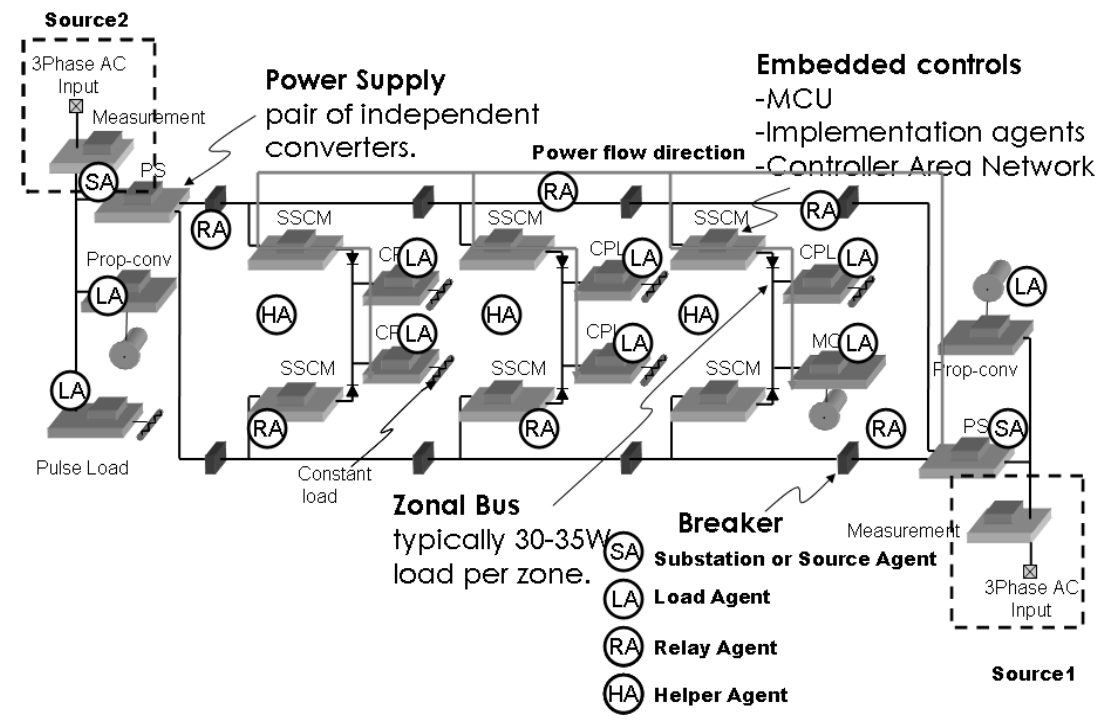

Figure 4.15 Agent deployment on the shipboard

The Simulation is carried out using Java Agent Platform Development JADE. JADE is a software framework fully implemented in the Java language. It simplifies the implementation of multiagent systems through a middle-ware that complies with the FIPA specifications. The platform allows designing and testing fully distributed multi-agent systems. The nonlinear shipboard power system model is implemented in MATLAB.

Two cases will be studied. The first case is to test the capability of the designed MAS in detecting and isolating a major fault on one of the feeders, and then perform a fast reconfiguration to restore power back to the loads. The second case is to test the capability in performing a load shedding after a major shortage in the power supply and restoring power to the loads according to their priorities.

Case 1: In this case the power system is in normal operation. All the three zones are supplied from the main generator. Assume that a fault occurs on the feeder between Breaker \#1 and Breaker\#2 as shown in Figure 4.16. As soon as the fault occurs, SwitchAgent\#1 senses the high current fault and start communicating with its neighbor SwitchAgent\#2 to locate the fault. In this case, SwitchAgent\#2 replies informing SwitchAgent\#1 that the fault is not seen in its range. Based on this information, both agents agree to open their breakers to isolate the fault. Note here that since the power can flow in both directions, both breakers have to open to allow restoration from the second generator. Furthermore, the agents have to know in which direction the power is flowing 
to decide on the way or direction of the communication. By opening the breakers, all three zones are blacked out as shown in Figure 4.17.

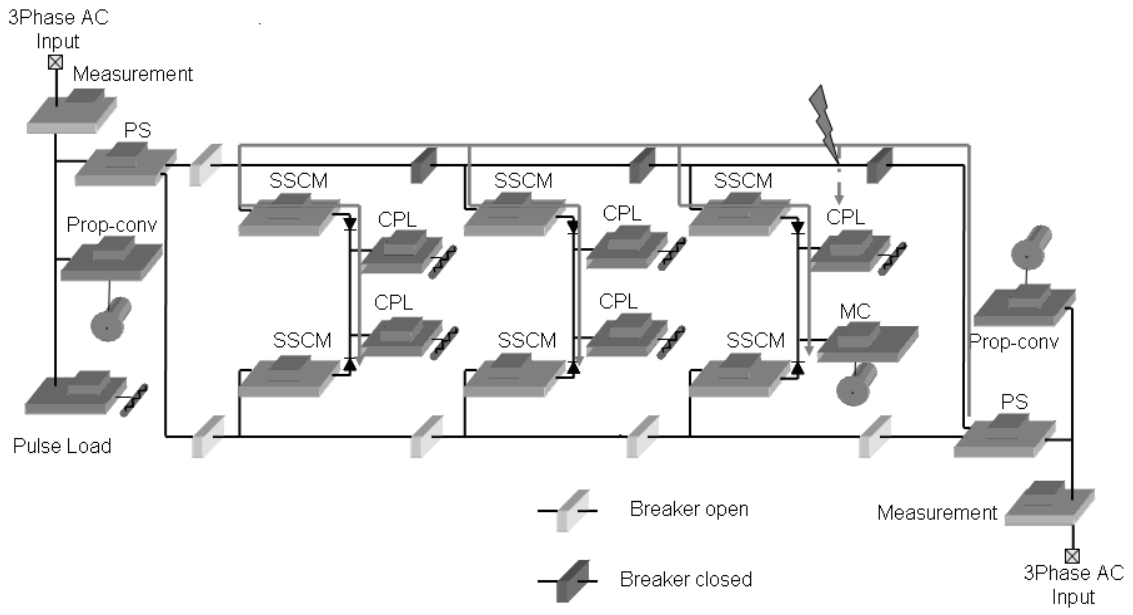

Figure 4.16 Fault occurrence at the feeder level

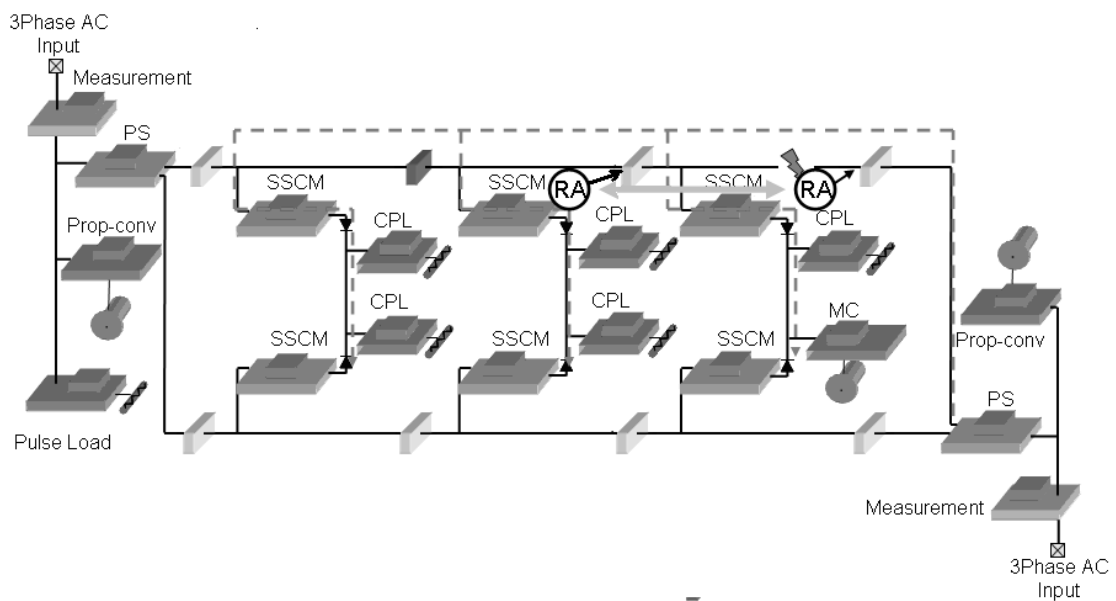

Figure 4.17 Fault detection and isolation by agents

As soon as the load agents detect the power loss, they start communicating with the neighboring SwitchAgents trying to restore the power. In this case SwitchAgents \#5, 6 and7 agree to energize the second feeder to restore the power to all three zones as shown in Figure 4.18. 


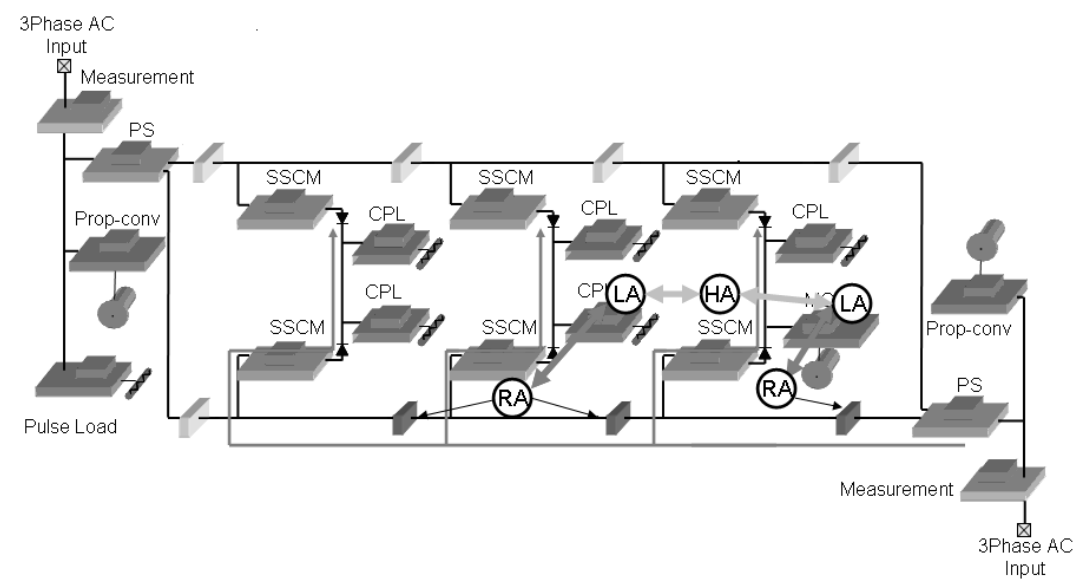

Figure 4.18 Reconfiguration and loads restoration

Figure 4.19 shows the current measured at both feeders and across load 3 of zone 2 during the process. We can easily recognize, from the currents profile, the fault isolation and reconfiguration restoration phases. A lot of information on the behavior of the system can be extracted by analyzing the measured currents. We can see from Figure 4.19 the fast response of the MAS. However, the results are based on a digital model which means the choice of the sampling time can affect the time measurements. Furthermore, the agents in the simulation are hosted in the Jade platform where no physical communication is considered to add the time delay of the signals exchanged. A more accurate measurement of the timing will be extracted later in this the work from the experimental results performed on the testbed. 


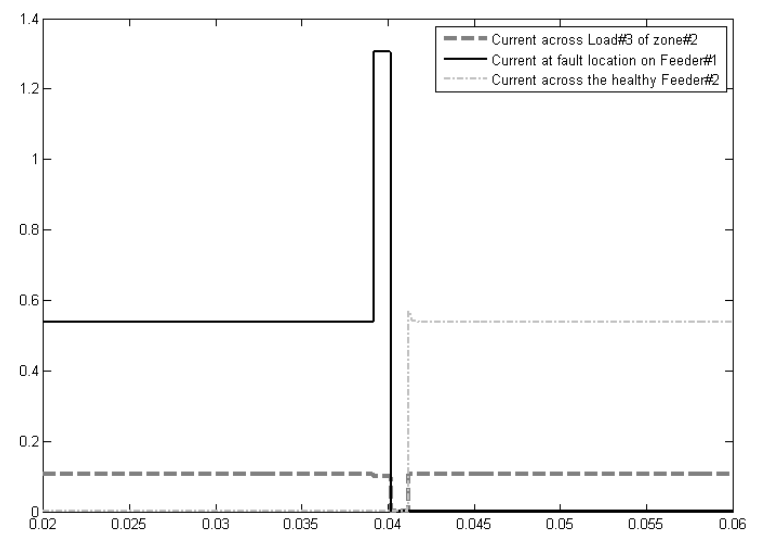

Figure 4.19 Current measurements at the fault location in Feeder\#1, at load\#3 and at the healthy feeder\#2

Figure 4.20 below shows some messages exchanged between the agents using the agent sniffing capability offered by the JADE platform.

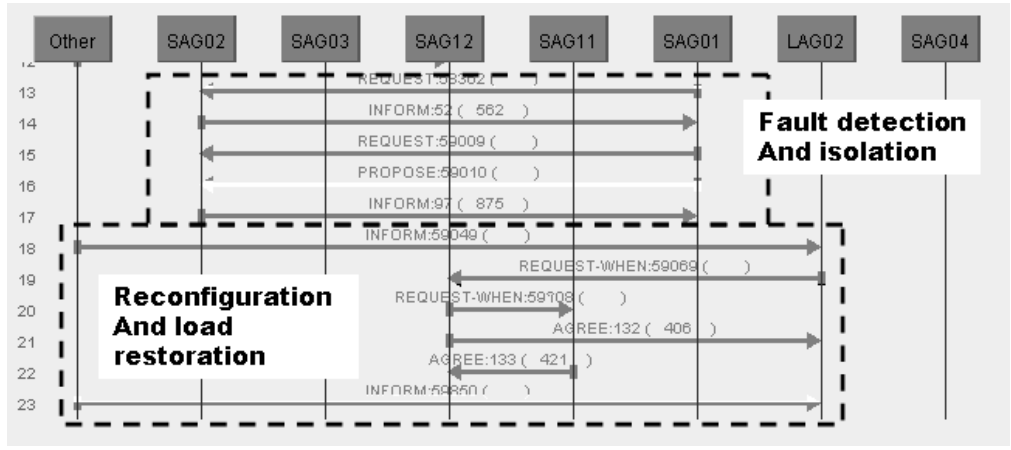

Figure 4.20 Messages exchanged between agents

Case2: In this case, the shipboard power system is operating in a normal condition when suddenly the generator can no longer supply all the three zones. In this case a load shedding process is initiated. The GeneratorAgent\#1 starts communicating with agent 2 to open breaker\#3 in order to shed zone3. Since the load is dynamically changing only agent\#2 knows the amount of power supplied to zone3, as shown in Figure 4.21. 


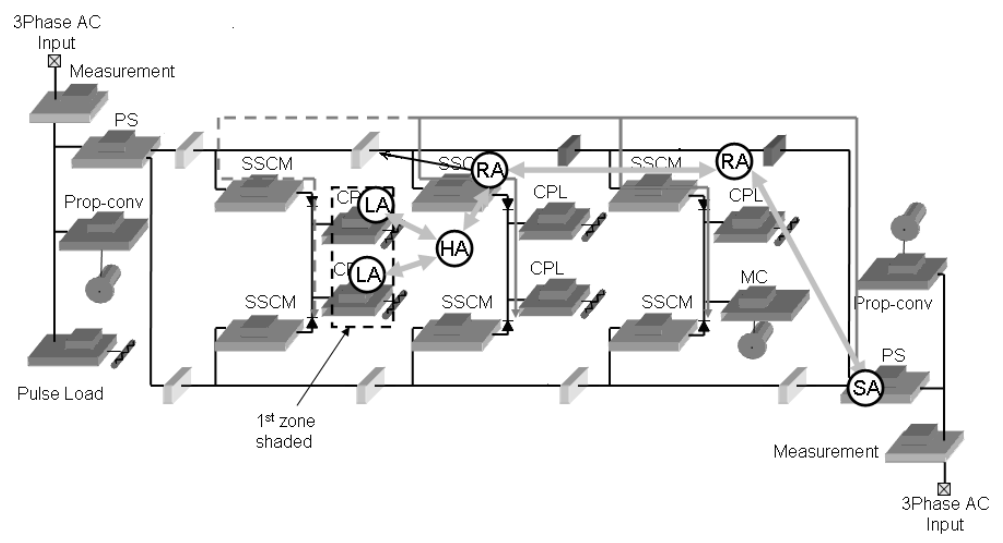

Figure 4.21 Shedding of Loads at zone\#3

After shedding zone3, the power demand still exceeds the capacity of the generator. GeneratorAgent1 starts communicating with SwitchAgent\#2 through SwitchAgent\#1 again in order to shed the next priority loads which are the loads in zone\#2. Agent2 sheds zone\#2 by opening breaker2. The remaining capacity is enough to supply the high priority loads in zone\#1 and the process of shedding is terminated as shown in Figure 4.22.

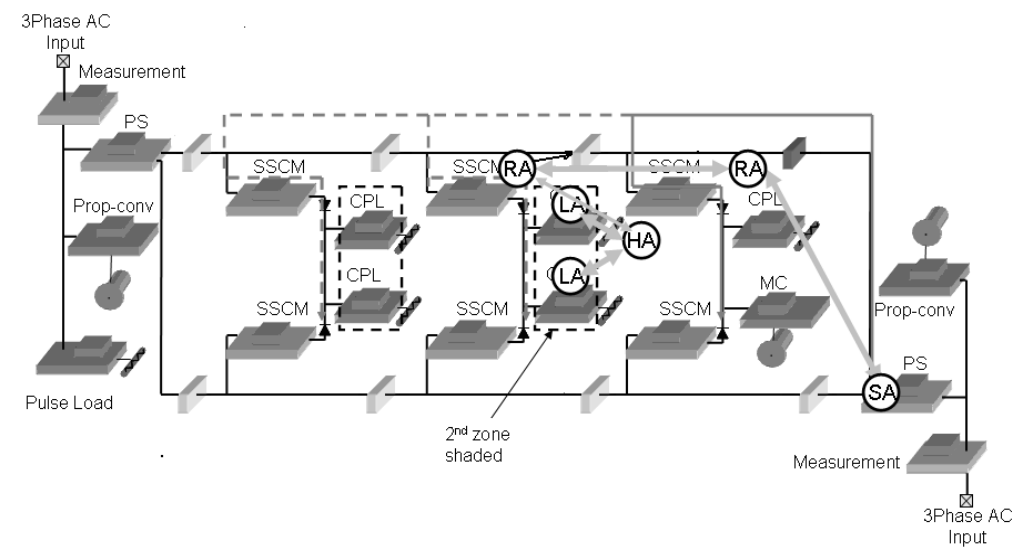

Figure 4.22 Shedding of Loads at zone\#2

As soon as the load shedding is terminated, loads in zone\#2 start communicating with neighboring SwitchAgents to get power from the second source. GeneratorAgent\#2 agrees, then breakers \# 3 and 4 are closed by their control agents respectively and power is restored to zone\#2. In this scenario generator\#2 has enough power to supply only zone\#2 since the pulse-load (weapon maneuvering) is connected directly to it. Therefore zone\#3 is left without power since it 
is a nonvital load as shown in Figure 4.23. Note here that if Zone\#2 was equipped with a distributed generator (DG) as a backup system, the agents would just get power from the DG and supply zone\#3 instead of using generator\#2.

Figure 4.24 shows the currents measured at load number\#2 of zone\#2 and the current flowing through breakers \# 1 and 2 of Feeder\#1 during the process of load shedding and restoration. Again we notice the fast response of the MAS system in solving the problem.

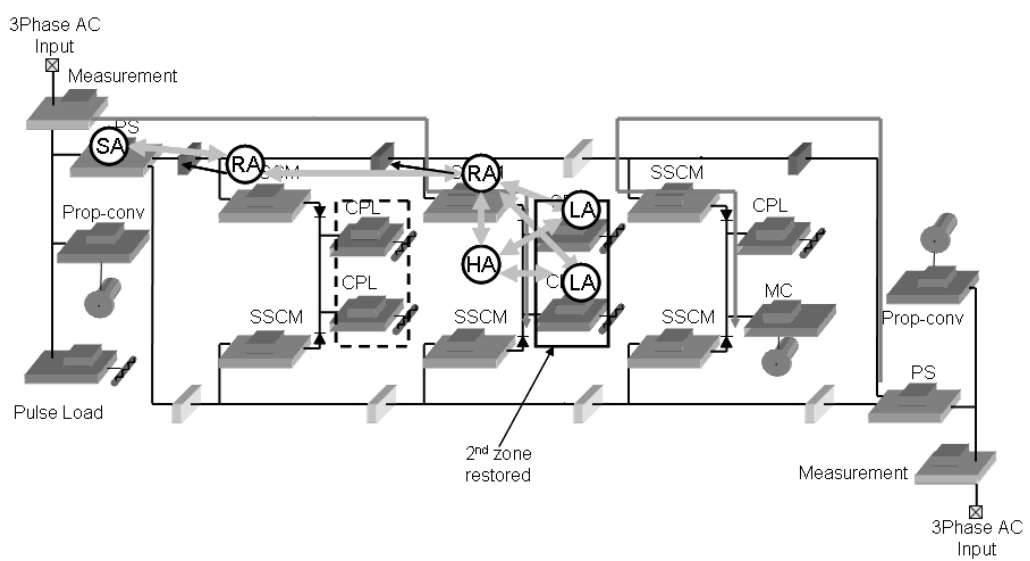

Figure 4.23 Power restoration to the loads of zone\#2

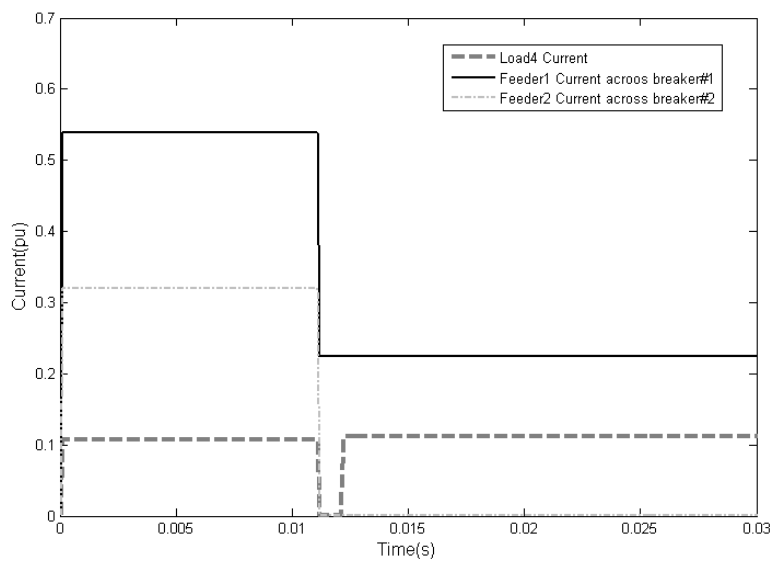

Figure 4.24 Current measurements 
The Figure 4.25 bellow shows some of the messages exchanged during the load shedding and load\#2 restorations.

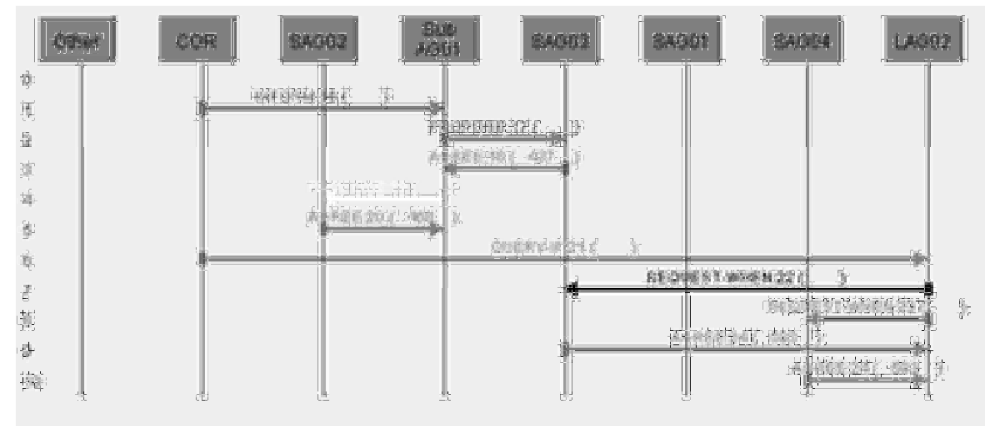

Figure 4.25 Message exchanged between the agents

\subsection{Experimental Implementation}

In this section the MAS is deployed on a real hardware testbed built by the Advanced Power and Electricity Research Center APERC group. The testbed provides a validation and demonstration platform for the local and distributed controls of the U.S. Navy's notional electric shipboard power systems. The testbed preserves the main characteristics of the Naval Combat Survivability (NCS) Testbeds, like the propulsion loads, pulsed loads, and zonal distribution architecture. Figure 4.26 shows a picture of the testbed hardware.

Each of the installed converters is equipped with its own embedded controller capable of providing analog-to-digital conversion, pulse-width modulation, interrupts, and timer support. Controls are implemented using a Freescale microcontroller MC9S12C32 running with an internal clock speed of 40MHz. 


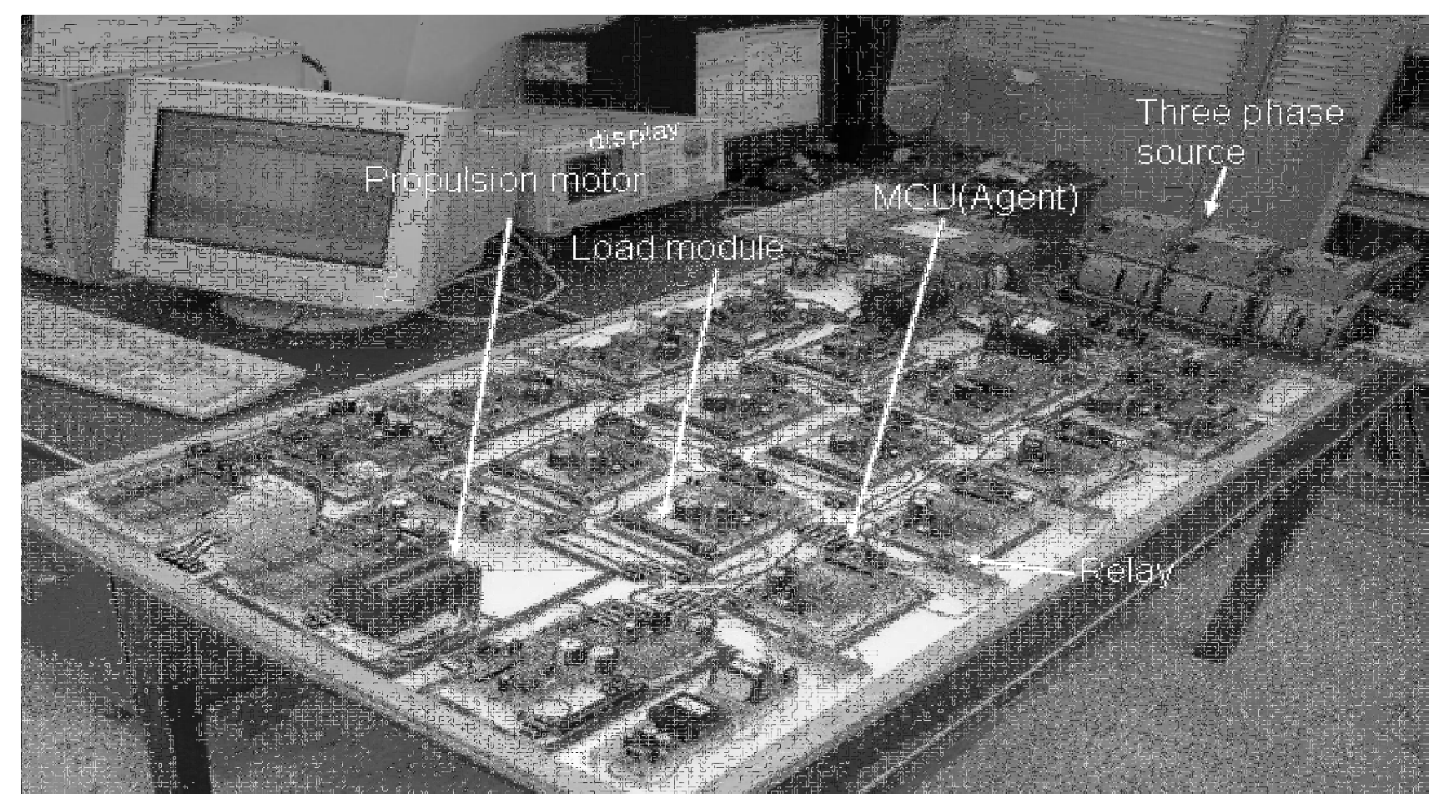

Figure 4.26 Assembled testbed hardware

The micro-controllers are used to implement the local embedded controls and provide hard realtime response capabilities. Each controller embodies or represents an agent. The development board used in this work is the CSM-12C32. The CSM-12C32 is a MC9S12C32 based development board manufactured by Axiom Manufacturing. This board offers on-board voltage regulator and a RS232 voltage level converter. It also offers two user programmable push-buttons and two user programmable LEDs. At least 31 input and output lines are made available through a 40 pin connector Figure 4.27. The figure also shows the entire propulsion module. 


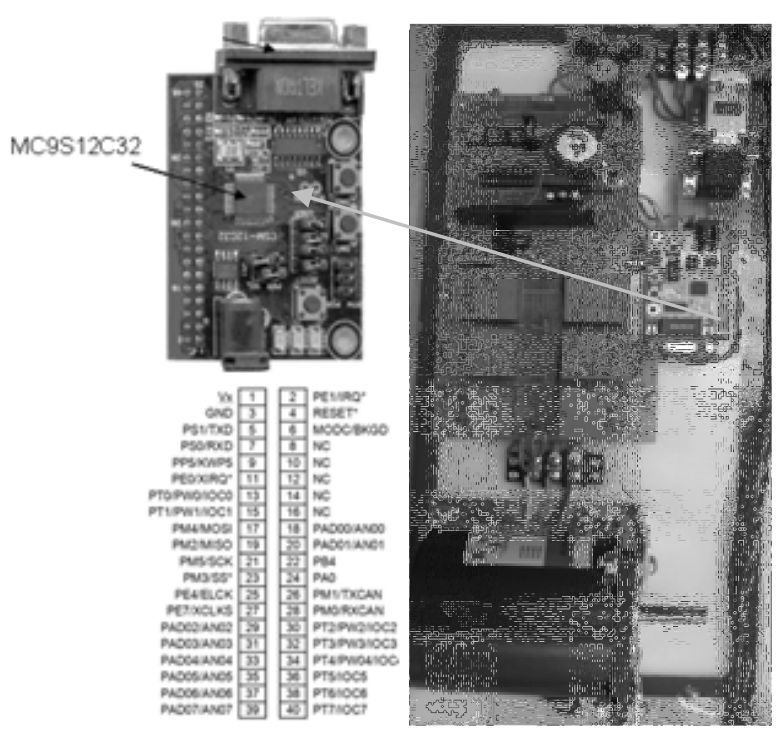

Figure 4.27 Assembled propulsion modules

The agent program is loaded into the microcontroller using CodeWarrior integrated development environment software for a fast design and prototyping.

The same cases studied and analyzed in the simulation are performed here.

In the first case we demonstrate the capability of the MAS detecting faults and performing reconfiguration. We assume that the power system was in normal operation. All the three zones were supplied from the main generator and suddenly a fault occurs on the feeder between Breaker $\# 1$ and Breaker\#2. Figure 4.28 shows the current measured across the feeder section where the fault happened and the current across the Load\#3 of zone2 using the YOKOGAMA PZ4000 Power Analyzer. 


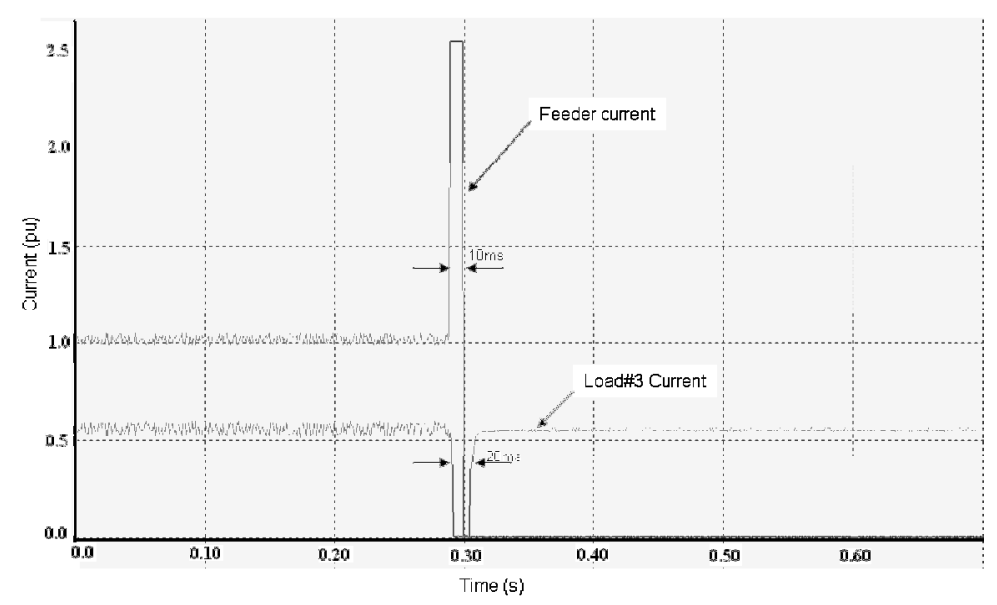

Figure 4.28 Measured current from the testbed

We can see from the figure the process of fault detection and isolation and the process of power restoration to the de-energized load. By analyzing the curves, we can extract the time it takes the agents to isolate the fault and restore power.

In this scenario the agents could detect and isolate the fault in about $10 \mathrm{~ms}$ and perform service restoration in less than $20 \mathrm{~ms}$.

The second case demonstrates the capability of the MAS in performing load shedding followed by restoration. Again assuming the shipboard power system in normal operation then a sudden fault happens at the generation level.

Figure 4.29 shows the currents at the Feeder\#1 and the current across load\#3 of zone\#2 during the process of load shedding and restoration. 


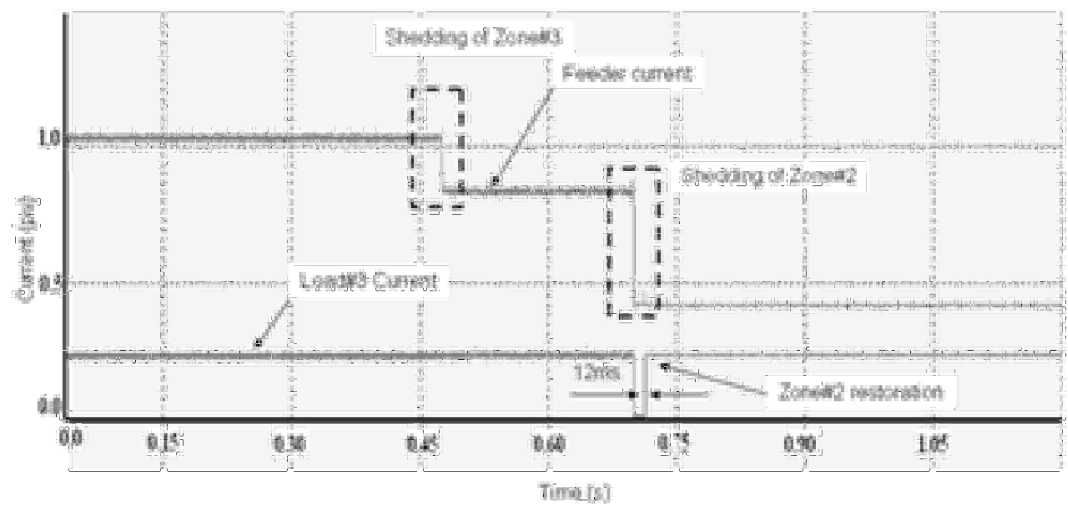

Figure 4.29 Measured current from the testbed

Again here the MAS system effectively and in a timely manner performed load shedding and restore power to zone\#2 through the second generator.

\subsection{Performance Evaluation}

To assess the performance of the Immune Based Multi Agent System, it is compared with a centralized optimization that uses global information about the entire system.

Figure 4.30 shows the voltage profiles of both approaches following a reconfiguration in case of a fault in Zone\#3 of the Southern California Edison Circuit of the Future test system.

It is noticed that the two profiles are very close. Also, the solution reached by the MAS is suboptimal since it's not performing an optimization on the entire system.

Figure 4.31 shows the profiles in case of severe outage in zone\#1 of the same circuit. Again, the voltage profiles using both approaches are very close and still above the voltage limit.

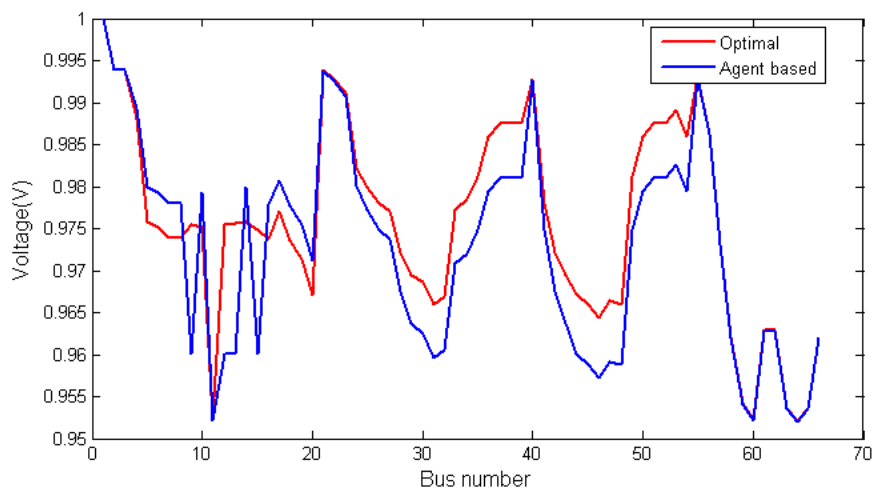

Figure 4.30 Voltage profile comparison (fault in Zone3 of the CoF) 


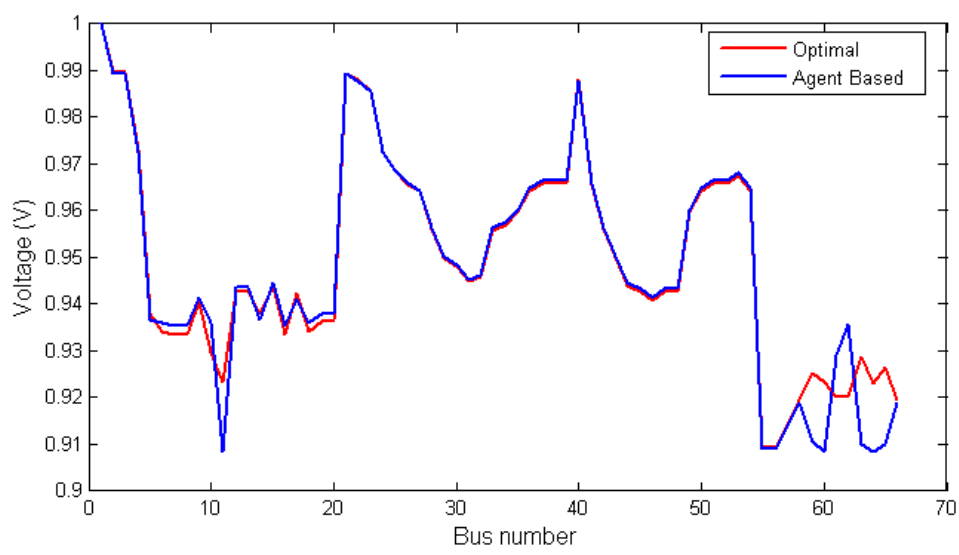

Figure 4.31 Voltage profile comparison (fault in Zone1 of CoF)

The simulation results obtained demonstrate that the proposed Multi-Agent System is able to find a suboptimal target configuration in all the scenarios considered. It is noted that the proposed approach could reach the right solution by making use of only local information and training received at the design level. The Multi-Agent System operates on the network in real time and takes about couple of milliseconds to find a target solution while the centralized approach might take minutes or even hours depending on the size of the system and cannot be applied in real time. Furthermore, the distributed nature of the Multi-Agent System makes it more reliable than the centralized one since the decision making is spread across the grid. Also, in case of a centralized approach the operators or control center can run into an overwhelming amount of data to process while in a distributed approach the data is locally processed.

\subsection{Conclusion}

In this chapter, we have designed and deployed a multi agent system on both terrestrial and shipboard power system for fault detection, isolation, reconfiguration, and load shedding purposes. In this work both simulation and hardware design and deployment of the MAS are addressed. The MAS design is based on the immune system algorithm in which the agents are trained and embodied with power system information. The MAS adapts to the change in the topology by communicating local information. The agents in this work are programmed in the Freescale MC9S12C32 microcontrollers and integrated in the testbed hardware. The simulation 
Chapter 4 Immune Based Multi-Agent System

and experimental results show that the agents are capable of performing very fast reconfiguration and load shedding schemes. 


\section{Chapter 5}

\section{SMART GRID SOFTWARE AND HARDWARE \\ DEVELOPMENT}

\subsection{Smart Grid}

The Electric distribution system is facing a great challenge by responding to the rapidly changing customer needs for electricity in the modern world. The increased use of electricity has increased the number of outages, voltage and frequency violations, and other power quality disturbances. In addition, rising interest in renewables, distributed generators and storage devices is forcing the power grid into modernization, or what we call the Smart Grid, to meet the demands of the 21 st century.

The Smart Grid will enable a high level of consumer participation to manage their electricity consumption similar to the banking system. Consumers will have a timely and clear picture on how much electricity they used down to the hour of the day instead of waiting for the monthly statements. The real-time pricing of the Smart Grid will enable the consumer to save money by using less power when electricity is more expensive. Furthermore, Smart grid encourages customers to own distributed renewable generation such as solar panels to sell the surplus of power to the grid and make profit. Also, smarter grid will help the environment by reducing the gas emissions through smart meters and renewable energies. Another benefit from modernizing 
the grid is the workforce generation. It is estimated that hundreds of thousands of jobs related to Smart Grid technologies will be created by government and utilities investment.

In today's grid a blackout can have a cascading effect that can affect banking, communications, traffic, and security. It is expected that a smarter grid will add resiliency to our electric power system and make it better prepared to address emergencies such as hurricanes, ice storms, and terrorist attacks.

Smarter grid technologies will detect and isolate power outages to contain them before they become rolling blackouts. They will also help ensure that electricity recovery resumes quickly and strategically-routing electricity to emergency services first, for example-after an emergency. In addition, the Smart Grid will take advantage of distributed generation to produce power when it is not available from utilities. By combining distributed resources, a community could keep its grocery store, police department, traffic lights, phone system, and health center operating during emergencies.

The electric power system, or grid, will also benefit from smarter grid technologies. The Smart Grid will result in:

More efficient transmission of electricity, quicker restoration of electricity after power disturbances, reduced operations and management costs for utilities through the integration of predictive analytics, self-diagnosis, and self-healing technologies, reduced peak demand, which will also help lower electricity rates, increased integration of distributed generation and renewable energy, and improved security.

The advances in microprocessors and communication technology can gear the power grid towards a modern and sustainable grid.

The US Department of Energy (DoE) has set a primary goal in modernizing the grid to support demonstration projects of key technologies that can serve as the foundation for an integrated modern power grid. They identify the inability to evaluate how the deployment will affect the electricity infrastructure as the largest barrier in the deployment of Smart Grid technologies [6].

In this work, we will develop and deploy smart grid technologies on laboratory Power Simulator hardware to address and analyze the effects of these new technologies before deployment in the real system as part of the West Virginia Super Circuit Demonstration Project. 


\subsection{West Virginia Super Circuit (WVSC)}

The West Virginia Super-Circuit (WVSC) project will demonstrate improved performance, reliability, and security of electric supply through the integration of distributed resources and advanced technologies. The goals of the WVSC project are noted below [54]:

- Seek to achieve greater than $15 \%$ peak power reduction in a cost competitive manner on the Allegheny Power (AP), West Run \#3 (WR-3) circuits located in the Morgantown, WV, service area

- Demonstrate the viability of advanced circuit control through multi-agent technologies

- Leverage advanced wireless communications to address interoperability issues between control and protection systems and distributed energy resources

- Demonstrate the benefits of the integrated operation of technologies such as rotary and inverter-based distributed generation, energy storage, advanced metering infrastructure (AMI), Price Driven Demand Response (DR), Automated Load Control (ALC), advanced wireless communications, and advanced system control technologies

- Demonstrate advanced operational strategies such as dynamic islanding and micro-grids to serve priority loads through advanced control strategies

- Demonstrate the reliability benefits of Dynamic Feeder Reconfiguration across several adjacent feeders

The principle component our team is developing is the Multi-Agent Grid Management System (MGMS). The MGMS is an agent based grid management system which will be composed of two main functionalities: Fault Location Isolation and Restoration (FLIR) and Microgrid Management System (MMS).

\subsubsection{Fault Location Isolation and Restoration}

In this phase, the team is expected to develop the hardware and software of the MAS to find fault locations at the zone level, isolate them, and restore service to un-faulted sections. Fault Location Isolation and Restoration system was deployed to WR-3 and WR-4 circuits through the previous DOE demonstration project (Morgantown DFT). Additionally, fourteen switches and two reclosers were installed on WR-3 and WR-4 feeders in the previous DFT project. 
The one line diagram of West Run feeders are illustrated in Figure 5.1 and Figure 5.2 below. The number and size of Zones on WR-5, WR-7, and WR-8 feeders have not been determined yet, so the diagram is representative.

In designing the algorithm, High Level Requirements are to be followed to ensure proper coordination with the rest of the system such as protection systems. Some of the requirements are [55]:

1 MGMS algorithms shall find, isolate feeder faults, and restore service to un-faulted Zones.

2 MGMS algorithms shall be triggered by a recloser lock-out event.

3 Service restoration to un-faulted Zones shall be completed in less than five minutes after the recloser locks out.

4 MGMS algorithms shall adapt to the new system topologies and perform its entire functions.

5 MGMS algorithms shall handle multiple non-simultaneous contingencies which are apart from five minutes.

6 MGMS restoration algorithms shall not violate any thermal or voltage limits.

7 MGMS restoration algorithm shall restore as much load as possible depending upon the source capacity.

8 MGMS shall find faults in radial and non-radial (system configuration with multiple energy resources) configurations.

9 MGMS FLIR algorithms shall take distribution generation, energy storage and controllable load into account when determining the restoration plan.

10 MGMS shall provide the interface between the ENMAC ${ }^{\text {TM }}$ system and the reclosers, automated switches and sensors.

11 MGMS shall be enabled/disabled by Allegheny Power operators remotely.

12 When MGMS is disabled, monitoring and manual control functions (by control center operators) shall still be allowed by the MGMS. 
Chapter 5: Smart grid software and hardware development

13 MGMS shall perform automatic switching operations to bring the system to the pre-fault configuration when a "Return to Pre-fault Configuration" signal is issued by the operator.

\subsubsection{Microgrid Management System}

In this phase, the team is expected to design a MAS to perform management of microgrids. The MAS will allow a microgrid -group of DER and controllable load to be able to disconnect from the main grid and operate without human intervention. The agents will be able to regulate the frequency and voltage of the microgrid in an islanded scenario. In addition to this, Microgrid Management System will be able to dispatch DER in order to accomplish a reliable and/or economical operation.

High level Requirements to be followed are [55]

1 MGMS shall optimally dispatch distributed generation, energy storage, and controllable load to reduce the annual projected peak load by $15 \%$. Projected load will be provided by Allegheny Power.

2 MGMS shall optimally dispatch distributed generation, energy storage, and controllable load when operators issue a command to initiate load reduction in the system. MGMS shall be able to accept the signal coming from the control center Human Machine Interface (HMI).

3 MGMS shall dispatch DER with different cost functions optimally when excess capacity exists.

4 MGMS shall make the islanding decision from the main grid.

5 MGMS shall allow the islanded microgrid to reconnect to the main grid without taking an outage.

6 MGMS shall dispatch DER to meet active and reactive power demand at all times in an islanded scenario.

7 MGMS shall regulate the frequency of the islanded microgrid within [TBD] band.

8 MGMS shall regulate the voltage of the islanded microgrid within 112-127 V (phase-toneutral) band. 
9 MGMS shall be able to expand the borders of the microgrid when there is excess generating capacity present within the microgrid.

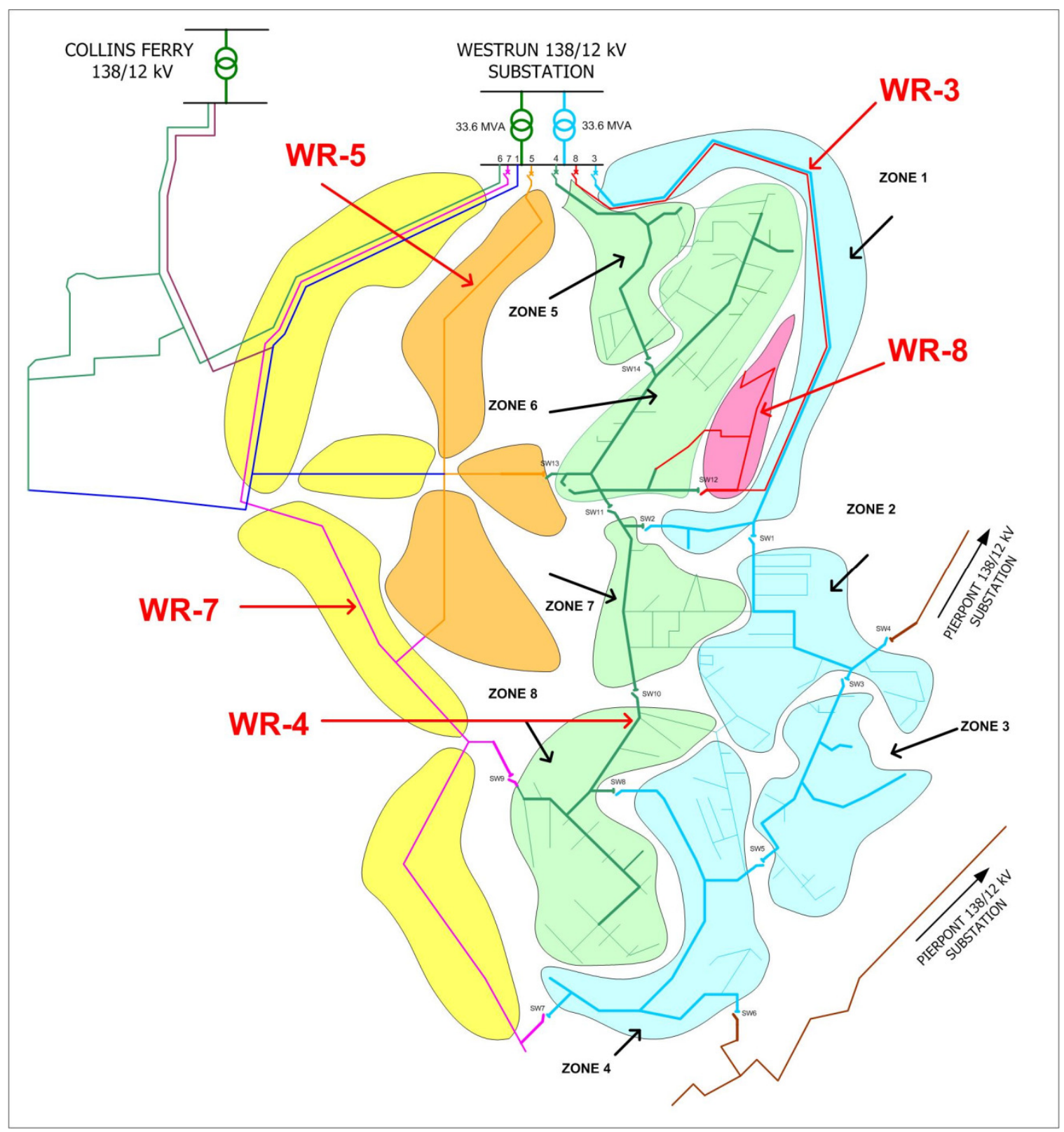

Figure 5.1 One line diagram of the WVSC 


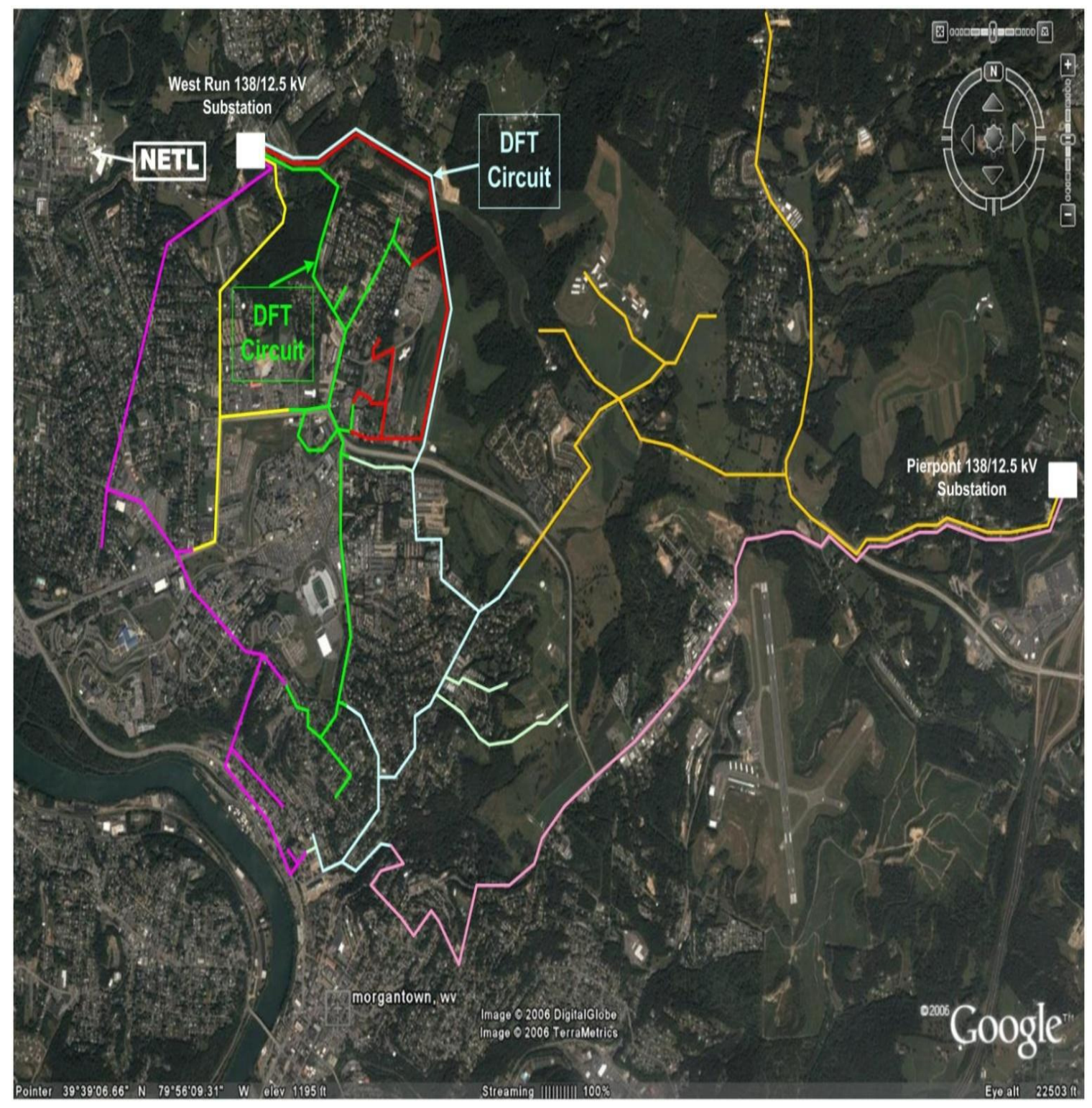

Figure 5.2 Google earth picture showing the WVSC layout 


\subsection{Software Implementation On the WVSC}

In the following, the Multi Agent System designed is implemented and deployed first on the Java Agent Development Platform (Jade) a long with a nonlinear dynamic three phase model of the two feeder W\#3 and W\#4 of the West Virginia Super Circuit for testing and debugging the algorithm before the hardware implementation on the Power Simulator. The agents in this case sense and act on the system in real time. Since the WVSC is already equipped with electronic switches that allow fault management and reconfiguration, an agent zonal architecture is adopted. A feeder section is called a zone if it can be interconnected through one or more electronic switches. A zonal architecture would have a Zone Agent in each zone of the feeder and a Switch Agent at each electronic switch. An example of a zonal architecture is illustrated on one of the WVSC feeder lines, shown in Figure 5.3 Agent Zonal Architecture.

The zoning of the feeder allows a cost effective automation of the circuit.

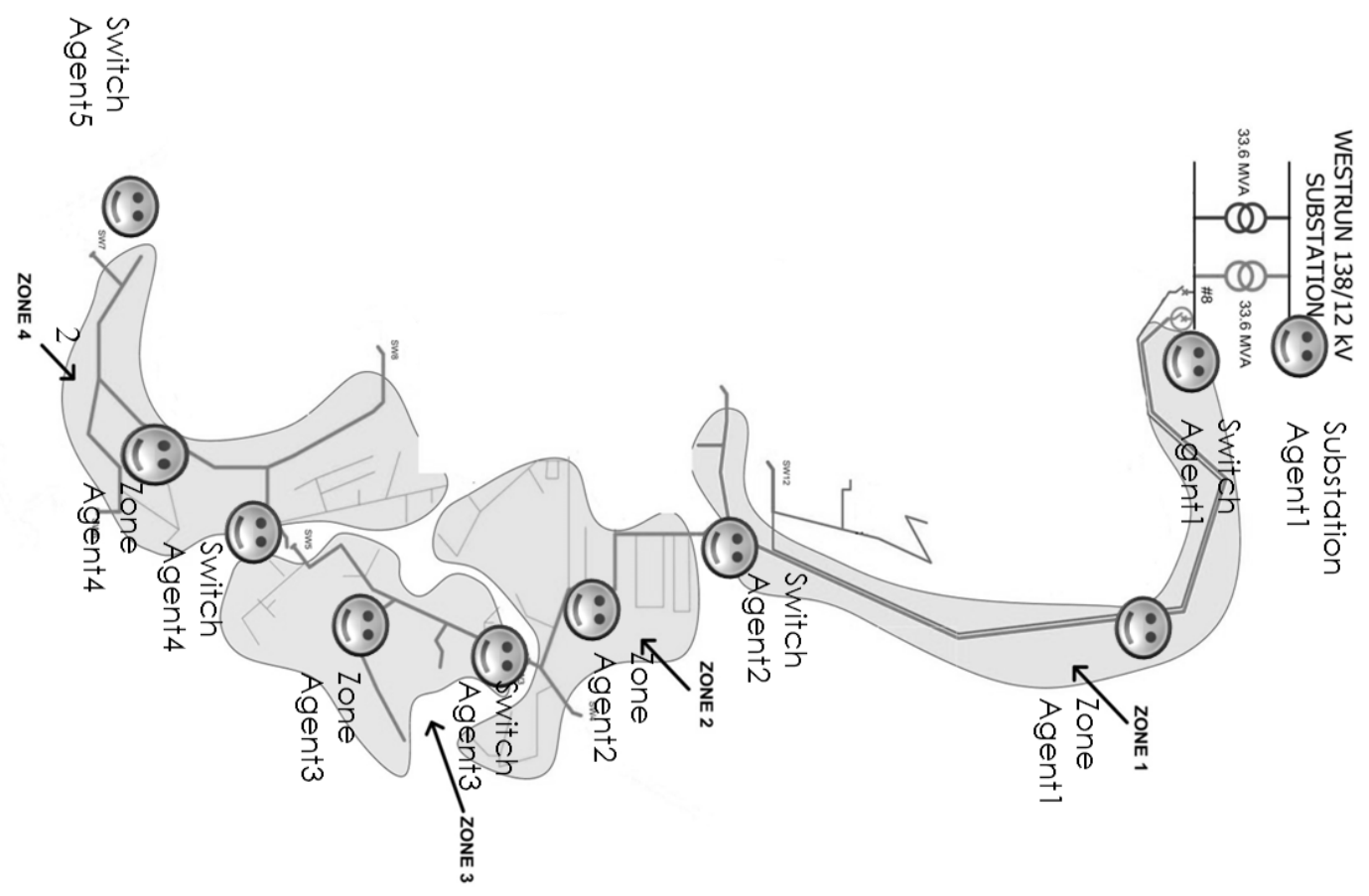

Figure 5.3 Agent Zonal Architecture

In this scenario, a three phase permanent fault is applied in zone\#6 of West Run feeder W\#4 (see Figure 5.1). The figures below show the effect of the fault on the system and the action taken by the Multi Agent System trying to detect, isolate the fault, and restore power to the healthy zones. 
Figure 5.4 depicts the waveform of the currents and voltages seen at the substation. We can observe from the figures the response of the protection system which is in this case an electronic recloser situated at the substation level. Prior to the fault the feeder was energized from both the substation and the rotatory DG situated in zone\#8. At the moment the fault occurs, a signal is sent from the agent residing by the recloser to the DG Agent in order to trip the DG to avoid any damage (following the requirement for DGs integration to the grid) as shown in Figure 5.7 Current and Voltage in zone\#8. After the lockout signal from the recloser is received, the agents on the feeder perform fault detection using the recoded current and voltage data during and prior to the fault. Then, the faulted zone is isolated as illustrated in Figure 5.5.
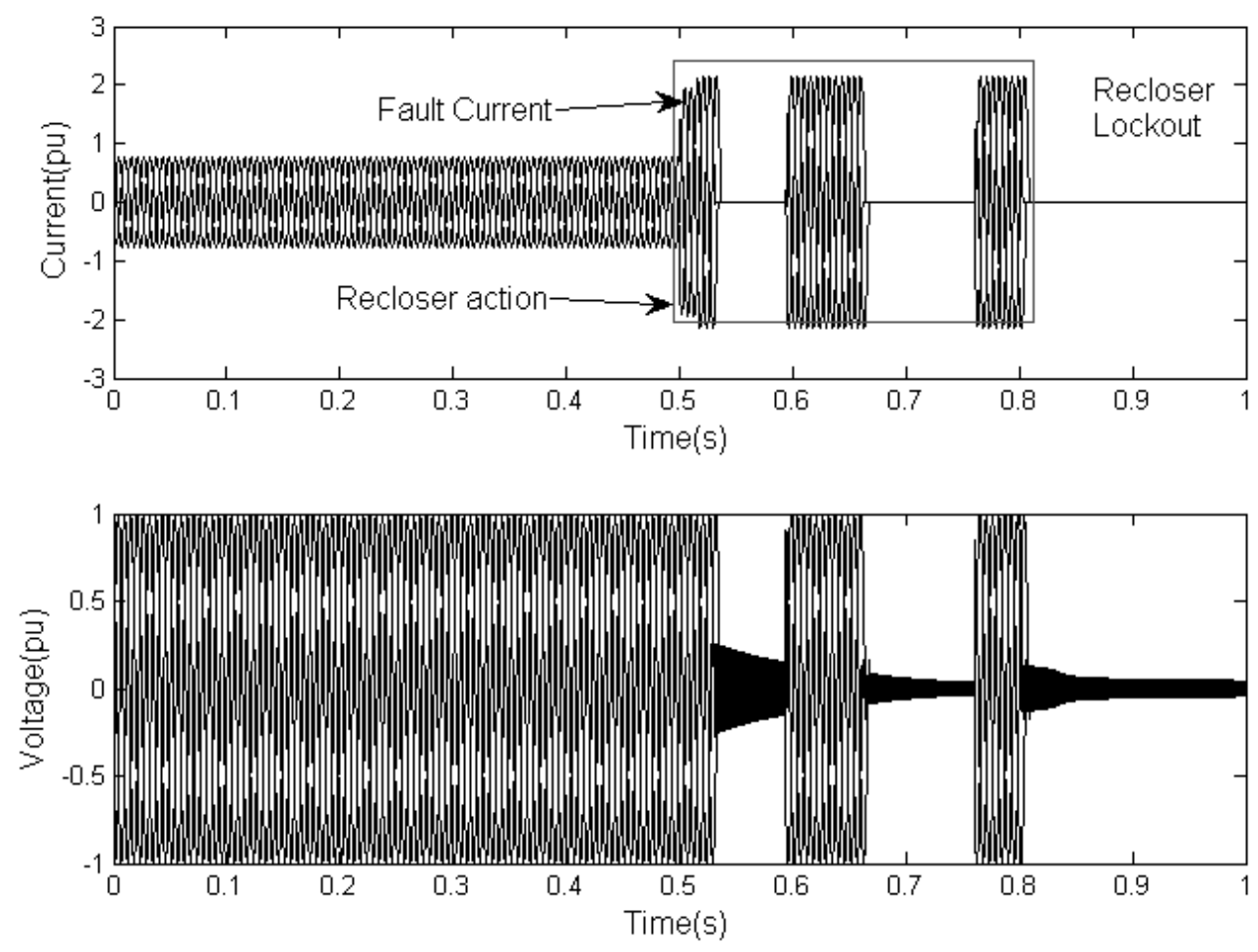

Figure 5.4 Current and Voltage at the substation 

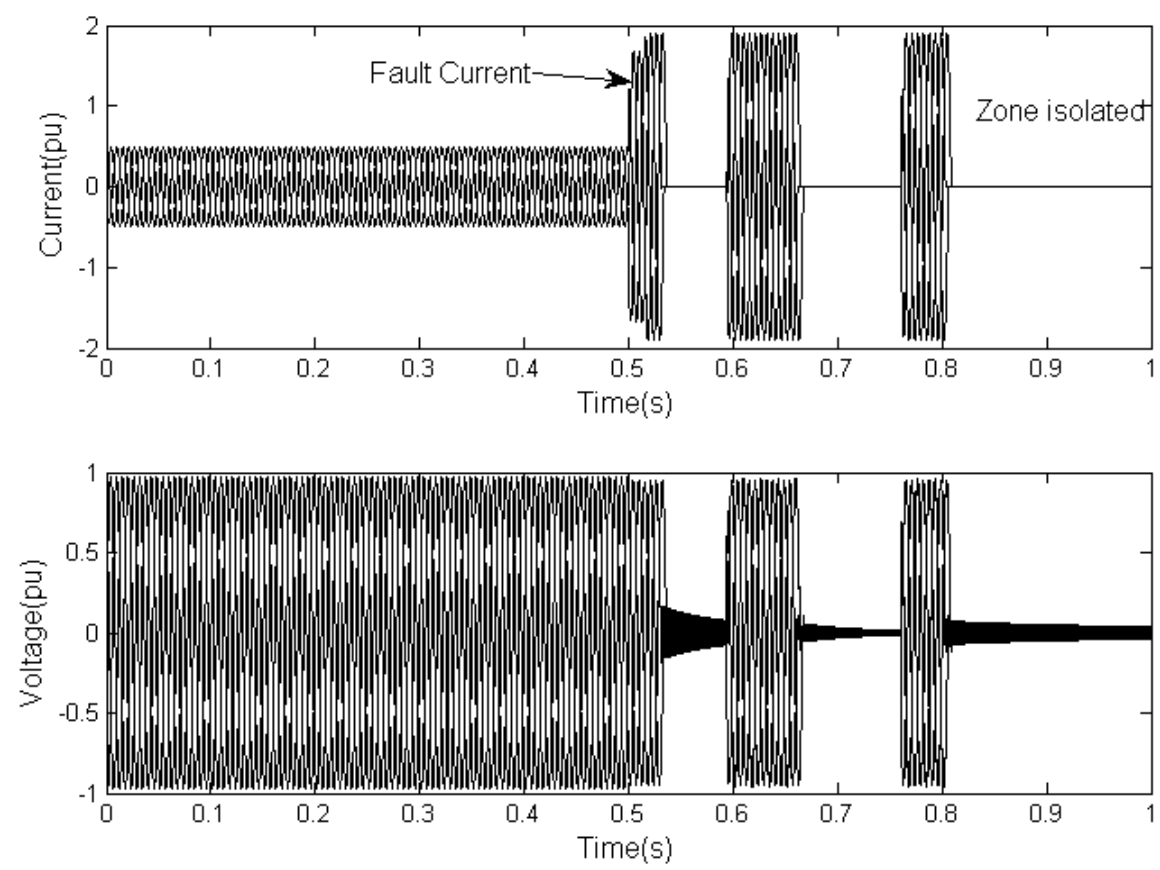

Figure 5.5 Current and Voltage in the faulted zone\#6

Once the faulted region is isolated, the information is then transmitted by the Zone Agent in Zone\#6 to the neighboring zone agents to start the process of reconfiguration to restore the rest of the system. As soon as the neighboring agents receive the fault information, they start reconfiguring the circuit based on the training and measurements in a downstream fashion starting from the zones that are closer to the substation. In our scenario, the Zone Agent in Zone\#7 starts the restoration by requesting power from the neighboring feeder W\#3, as shown in Figure 5.6. Once this operation is terminated the information is sent to the Zone Agent in Zone\#8. Zone Agent in this Zone\#8 chooses to isolate its zone and requests the DG to reconnect, as depicted in Figure 5.7. 
Chapter 5: Smart grid software and hardware development
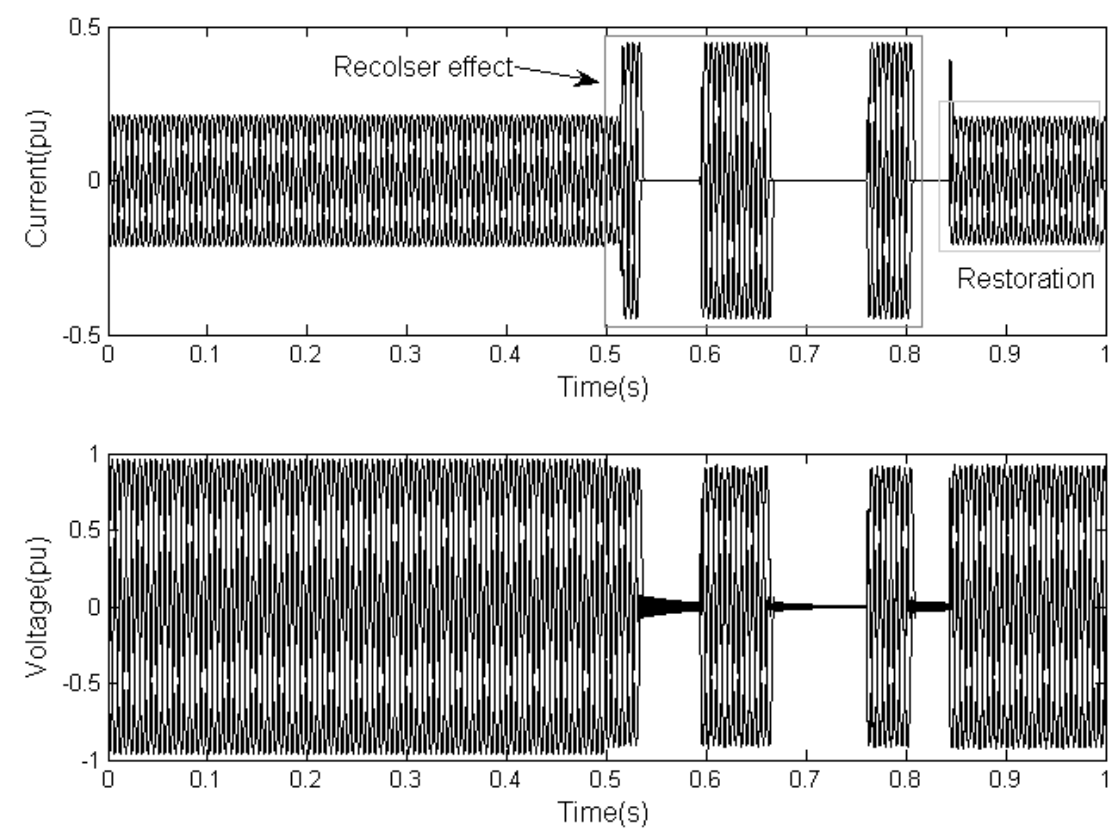

Figure 5.6 Current and Voltage in zone\#7
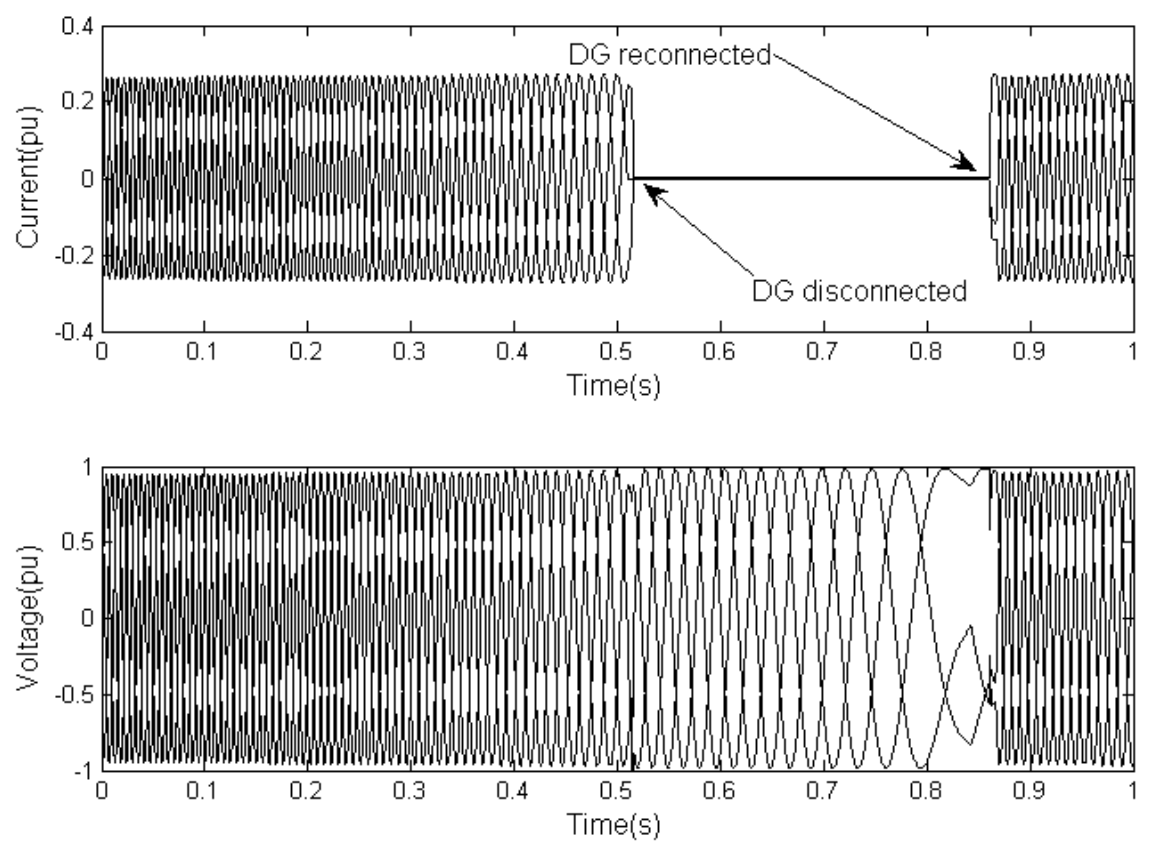

Figure 5.7 Current and Voltage in zone\#8

The inter Agent communication during the entire scenario is shown in Figure 5.9.

In the following, the same scenario is performed without prior training of the system to compare the Multi Agent System proposed in the literature by [2-6].

Since no overloading of the system is expected, the agents in this case, restore both zones\#7\&8 from the neighboring feeder $\mathrm{W} \# 3$. Because no training for voltage violations was 
offered to the Agent at the design level this strategy resulted in voltage violation or voltage drop below the limit in zone\#8 as shown in Figure 5.8.

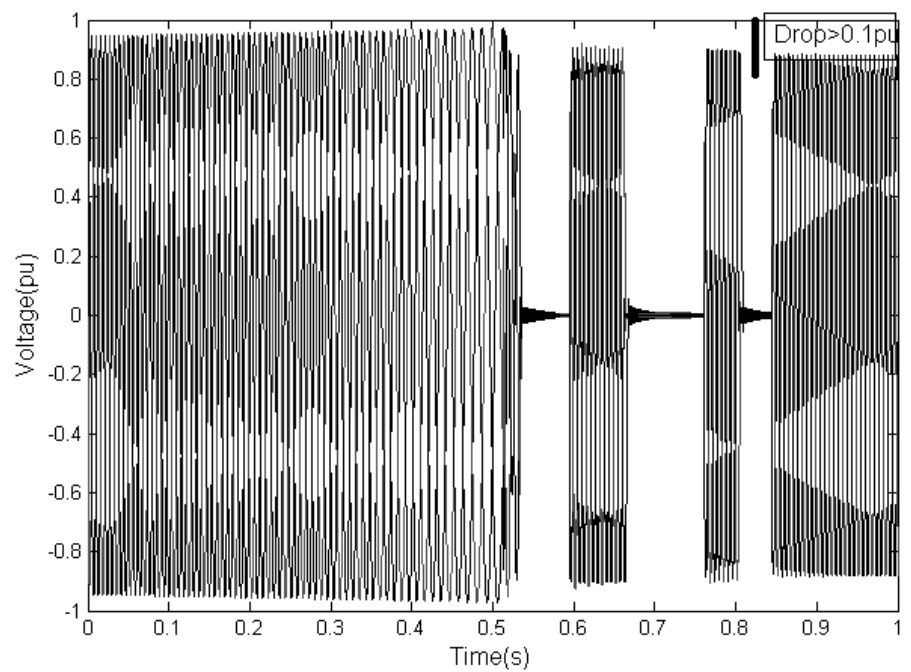

Figure 5.8 Voltage in Zone\#8

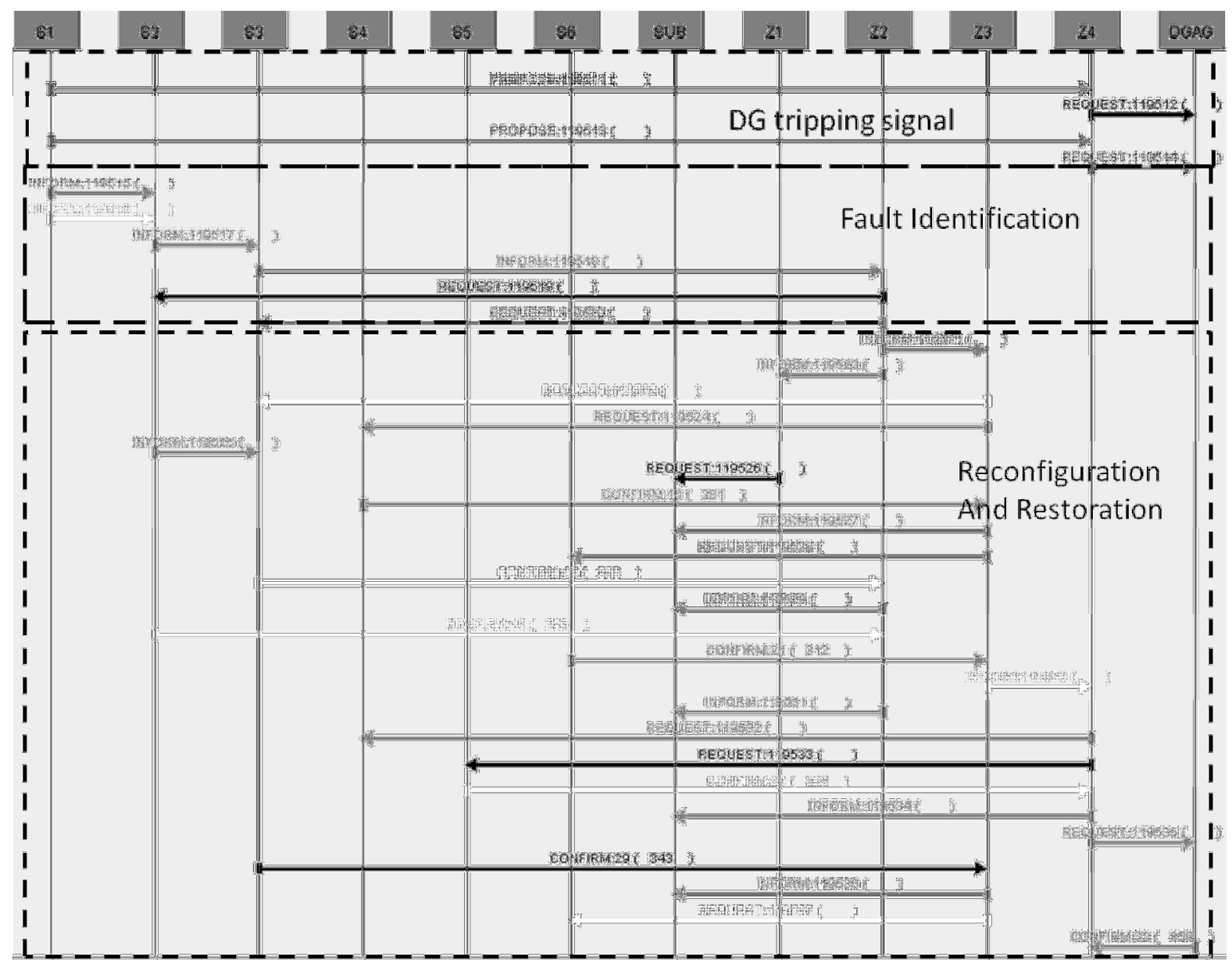

Figure 5.9 Inter Agent Communication 


\subsection{Power Simulator Hardware}

The Lane Department of Computer Science and Electrical Engineering at West Virginia University possesses an analog power simulator installed in the 1970s. The simulator, as shown in illustration Figure 5.10 and Figure 5.11, is a low power hardware replica of a distribution system that contains commercial, industrial and residential loads. Power can be supplied to the loads from different internal and external circuits and generators and routed in a variety of ways. The current objective is to design or transform the Analogue Power Simulator with its old technology to a smart power grid simulator by loading it with digital relays, controllable electronic switches, distributed energy resources, smart microprocessor based agents, and a two way wireless communication platform.

The new digital hardware will be integrated with the older electromechanical hardware as is found in real world power systems which once completed would give us a great laboratory tool for testing and developing smart grid technologies.

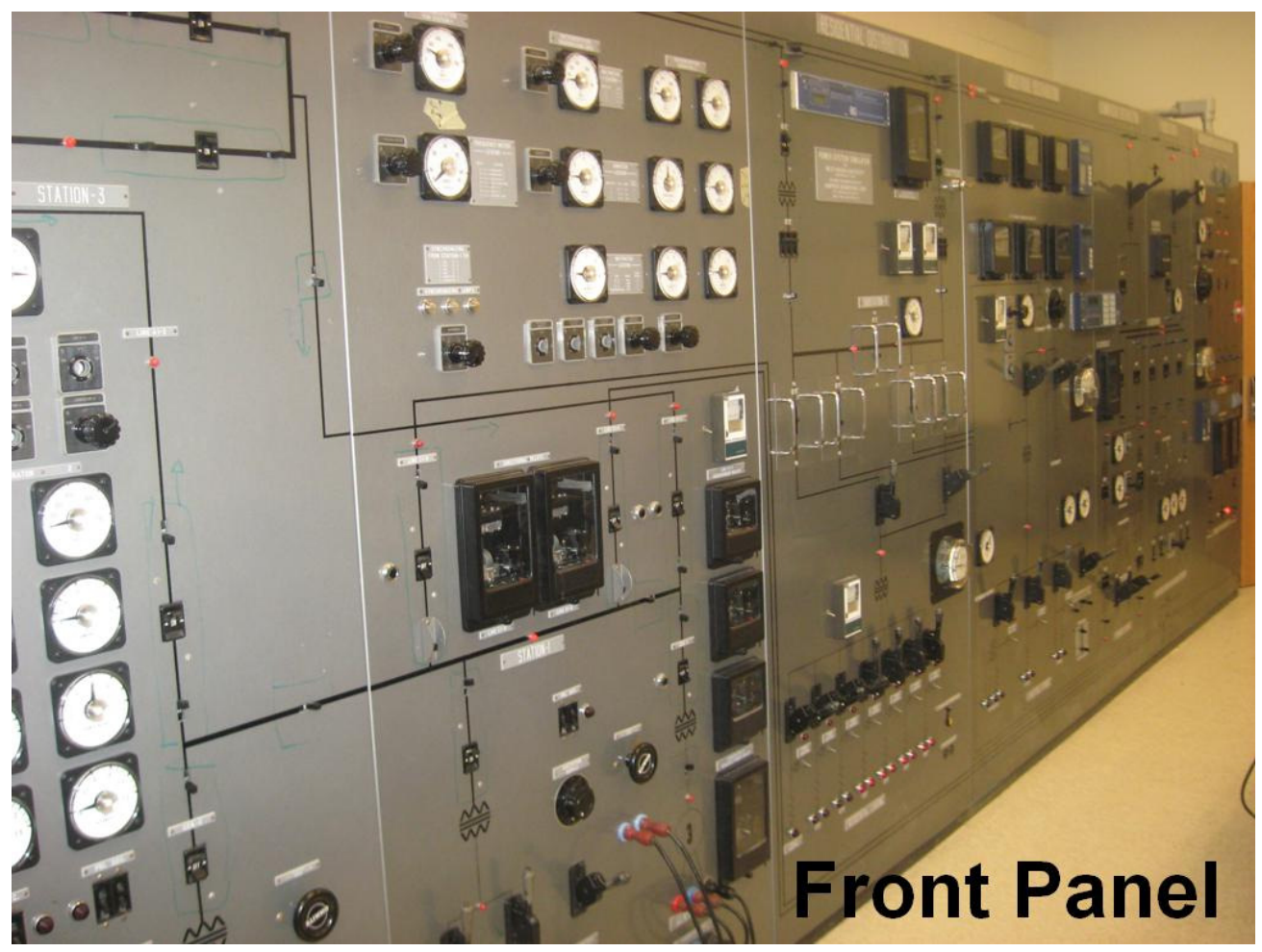

Figure 5.10 Power Simulator Front Panel 


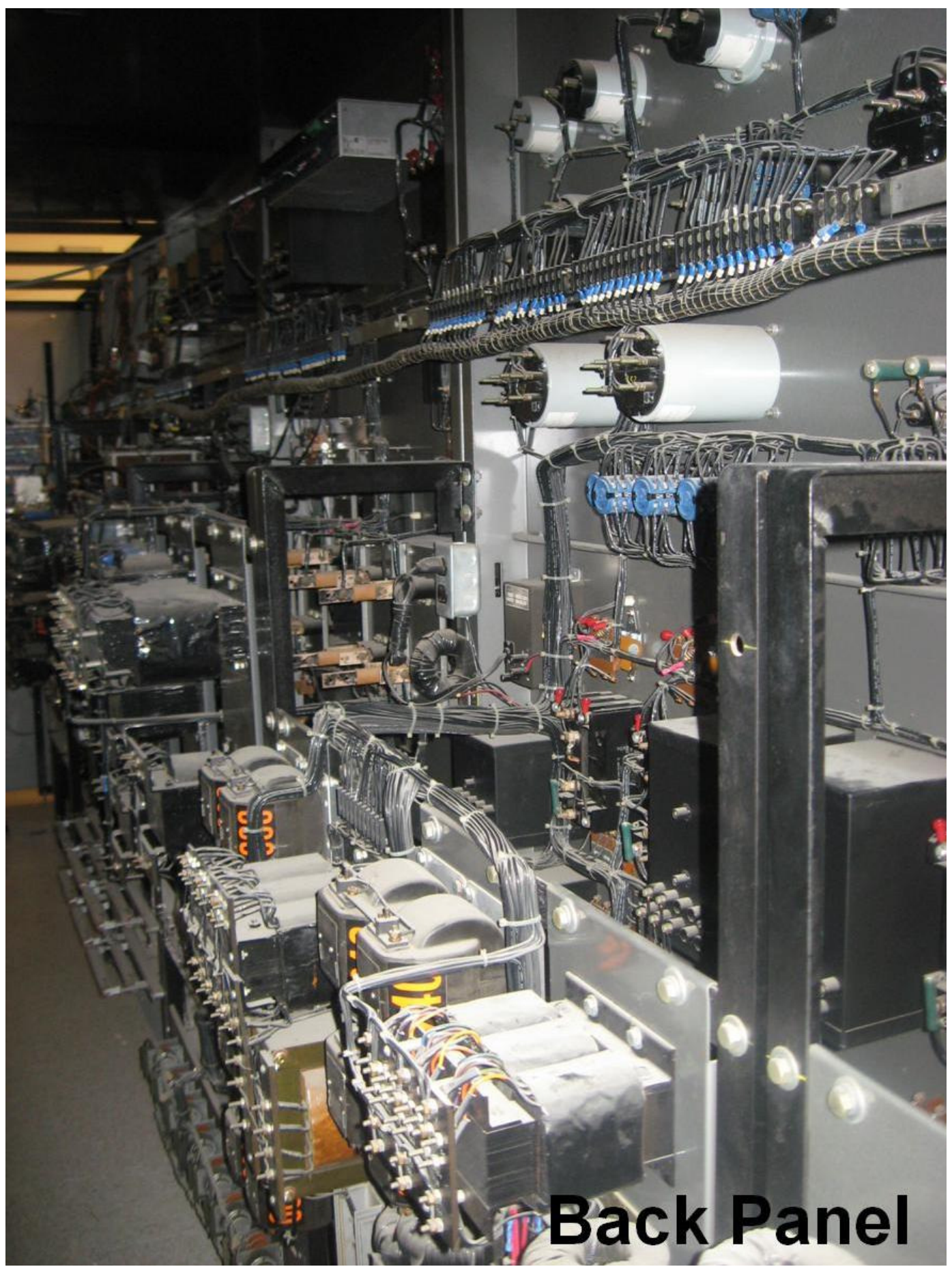

Figure 5.11 Power simulator back panel

In Figure 5.12, we are showing the one line diagram of the simulator circuit with the expected distributed components to be installed. At this point a small scale Multi-Agent system network is installed on the circuit along with an electronic recloser and a wireless communication network 


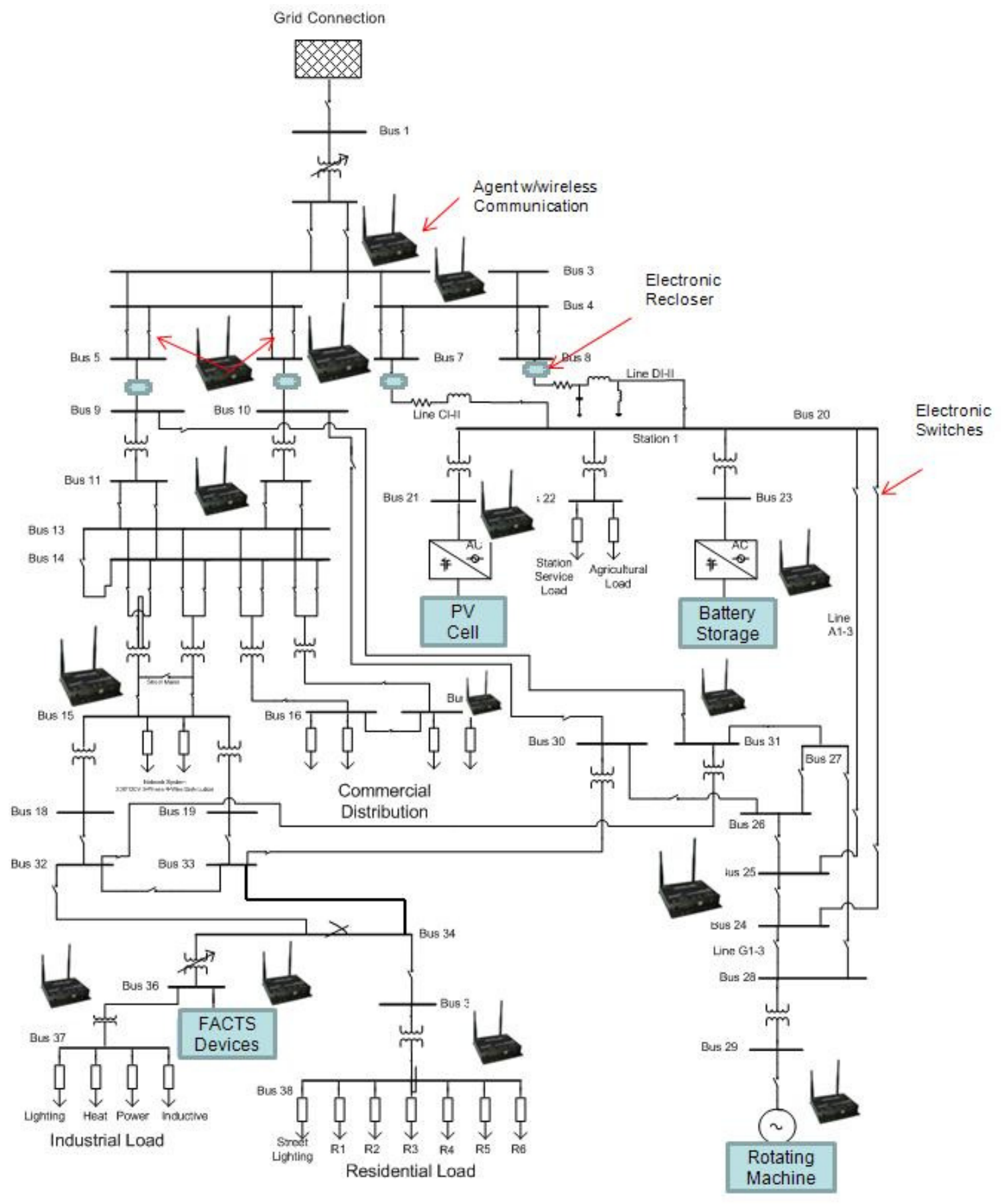

Figure 5.12 One line diagram of Power Simulator 
5.5 Hardware Agent Design and integration

5.5.1Microprocessor hardware

MCU Development Module (CSM-12C32):

The CSM-12C32 is an evaluation or demonstration module for the Freescale (Motorola) MC9S12C32 microcontroller. We will be using this module in C language programming.

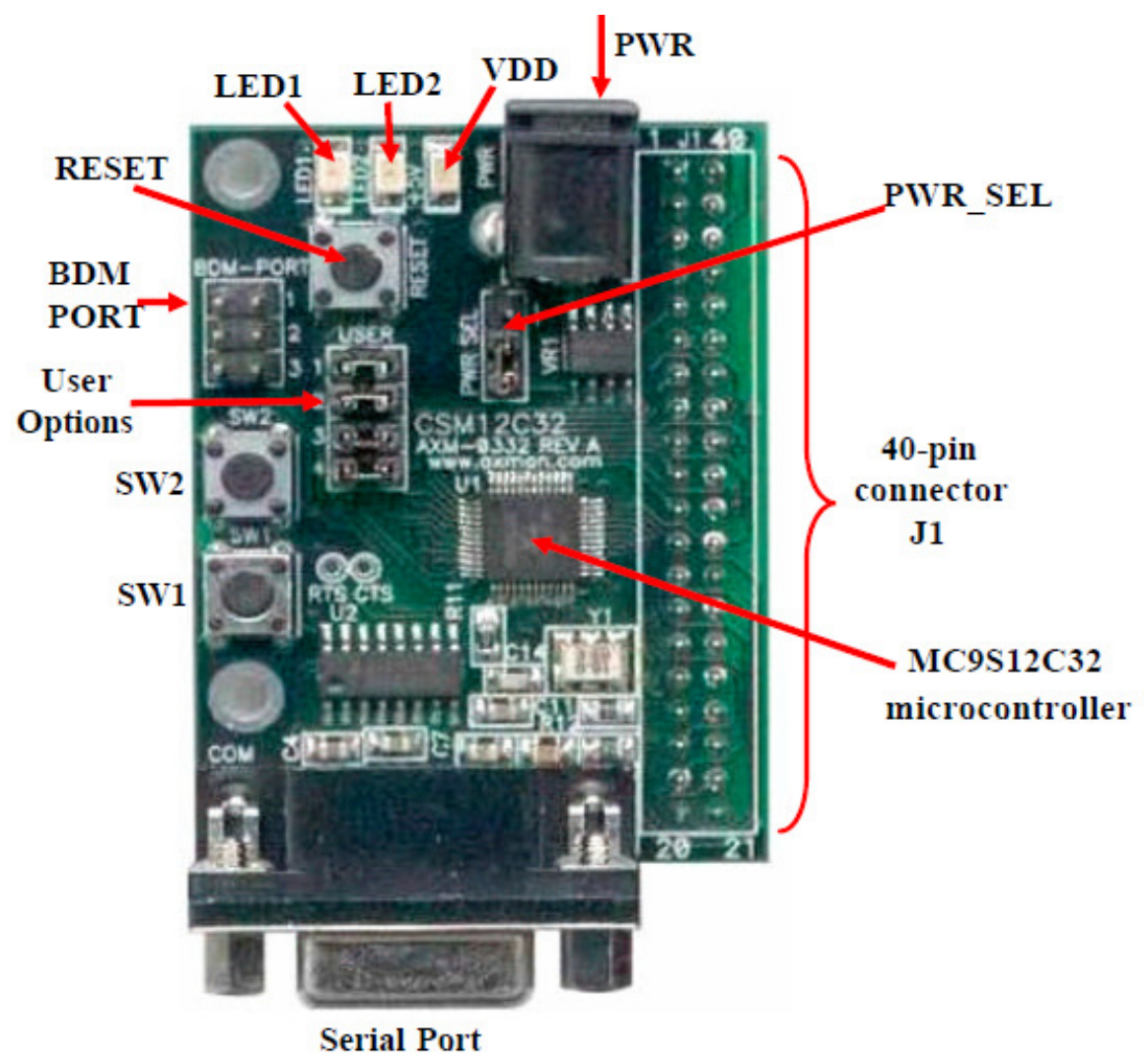

Figure 5.13 Diagram of the CSM-12C32 (Microcontroller module)

\section{Module Operation}

The MCU supports a default $38.4 \mathrm{~K}$ bps serial communication rate on the COM interface port within RS-232. Power is supplied to the CSM-12C32 module through the connector labeled PWR in the diagram Figure 5.13. The PWR_SEL jumper determines the source of input power.

The PWR connector allows the module to be powered from a transformer plugged into a standard wall outlet.

Connector J1 
Power can be supplied to the module through the pins J1-1 and J1-2. The range of voltage input is +3.3VDC - +5VDC (+/-5\%). The PWR_SEL option determines how power is routed to the module.

PWR_SEL Jumper

This is a 4-position option header which decides which way power is input to the module. See Table 5.1

Table 5.1 Power jumper connection

\begin{tabular}{|c|c|}
\hline Shunt Position & \multicolumn{1}{|c|}{ Power Option Selected } \\
\hline 1 & $\begin{array}{c}\text { Regulated +3.3VDC - +5VDC from } \\
\text { connector J1 }\end{array}$ \\
\hline 2 & $\begin{array}{l}\text { On-board regulator connected to PWR } \\
\text { connector }\end{array}$ \\
\hline $1 \& 2$ & $\begin{array}{l}\text { On-board regulator connected to PWR } \\
\text { connector and sourcing power through } \\
\mathrm{J} 1\end{array}$ \\
\hline
\end{tabular}

The shunt position 1 is used since we are receiving power through the $\mathrm{J} 1$ connector from the project board.

The LED indicators, LED1 and LED2, are connected to the MCU's I/O ports via the USER jumper bank. When the appropriate USER jumper is installed, the assigned LED is active. Each LED is active low (a zero written to it turns it on). MCU ports PA0 and PB4 drive LED1 and LED2, respectively. Two pushbutton switches SW1 and SW2 provide momentary active low input to the MCU. SW1 and SW2 are also connected to the MCU I/O ports by the option bank and provide input to MCU I/O ports PE0 and PP5 respectively.

The BDM port on the MCU shown in Figure 5.14 is a 6-pin header. It allows the connection of a background debugger for software development and debugging in realtime without using MCU I/O resources. This port is useful for programming the $\mathrm{MCU}$ while it's on the Power Simulator for real time testing and debugging without having to 
connect and disconnected the MCU for each time. Also, turning off and On the simulator to access the back panel in order to program and debug the MCUs can be very tedious.

\begin{tabular}{|c|c|c|c|}
\hline BKGD/MODC & 1 & 2 & \multirow{3}{*}{$\begin{array}{l}\text { GND } \\
\text { RESET } \\
+V_{\text {DD }}\end{array}$} \\
\hline $\mathrm{N} / \mathrm{C}$ & 3 & 4 & \\
\hline $\mathrm{N} / \mathrm{C}$ & 5 & 6 & \\
\hline
\end{tabular}

Figure 5.14 BDM I/O port

For testing and programing the MCU it is better to use $\mathrm{J} 1$ Connector I/O port signals shown in Figure 5.15 below:

\begin{tabular}{|c|c|c|c|}
\hline \multirow{2}{*}{$\begin{array}{r}\mathrm{V}_{\mathbf{x}} \\
\text { GND }\end{array}$} & 1 & 2 & \multirow{4}{*}{$\begin{array}{l}\text { PE1/IRQ } \\
\text { RESET }{ }^{*} \\
\text { MODC/BKGD } \\
\text { NC }\end{array}$} \\
\hline & 3 & 4 & \\
\hline \multirow{2}{*}{$\begin{array}{l}\text { PS1/TXD } \\
\text { PS0/RXD }\end{array}$} & 5 & 6 & \\
\hline & 7 & 8 & \\
\hline PP5/KWVP & 9 & 10 & $\mathrm{NC}$ \\
\hline \multirow{2}{*}{$\begin{array}{r}\mathrm{PE} 0 / \mathrm{XIRQ} * \\
\mathrm{PT} 0 / \mathrm{PW} 0 / \mathrm{IOC} 0^{*}\end{array}$} & 11 & 12 & $\mathrm{NC}$ \\
\hline & 13 & 14 & $\mathrm{NC}$ \\
\hline PT1/PW1/IOC1 & 15 & 16 & $\mathrm{NC}$ \\
\hline \multirow{2}{*}{$\begin{array}{l}\text { PM4/MOSI } \\
\text { PM2/MISO }\end{array}$} & 17 & 18 & PAD00/AN00 \\
\hline & 19 & 20 & PAD01/AN01 \\
\hline \multirow{3}{*}{$\begin{array}{r}\text { PM5/SCK } \\
\text { PM3/SS } \\
\text { PE4/ELCK }\end{array}$} & 21 & 22 & PB4 \\
\hline & 23 & 24 & PA0 \\
\hline & 25 & 26 & PM1/TXCAN \\
\hline PE7/XCLKS & 27 & 28 & PM0/RXCAN \\
\hline \multirow{2}{*}{$\begin{array}{l}\mathrm{PAD} 02 / \mathrm{AN} 02 \\
\mathrm{PAD} 03 / \mathrm{AN} 03\end{array}$} & 29 & 30 & PT2/PWV2/IOC2 \\
\hline & 31 & 32 & PT3/PV3/IOC3 \\
\hline \multirow{2}{*}{$\begin{array}{l}\text { PAD04/AN04 } \\
\text { PAD05/AN05 }\end{array}$} & 33 & 34 & PT4/PW04/IOC4 \\
\hline & 35 & 36 & $\begin{array}{l}\text { P15/10C5 } \\
\text { PT6/IOC6 }\end{array}$ \\
\hline \multirow{2}{*}{$\begin{array}{l}\text { PAD06/AN06 } \\
\text { PAD07/AN07 }\end{array}$} & 37 & 38 & PT7/IOC7 \\
\hline & 39 & 40 & \\
\hline
\end{tabular}

Figure 5.15 J1 connector I/O port

The programming and debugging through this port is done using the MCU project board shown in Figure 5.16. 


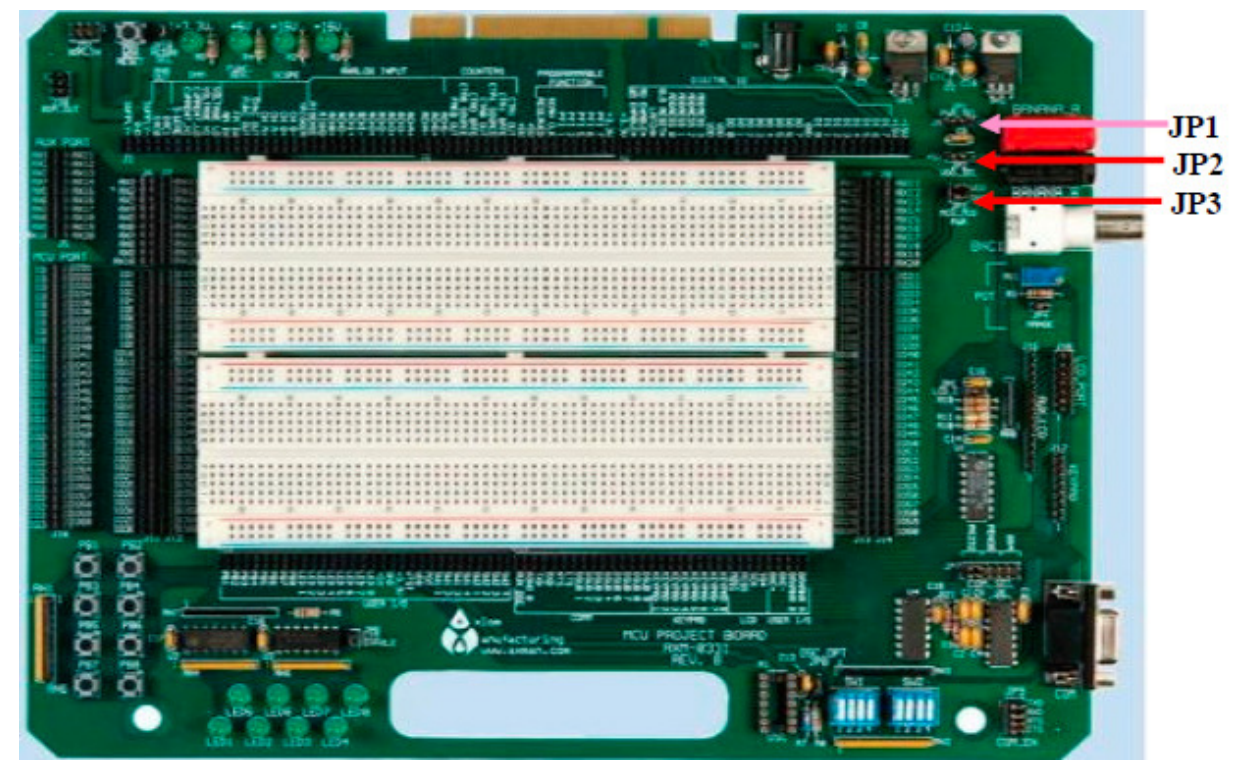

Figure 5.16 Debugging Board

\section{MCU Development Module (M522259DEMO):}

A more powerful MCU is used to program the agents, which is the M522259DEMO. This MCU consists of the M52259DEMOMCU and the M52259DEMOCOM boards connected via $0.5 \mathrm{~mm}$ stack connectors. The M52259DEMO allows the user to easily add functionality by simply matching the stack connector pin-out.

The M52259DEMOMCU board features the MCF52259 ColdFire microcontroller. An, open source, USB-BDM has been integrated to simplify application development and debug. A USB port with mini-AB USB connector is also provided. Four user LEDs and 2 user push buttons complete the M52259DEMOMCU board features. Additional MCU features are available at the stack connector see Figure 5.17. 


\section{M52259DEMOKIT Block Diagram}

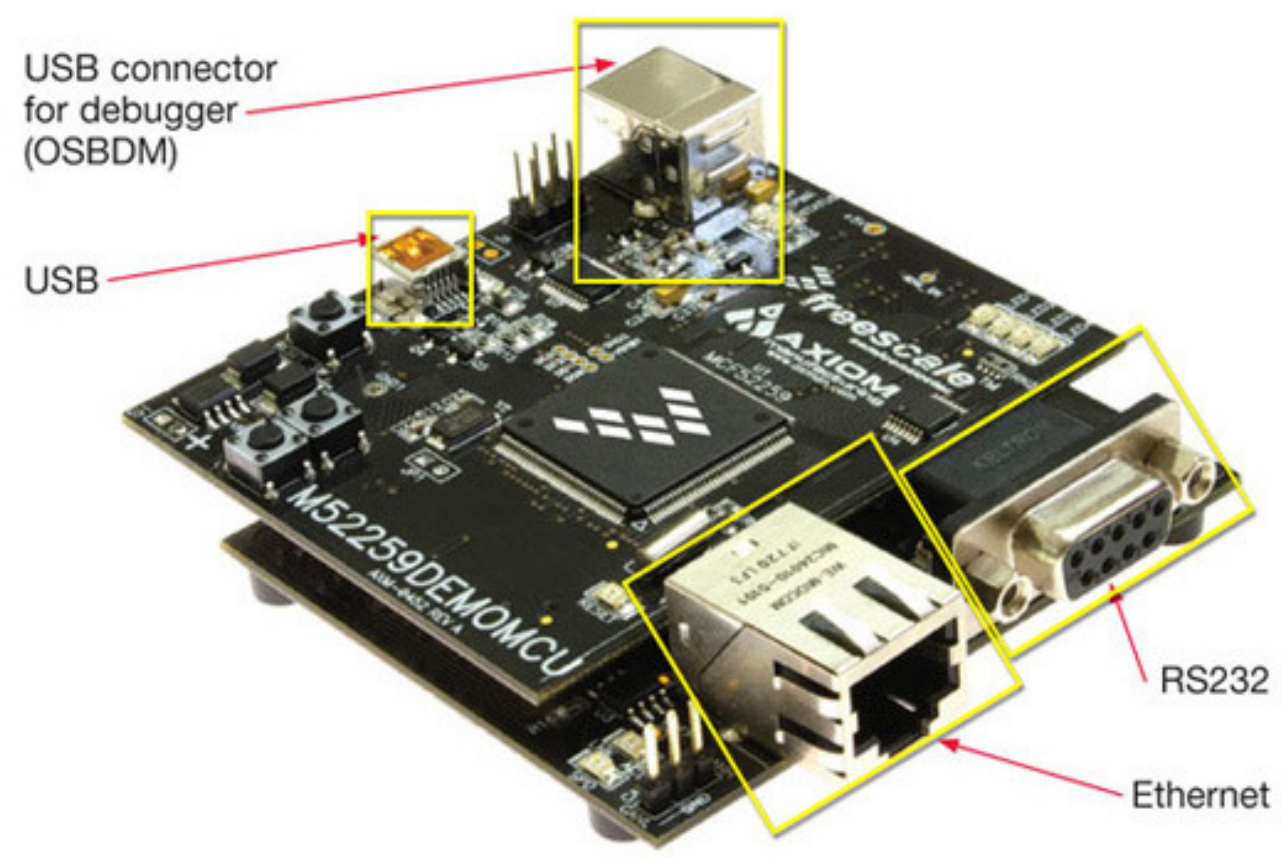

Figure 5.17 M522259DEMO board

The MCU has the following features:

- MCF52259 CPU, 144 LQFP

- V2 ColdFire Core w/ eMAC

- 80MHz internal bus

- 512 KB FLASH

- 64 KB SRAM

- 4ch, DMA Controller w/ Timers

- Fast Ethernet Controller (FEC)

- USB Physical Layer Interface (PHY)

- Mini-FlexBus External Bus Interface

- Fast A - D Converter

- FlexCAN 2.0B Module

- I2C, UART(s), QSPI,

- Integrated, Open-Source, USB BDM

- $48 \mathrm{MHz}$ XTAL

- miniAB USB Port

- RESET switch w/ indicator

- Power Input Selection Jumper 
- Power Input from USB BDM

- Power input from miniUSB

- Power Input from on-board regulator

- Power Input from terminal block (not installed)

- User Features

- 4 User LED's w/ enable

- 2 User Push Switches

- Connectors

- 0.5mm Stack Connectors for ease of expansion

- Type B USB connector

- miniAB USB Connector

- Supplied with DB9 Serial Cable, USB cable, Ethernet Cable, Support CD, and CodeWarrior Development

The IO Port connector consists of 2 stack connectors mounted on the bottom layer. These connectors allow the M52259DEMOCOM to be easily expanded by simply adding functionality. Figure 5.18 below shows the pin-out of connector $\mathrm{J} 1$.

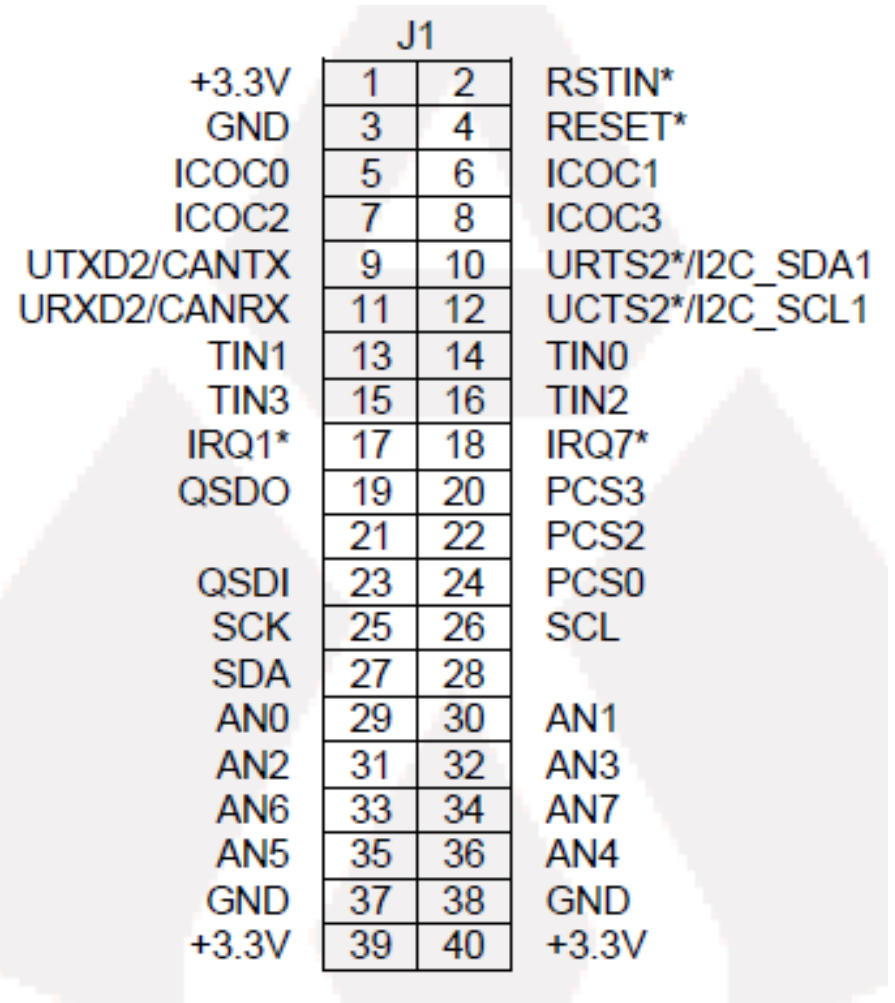

Figure 5.18 Connector J1 


\subsubsection{Processor Expert}

Processor Expert Code Warrior plug-in is designed for rapid application development of embedded applications for a wide range of microcontrollers and microprocessor systems.

The Processor Expert provides a more efficient and comfortable development environment integrated it as a plug-in to the CodeWarrior. The Processor Expert plug-in generates code from the Embedded Beans and CodeWarrior manages the project files and the compilation and debugging processes.

The key benefit of Embedded Beans is the same as using components in software design environments like Microsoft Visual Basic, where Embedded Beans provide hardware encapsulation in the form of a platform-independent standard.

Processor Expert has built-in knowledge (internal definitions) of the entire CPU with all of its units and integrated peripherals. The CPU units and peripherals are encapsulated into configurable components called Embedded Beans, each of which provides a set of useful properties, methods and events. An intuitive and powerful User Interface (UI) allows the user to define the system behavior in several steps. A simple system can be created just by selecting the necessary beans, setting their properties to the required values and maybe also dragging and dropping some of their methods to the user part of the project source code.

Advantages of using processor expert:

- In all phases of development, customers will experience substantial reductions in

- development cost

- development time

- Additional benefits in product development process are

- Integrated development environment increases productivity

- Minimized time to learn Freescale CPU

- Processor Expert User's manual Introduction

- Rapid prototyping of entire applications

- Modular and reusable functions

- Easy to modify and port implementations

The main task of Processor Expert is to manage CPU and other hardware resources and to allow virtual prototyping and design, code generation from beans, the ability to maintain user and generated code, and an event based structure significantly reduce the programming effort in comparison with classic tools. 
Chapter 5: Smart grid software and hardware development

\section{Embedded Beans}

Component (bean) is the essential encapsulation of functionality. For instance, the TimerInt bean shown in Figure 5.19 encapsulates all CPU resources that provide timing and hardware interrupts on the CPU.

\begin{tabular}{|c|c|c|c|c|c|}
\hline & Bean I & spector TI1:Timer & rint & & $-\square x$ \\
\hline & an Iter & s Visibility Help < & & Peript & heral Initialization > \\
\hline & operties & Methods | Events | & Comment & & \\
\hline$\checkmark$ & Bean! & & TI1 & & \\
\hline & Timer & & TMRA0_Compare & $\boldsymbol{I}$ & JMRA0_Compare \\
\hline$\checkmark$ & Count & & TMRAO & & TMRAO \\
\hline$\square$ & -InterI & jpt service/event & Enabled & & \\
\hline$\checkmark$ & Inter & & INT_TMRAO & & INT_TMRAO0 \\
\hline$\checkmark$ & Inter & upt priority & medium priority & - & 1 \\
\hline$\checkmark$ & Presc & & Auto selected pres & caler $\overline{-}$ & not set \\
\hline I & Interru & period & & $\ldots$ & Unassigned timing \\
\hline$\checkmark$ & Bean ! & ses entire timer & no & $D$ & \\
\hline$\Xi$ & -Initial & zation & & & \\
\hline$\checkmark$ & Ena & led in init. code & yes & $D$ & \\
\hline$\checkmark$ & Eve & ts enabled in init. & yes & $D$ & \\
\hline & ASIC & ADVANCED & PERT & & \\
\hline
\end{tabular}

Figure 5.19 TimerInt bean inspector

We can find many components called Embedded Beans in the Processor Expert Bean selector window. These components are selected to cover the most commonly required functionality used for microcontroller applications such as handling port bit operations, external interrupts, and timer modes, serial asynchronous/synchronous communications, A/D converter, I2C, CAN, etc.

A bean provides a clear interface. By setting properties, a user defines the future behavior of the bean in runtime. The user controls properties in design time by using the Beans Inspector. Runtime control of the bean function is done by the Methods.

Events are interfacing hardware or software events invoked by the bean to the user's code. This propriety is very suitable for designing agents based programs since the agent is supposed to be aware of its environment which means events that happen at the hardware side can be sensed by the agents for it to respond accordingly and in timely manner. Figure 5.20 below shows the CodeWarrior-Processor Expert interface. 


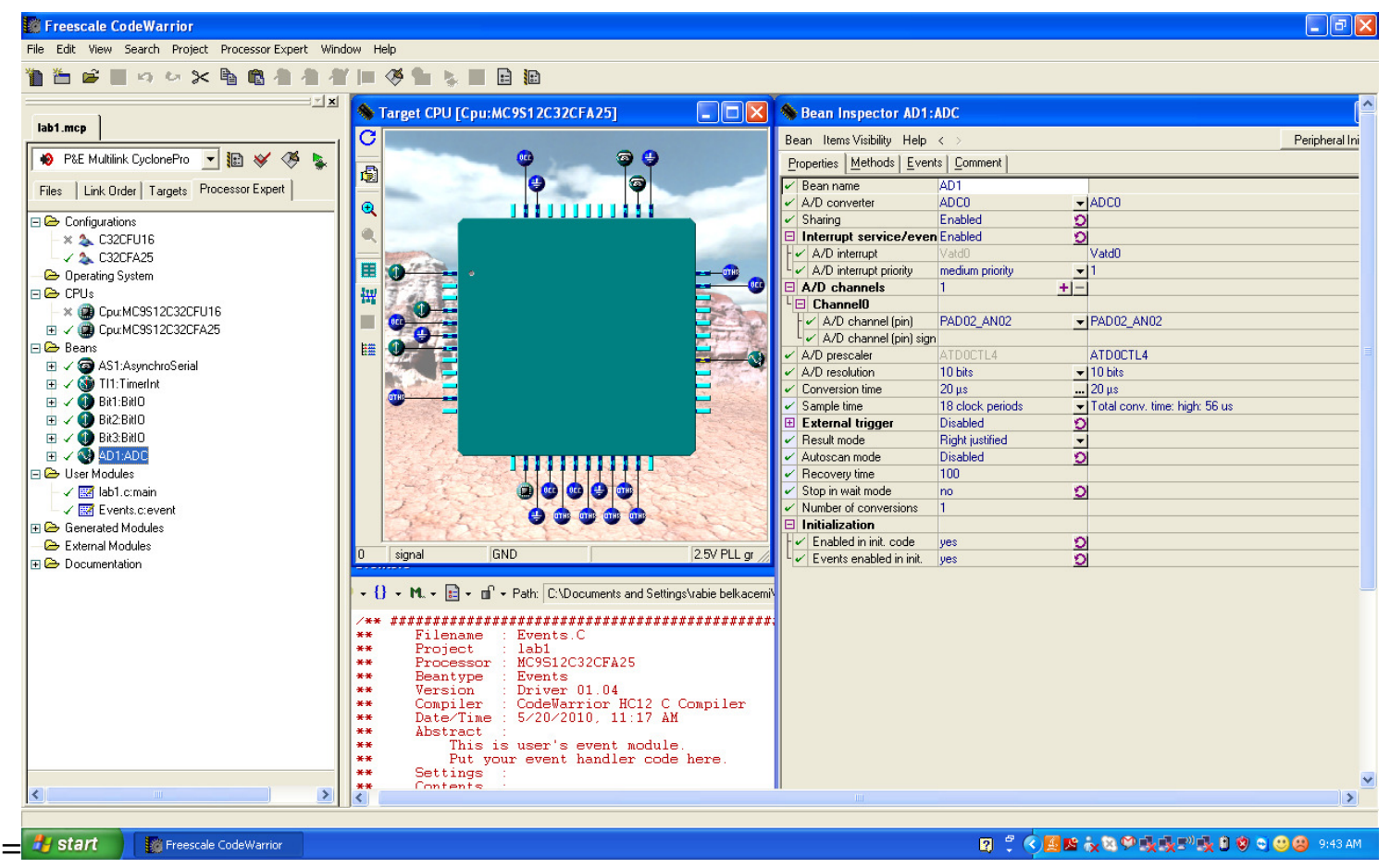

Figure 5.20 Processor expert interface

After hitting the debug button, a new window pops up showing real time debugging and execution of the program, Figure 5.21. 


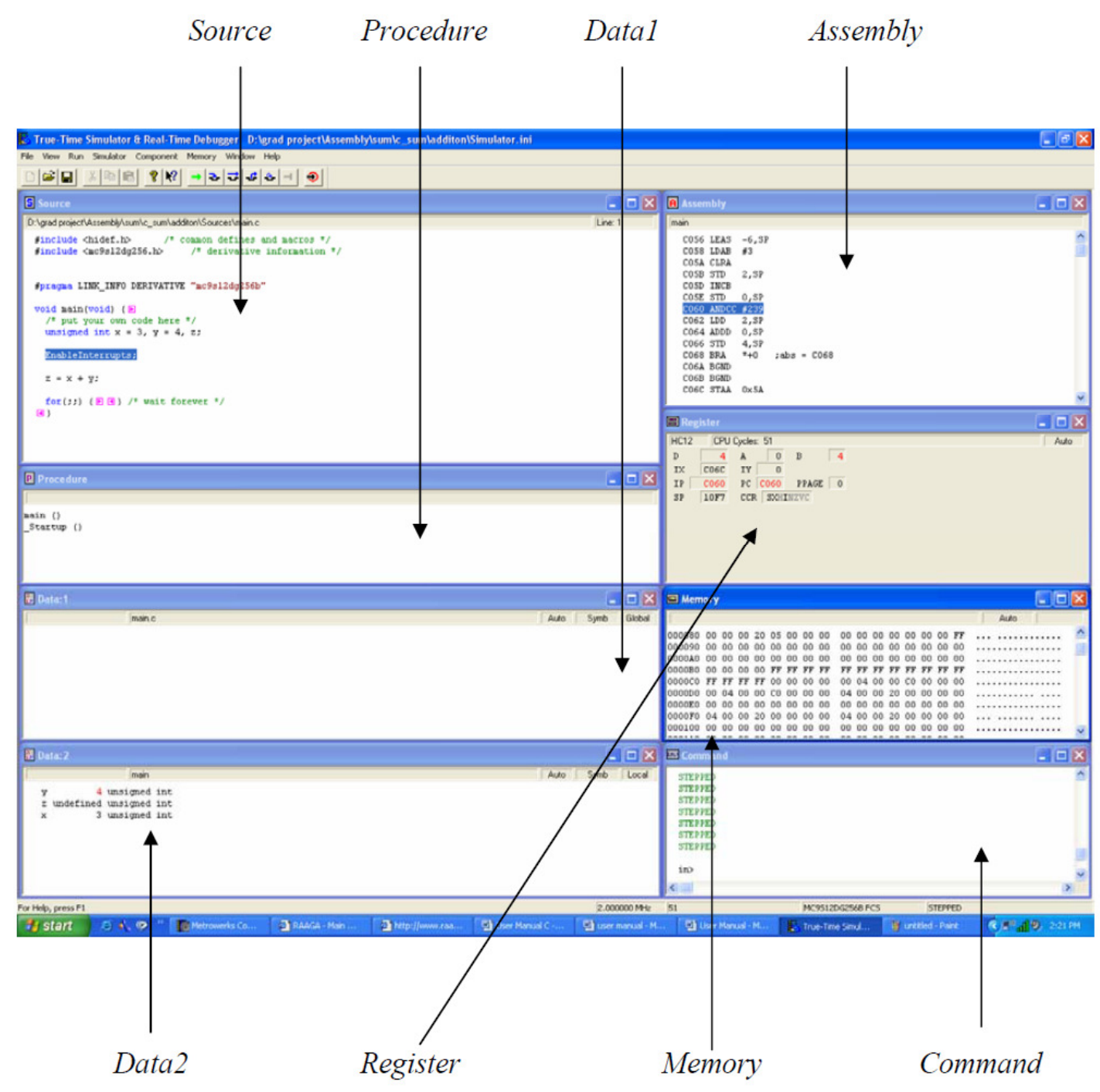

Figure 5.21 Real Time Debugger

\subsubsection{Current and voltage Sensors}

In this work, Closed Loop Hall Effect current transducers are used as sensors to feed the Analogue Digital Converter ADC of the MCU unit where the agent program resides.

Closed Loop Hall Effect sensing Features:

- Wide frequency range

- Good overall accuracy

- Fast response time 
- Low temperature drift

- Excellent linearity

- No insertion losses

Up until now the work is being performed using the LEM LA 25-NP which is a current sensor with a high rating but requires a bipolar power supply of $-15 /+15$. A large number of this component would require a separate power supply which is not practical for the size and complexity of the Power Simulator back panel. Because of that, in the second phase we are going to be using the LEM HY5 as sensor which is a unipolar transducer that requires $5 \mathrm{~V}$ power supply. In this conditions the MCU, Wireless Module, Sensors, and the phototriacs would have a common power supply.

On top of their robustness and their easy mounting, the LEM sensors are a better representation of the real world power systems components than the sensitive IC chips. Also, the output is directly fed to the MCU for conversion without the use of an SPI communication to pullout the data. The use of the SPI requires the use of the MQX which in its self a tedious and voids the use of Processor Expert for fast development.

The LEM devices used have the following Advantages:

- Excellent accuracy

- Very good linearity

- Low temperature drift

- Optimized response time

- Wide frequency bandwidth

- No insertion losses

- High immunity to external interference

- Current overload capability

The Figure 5.22 below show the LA25 NP mounted on the agent hardware. 


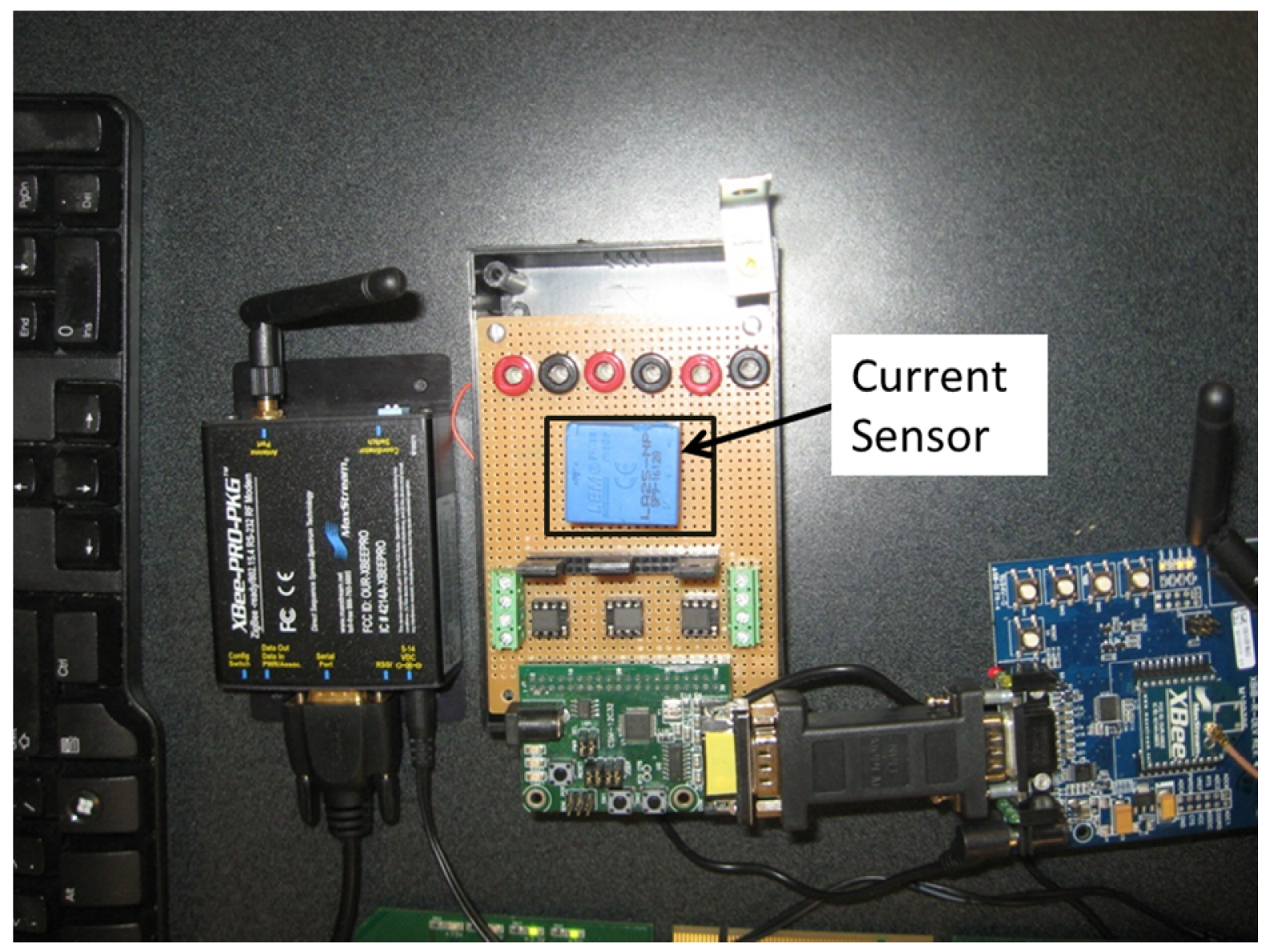

Figure 5.22 Sensor Hardware mounted on an agent module

\section{Electrical data and connections}

$\mathbf{I}_{\mathrm{PN}}$ Primary nominal r.m.s. current $25 \mathrm{At}$

$\mathbf{I}_{\mathrm{P}}$ Primary current, measuring range $0 \pm 36 \mathrm{At}$

$\mathbf{R}_{\mathrm{M}}$ Measuring resistance $\mathbf{R M} \min \mathbf{R M} \max$

with $\pm 15 \mathrm{~V} @ \pm 25$ At $\max 100 \Omega 320 \Omega @ \pm 36$ At max $100 \Omega 190 \Omega \square$

$\mathbf{I}_{\mathrm{SN}}$ Secondary nominal r.m.s. current $25 \mathrm{~mA}$

$\mathbf{K}_{\mathrm{N}}$ Conversion ratio 1-2-3-4-5 : 1000

In our case we choose the nominal value of $5 \mathrm{~A}$ by soldering the terminals of the sensor as shown in Figure 5.23. The measuring resistance $R_{M}$ is chosen to be $200 \Omega$ to have a maximum of $5 \mathrm{~V}$ voltage at the output of sensor in order to avoid exceeding the ADC's voltage rating.
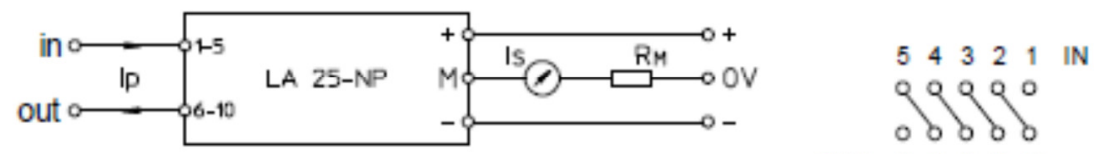

Figure 5.23 Sensor connections 


\section{Calibration}

The calibration depends on the resolution of the ADC. In case the of the 10bits resolution we can compute the measured current by the following formula:

$$
I=(\text { ADresult } \times 5) /\left(2^{10}-1\right)
$$

\section{Testing and validation of the measurements}

In the following section, the combined Sensor-MCU module is implemented on the power simulator to test if the measurements seen by the agents are correct. The Figure 5.24 below shows a snapshot of the display and the value measured at the agent hardware level.

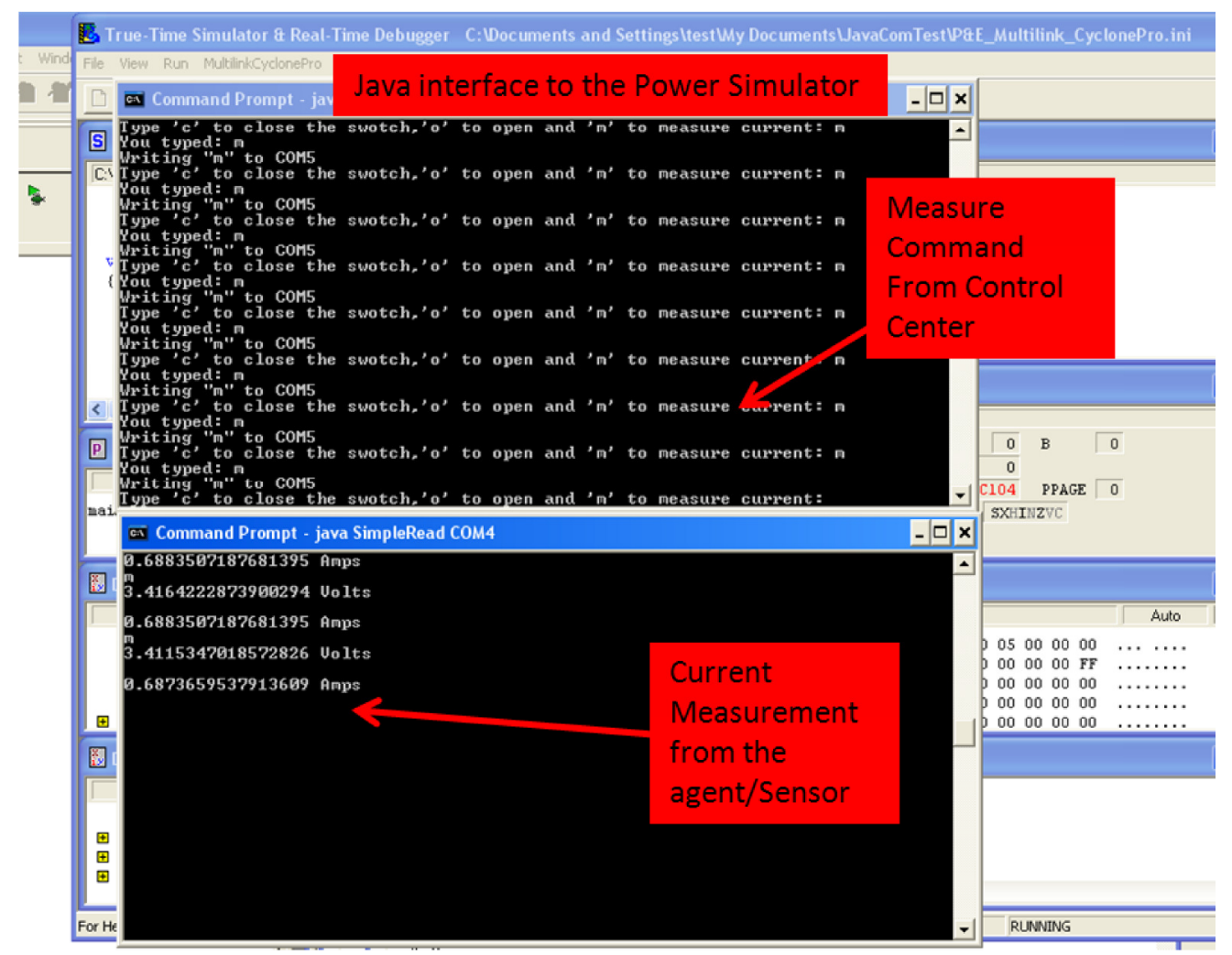

Figure 5.24 MCU-Sensor measurement display from the power simulator

The measurements recorded above are then compared with the power simulator imbedded Ampere Meter shown in Figure 5.25. We can observe that the two values are exactly marching after calibration. This approach of measurements is less complicated and easy to implement and calibrate than using IC based chips that uses an SPI communication to the MCU. 


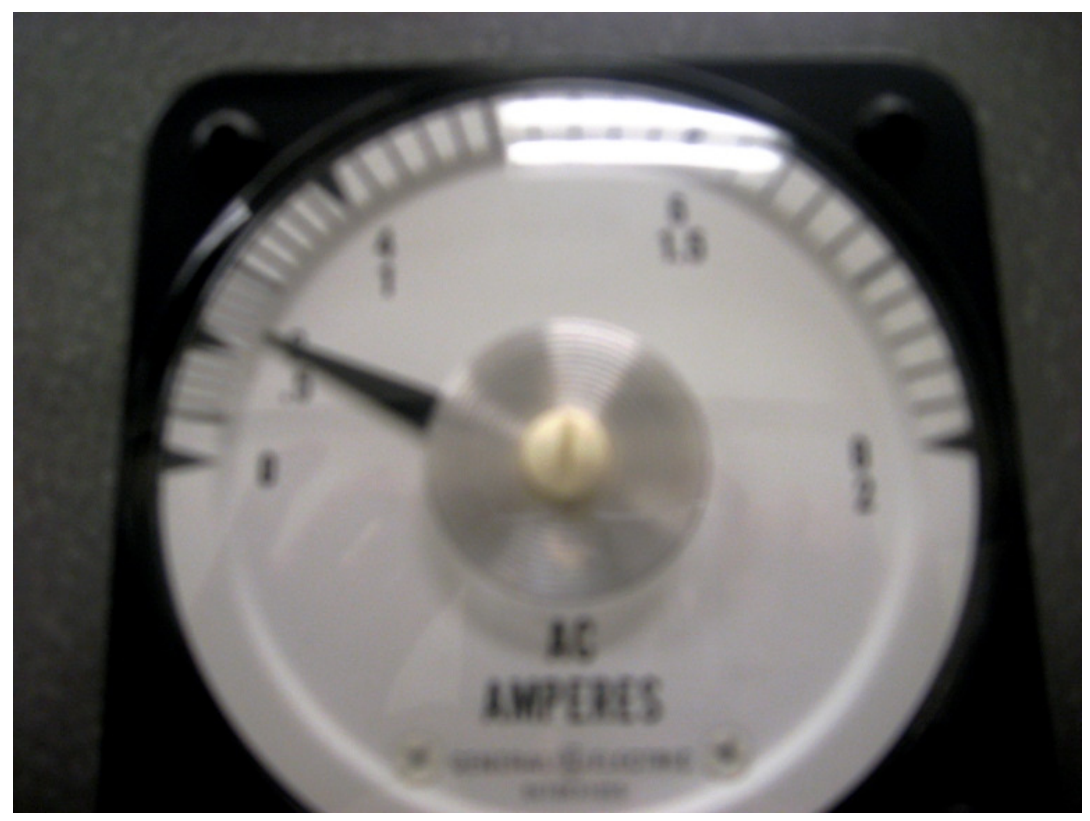

Figure 5.25 Power Simulator Ampere Meter

\subsubsection{Hardware Communication platform}

Integrated communications is identified as one of the key technologies to achieve a modern power grid. Due to its dependency on data acquisition, protection, and control, the modern grid cannot exist without an effective integrated communications infrastructure. Establishing these communications must be of highest priority since it is the first step in building the modern grid $[5]$.

The advances in wireless communication systems made it a very suitable technology to be used in the modern grid. In the WVSC project a wireless platform is expected to be implemented to support the communications between different components of the systems. For this reason, a wireless radio communication network platform is being designed and deployed on the Power Simulator. The network platform will offer a mean of communication to be used by the MAS not only for inter-Agent communications but also for Electric Devices-Agents communications for parameters setting or accessing data for example.

In this work, The XBee wireless transceiver (modem) using the IEEE 802.15.4 Low-Rate Wireless Personal Area Network protocol (LR-WPAN) for Wireless Sensor Networks (WSN) is used to develop a wireless network system, see Figure 5.26. This allows addressable communications between nodes. Data may be sent to individual nodes (point-to-point), or to all 
nodes in range (point-to-multipoint) using a broadcast address. Each Agent on the network is equipped with an Xbee module which allows it to communicate with other agents and devices.

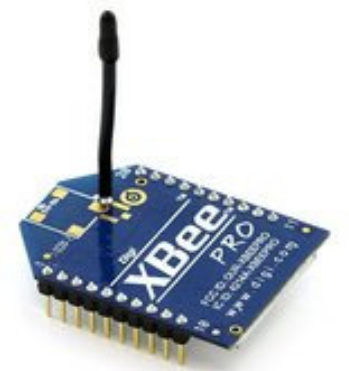

Figure 5.26 Xbee module

The Table 5.2 below shows the Xbee module features:

Table 5.2 Xbee module features

\begin{tabular}{|c|c|c|}
\hline & XBee & XBee-PRO \\
\hline Range - Indoor (sight/height dependent) & $100 \mathrm{ft}(30 \mathrm{~m})$ & $300 \mathrm{ft}(100 \mathrm{~m})$ \\
\hline Range - Outdoor (sight/height dependent) & $300 \mathrm{ft}(100 \mathrm{~m})$ & $1 \mathrm{mile}(1500 \mathrm{~m})$ \\
\hline Transmit Power & $0 \mathrm{dBm}(1 \mathrm{~mW})$ & $20 \mathrm{dBm}(100 \mathrm{~mW})$ \\
\hline Receiver Sensitivity & $-92 \mathrm{dBm}$ & $-100 \mathrm{dBm}$ \\
\hline TX Current & $45 \mathrm{~mA}$ & $214 \mathrm{~mA}$ \\
\hline RX Current & $50 \mathrm{~mA}$ & $55 \mathrm{~mA}$ \\
\hline Power-Down (Sleep) Current & $<10 \mathrm{uA}$ & $<10 \mathrm{uA}$ \\
\hline
\end{tabular}

Other important features and characteristics of the module are:

- IEEE 802.15.4 compliant, Low-Rate Personal Area Networking.

- 2.4 GHz DSSS (Direct Sequence Spread Spectrum).

-250,000 bits per second. 
- Acknowledgement and reties.

- Addressable, > 65,000 addresses available.

- Point-to-Point and Point-to-Multipoint (broadcast) messaging.

- Channel and Network ID selectable for cluster separation.

- Fully configurable via serial commands.

- Transparent transmission and reception.

- Receiver Strength indication.

- Free X-CTU interface software.

- Free and unlimited technical from MaxStream.

\section{Point-to-point communication}

Each node on the network is identified by a unique 16-bit address allowing over 65,000 devices on a single network. As illustrated Figure 5.27, a device at address 0 may communicate with various addresses, or device node 1 may communicate directly with another node. This forms a point-to-point network where anyone node may communicate with another (range dependent),.

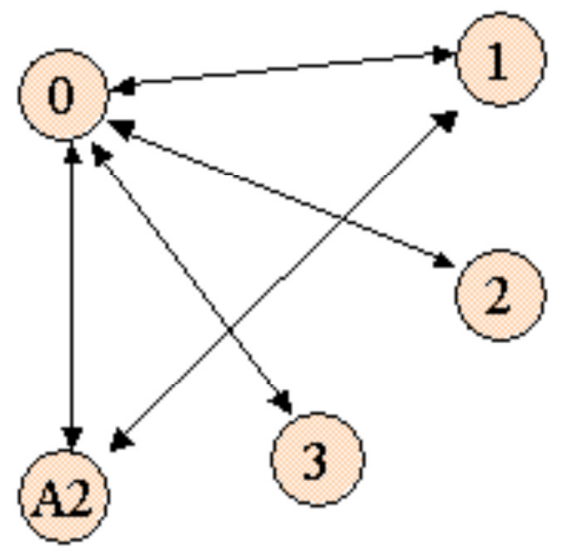

Figure 5.27 Communication network

The XBee units have numerous settings for configuration, two of which are:

MY - The node's own address (16-bit)

DL - Destination Low (16-bit)

By configuring these, a node is given an address, and a node to communicate to. For example, for node 0 to send data to node 1 :

$\mathbf{M Y}=\mathbf{0}$ 
DL $=1$

The data will be delivered with error checking, an acknowledgement, and up to 3 retries if needed. For node 2 to send data to node 0 :

$\mathrm{MY}=\mathbf{2}$

DL $=0$

Node 1 may also send data to node A2 (hexadecimal) by setting:

$\mathbf{D L}=\mathbf{A 2}$

\section{Point-to-Multipoint Broadcast Messages}

A broadcast message is one sent from a node to all listening nodes. This is performed without acknowledgements or retries. To send a broadcast message, DL is set to FFFF. This is useful if we wish to poll all devices or send a control message to all. When devices send data they use clear channel assessment (CCA) to help ensure they do not talk over one another.

\section{Communication network for MASs}

In the case of a MAS system we can find a mixture of point-to-point communication such interzonal agent communication and Point-to-Multipoint Broadcast communication such as substation/control center-zonal agent communication, see Figure 5.28.

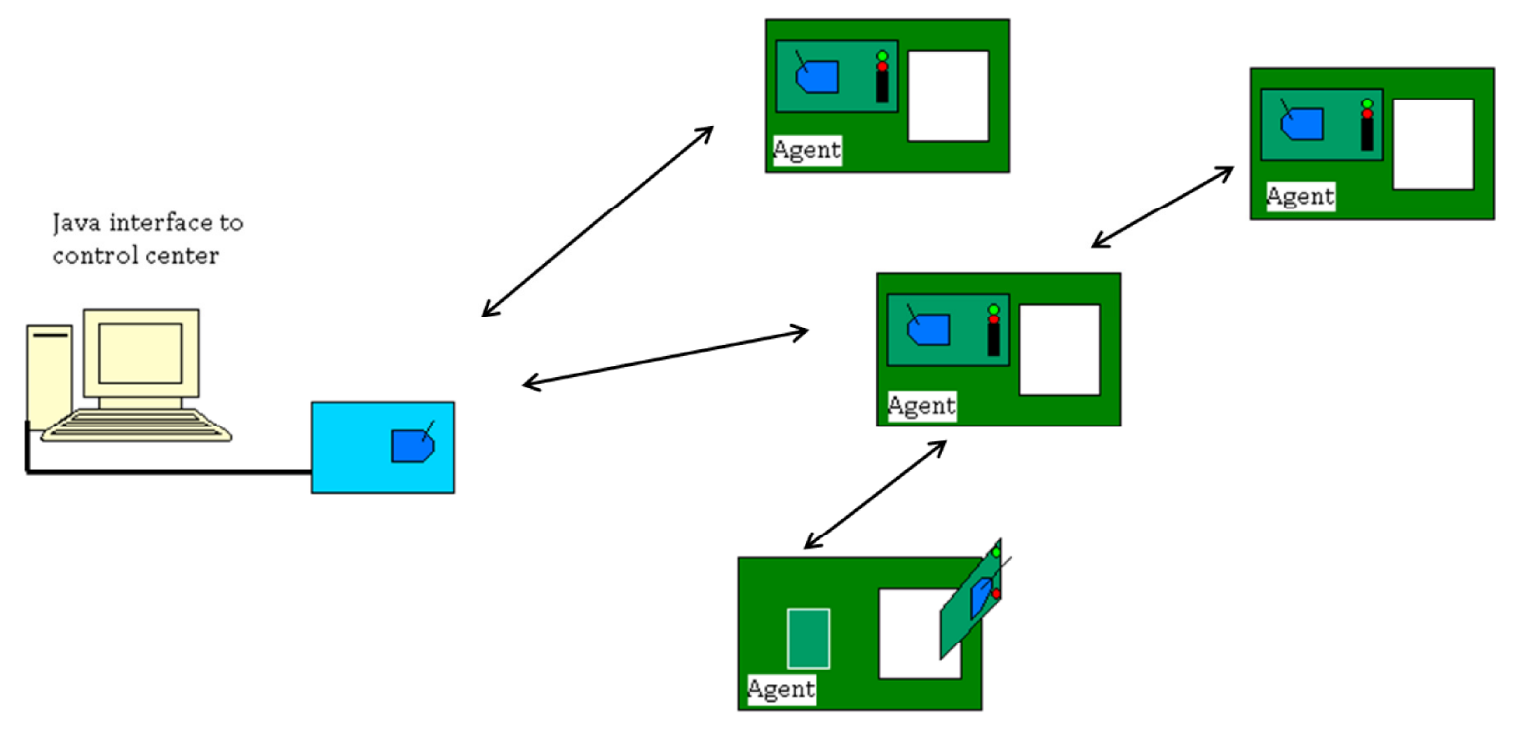

Figure 5.28 Communication Network of MAS for Power systems

\section{Configuring the XBee from X-CTU Software}

The module's address may be changed through either the X-CTU software Modem Configuration tab or using AT commands from a terminal window, or serially from a device. Figure 5.29 shows the configuration from the X-CTU software using the USB2SER adapter. Once the device is read 
(READ button), settings are modified and new settings downloaded using the WRITE button. Through this software all the setting such as baud rates and communication modes can be changed.

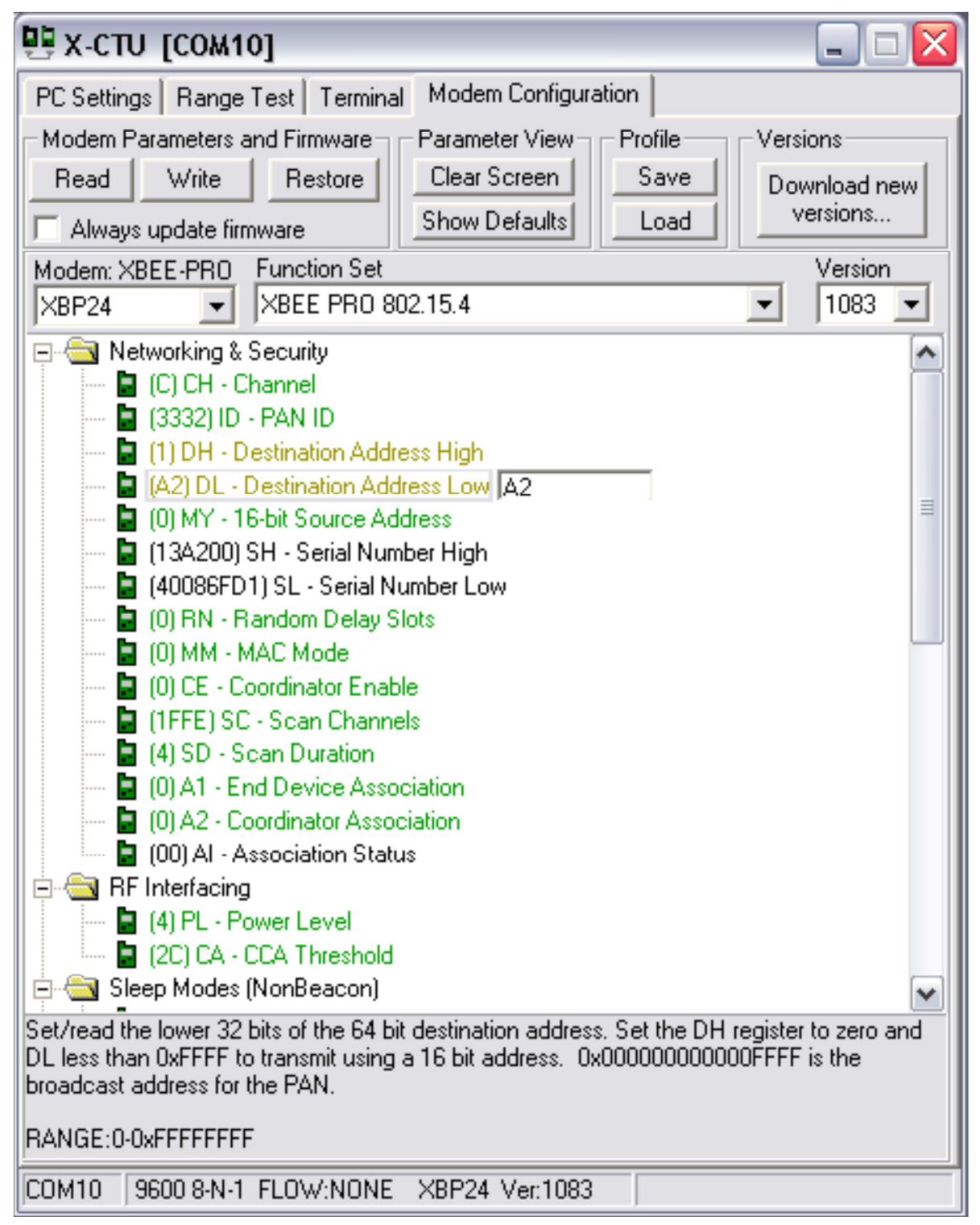

Figure 5.29 MaxStream's X-CTU Modem Configuration Software

\section{Configuring the XBee from Terminal}

Figure 5.30 shows the configuration using a terminal window set for $9600,8-\mathrm{N}-1$. The device is placed in command mode by issuing a "+++". To ensure data that contains this does not shift the unit into command mode, a guard time before and after is required - that is, no other data can be 
sent for a time period before or after the +++ , this includes carriage returns. Once in command mode, AT commands are sent to configure the unit. ATCN is used to exit command mode. Sending the command with no value will cause the unit to return the current value or setting as is shown. In this example, once in command mode the values of DL and MY are requested, then modified, then requested once again. Command mode is exited and data is sent between terminals.

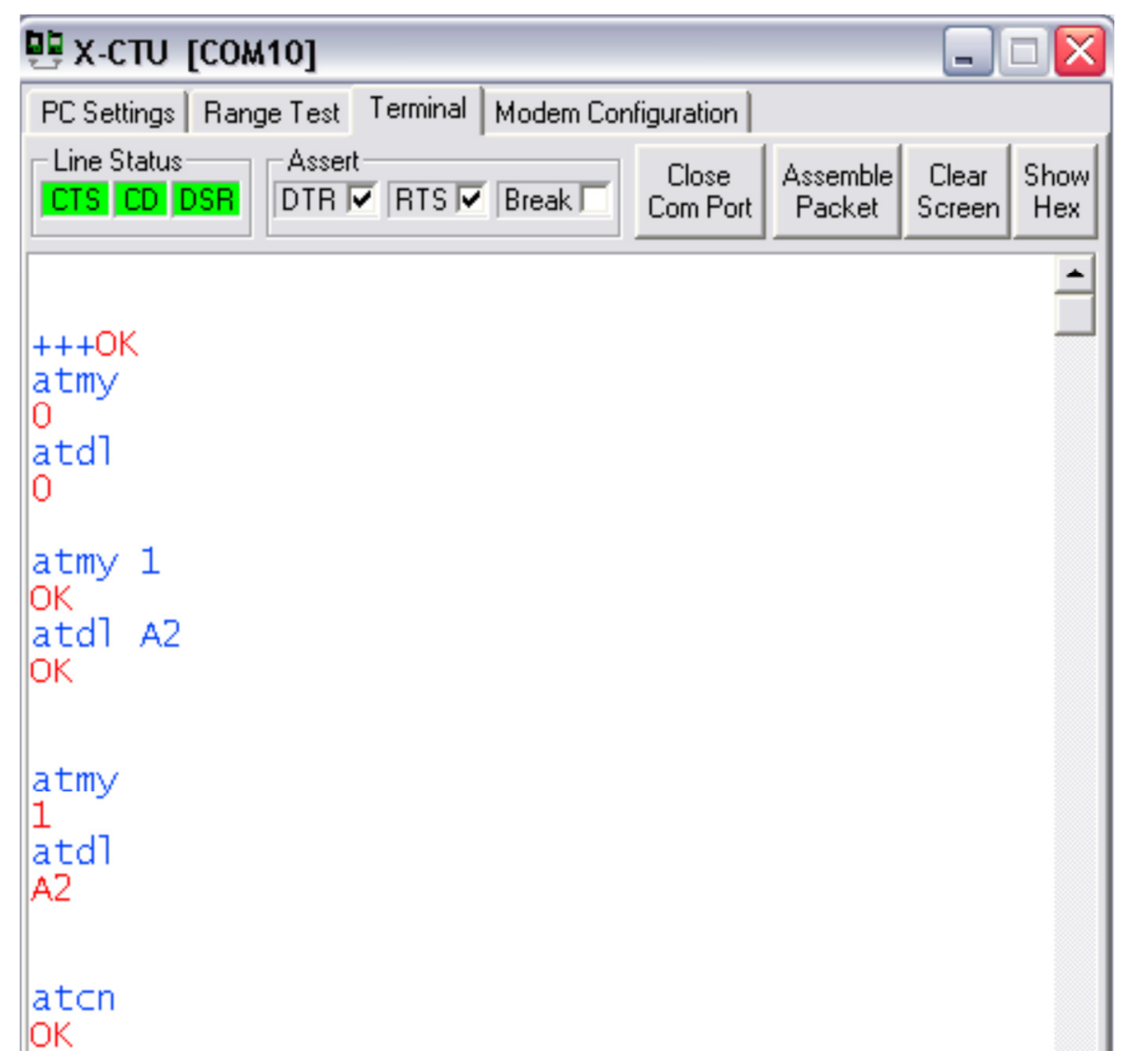

Figure 5.30 Programming the Xbee module through X-CTU software

Even though the X-CTU software is used for the example, HyperTerminal® or any terminal program running at 9600 Baud, 8-N-1, may be used. Data may be sent and received through the terminal window as well. In real time applications such as agent systems, this configuration is performed by the agent itself. For example, if Agent A1 wants to talk to Agent A2 it must first send an AT commands to the module to program the destination address to be A2 only then the message can be sent to A2.

\section{Summary of Common Configuration Commands}

A summary of common configuration commands is listed in Table 5.3. 
Table 5.3 Xbee configuration commands

\begin{tabular}{|c|c|}
\hline Command & Description \\
\hline MY & $\begin{array}{l}\text { Sets/ Read the current node address. Address are in hexadecimal, 0-FFFE } \\
\text { ATMY } \\
\text { ATMY A2 }\end{array}$ \\
\hline DL & $\begin{array}{l}\text { Sets/ Read the destination node address. Addresses are in hexadecimal, 0-FFFE } \\
\text { ATDL } \\
\text { ATDL } 1 \\
\text { Use FFFF to broadcast to all nodes. }\end{array}$ \\
\hline CH & $\begin{array}{l}\text { Sets/Reads the current RF channel in the } 2.4 \mathrm{GHz} \text { range. This can be used for frequency } \\
\text { separation between different } 802.15 .4 \text { networks or to limit interference from other } \\
2.4 \mathrm{GHz} \text { devices. } \\
\text { ATCH } \\
\text { ATCH }\end{array}$ \\
\hline ED & $\begin{array}{l}\text { Energy Detect - Performs a scan of background energy. Useful in deciding on a channel to use. Use } \\
\text { from the terminal window. }\end{array}$ \\
\hline ID & $\begin{array}{l}\text { Sets/Reads the current Personal Area Network ID (PAN ID). Allows network separation } \\
\text { between devices in same location. } \\
\text { ATID } \\
\text { ATID } 3350\end{array}$ \\
\hline NI & $\begin{array}{l}\text { Sets/Reads the node descriptive identification, } 20 \text { characters. } \\
\text { ATNI } \\
\text { ATNI Temperature Sensor }\end{array}$ \\
\hline BD & $\begin{array}{l}\text { Sets/Reads the value for the current serial baud rate. } \\
0-7 \text { (standard baud rates) } 0=1200 \text { bps } 1=24002=48003=9600 \\
4=192005=384006=576007=115200 \\
\text { ATBD } 2\end{array}$ \\
\hline SM & $\begin{array}{l}\text { Sets/Reads the sleep mode of the XBee. While sleeping, power consumption is <10uA. } \\
\text { When set to } 1 \text {, the SLP_R input of the AppBee Mod board may be used to place the } \\
\text { device in an idle state. } \\
\text { ATSM } \\
\text { ATSM } 1\end{array}$ \\
\hline WR & $\begin{array}{l}\text { Writes the current configuration to XBee non-volatile memory so that on power up the settings will } \\
\text { persist. } \\
\text { ATWR }\end{array}$ \\
\hline $\mathbf{R E}$ & $\begin{array}{l}\text { Restores the default settings of the XBee. } \\
\text { ATRE }\end{array}$ \\
\hline $\mathbf{C N}$ & $\begin{array}{l}\text { Exits command mode. A timeout period will also exit from command mode. } \\
\text { ATCN }\end{array}$ \\
\hline
\end{tabular}

\section{Testing wireless communication}


In the following section we are testing the communication network consisting of three nodes. The first module is configured in broadcast mode for data collection, and the two the other modules represent two nodes or agents on the circuit, set up shown in Figure 5.31 below.
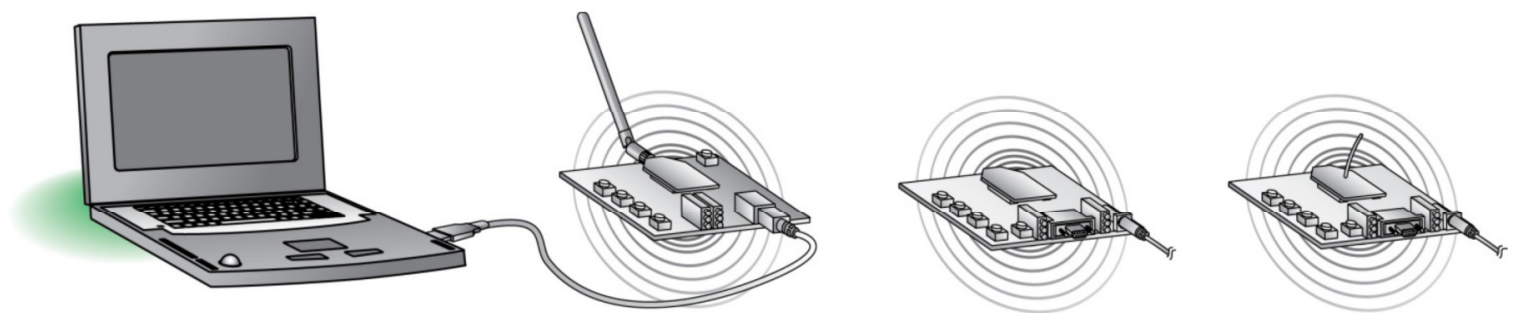

Figure 5.31 Hardware set up

In Figure 5.32, we are showing the experimental setup where the different nodes are highlighted.

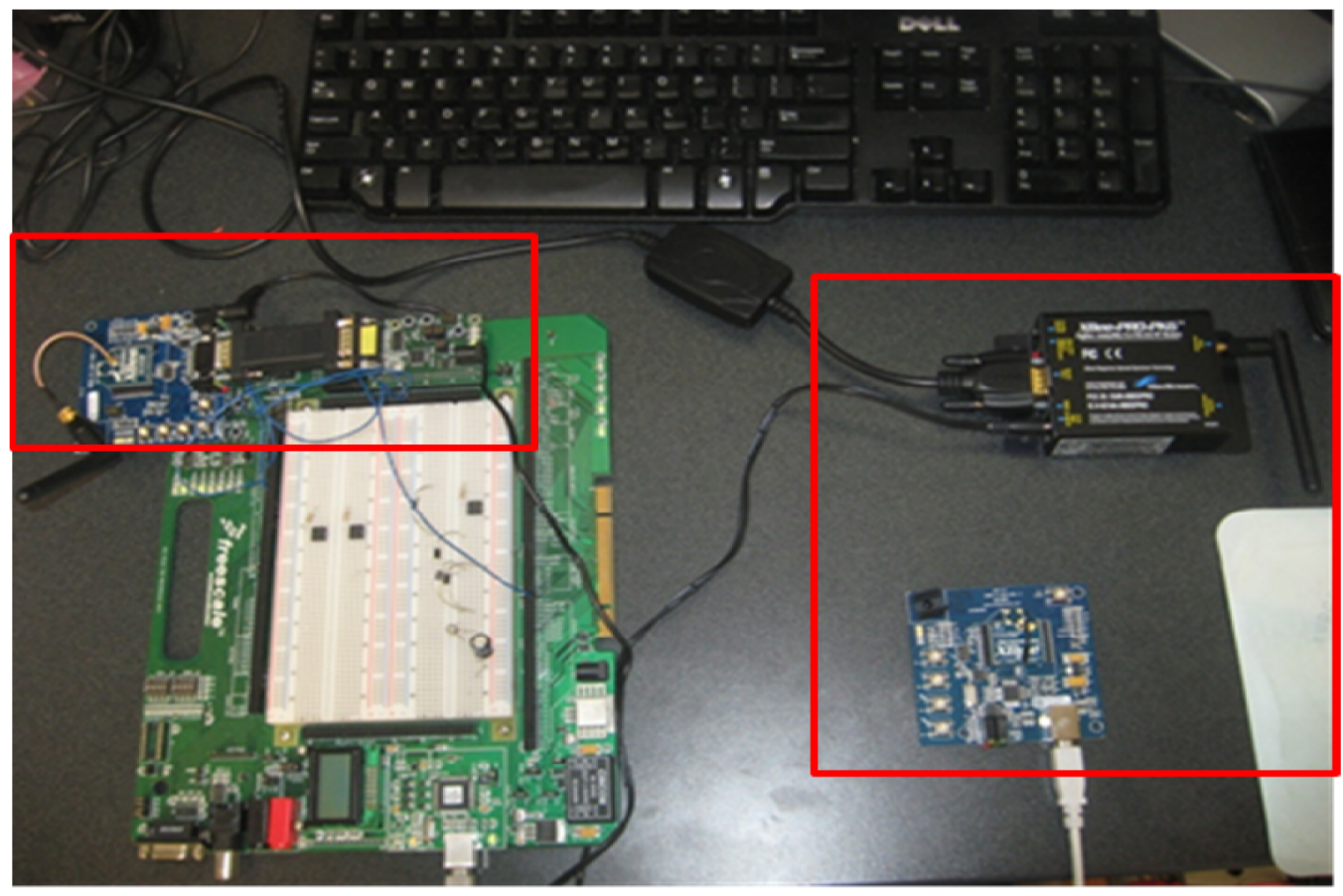

Figure 5.32 Hardware communication test set up

In this experiment, node/Agent $\mathrm{A}$ sends a request to node/Agent $\mathrm{B}$ for current measurements at its location. After the request is received by AgentB a 10bit format value of the current is sent back to the Agent B. Then, a second action request of closing the switch is sent by Agent A to AgentB. After receiving the request Agent $\mathrm{B}$ output a $5 \mathrm{~V}$ signal through its pin. In Figure 5.33 we are showing the communication messages. 


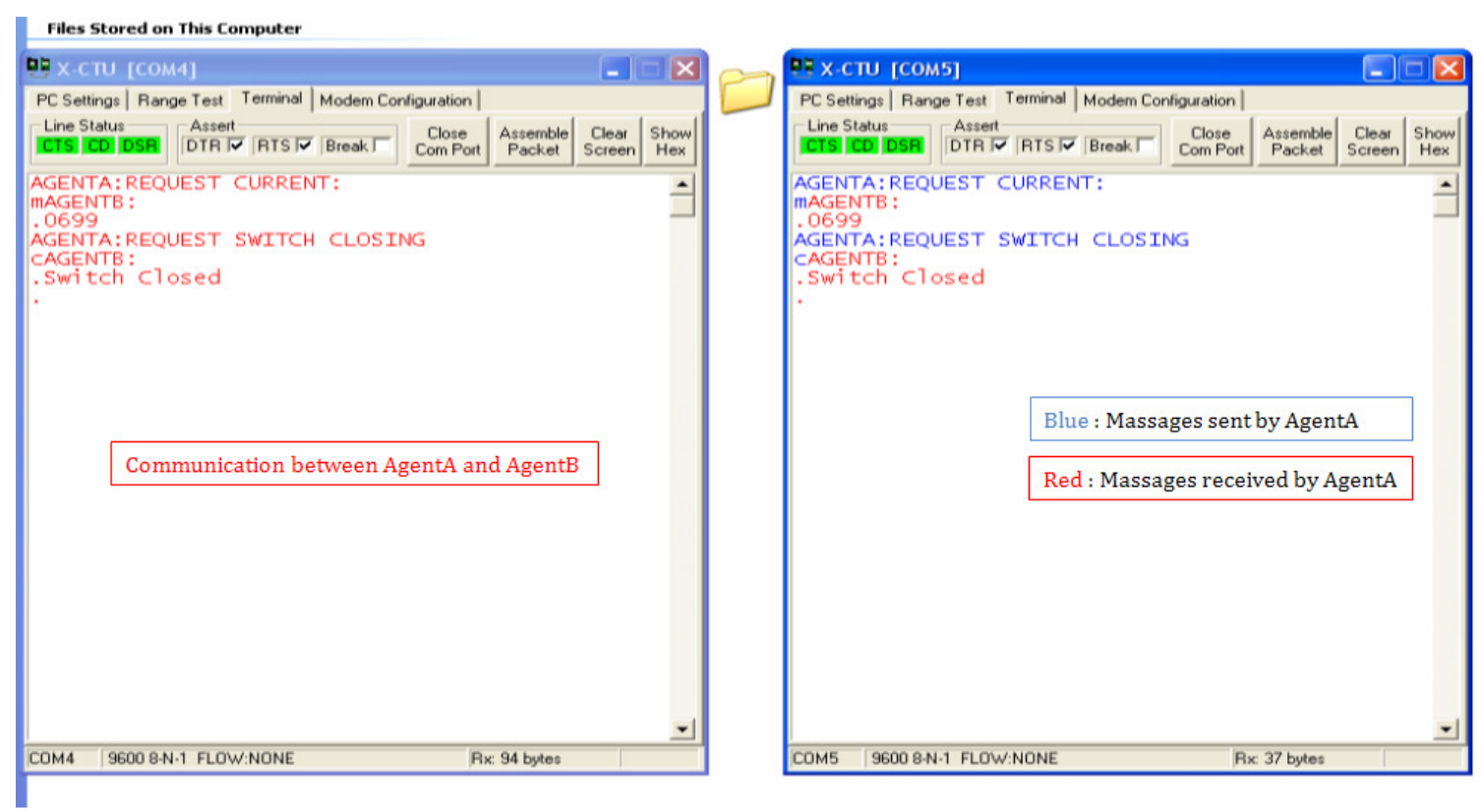

Figure 5.33 Hardware communication test

\subsubsection{Electronic switches design and control}

\section{General Description}

In this work the BTA208x Triac is chosen to design the three phase electronic switch. The BTA208X-1000B is a high voltage, high commutation triac in a full pack, plastic envelope. This triac is intended for use in motor control circuits where high blocking voltage, high static and dynamic $\mathrm{dV} / \mathrm{dt}$ and high $\mathrm{dI} / \mathrm{dt}$ can occur. This device can commutate the full rated rms current at the maximum rated junction temperature, without the aid of a snubber.

To drive the triacs we are using the PC4SD11NTZ Series Phototriac Coupler that includes an infrared emitting diode (IRED) optically coupled to an output Phototriac. These devices feature full wave control and are ideal isolated drivers for medium to high current Triacs. DIP package provides $5.0 \mathrm{kV}$ isolation from input to output with superior commutative noise immunity see Figure 5.34. 


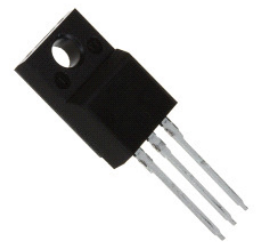

(a)

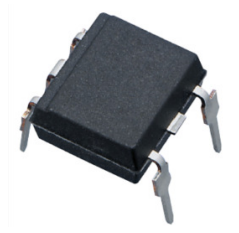

(b)

Figure 5.34(a) BTA208X-1000B (b) PC4SD11NTZ

In Figure 5.35 below, we are showing the electrical circuit driving the Triacs.

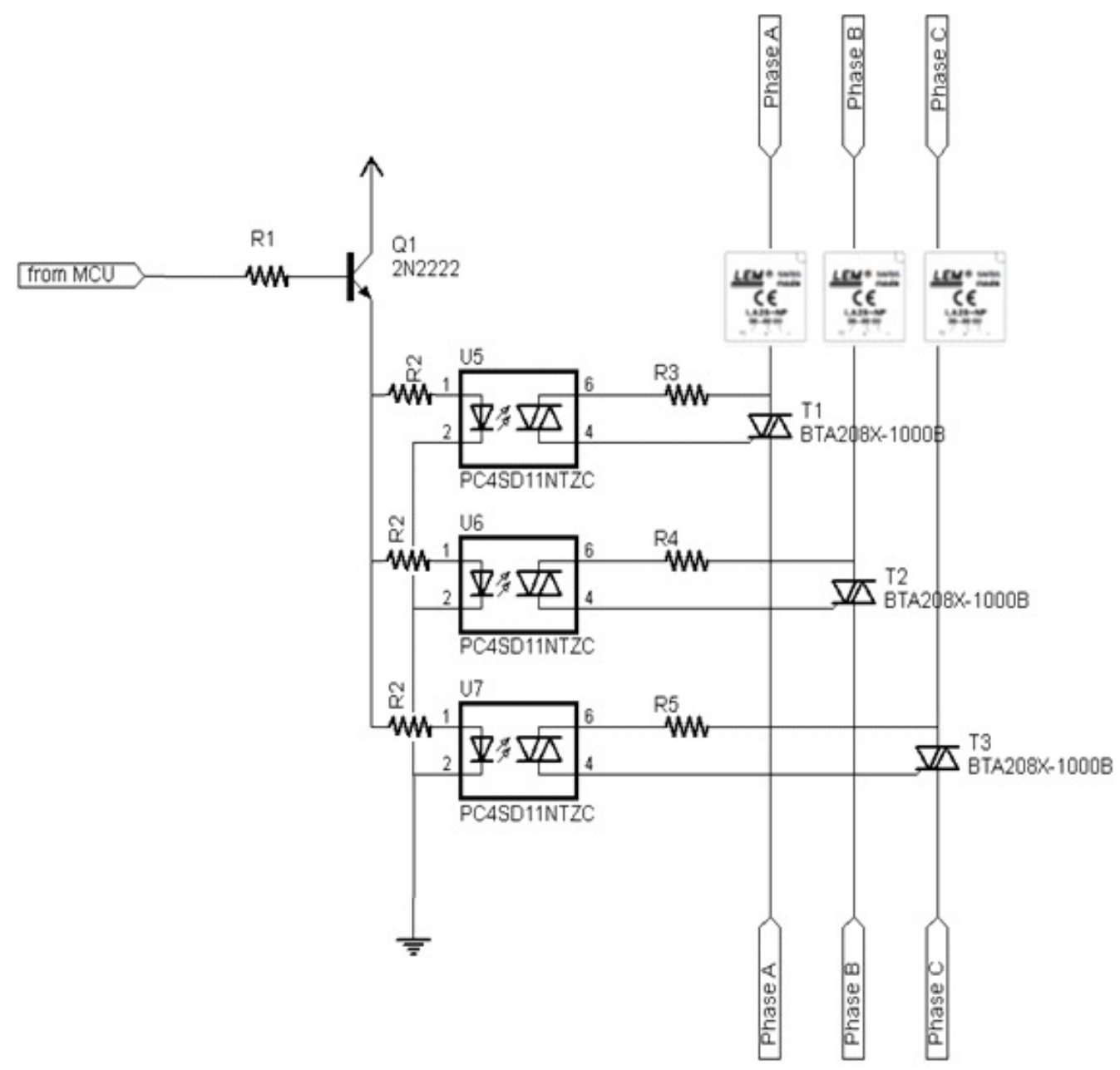

Figure 5.35 Triac triggering circuit 
The hardware implementation of circuit is shown in Figure 5.36.

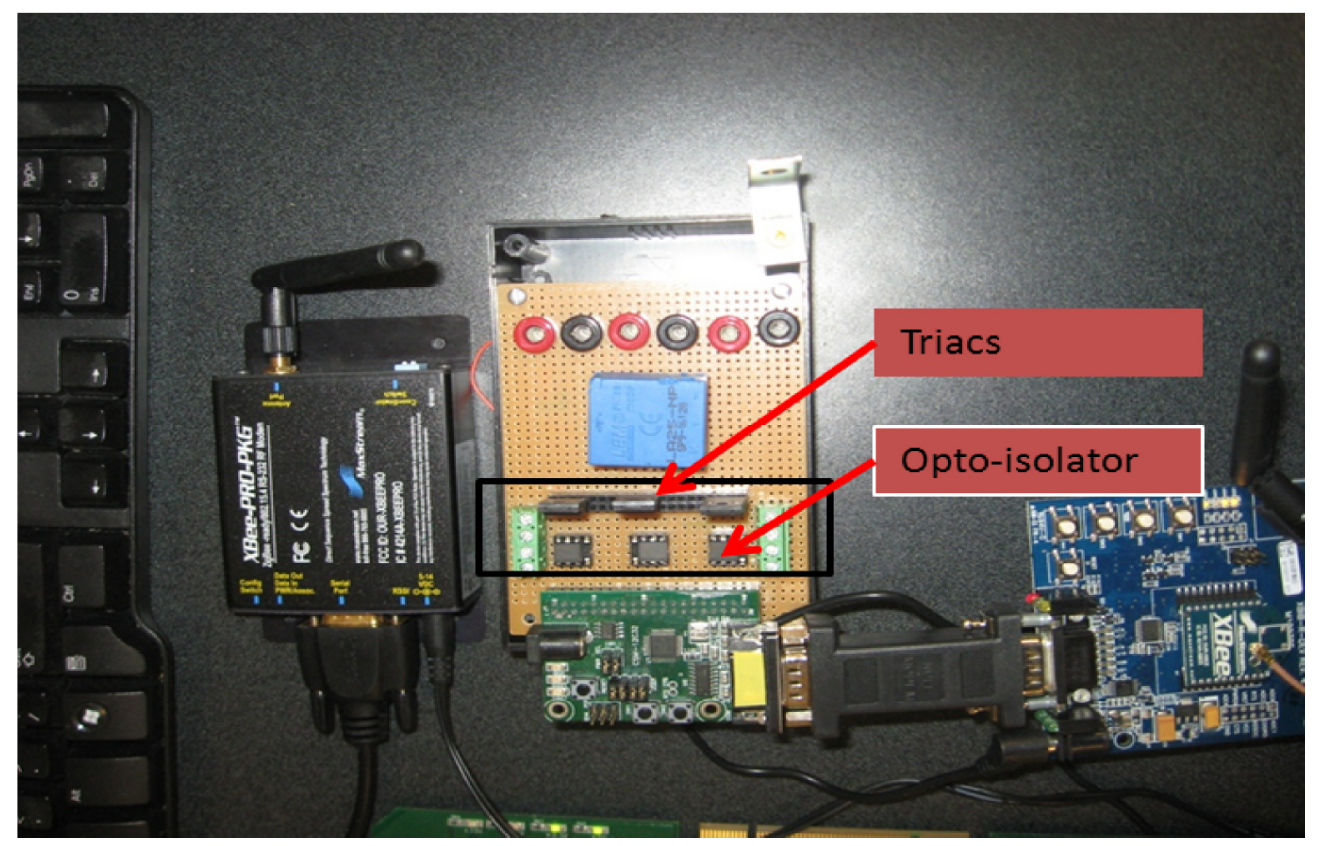

Figure 5.36 Hardware showing the assembled phototriacs-triacs

In designing the circuit the resistor $R_{1}$ is used as a current limiter and is chosen to be a round $10 \Omega . R_{2}$ is designed by performing tests and calibration to meet the nominal value of the current on the DC side of the phototriac device see Figure 5.37. In this case $R_{2}$ is found to be $150 \Omega$.

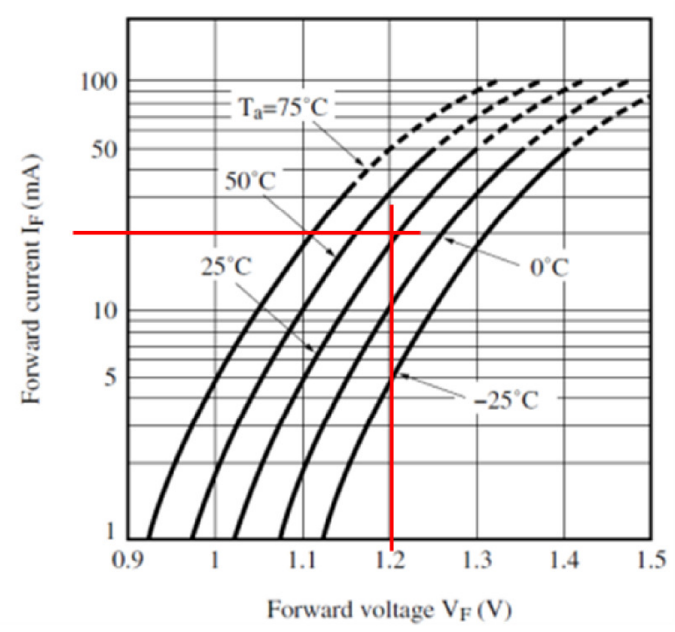

Figure 5.37 Phototriac PC4SD11NTZ characteristic 
The maximum surge current through the phototriac is determined by the maximum load voltage and the value of the resistors $R_{3,4,5}$. If the maximum surge current of the phototriac IFP is $1.2 \mathrm{~A}$ and we assume a $750 \mathrm{VAC}$ on the Power Simulator line, the value of $R_{3,4,5}$ can be determined as follows:

$$
R_{3,4,5}=\frac{V_{\text {inm }, a x}}{I_{F P}}=\frac{750}{1.2}=625 \Omega
$$

\section{Recloser Design}

The recloser is designed by programming the MCU to act and control the electronic switch to open and close depending on the fault current measurement. The switching cycle of the recloser can be programmed and set to the desired value (usually chosen to be equal to the current cycle).

\subsubsection{Integration and Testing on the Power Simulator}

In the following section, we are putting together all the hardware presented previously and integrated into the Power Simulator for testing.

In the Figure 5.38, Figure 5.39, and Figure 5.40 below, four complete hardware agent prototypes implemented on the Power Simulator back panel are shown.

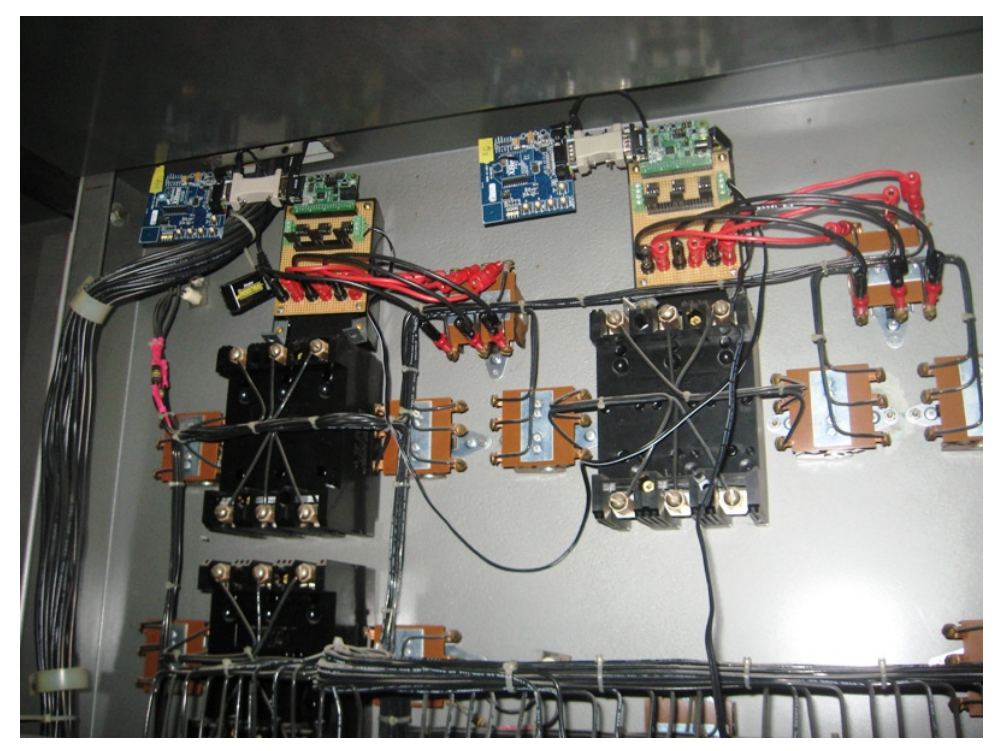

Figure 5.38 Agent prototype on Simulator 


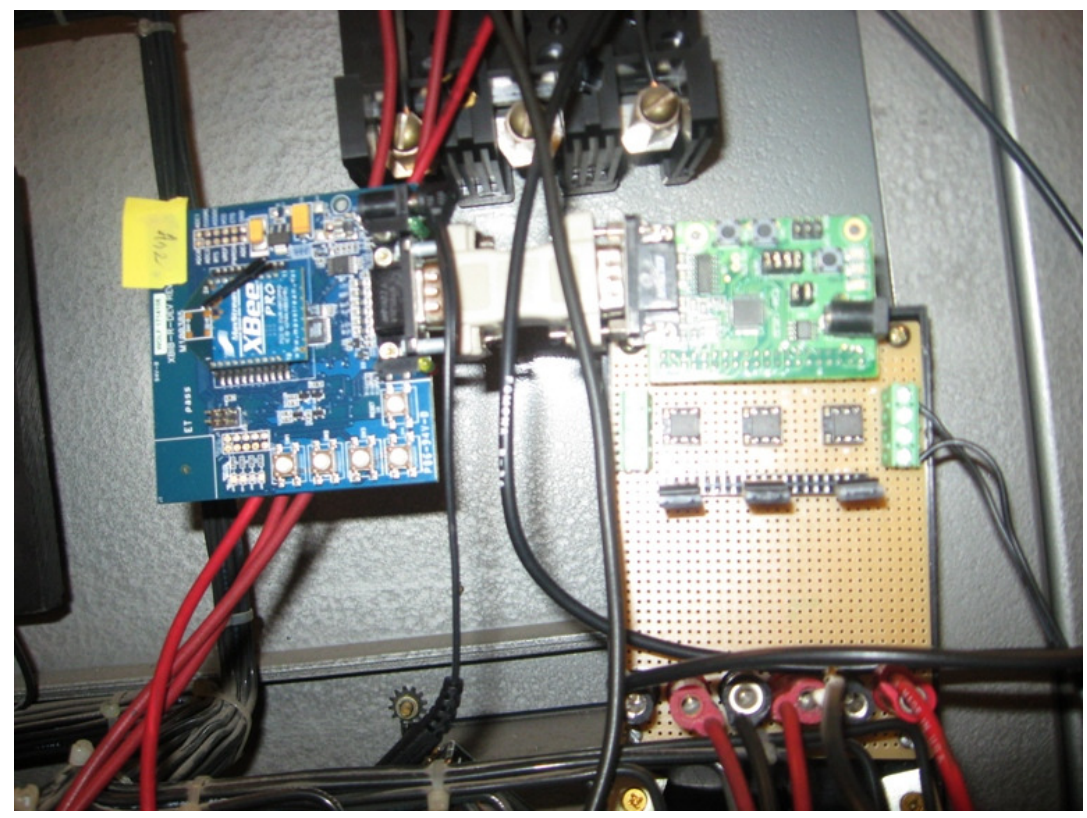

Figure 5.39 Agent prototype on Simulator

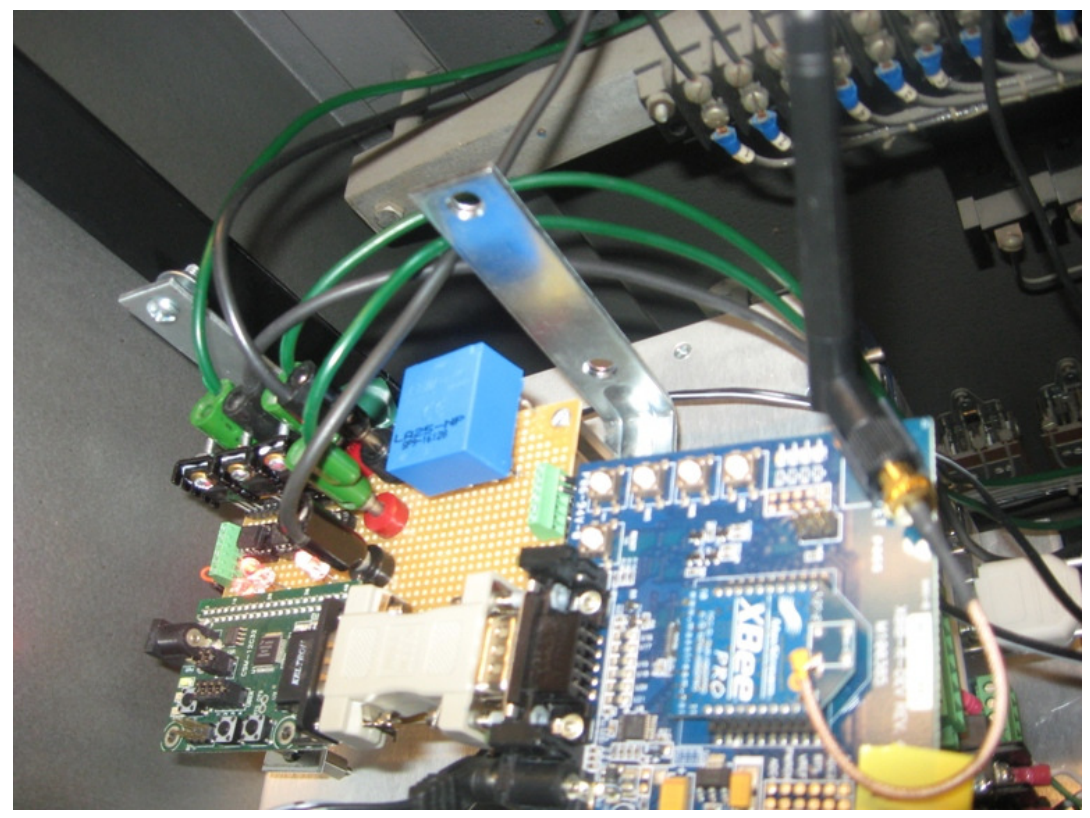

Figure 5.40 Agent prototype on Simulator

For illustration, the one line diagram of the power simulator showing the placement of the agent's hardware depicted in Figure 5.41. The figure also shows the zoning of the power simulator similar to the WVSC layout. 


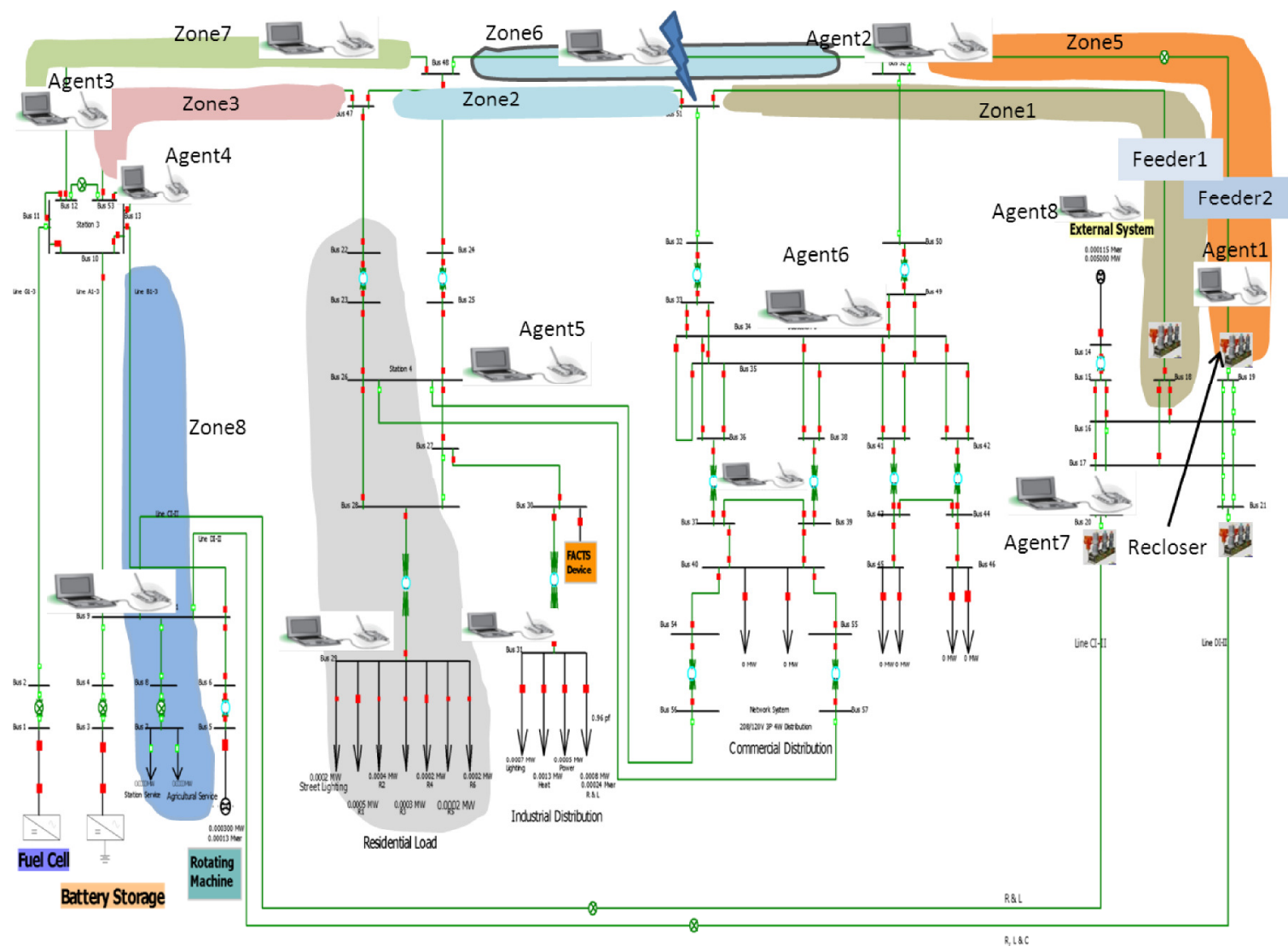

Figure 5.41 Simplified one line diagram of the simulator

\section{Fault detection Isolation and reconfiguration scenario}

In the following, a circuit path similar to the West Virginia Super Circuit one is identified on the power simulator hardware to replicate the same fault scenario performed previously. For illustration, a simplified version of the one line diagram of the power simulator hardware showing the affected portion and the placement of the agent's hardware is depicted in Figure 5.41. The figure also shows the zoning of the power simulator similar to the WVSC layout.

To test the developed Multi Agent System hardware, a three phase permanent fault is applied on Feeder\#2 in ZONE\#6. In the pre-fault state, an unbalanced load is being supplied from one of the main feeders of the system (Feeder\#2) and an unbalanced residential area is supplied through a second feeder (Feeder\#1), see Figure 5.41.

At the moment the fault is applied on the feeder, the first protective device, which is the developed electronic recloser, is automatically triggered. The recloser turns on and off 3 times before the permanent lockout is applied since the fault is maintained on the feeder line. In this 
case the recloser closes and opens for a shorter period of time compared to the computer simulation performed earlier to avoid damage to the equipment.

The same flow of information and action is observed in the hardware side that is: tripping of the DG, isolation of the fault, then reconfiguration to restore power to the healthy zones.

As it can be seen from Figure 5.42, the faulted feeder is totally de-energized to meet the requirement set in the WVSC design.

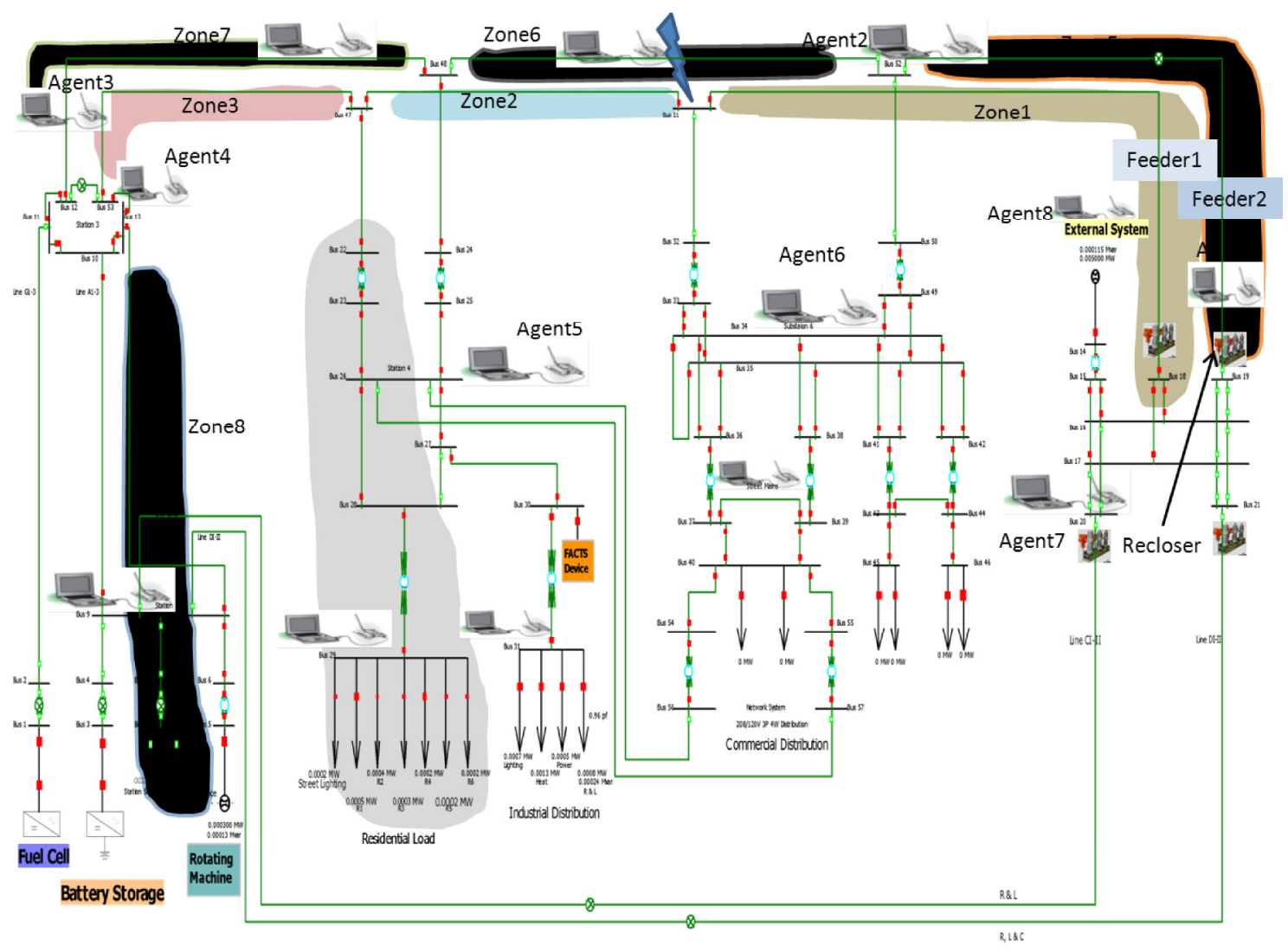

Figure 5.42 Fault on feeder\#2

Once the recloser locks out, the Agent residing at the substation send a permission signal to the zonal agents to start the isolation and reconfiguration. Figure 5.43 below shows the state of the system after reconfiguration and restoration of the circuit. 


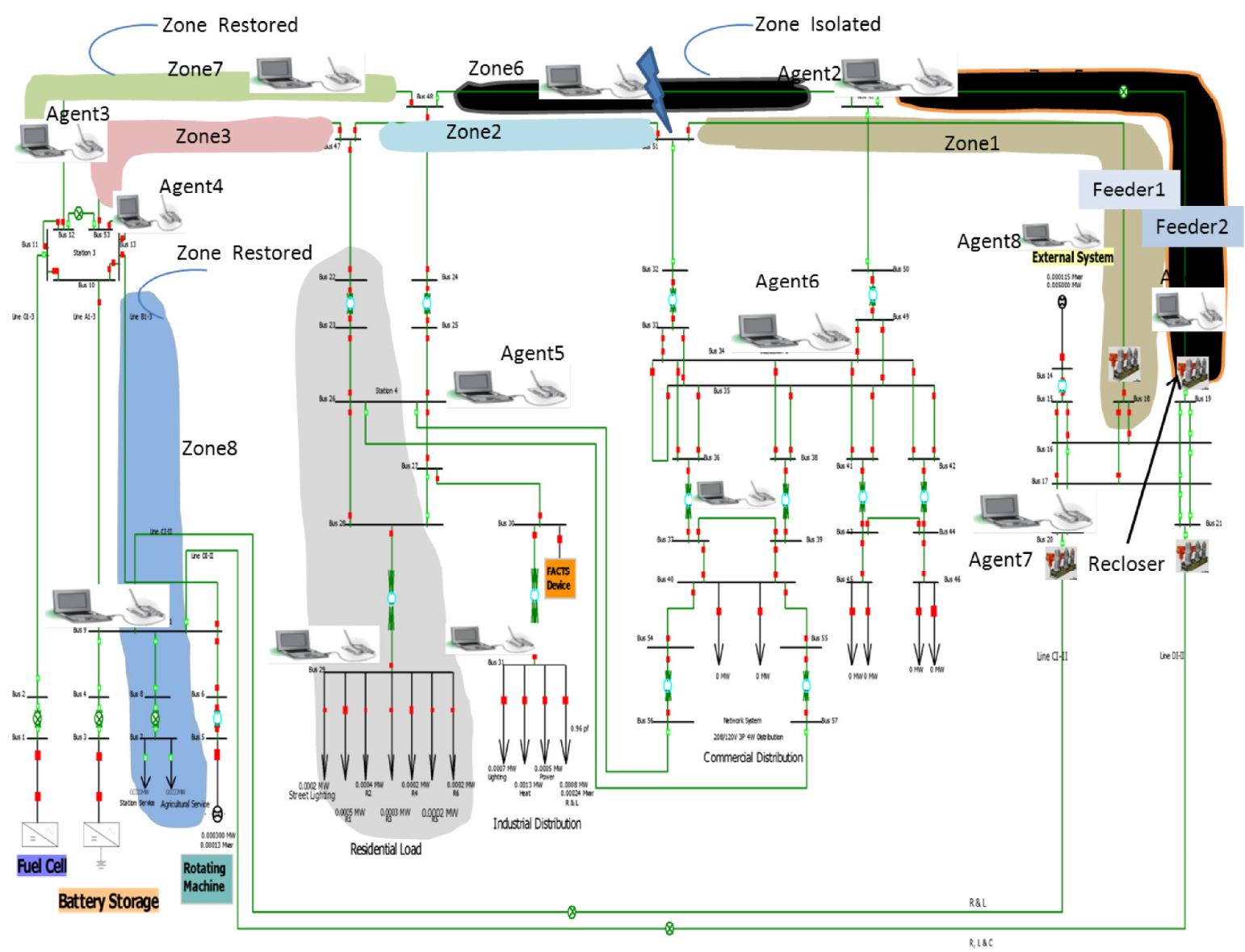

Figure 5.43 Reconfiguration restoration process

In the following we are showing the experimental data of current waveforms collected during the scenario.

Figure 5.44 depicts the current flowing through Feeder\#1. The waveforms show the pre-fault, during fault, and after fault current waveform.

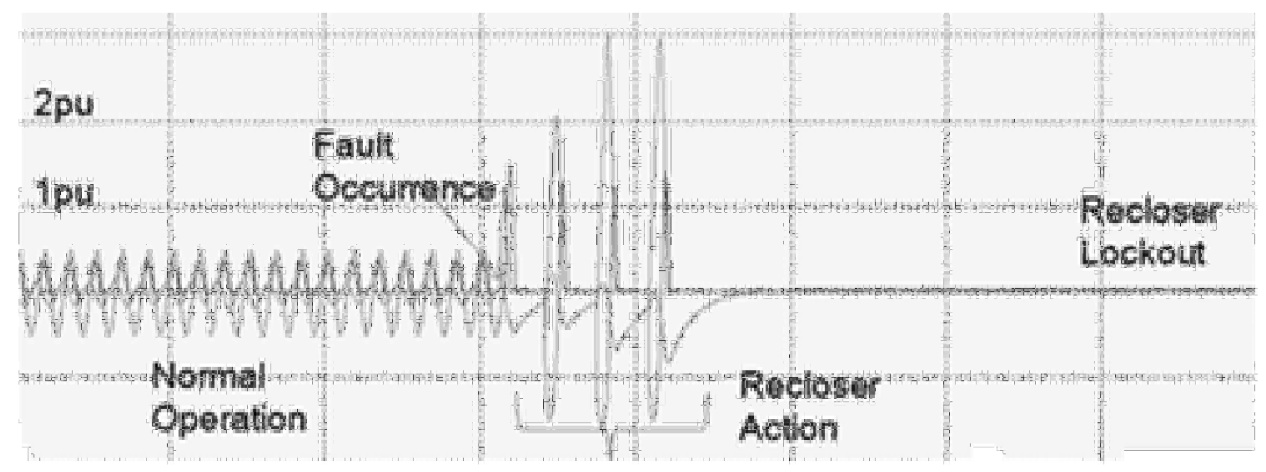

Figure 5.44 Experimental Data of the current on the $1^{\text {st }}$ Feeder 
Figure 5.45 depicts the current waveform measured in zone\#3 of Feeder\#1 (seen at the customer level). From data we can observe that the customer power outage period is about half a second for this particular scenario.

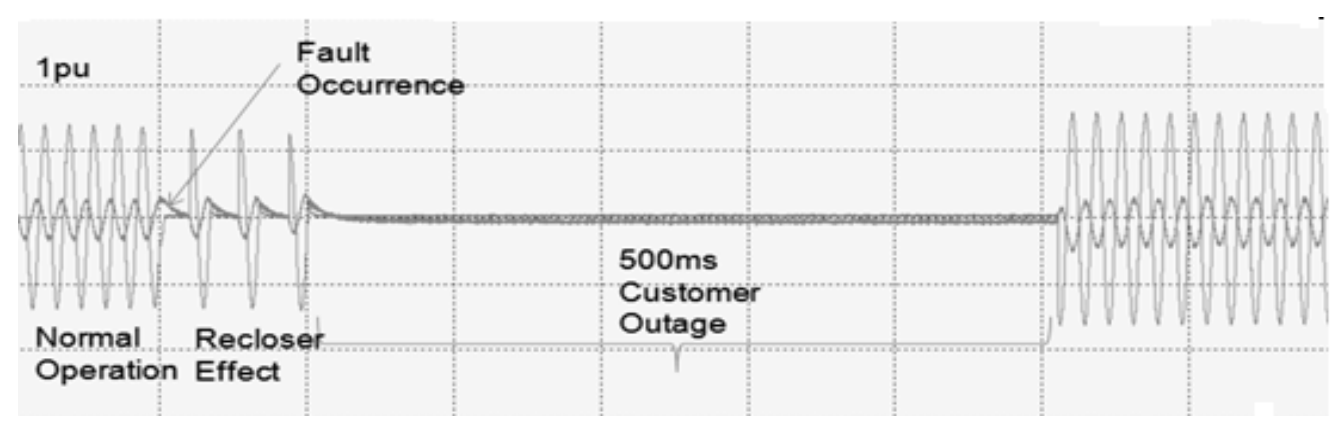

Figure 5.45 Experimental Data of the Current at the load level

Figure 5.46 depicts the current waveform measured in at the Feeder\#2. We can observe that the increase in the current due to restoration of Zone\#3.

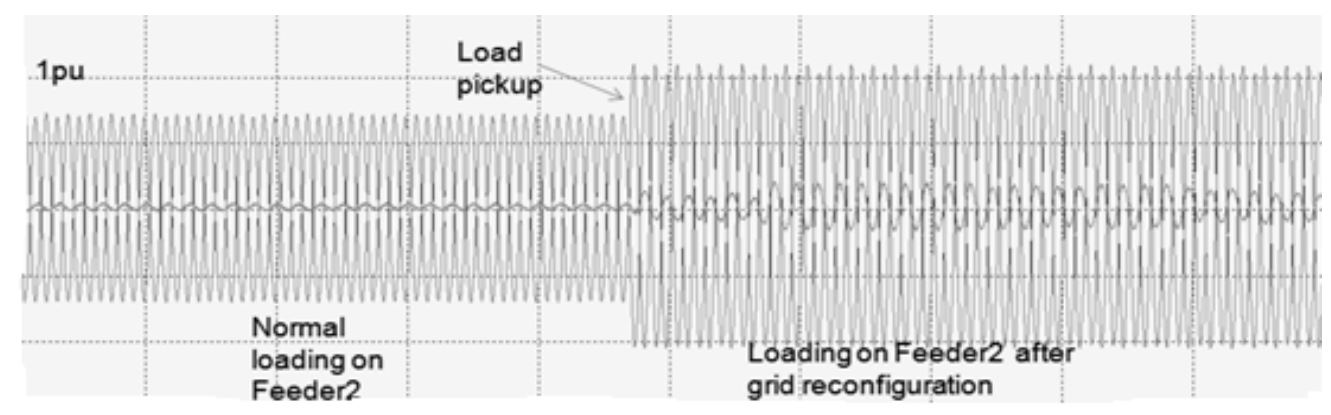

Figure 5.46 Experimental Data of the current on the $2^{\text {nd }}$ feeder

Figure 5.47 shows a time synchronized measurement of a phase current of the faulted feeder at the substation level versus the phase current of the DG. We can observe the fast tripping of the DG by the Agents right after the fault occurred in the system to avoid damages. 


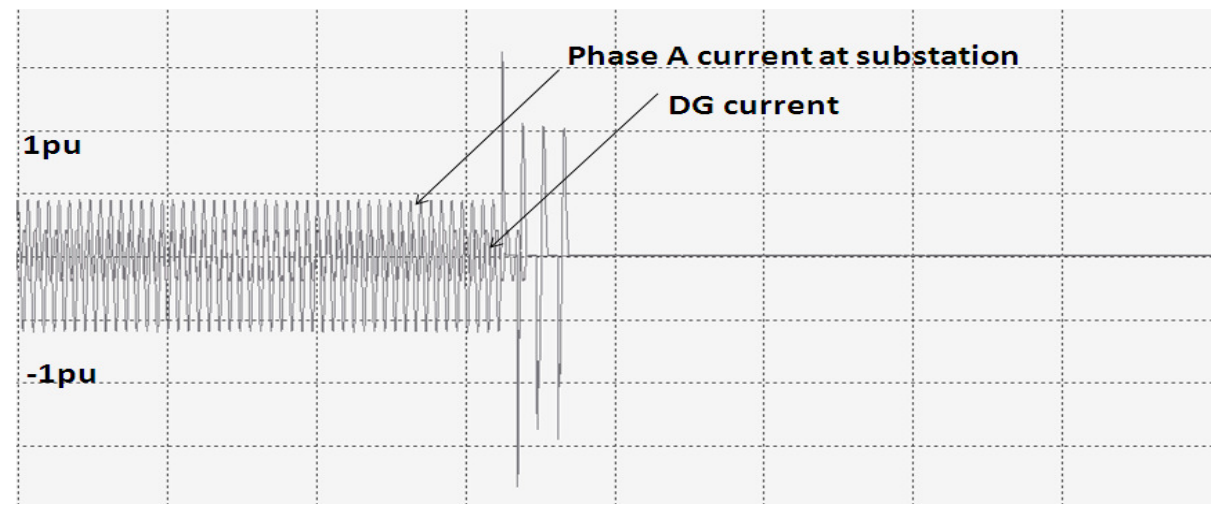

Figure 5.47 Phase current at the substation versus current at the DG

The waveforms depicted in Figure 5.48 show the reconection of the DG by the agents to restore Zone\#8 situated at the end of the faulted Feeder\#2.

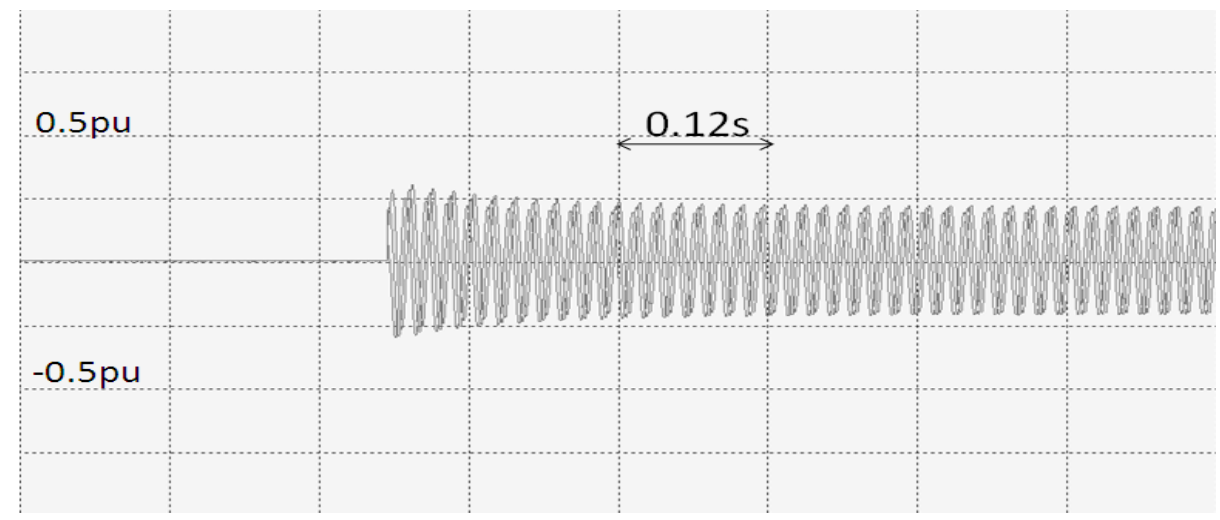

Figure 5.48 Experimental Data of the current waveform at the DG

\subsection{Conclusion}

In this chapter we addressed some aspects on how to design Multi Agents System hardware for smart grid development and integration. First, we have given a brief introduction on smart grid perspective and benefits. Then, we presented the new WVSC project the APERC team is working on. After that we talked about the first phase of the project which consists in developing a small scale smart circuit by modifying and upgrading the existing Analogue Power Simulator to demonstrate the effectiveness of the implemented technologies. Afterwards, we showed how to develop the agent hardware along with the wireless communication platform and the electronic switches. After putting together the different designed pieces, the resulting Multi Agent System is integrated into the Power Simulator. Finally, the multi Agent System is tested for fault 
isolation reconfiguration and restoration problem by simulating a permanent three phase fault on one of the feeder lines. The experimental results show that the technology is very effective and promising. 


\section{Chapter 6}

\section{CONCLUSION AND FUTURE WORK}

In this Chapter a brief conclusion about the work presented in the previous chapters is given along with the work that still needs to be performed in the future.

\subsection{Conclusion}

The work presented in the previous chapters is divided into three sections. In the first section we addressed the problem of Automatic Control Generation design. In this section the Clonal Selection theory is successfully applied as an optimization technique to obtain decentralized control gains that minimize a performance index based on Area Control Errors. Secondly, the Immune Network theory is used to design adaptive controllers in order to diminish the excess maneuvering of the units and help the control areas comply with NERC's standards set to insure good quality of service and equitable mutual assistance by the interconnected energy balancing areas. The results show the effectiveness of the immune based controllers over conventional controllers in the case of Two Area system.

The second section of this work addressed the design and deployment of a multi agent system on both terrestrial and shipboard power system for fault detection, isolation, reconfiguration, and load shedding purposes using novel approach based on the Immune system Multi-Agent system. The Immune System is viewed as a highly organized and distributed Multi-Cell System that strives to heal the body by working together and communicating to get rid of the pathogens. In 
this work both simulation and hardware design and deployment of the MAS are addressed. The MAS design is based on the immune system algorithm in which the agents are trained and embodied with power system information. The MAS adapts to the change in the topology by communicating local information. The agents in this work are programmed in the Freescale MC9S12C32 microcontrollers and integrated in the testbed hardware. The simulation and experimental results show that the agents are capable of performing very fast reconfiguration and load shedding schemes.

In the third section of this work we addressed the design of Multi-Agent System hardware for smart grid development and integration. The Multi-Agent System is identified by the US department of energy as one of the advanced control technologies of the future grid. One of the challenges identified in this field is the inability to predict the outcome of applying such technology on the entire system due to scarcity of experimental tests. It's for this reason that a smart grid power system prototype needed to be developed. In this work, first we have given a brief introduction on smart grid perspective and benefits. Then, we presented the new WVSC project the APERC team is working on. After that we talked about the first phase of the project which consists in developing a small scale smart circuit by modifying and upgrading the existing Analogue Power Simulator to demonstrate the effectiveness of the implemented technologies. Afterwards, we showed how to develop the agent hardware along with the wireless communication platform and the electronic switches. After putting together the different designed pieces, the resulting Multi Agent System is integrated into the Power Simulator. Finally, the multi Agent System is tested for fault isolation reconfiguration and restoration problem by simulating a permanent three phase fault on one of the feeder lines that includes a distributed generator (DG). The experimental results show that the multi agent system hardware developed performed effectively and in a timely manner which confirms that this technology is very promising and a very good candidate for smart grid control applications. 


\subsection{Future Work}

The very next steps of work that are intended to expand are:

- WVSC Modeling: a more detailed modeling of the test circuit in an RTDS station would be a good addition to validate the simulation results and uncover new problems related to communication delays and measurements synchronization.

- Hardware development: The power simulator can be a powerful tool for testing and validating. One of the most important additions is digitalizing all the equipment and integrating smart grid technologies such as storage systems and wind turbine. After analyzing the existing power simulator circuit diagram, we can see that it does not offer much flexibility in terms of reconfiguration and fault analysis. The addition of loads on the feeder lines would allow the creation of multiple zones similar to the actual distribution systems and would offer a much better test system to validate any power management algorithms.

- Fault detection: in the work presented here, the fault detection part of the algorithm is based on current measurements only. The addition of voltage measurements can increase the robustness of the algorithm and validate the fault location.

- Software interfacing: dynamic databases are used to interface the power system model developed in MATLAB with the Multi Agent System developed in JADE platform. The lack of synchronization between the two software systems sometimes causes the Jade Platform to crash. A more sophisticated and smooth interface would allow for an easier integration and deployment of the Multi Agent System on the power system model. 


\section{PUBLICATIONS AND AWARDS}

- Rabie Belkacemi, Ali Feliachi, M.A.Choudhry, "Multi-Agent Systems Hardware Development and Deployment for Smart Grid Control Applications," IEEE Transactions on Smart Grid, 2011 (In Review).

- Rabie Belkacemi, Ali Feliachi, M.A.Choudhary, "Multi-Agent Systems Hardware Development and Deployment for Smart Grid Control Applications," Proc. of the IEEE Power Engineering Society General Meeting, Michigan, 2011 (accepted).

- Ali Feliachi, Rabie Belkacemi, “Intelligent Multi-Agent System for Smart Grid Power Management," Smart Power Grids, Edited by Ali Keyhani, Muhammad Marwali, Publisher SPRINGER-VERLAG, 2011(invited book chapter).

- Rabie Belkacemi, Ali Feliachi,"Design and Deployment of a Multi-Agent System on a Hardware Prototype," Proc. of the IEEE Power Engineering Society General Meeting, July 25-29, 2010, Minneapolis, Minnesota.

- Rabie Belkacemi, Ali Feliachi, "Multi-Agent Design for Power Distribution System Reconfiguration Based on the Artificial Immune System Algorithm," Proc. of the IEEE Circuits and Systems Society ISCAS 2010, May 30-June 2, 2010, France.

- Rabie Belkacemi, Ali Feliachi,"A Decentralized Immune Based Automatic Generation Control Design in Compliance with NERCs Standards," Proc. of the IEEE Power Engineering Society PSCE09, March 15-18, 2009, Seattle, WA.

Rabie Belkacemi, Ali Feliachi, "An Immune System Approach for Power System Automation and Self-Healing," Proc. of the IEEE Power Engineering Society IEEE PSCE09, March 15-18, 2009, Seattle, WA. 
- Rabie Belkacemi, Ali Feliachi, "Immune Based Design of a Load Frequency Controller in Power Systems," Proc. International Conference on Electrical Engineering Design and Technologies: ICEEDT, Nov. 4-6, 2007 Tunis, Tunisia.

- R. Belkacemi, A. Feliachi, "Immune Based Design of a Load Frequency Controller in Power Systems," Journal of Electrical Systems, Vol.4, Issue 2, pp. 1-12, June 2008

- Rabie Belkacemi, Ali Feliachi,"Decentralized Immune Based Design of Load Frequency Controller in Power Systems," Proc. of the STaR Symposium, Sept. 17-18, 2007, Morgantown, WV.

- Ali Feliachi, Rabie Belkacemi, "Immune Based Control Design for Power systems," Intelligent Techniques for Power System Transmission, Edited by G.Venayagamoorthy, N.Hingorani and R.G.Harley, Wiley Inc. 2008. (Invited Chapter).

- IEEE Power Engineering Society Award: 3rd prize winner award for the best poster at the IEEE Power Engineering Society General Meeting, Pittsburgh, July 2008 


\section{REFERENCES}

[1] E. Hirst, and B. Kirby, "Ancillary Service Details: Regulation, Load Following, and Generator Response," Oak Ridge National Laboratory, Oak Ridge, TN, Tech. Rep. ORNL/CON433, Sep. 1996.

[2] North American Electric Reliability Council (NERC), "Performance Standard Training Document,"in Operating Manual, pp. ps1-20, Nov. 1996

[3] Electric Power Research Institute (EPRI), "Interconnected Power System Dynamic Tutorial,” EPRI TR-107726 1915-16 Final Report, Palo Alto, CA, March 1997

[4 ] N. Jaleeli, and L. S. VanSlyck, "NERC's New Control Performance Standards," IEEE Transactions on Power Systems, Vol. 14, No. 3, pp. 1092-1099, August 1999

[5] NETL report "Advanced control methods", Appendix B4, A Systems View of the Modern Grid, 2010

[6] K. P. Schneider et all, "Modern Grid Initiative Distribution Taxonomy Final Report," Pacific Northwest National Laboratory, November 2008

[7] Y. L. Abdel-Magid, and M. M. Dawoud, "Genetic Algorithms Applications in Load Frequency Control," The First International Conference on Genetic Algorithms in Engineering Systems: Innovations and Applications, pp. 207-213, 1995

[8] W. C. Schultz, and V. C. Rideout, "Control System Performance Measures: Past Present and Future," IEEE Transactions on Automatic Control, AC-6, 22 pp. 22-35, 1961

[9] V. Donde, M. A. Pai, and I. A. Hiskens, "Simulation and Optimization in an AGC System after Deregulation," IEEE Transactions on Power Systems, Vol. 16, no. 3, pp. 481-489, August 2001 
Chapter 6: Conclusion \& Future Work

[10] A. Feliachi, "On Load Frequency Control in a Deregulated Environment," Proceedings of the 1996 IEEE International Conference on Control Applications, pp. 437-441, 1996

[11] M. H. Rahi, and A. Feliachi, "Hळ Robust Decentralized Controller for Nonlinear Power Systems," Proceedings of the 30th Southeastern Symposium on Syspm Theory, pp. 268-270, March 1998

[12] A. Feliachi, "Reduced $H \infty$ load frequency controller in a deregulated electric power system environment," Proceedings of the 36th IEEE Conference on Decision and Control, Vol. 4, pp. 3100-3101, 1997

[13] M. Aldeen, and J. F. Marsh, "Decentralized Proportional-Plus-Integral Design Method for Interconnected Power Systems," IEE Proceedings-C, Vol. 138, No. 4, pp. 263-274, July 1991.

[14] H. Trinh, and M. Aldeen, "Decentralized Load-Frequency Control of Interconnected Power Systems," IEE International Conference on Advances in Power System Control, Operation and Management, Vol. 2, pp. 815-820, 1991.

[15] M. Aldeen, "Interaction Modeling Approach to Distributed Control with Application to Power Systems," International Journal Control, Vol. 53, pp. 1035-1054, 1991

[16] S. S. Stankovic, X. Chen, M. R. Matausek, and D. D. Siljak, "Stochastic Inclusion Principle Applied to Decentralized Automatic Generation Control," International Journal Control, Vol. 72, No. 3, pp. 276-288, 1999

[17] K. Y. Lim, Y. Wang, and R. Zhou, "Robust Decentralized Load-Frequency Control of Multi-Area Power Systems," IEE Proceedings-Gener. Transm. Distrib. Vol. 143, No. 5, pp. 377386, September 1996

[18] K. Y. Lim, Y. Wang, G. Gua, and R. Zhou, “A New Decentralized Robust Controller Design for Multi-Area Load-Frequency Control via in Complete State Feedback," Optimal Control Applications \& Methods, Vol. 19, pp. 345-361, 1998 
Chapter 6: Conclusion \& Future Work

[19] Y. Hsu, and C. Cheng, "Load Frequency Control Using Fuzzy Logic," International Conference on High Technology in the Power Industry, pp. 32-38, 1991

[20] M. Djukanovic, "Two-Area Load Frequency Control with Neural Nets," Proceedings of the North American Power Symposium, pp. 161-169, 1993

[21] J. Talaq, and F. Al-Basri, "Adaptive Fuzzy Gain Scheduling for Load Frequency Control,” IEEE Transactions on Power Systems, Vol. 14, No. 1, pp. 145-150, February 1999

[22] G. B. Sheble, Computational Auction Mechanisms for Restructured Power Industry Operation, Kluwer Academic Publishers, Norwell, MA, 1999, ISBN 079238475X.

[23] N. Jaleeli, and L. S. VanSlyck, "NERC's New Control Performance Standards," IEEE

Transactions on Power Systems, Vol. 14, No. 3, pp. 1092-1099, August 1999

[24] N. Jaleeli, and L. S. VanSlyck, "Tie-Line Bias Priortized Energy Control," IEEE Transactions on Power Systems, Vol. 10, No. 1, pp. 51-59, February 1995

[25] M. Yao, R. R. Shoults, and R. Kelm, “AGC Logic Based on NERC's New Control PerformanceStandard and Disturbance Control Standard," IEEE Transactions on Power Systems, Vol. 15, No. 2, pp. 852-857, May 2000

[26] T. Nagata, H. Sasaki and R. Yokoyama, "Power System Restoration by Joint Usage of Expert System and Mathematical Programming Approach," IEEE Transactions on Power Systems, Vol. 10, No. 3, pp 1473 - 1479, August 1995

[27] R. Belkacemi, A. Feliachi, "An Immune System Approach for Power System Automation and Self-Healing," Proc. of the IEEE Power Engineering Society IEEE PSCE09, March 15-18, 2009, Seattle, WA

[28] T. Nagata, N. Iba, Y. Tao and H. Sasaki, “A New Power System Restoration Framework by means of Multi-agent Approach," IEEE/PES Transmission and Distribution Conference and Exhibition, Vol. 3, pp. 1695 - 1700, October 2002 
Chapter 6: Conclusion \& Future Work

[29] T. Nagata and H. Sasaki, “A Multi-agent Approach to Power System Restoration,” IEEE Transactions on Power System, Vol. 17, No. 2, pp 457 - 462, May 2002

[30] T. Nagata, Y. Tao and H. Fujita, “An Autonomous Agent for Power System Restoration," IEEE Power Engineering Society General Meeting, Vol. 1, pp 1069 - 1074, June 2004

[31] T. Nagata, Y. Tahara and H. Fujita, "An Agent Approach to Bulk Power System Restoration," IEEE Power Engineering Society General Meeting, Vol. 1, pp 599 - 604, June 2005

[32] T. Nagata, Y. Tao, Y. Tahara and T. Aoyama, "Development of Bulk Power System Restoration Simulator by means of Multi-agent Approach," The 47th IEEE International Midwest Symposium on Circuits and Systems, Vol. 2, pp 337 - 340, July 2004

[33] D. Liu, Y. Chen, G. Shen and Y. Fan, "Multi-agent based Approach for Modeling and Simulation of Bulk Power System Restoration," IEEE/PES Transmission and Distribution Conference and Exhibition, pp 1- 6, 2005

[34] J. M. Solanki, N. N. Schulz and W. Gao, "Reconfiguration for Restoration of Power Systems using Multi-Agent System," Proceedings of the 37th Annual North American Power Symposium, pp 390 - 395, October 2005

[35] J. M. Solanki, S. Khushalani and N. N. Schulz, "A Multi-Agent Solution to Distribution Systems Restoration," IEEE Transactions on Power Systems, Vol. 22, No. 3, pp 1026 - 1034, August 2007

[36] J. G. Gomez-Gualdron and M. Velez-Reyes, "Simulating a Multi-Agent based SelfReconfigurable Electric Power Distribution System," IEEE COMPEL Workshop, Rensselaer Polytechnic Institute, NY, July 2006

[37] J. G. Gomez-Gualdron, M. Velez-Reyes and L. J. Collazo, "Self-Reconfigurable Electric Power Distribution System using Multi-Agent Systems,” Electric Ship Technologies Symposium, pp 180 - 187, May 2007 
Chapter 6: Conclusion \& Future Work

[38] K. Huang, S. Srivastava and D. Cartes, "Decentralized Reconfiguration for Power Systems using Multi Agent System,” 1st Annual IEEE Systems Conference, pp 1 - 6, April 2007

[39] S. Chouhan, Hui Wan, H.J. Lai, A. Feliachi, M.A. Choudhry, "Intelligent reconfiguration of smart distribution network using multi-agent technology ," Power \& Energy Society General Meeting, 2009

[40] K. Nareshkumar, M.A. Choudhry, J.Lai, A. Feliachi, "Application of multi-agents for fault detection and reconfiguration of power distribution systems," IEEE Power \& Energy Society General Meeting, 2009, PES '09.

[41] Goldsby RA, Kindt TJ, and Osborne BA. Kuby Immunology. W.H.Freeman and Company; 2000

[42] Marrack P, Kappler JW. How the immune system recognizes the body. Scientific American pp. 49-55; 1993

[43] Burnet F, The clonal selection theory of acquired immunity. Cambridge University Press; 1959

[44] Sprent J. T and B memory cells. Cell, vol. 76, pp. 315-322, January 28; 1994

[45] Jerne NK. Towards a Network Theory of the Immune System. Annals of Immunology, (Inst.Pasteur) 125C, pp. 373-389; 1974

[46] de Castro LN, Timmis J. Artificial Immune Systems: A New Computational Intelligence Approach. Springer-Verlag; 2002

[47] D. Dasgupta, "Artificial Immune Systems and Their Applications," Berlin: Springer; 1998

[48] Feliachi A. On the Control of Re-Structured Electric Power Systems. International Journal of Control, Automation, and Systems, vol. 3, no. 2 (special edition), pp. 363-375 ; June 2005 
Chapter 6: Conclusion \& Future Work

[49] Belkacemi R, Feliachi A, "Immune Based Design of a Load Frequency Controller in Power System", Proceedings, International Conference on Electrical Engineering Design and Technologies: ICEEDT, Hammamet, Tunisia, Nov 4-6, 2007

[50] G. Weiss, "Multiagent Systems: A Modern Approach to Distributed Artificial Intelligence," The MIT Press, 2000

[51] M. Wooldridge, N. R. Jennings, "Intelligent agents: Theory and Practice," The Knowledge Engineering Review, Vol. 10, Issue 2, pp 115 - 152, 1995

[52] S. D. Pekarek, J. Tichenor, S. D. Sudhoff, J. D. Sauer, D. E. Delisle, and E. J. Zivi, "Overview of a naval combat survivability program," Proceedings of the 13th International Ship Control Systems Symposium, Orlando, Florida, 2003

[53] S9086-KY-STM-010/CH-320R2, Naval Ships Technical Manual Chapter 320, Electric Power Distribution Systems, Direction of Commander, Naval Sea Systems Command, Apr 1998.

[54] H.Inan, "West Virginia Super Circuit Project Preliminary Design Document", Version 2.0, October 11, 2010

H.Inan,

"MGMS_FLIR_UseCase",

November

3 , 2010 
References

John H.

ou=Acquisitions Department,

Hagen 